\title{
Um ensaio em teoria dos jogos
}

\author{
Edgard Almeida Pimentel
}

\author{
DisSERTAÇÃO APRESENTADA \\ $\mathrm{AO}$ \\ Instituto De MatemáticA E Estatística \\ DA \\ Universidade de SÃo Paulo \\ PARA \\ OBTENÇÃO DO TÍTULO \\ $\mathrm{DE}$ \\ Mestre em CiênCias
}

\author{
Programa: Matemática Aplicada \\ Orientador: Prof. Dr. Pedro Aladar Tonelli
}

Durante o desenvolvimento deste trabalho o autor recebeu auxílio financeiro da CAPES e do CNPq (Brasil) e da FCT-IST-UTL e do CAMGSD-IST-UTL (Portugal). 


\section{Um ensaio em teoria dos jogos}

Este exemplar corresponde à redação

final da dissertação devidamente corrigida

e defendida por Edgard Almeida Pimentel

e aprovada pela Comissão Julgadora.

Banca Examinadora:

- Prof. Dr. Pedro Aladar Tonelli (orientador) - IME-USP.

- Prof.a. Dr.a. Helena J. Nussenzveig Lopes - IMECC-UNICAMP.

- Prof. Dr. Milton da Costa Lopes Filho - IMECC-UNICAMP. 
"Choo choo ch'boogie"

Louis Jordan

"Digo: o real não está na saída nem na chegada: ele se dispõe para a gente é no meio da travessia" João Guimarães Rosa

"Ich suche immerfort etwas Nicht-Mitteilbares mitzuteilen, etwas Unerklärbares zu erklären"

Franz Kafka 


\section{Agradecimentos}

Este trabalho encerra uma etapa de transição. Como em toda transição, encontrei diversas situações esperadas e inesperadas. Alcançar a elaboração intelectual necessária para transitar do raciocínio concreto de uma ciência com 300 anos para o raciocínio abstrato da Senhora das Ciências, com mais de 3000 anos de idade, é como um caminho em que o peregrino deve trazer no chapéu as conchas que lhe identificam o propósito e atenuam a caminhada. As conchas que carreguei no meu chapéu, até aqui, são mencionadas nestes agradecimentos.

Agradeço aos meus pais, por estarem sempre dispostos. Por influenciarem os meus valores e marcarem o meu caráter. Sobretudo por me oferecerem alguns de meus maiores tesouros: o senso crítico e a independência. Agradeço ainda a acurada revisão de papai sobre o texto. À minha tia Gilda, agradeço pelo primeiro, segundo, ..., n-ésimo livro. E por completar minha coleção de obras do Franz Kafka antes dos 12 anos de idade. Ao meu avô que, além do Democrático Futebol Clube e do Cacau, ajudou a fundar e jogou sempre no meu time.

Agradeço ao meu orientador Pedro Aladar Tonelli. Por me preparar para a carreira em Matemática, por me ajudar com coisas que eu não entendia e por me fascinar com a importância da simplicidade nos argumentos. Aprendi com o Pedro que o ato de fala mais importante na Matemática é o perlocucional: o efeito real e o alcance do que é dito e seu poder de ser inteligível superam as investidas parnasianas tão comuns na profissão. Pela amizade, pela oportunidade de aprender e pela presença nesta etapa da minha vida. Registro que se um dia eu escrever uma linha que seja de relevante em Matemática divido o mérito com o Pedro.

Agradeço aos membros da banca, professora Helena Nussenzveig Lopes e professor Milton da Costa Lopes pela oportunidade de enriquecer meu trabalho. Seus comentários - que demonstram a Matemática praticada por ambos - além de me ajudarem no entendimento de meu tópico, reforçam minha segurança quanto ao meu interesse pelo tema. Especialmente, agradeço ao professor Milton pela atenção que sempre dispensou quando discutimos os rumos de meu doutoramento.

Agradeço aos membros da banca de meu exame de qualificação, professores Antonio Luiz Pereira e Sergio Muniz Oliva Filho. Apesar de ter me preparado para a prova, acredito ter aprendido mais durante a argüição do que antes dela.

Agradeço aos professores do IME com os quais tive a oportunidade de trabalhar na posição de aluno monitor ou estagiário PAE, nominalmente, Sonia Regina Leite Garcia e Frank Michael Forger. Ambos geraram um ambiente em que meu envolvimento com as disciplinas pôde ser o mais enriquecedor possível.

Agradeço à professora Mary Lilian Lourenço por me aceitar nos seus cursos e ter se tornado uma 
pessoa querida dentro do Instituto. Por me falar sempre coisas boas, eu agradeço. Ao professor Gláucio Terra, por me aceitar em seu curso e, sobretudo, ter sido uma das pessoas mais atenciosas que eu encontrei. Ao professor Antonio de Pádua. Pelas conversas, pela amizade e pela coragem de discutir as coisas comigo.

Agradeço aos funcionários do IME-USP. Especialmente aos colegas da CPG: Pinho, Marilucia Otama e Alessandra Bernardo. Pelo empenho em tornar coisas importantes possíveis. Em outros termos, por quebrarem galhos de fato importantes.

Em Lisboa, tive a felicidade de interagir com o professor Diogo Aguiar Gomes, a quem sou grato pela atenção e pelo apoio fundamental. A confiança que o Diogo depositou em mim foi decisiva para que eu pudesse me envolver com o tema de meu interesse no melhor ambiente possível. Agradeço ao professor Carlos Rocha que, através do CAMGSD, pôde tornar minha chegada em Portugal mais suave. Agradeço ainda aos professores Pedro Girão, João Santos e Lina Oliveira, pelos cursos que assisti e à professora Sofia Naique, pelo apoio irrestrito no Instituto.

Agradeço a Daniel Valesin, que me ajudou com os primeiros passos nas coisas da Matemática.

Aos meus amigos, Raul Antonio Cristóvão dos Santos, Raul Cristóvão dos Santos e Leandro Almeida. Por terem sido meus amigos quando eu falhei, e por tomarem minhas desculpas esfarrapadas por verdades entre amigos. Aprendi muitas coisas observando estas pessoas e o afeto que nutro por elas serve de parâmetro para que eu entenda melhor meu lugar no mundo. E de cada encontro que tenho com cada um deles saio diferente.

Agradeço à Juliana, por ser a melhor companhia possível, para qualquer coisa. Por abrir portas que eu não sabia que existiam e por me ensinar a ser menos pior. Por construir coisas, por cuidar de mim e por aceitar ser o centro do mundo que, segundo ela, só existe na minha cabeça. Agradeço também pela oportunidade do convívio com pessoas queridas como o seu Godo, a dona Jane e o Derson Luís Inácio (ou Antonio). 


\section{Resumo}

Esta dissertação aborda a teoria dos jogos diferenciais em sua estreita relação com a teoria das equações de Hamilton-Jacobi (HJ). Inicialmente, uma revisão da noção de solução em teoria dos jogos é empreendida. Discutem-se nesta ocasião as idéias de equilíbrio de Nash e alguns de seus refinamentos. Em seguida, tem lugar uma introdução à teoria dos jogos diferenciais, onde

noções de solução como a função de valor de Isaacs e de Friedman são discutidas. É nesta altura do trabalho que fica evidente a conexão entre este conceito de solução e a teoria das equações de Hamilton-Jacobi. Por ocasião desta conexão, é explorada a noção de solução clássica e é exposta uma demonstração do fato de que se um jogo diferencial possuir uma função de valor pelo menos continuamente diferenciável, esta será uma solução da equação de Hamilton-Jacobi associada ao jogo. Este resultado faz uso do princípio da programação dinâmica, devido a Bellman, e cuja demonstração está presente no texto. No entanto, quando a função de valor do jogo é apenas contínua, então embora esta não seja uma solução clássica da equação HJ associada a jogo, vemos que ela será uma solução viscosa, ou solução no sentido da viscosidade - e a esta altura são discutidos os elementos e propriedades desta classe de soluções, um teorema de existência e unicidade e alguns exemplos. Por fim, retomamos o estudo dos jogos diferenciais à luz das soluções viscosas da equação de Hamilton-Jacobi e, assim, expomos uma demonstração de existência da função de valor e do princípio da programação dinâmica a partir das noções da viscosidade.

Palavras-chave: Equilíbrio de Nash e refinamentos, jogos diferenciais e função de valor, HamiltonJacobi e soluções viscosas. 


\section{Abstract}

This dissertation aims to address the topic of Differential Game Theory in its connection with the Hamilton-Jacobi (HJ) equations framework. Firstly we introduce the idea of solution for a game, through the discussion of Nash equilibria and its refinements. Secondly, the solution concept is then translated to the context of Differential Games and the idea of value function is introduced in its Isaacs's as well as Friedman's version. As the value function is discussed, its relationship with the Hamilton-Jacobi equations theory becomes self-evident. Due to such relation, we investigate the HJ equation from two distinct points of view. First of all, we discuss a statement according to which if a differential game has a continuously differentiable value function, then such function is a classical solution of the HJ equation associated to the game. This result strongly relies on Bellman's Dynamic Programming Principle - and this is the reason why we devote an entire chapter to this theme. Furthermore, HJ is still at our sight from the PDE point of view. Our motivation is simple: under some lack of regularity - a value function which is continuous, but not continuously differentiable - a game may still have a value function represented as a solution of the associated HJ equation. In this case such a solution will be called a solution in the viscosity sense. We then discuss the properties of viscosity solutions as well as provide an existence and uniqueness theorem. Finally we turn our attention back to the theory of games and - through the notion of viscosity

- establish the existence and uniqueness of value functions for a differential game within viscosity solution theory.

Keywords: Game Theory, Nash equilibria and its refinements, Differential game theory and value functions, Hamilton-Jacobi equations and viscosity solutions. 


\section{Sumário}

Lista de Abreviaturas $\quad$ ix

Lista de Figuras

Lista de Tabelas $\quad$ xiii

1 Introdução $\quad 1$

1.1 Considerações preliminares . . . . . . . . . . . . . . . 1

1.2 Organização do trabalho . . . . . . . . . . . . . . . . . . 2

2 Noções elementares de teoria dos jogos $\quad 3$

2.1 Principais modelos de jogos . . . . . . . . . . . . . . . . . 4

2.1 .1 Forma extensiva . . . . . . . . . . . . . . . . . . 4

2.1.2 Forma normal ou estratégica . . . . . . . . . . . . . . . 8

2.1.3 Forma Bayesiana . . . . . . . . . . . . . . . . . . . . 11

2.1.4 Representações multi-agente e tipo-agente . . . . . . . . . . . . . . . . . 13

2.2 Soluções do jogo: o equilíbrio de Nash . . . . . . . . . . . . . . . . . . . 15

2.2.1 Estratégias dominadas e o jogo residual . . . . . . . . . . . . . 16

2.2.2 O Equilíbrio de Nash: idéias iniciais, existência e não-unicidade . . . . . . . . 18

2.3 O equilíbrio de Nash e a forma extensiva . . . . . . . . . . . . . . . . . . 24

2.4 A forma extensiva e a racionalidade sequencial . . . . . . . . . . . . . 26

2.5 A forma normal e o equilíbrio perfeito . . . . . . . . . . . . . . . . . 32

3 Teoria dos Jogos Diferenciais $\quad 37$

3.1 Exemplos introdutórios . . . . . . . . . . . . . . . . 37

3.2 Definições e resultados preliminares . . . . . . . . . . . . . . . . . . 39

3.3 Existência da função de valor de Isaacs . . . . . . . . . . . . . . . . . . . . . . . 44

3.4 A função de valor de Friedman . . . . . . . . . . . . . . . . . . . . . . 47

3.5 A Solução do Jogo Diferencial e as Equações de HJ . . . . . . . . . . . . . . . . . . 51

4 O Princípio da Programação Dinâmica 53

4.1 Exemplos e considerações preliminares . . . . . . . . . . . . . . . 53

4.2 O princípio da programação dinâmica e as equações de HJ . . . . . . . . . . . . . . 54 
5 Soluções Viscosas da Equação de Hamilton-Jacobi $\quad 63$

5.1 Introdução às equações de Hamilton-Jacobi . . . . . . . . . . . . . . . . . . 63

5.2 Primeiras idéias e alguns exemplos . . . . . . . . . . . . . . . 66

5.3 Definições e propriedades . . . . . . . . . . . . . . . . 68

5.4 Um teorema de existência e unicidade . . . . . . . . . . . . . . . . . . 84

5.4 .1 Módulos de continuidade . . . . . . . . . . . . . . . 85

5.4 .2 Um teorema de comparação . . . . . . . . . . . . . . . . . . . . . . . . . . . . . . . . . . . . . . . .

5.4 .3 Um teorema de existência . . . . . . . . . . . . . . . . . . . . 103

6 Revisitando a Teoria dos Jogos Diferenciais 107

6.1 Introdução . . . . . . . . . . . . . . . . . . . . . . . . 107

6.2 Funções de valor e soluções viscosas da equação HJI . . . . . . . . . . . . . . . . 114

6.3 Comentários finais . . . . . . . . . . . . . . . . . . 125

$\begin{array}{ll}\text { A O Ponto Fixo de Kakutani } & 127\end{array}$

A.1 Alguns comentários preliminares . . . . . . . . . . . . . 127

A.2 Enunciado e demonstração . . . . . . . . . . . . . . . . . 131

$\begin{array}{ll}\text { Referências Bibliográficas } & 135\end{array}$

$\begin{array}{ll}\text { Índice Remissivo } & 138\end{array}$ 


\section{Lista de Abreviaturas}

EDO Equação diferencial ordinária (Ordinary differential equation).

EDP Equação diferencial parcial (Partial differential equation).

EN Equilíbrio de Nash (Nash equilibria).

EP Equilíbrio perfeito (Perfect equilibria).

ES Equilíbrio sequencial (Sequential equilibria).

HJ Equação de Hamilton-Jacobi (Hamilton-Jacobi equation).

HJB Equação de Hamilton-Jacobi-Bellman (Hamilton-Jacobi-Bellman equation).

HJI Equação de Hamilton-Jacobi-Isaacs (Hamilton-Jacobi-Isaacs equation).

PCa Problema de Cauchy (Cauchy problem).

PC Princípio da comparação (Comparison principle).

PM Princípio do máximo (Maximum principle).

PPD Princípio da programação dinâmica (Dynamical Programming Principle).

SCI Semi-contínua inferiormente (Lower semi-continuous).

SCS Semi-contínua superiormente (Upper semi-continuous). 


\section{Lista de Figuras}

2.1 Exemplo de um jogo na forma extensiva. . . . . . . . . . . . . . . . 5

2.2 Exemplos de jogos com conjuntos de informação distintos. . . . . . . . . . . . . . . 7

2.3 Representação, forma extensiva. . . . . . . . . . . . . . . . . . . . . . . 14

3.1 Um exemplo de função convexa. . . . . . . . . . . . . . . . . . . . . . . . 42

3.2 Um exemplo de função côncava. . . . . . . . . . . . . . . . . . . . . . . . . . 42

3.3 Gráfico da aplicação $f(x)=\lfloor x\rfloor \ldots \ldots \ldots \ldots \ldots$

3.4 Gráfico da aplicação $f(x)=\lceil x\rceil \ldots \ldots \ldots \ldots \ldots$

4.1 Exemplo - Programação Dinâmica. . . . . . . . . . . . . . . . . 54

4.2 Gráfico de uma função com as propriedades de $l$ para dado $R>0$. . . . . . . . 61

5.1 Problema de deslocamento e exemplo de trajetórias. . . . . . . . . . . . . 64

5.2 Gráfico da função $u(x)=|x| \ldots \ldots \ldots \ldots$. . . . . . . . . . . . 68

5.3 Gráfico da função $u(x)=|x|-x^{2}-1 \ldots \ldots \ldots \ldots \ldots$. . . . . . . . . 71

5.4 Função $\sigma$, comparação de áreas. . . . . . . . . . . . . . . . . . . . . . . . . . . . . . . . .

5.5 Estabilidade do minimizante . . . . . . . . . . . . . . . . . 76

$6.1(x, y) \longmapsto\left|x^{\frac{1}{2}}-y^{\frac{1}{2}}\right|$ em cinza claro e $(x, y) \longmapsto|x-y|^{\frac{1}{2}}$ em cinza escuro. . . . . . 111

A.1 Exemplos de simplex. . . . . . . . . . . . . . . . . . 127

A.2 Tetraedro. . . . . . . . . . . . . . . . . . . . . 128

A.3 Conjunto de pontos colineares. . . . . . . . . . . . . . . . 128

A.4 Simplex padrão em dimensão 2. . . . . . . . . . . . . . . . . . . . 129

A.5 Exemplo de divisão baricêntrica do triângulo . . . . . . . . . . . . . . . . . 129

A.6 Segunda divisão baricêntrica do triângulo. . . . . . . . . . . . . . 130

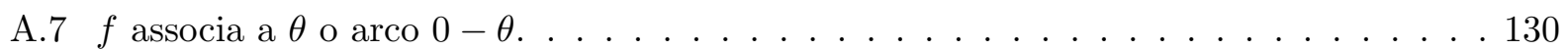

A.8 Gráficos, $\psi_{1}, \psi_{2}$ e $\psi_{3}$, respectivamente. . . . . . . . . . . . . . 134 


\section{Lista de Tabelas}

2.1 Dilema do prisioneiro, jogador $1 . \ldots \ldots \ldots \ldots \ldots$

2.2 Dilema do prisioneiro, jogador $2 \ldots \ldots \ldots \ldots$

2.3 Dilema do prisioneiro, forma bi-matricial. . . . . . . . . . . . . . . 10

2.4 Representação na forma normal, exemplo 1. . . . . . . . . . . . . . . . . . . 10

2.5 Representação normal da figura $2.3 \ldots$. . . . . . . . . . . . . . . . . . 14

2.6 Representação multi-agente da figura 2.3. . . . . . . . . . . . . . . . . . 15

2.7 Representação normal. . . . . . . . . . . . . . . . . . . . . . . 16

2.8 Exemplo de eliminação iterada de estratégias fracamente dominadas . . . . . . . . 17

2.9 Batalha dos sexos. . . . . . . . . . . . . . . . . 20 


\section{Capítulo 1}

\section{Introdução}

\subsection{Considerações preliminares}

Por uma teoria dos jogos diferenciais, entendemos um arcabouço teórico que se dedica ao estudo de situações de interação estratégica que dependem continuamente do tempo. Precisamente, esta dependência se dá através de uma equação diferencial ordinária. O objetivo dos jogadores é maximizar ou minimizar uma função de pagamento que também depende continuamente do tempo. Neste trabalho, abordamos jogos de soma zero, com dois jogadores.

A princípio, fazemos uma exposição dos elementos básicos da teoria dos jogos, discutindo tanto as formas de apresentação de um jogo como, e sobretudo, o seu conceito de solução. São apresentadas as formas normal, extensiva e Bayesiana, bem como as noções de equilíbrio de Nash, equilíbrio perfeito e equilíbrio sequencial. Discutimos ainda as condições sob as quais um jogo possui uma solução destas formas e quais as relações entre elas. As principais referências utilizadas são [44], [28], [43], [35] e [18].

Em seguida, damos início à discussão acerca dos jogos diferenciais. Expomos alguns exemplos desta classe de jogos e as definições formais associadas a este objeto. Em seguida, buscamos aplicações da Teoria do Controle Ótimo no sentido de resolver o jogo e obter as estratégias de cada jogador. Neste sentido, o primeiro passo é fornecer condições sob as quais o jogo possui uma função de valor, ou seja, tem uma solução. Isto garantido, se a função de valor $v: \Omega \longrightarrow \mathbb{R}$ for de classe $\mathcal{C}^{1}$, então, devido ao Princípio da Programação Dinâmica, ela será a solução clássica da equação não linear de primeira ordem de Hamilton-Jacobi-Isaacs (HJI) associada ao jogo e a obtenção dos controles ótimos se dará pelo método da retro-alimentação. Este capítulo tem como principais referências bibliográficas os trabalhos de [32], [46] e [24].

Entretanto, impor estas condições de regularidade à função de valor tem sido considerado muito restritivo pela literatura. Supondo que $v$ seja apenas contínua é possível demonstrarmos que $v$ será uma solução viscosa da equação Hamilton-Jacobi-Isaacs e novamente será possível recuperarmos os controles ótimos (estratégias) de cada jogador através da retro-alimentação. Por esta razão discutimos a teoria das soluções viscosas da Equação de Hamilton-Jacobi dedicando especial atenção a dois aspectos fundamentais de uma teoria de equações parciais: as propriedades gerais das soluções e suas condições de existência e unicidade. Os trabalhos de [2], [10], [16], [17] e [42] formam o corpo principal das referências para a discussão apresentada. 
Finalmente, retomamos a discussão da teoria dos jogos diferenciais propondo uma noção de solução baseada na teoria de soluções viscosas da equação de Hamilton-Jacobi-Isaacs e vemos que, sob hipóteses bastante gerais, um jogo diferencial sempre possui uma solução no sentido da viscosidade. Aqui, duas referências são cruciais. São elas [27] e [51].

Desta maneira, neste trabalho, expomos inicialmente alguns elementos da teoria dos jogos diferenciais de soma zero, com dois jogadores. Em seguida discutimos o Princípio da Programação Dinâmica e a teoria das soluções viscosas da equação de Hamilton-Jacobi. Por fim, aplicando este arcabouço teórico, analisamos a solução dos jogos diferenciais.

\subsection{Organização do trabalho}

O capítulo 2 introduz a teoria dos jogos através da exposição das principais formas de representação de um jogo e uma porção de exemplos. Em seguida, são discutidos três conceitos de solução: equilíbrio de Nash (EN), equilíbrio sequencial (ES) e equilíbrio perfeito (EP). São oferecidas condições de existência para cada uma destas soluções e é exposta a demonstração da inclusão $E P \subset E S \subset E N$.

No capítulo 3, apresentamos alguns exemplos e os elementos básicos da teoria dos jogos diferenciais, bem como seus resultados que nos serão pertinentes no decorrer do estudo. Em particular, oferecemos as condições sob as quais o jogo terá uma função de valor de acordo com Isaacs e uma primeira exposição da teoria dos jogos diferenciais segundo Friedman.

Já no capítulo 4, apresentamos a princípio, as já bem conhecidas aplicações do Princípio da Programação Dinâmica para um sistema controlável a um parâmetro, cuja função de valor é de classe $\mathcal{C}^{1}$. Apresentamos uma demonstração do PPD e em seguida o utilizamos para demonstrar que tal função de valor é solução da equação de Hamilton-Jacobi associada ao problema. Por fim, oferecemos a demonstração do procedimento de retro-alimentação.

Abrimos o capítulo 5 com uma discussão realmente breve acerca da relação entre a equação de Hamilton-Jacobi e as equações de Euler-Lagrange, resgatando em certo sentido a relevância daquelas equações no contexto da Mecânica e de problemas de otimização e cálculo variacional. Em seguida, apresentamos a teoria das soluções viscosas da equação de Hamilton-Jacobi. Através de diversos exemplos e conceitos, introduzimos esta noção em sua relação com as questões levantadas pela teoria das soluções fracas de equações diferenciais parciais e oferecemos a exposição de um teorema de existência e unicidade de soluções viscosas. Suas propriedades, sobretudo aquelas que são caras ao estudos da teoria dos jogos, são discutidas ao longo do capítulo.

Por fim, no capítulo 6, retomamos a discussão do jogo diferencial segundo Friedman e aplicamos a teoria das soluções viscosas da equação de Hamilton-Jacobi para oferecermos as condições sob as quais um jogo diferencial possui uma função de valor. Isto é feito ao expormos a demonstração do PPD para o caso de problemas de controle a dois parâmetros, o que é fortemente utilizado no estabelecimento do resultado que afirma que a função de valor de um jogo segundo Friedman é a solução viscosa da equação de Hamilton-Jacobi-Isaacs.

Finalmente, no apêndice ao trabalho, devido à sua importância no contexto elementar da teoria dos jogos, apresentamos uma demonstração auto-contida do Teorema do Ponto Fixo de Kakutani. 


\section{Capítulo 2}

\section{Noções elementares de teoria dos jogos}

Por teoria dos jogos, entendemos uma teoria matemática que aborda situações de interação estratégica. Em tal situação (jogo), indivíduos (jogadores) interagem e fazem escolhas de modo a determinar o resultado do jogo. Ao contrário do que ocorre com a teoria da escolha, nenhum jogador controla totalmente este resultado que depende das escolhas dos outros indivíduos.

Como uma teoria normativa, a teoria dos jogos tem por objetivo prescrever o que cada jogador deve escolher em cada jogo, com a finalidade de, através de sua influência parcial sobre o resultado final, obter aquele que o beneficie ao máximo possível. Em um sentido mais preciso, o objetivo da teoria dos jogos é obter a solução do jogo, caracterizando o comportamento esperado de cada indivíduo em cada situação estratégica.

Neste sentido, faremos algumas hipótese gerais sobre os indivíduos envolvidos em cada jogo. Supomos que os jogadores são racionais, no sentido de que possuem preferências sobre os possíveis resultados do jogo e escolherão suas ações de modo a obterem o melhor resultado de acordo com estas preferências. Mais ainda, supomos que os jogadores são inteligentes. Isto apenas significa que cada um deles entende o jogo pelo menos tão bem quanto nós.

Finalmente, observamos que, em geral, encontramos jogos de duas naturezas: cooperativos e não-cooperativos. O primeiro tipo aborda situações estratégicas em que jogadores escolhem suas ações de modo a maximizar um interesse alinhado, de forma a cooperar. O segundo tipo caracteriza uma classe de jogos na qual os indivíduos competem, tendo em vista objetivos não alinhados, ou, antagônicos. Neste trabalho abordaremos apenas jogos não-cooperativos. ${ }^{1}$

A principal motivação da teoria dos jogos reside na previsibilidade das ações de cada jogador no decorrer da interação estratégica, ou seja, o interesse da teoria dos jogos é o desenvolvimento de um arcabouço teórico segundo o qual possamos prever, ou melhor, predizer, qual o próximo passo, ou movimento, de cada jogador envolvido em determinado jogo.

Este interesse pelo desenvolvimento de uma teoria rigorosa que se ocupe da determinação dessas ações abre o trabalho seminal da área, devido a von Neumann ${ }^{2}$ [52]:

\footnotetext{
${ }^{1}$ Para uma introdução à teoria dos jogos cooperativos, ver [45] e [41]. Para uma exposição sobre a noção de solução e valor em jogos cooperativos ver o trabalho clássico de Shapley, [49]. Para aplicações das noções de solução para jogos cooperativos à Economia Matemática, ver [50], [13], [5], [9] e [6].

${ }^{2}$ Margittai von Neumann János Lajos nasceu em Budapeste, a 28 de dezembro de 1903. Trabalhou como professor nas Universidades de Berlim e de Hamburgo e, após migrar para os Estados Unidos, lecionou em Princeton, onde integrou o Instituto de Estudos Avançados. Contribuiu significativamente com o desenvolvimento da Física, sobretudo
} 
Die Frage, deren Beantwortung die vorliegende Arbeit anstrebt, ist die folgende: $n$

Spieler, $S_{1}, S_{2}, \ldots, S_{n}$, spielen ein gegebenes Gesellschaftsspiel $G$. Wie muss einer dieser

Spieler, $S_{m}$, spielen, um dabei ein möglichst günstiges Resultat zu erzielen?

É exatamente sobre o interesse em predizer a ação, ou estratégia, de um jogador, que reside a idéia de solução do jogo. Muitos exemplos de conceitos solução podem ser mencionados e dentre os mais relevantes estão a solução MinMax devida a von Neumann e Morgenstern [53] e a solução do equilíbrio de Nash [35].

Um dos primeiros refinamentos do conceito de equilíbrio de Nash, a noção de equilíbrio perfeito - bem como a estabilidade e técnicas de seleção de equilíbrios de Nash - podem ser encontrados no clássico livro de Harsanyi e Selten [18]. Já Myerson, [43], propõe um refinamento mais rigoroso, sugerindo a noção de equilíbrio próprio, e mostrando que os pontos de equilíbrio próprio de um jogo na forma normal constituem um subconjunto dos pontos de equilíbrio perfeito. Estas noções são analisadas em [54]. Recentemente, Halpern [28] sugeriu uma caracterização alternativa para estes conceitos, em verdade mais precisa, através da utilização de ferramentas da chamada nonstandard probability. Já Fudenberg e Levine, [7], abordam seleção de equilíbrios sob influência de reputação enquanto Kreps e Wilson, [19], fornecem a noção de equilíbrio sequencial para a solução de um jogo.

No que diz respeito às generalizações do teorema de existência ligado ao equilíbrio de Nash, Gliksberg, [25] oferece uma primeira extensão - no contexto de espaços de Hausdorff - ao passo que Reny, [47], discute o tema de forma quase exaustiva.

Neste capítulo exporemos as noções elementares da teoria dos jogos. Para tanto, iniciaremos nossa exposição discutindo jogos nas formas extensiva, normal e Bayesiana. Em seguida, introduziremos a noção de equilíbrio de Nash e apresentaremos um teorema de existência destes equilíbrios para o que definiremos como jogos finitos. Então, serão expostas algumas idéias de refinamentos do equilíbrio de Nash, bem como alguns resultados de existência para tais refinamentos.

\subsection{Principais modelos de jogos}

O primeiro passo para estudarmos um jogo é apresentarmos um modelo da situação de conflito a que este se refere. Neste sentido, nesta seção, vamos introduzir três modelos bastante tradicionais de jogos: a forma extensiva, a forma normal e a forma Bayesiana. Iniciamos a discussão com a forma extensiva, através de um exemplo.

\subsubsection{Forma extensiva}

Exemplo 1 Considere a seguinte situação: dois jogadores - 1 e 2 - depositam um dólar em um pote. De uma urna que contém 40 bolas brancas e 60 bolas vermelhas, o jogador 1 sorteia uma bola e observa sua cor. Em seguida ele decide entre duas ações: recuar e avançar.

Se ele recuar, então ele informa ao jogador 2 a cor sorteada e o jogo termina. Se a bola for branca, o jogador 1 recebe -1 dólar e o jogador 2 recebe 1 dólar. Já se a bola for vermelha, o

da Mecânica Quântica, da Lógica e da Teoria dos Conjuntos e da Economia Matemática, tendo integrado o Projeto Manhattan. Sob custódia militar, morreu em Washington, a 8 de fevereiro de 1957, vítima de um tumor cerebral. 
jogador 1 recebe 1 dólar e o jogador 2 recebe -1 dólar.

Se o jogador 1 decidir avançar, então o jogador 2 é chamado a jogar, e, sem conhecer a cor da bola sorteada, deverá escolher entre as ações enfrenta ou passa. Se ele passar, independente da cor da bola, o jogador 1 recebe 1 e o jogador 2 recebe -1. Por outro lado, se o jogador 2 enfrentar então os pagamentos de cada jogador dependerão da cor da bola sorteada. Se esta for branca, o jogador 1 ganha -2 e o jogador 2 ganha 2 e, se esta for vermelha, o jogador 1 recebe 2 ao passo em que o jogador 2 ganha -2 .

Esta situação pode ser modelada na forma extensiva de acordo com a seguinte figura:

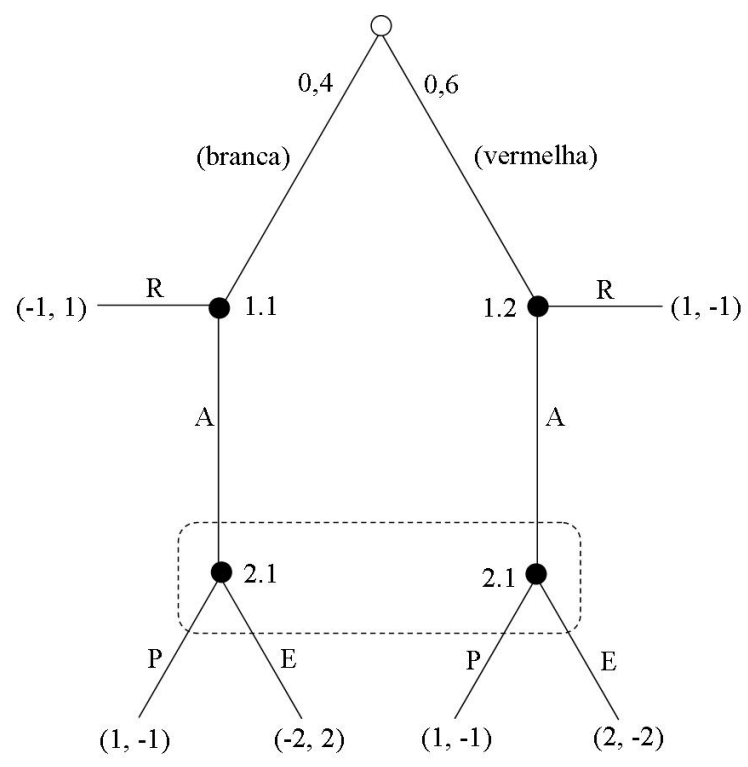

Figura 2.1: Exemplo de um jogo na forma extensiva.

Na figura 2.1 acima, o jogo se inicia em um nó branco, de onde partem duas arestas. Este nó branco representa o acaso - já que o jogador 1 sorteia uma bola na urna aleatoriamente. Cada aresta representa uma cor possível, e, a cada uma delas, está associada uma probabilidade - ao caso da bola ser de cor branca, associamos probabilidade 0, 4 e ao caso da bola ser de cor vermelha, associamos probabilidade 0,6. Em seguida a estas arestas encontramos dois nós pretos. Em cada um deles há um rótulo (1.1 e 1.2). Eles representam os dois estados de informação possíveis do jogador 1, ou seja: após ver a cor sorteada, o jogador 1 sabe que está no nó 1.1 se a bola for branca e no nó 1.2 se a bola for vermelha. De cada um destes nós partem duas novas arestas, às quais estão associadas as ações $A$ (avançar) ou $R$ (recuar). Ao final da aresta correspondente à ação $R$, encontram-se os pagamentos de cada jogador: um par ordenado onde a $i$-ésima entrada representa o pagamento do i-ésimo jogador.

Por outro lado, caso o jogador 1 avance, o jogo alcança outros dois nós, ambos denominados 2.1. Ambos os nós têm o mesmo nome e se encontram circundados por um caixa tracejada. Isto indica que o jogador 2 não tem informação suficiente para distinguir entre qualquer um dos nós 2.1 - isto porque não sabe a cor sorteada. Desta forma, ao passo que para o jogador 1 cada nó representava um conjunto de informação, para o jogador 2 ambos os nós representam o mesmo 
conjunto de informação e ele não consegue saber em qual nó o jogo está. A caixa tracejada indica um objeto chamado conjunto de informação do jogador 2. Novamente, seguem-se arestas de cada nó e, como elas encontram os chamados nós terminais (aqueles nos quais nenhum jogador, nem o acaso, é chamado a jogar) o jogo acaba. Novamente encontram-se os pagamentos.

Alguns comentários são importantes. Em primeiro lugar, os nós 1.1 e 1.2 não precisam conter as mesmas opções de ações para o jogador 1 . Isto porque, ao chegar a cada um destes nós, o jogador 1 saberá em qual deles se encontra: o conjunto de informação que contém cada um destes nós é unitário. Por outro lado, considere os nós no conjunto de informação 2.1 do jogador 2 . Como eles pertencem ao mesmo conjunto de informações, as alternativas que partem de cada um deles devem ser idênticas. Do contrário, o jogador 2 saberia distinguir em qual nó ele se encontra. Para ilustrarmos esta idéia, vejamos a figura 2.2. Nela, dois jogos estão representados na forma extensiva, com a diferença que, no segundo, o jogador 2, quando chamado a jogar, não sabe qual foi a ação do jogador 1, e, portanto, não sabe em qual nó do conjunto de informação 2.1 se encontra. Portanto, de cada um dos nós devem partir as mesmas alternativas. Já no primeiro jogo, 2 sabe exatamente em qual nó está. Logo se podem apresentar alternativas distintas, como $\{p, e\}$ em 2.1 e $\{P, E\}$ em 2.2 .

Em segundo lugar, gostaríamos que cada jogador tivesse, em cada conjunto de informação em que é chamado a jogar, condições de se recordar perfeitamente de toda a história do jogo até aquele instante. Ou seja, cada jogador, quando toma uma decisão sobre um conjunto de alternativas, recorda-se completamente de todas as suas decisões e das decisões passadas dos demais jogadores. Esta propriedade será chamada de recordação perfeita. ${ }^{3}$

Passemos às definições formais do jogo na forma extensiva. Para tanto, vejamos alguns elementos da teoria dos grafos.

Definição 1 Um grafo é um conjunto finito de pontos - ou nós - $\left\{x_{1}, \ldots, x_{k}\right\}$ e um conjunto de arestas - ou galhos - $\left\{g_{i j} \mid i, j=1, \ldots, k\right\}$ cada um dos quais conecta um par $\left\{x_{i}, x_{j}\right\}$ destes nós.

Cada um dos galhos de um grafo pode ser identificado de maneira biunívoca com os nós que conecta.

\section{Definição 2}

Trajeto Um trajeto é um conjunto de galhos da forma

$$
\left\{g_{i_{1} i_{2}}, \ldots, g_{i_{n-1} i_{n}}\right\}=\left\{g_{i_{m} i_{m+1}} \mid m=1, \ldots, n-1\right\}
$$

com $n \geq 2$. Este trajeto conecta os nós $x_{i_{1}}$ e $x_{i_{n}}$.

\footnotetext{
${ }^{3} \mathrm{Na}$ literatura em inglês, o termo utilizado é perfect recall. Por não encontrarmos em nenhum texto em português uma tradução para o termo, propomos esta, com base tanto no termo em inglês, como no significado da propriedade à qual o termo se refere.
} 

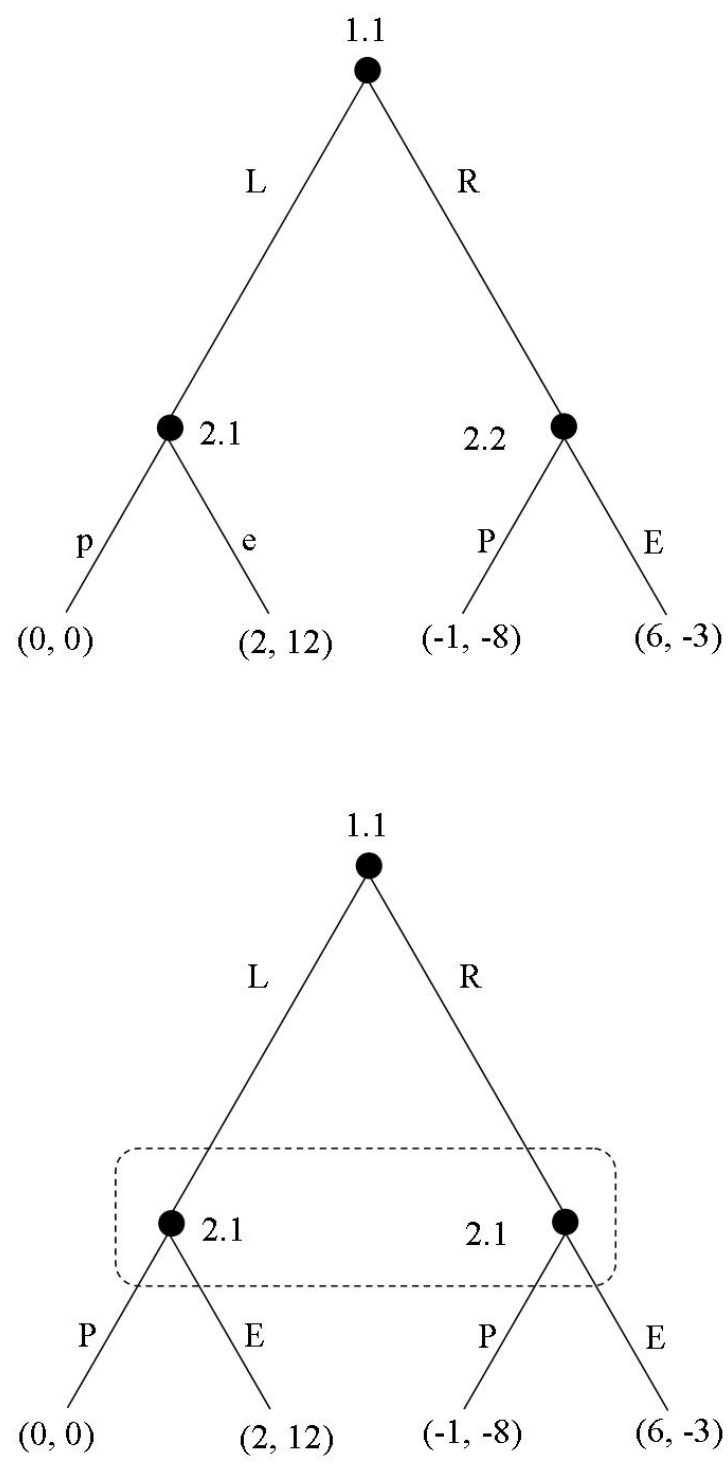

Figura 2.2: Exemplos de jogos com conjuntos de informação distintos.

Árvore Uma árvore é um grafo orientado no qual os nós são conectados por exatamente um trajeto. Uma árvore com raiz é qualquer árvore na qual um nó específico será designado como raiz da árvore e todos os trajetos partem deste nó.

Definição 3 Para qualquer $n \in \mathbb{N}$, um jogo de $n$ pessoas na forma extensiva é uma 7-upla

$$
\Gamma^{e}=\left\langle I, \mathcal{G}, L,\left(S_{i}\right)_{i \in I},\left(A_{i, s}\right)_{i \in I, s \in S_{i}},\left(\Theta_{i, s}\right)_{i \in I, s \in S_{i}},\left(u_{i}\right)_{i \in I}\right\rangle
$$

onde

1. I é o conjunto de jogadores $\{1,2,3, \ldots, n\}$,

2. $\mathcal{G}$ é uma árvore com raiz, 
3. L é um conjunto de rótulos que especifica qual jogador é chamado a jogar, qual o conjunto de informações e quais as alternativas disponíveis em cada nó de decisão. Além disso, nos chamados nós da natureza, especifica quais as possíveis alternativas juntamente com suas probabilidades,

4. $S_{i}$ é a coleção de todos os conjunto de informação de cada jogador $i$,

5. $A_{i, s}$ é o conjunto de alternativas disponíveis para o jogador $i$ no conjunto de informação $s \in S_{i}$,

6. $\left(\Theta_{i, s}\right)$ associa a cada conjunto de informação $s \in S_{i}$, um movimento em $A_{i, s}$,

7. $\left(u_{i}\right)_{i \in I}$ é uma coleção de funções pagamento que a cada nó terminal associa um pagamento a cada jogador $i$. Assim, se chamarmos de $\mathcal{T}$ o conjunto de todos nós terminais,

$$
u_{i}: \mathcal{T} \longrightarrow \mathbb{R}
$$

Desta forma, uma estratégia no jogo $\Gamma^{e}$ para o jogador $i$ é dada por $\Theta_{i}=\prod_{s \in S_{i}} \Theta_{i, s}$. Já o conjunto de estratégias de $i$ será denotado $A_{i}=\prod_{s \in S_{i}} A_{i, s}$.

$\mathrm{O}$ jogo $\Gamma^{e}$ será finito se a árvore do jogo, $\mathcal{G}$ for finita.

Observando nosso jogo no exemplo 1 acima, notamos que $I=\{1,2\}, S_{1}=\{1.1,1.2\}, S_{2}=\{2.1\}$, $A_{1}=\{R R, R A, A R, A A\}$ e $A_{2}=\{E, P\}$.

\subsubsection{Forma normal ou estratégica}

Uma maneira mais simples de representarmos um jogo é a chamada forma normal (ou estratégica). Nela, apenas especificamos um conjunto de jogadores, o conjunto de ações dentre as quais cada um pode escolher e uma função de pagamento para cada jogador. Formalmente,

Definição 4 Um jogo de n pessoas na forma normal é uma 3-upla

$$
\Gamma^{n}=\left\langle I,\left(C_{i}\right)_{i \in I},\left(u_{i}\right)_{i \in I}\right\rangle
$$

onde

1. I é o conjunto de jogadores,

2. $C_{i}$ é o conjunto de ações, ou estratégias puras, disponíveis para o jogador $i$ e

3. $u_{i}$ é a função pagamento, ou utilidade, de cada jogador $i$. De fato,

$$
u_{i}: \prod_{i \in I} C_{i} \longrightarrow \mathbb{R}
$$

Dizemos que $\Gamma^{n}$ é finito quando $I$ e $C_{i}$ forem conjuntos finitos, o último, para todo $i \in I$. 
A representação de um jogo na forma normal é mais simples do que a representação na forma extensiva e carrega a idéia de que, antes de iniciar o jogo, cada jogador é capaz de planejar todas as suas ações, ao contrário do anterior, onde o jogador pode decidir sobre suas ações dependendo do nó em que estiver quando for chamado a jogar.

Esta representação é freqüentemente dada em termos de matrizes. Vamos considerar aqui o caso de dois jogadores e introduzir a representação da forma normal através de um clássico exemplo devido a Albert Tucker, a saber, o dilema do prisioneiro.

Exemplo 2 (Dilema do Prisioneiro) Suponha que dois suspeitos de terem cometido um crime são detidos e colocados em salas separadas sem poderem se comunicar. Então a seguinte proposta é feita a cada um deles em separado: ambos têm a opção de confessar ou de não confessar o crime. Se ambos confessarem o crime, então cada um ficará preso 3 anos. Se nenhum dos dois confessar o crime, então ambos ficarão presos por 1 ano. E, caso um confesse e o outro não, o primeiro ficará preso por dois anos e o segundo por dez anos.

Nos termos da definição anterior, este jogo pode ser modelado da seguinte forma: $I=\{1,2\}$ é o conjunto de jogadores, representando cada um dos suspeitos. Para cada um deles o conjunto de ações possiveis, estratégias, é o mesmo, ou seja, $C_{1}=C_{2}=\{C, N C\}$, onde $C$ representa a ação confessa e NC representa a ação não confessa. Por fim, temos as funções utilidade de cada jogador, dadas por

$$
\begin{array}{cc}
u_{1}(C, C)=u_{2}(C, C)=-3 & u_{1}(N C, N C)=u_{2}(N C, N C)=-1 \\
u_{1}(C, N C)=u_{2}(N C, C)=-2 & u_{1}(N C, C)=u_{2}(C, N C)=-10 .
\end{array}
$$

Assim, todos os elementos de nosso jogo estão identificados com aqueles da nossa definição. Vamos agora representar o jogo. Isto se dará através de uma matriz. Vejamos nas tabelas 2.1 e 2.2 as matrizes para os jogadores 1 e 2 respectivamente:

\begin{tabular}{|c|c|c|}
\hline & $\mathrm{C}$ & $\mathrm{NC}$ \\
\hline $\mathrm{C}$ & -3 & -2 \\
$\mathrm{NC}$ & -10 & -1 \\
\hline
\end{tabular}

Tabela 2.1: Dilema do prisioneiro, jogador 1.

$e$

\begin{tabular}{|c|c|c|}
\hline & $\mathrm{C}$ & $\mathrm{NC}$ \\
\hline $\mathrm{C}$ & -3 & -10 \\
$\mathrm{NC}$ & -2 & -1 \\
\hline
\end{tabular}

Tabela 2.2: Dilema do prisioneiro, jogador 2. 
Como o jogo envolve apenas dois jogadores, é chamado de jogo bi-matricial, e, por conveniência, tem sido representado na literatura da seguinte forma:

\begin{tabular}{|c|c|c|}
\hline & $\mathrm{C}$ & $\mathrm{NC}$ \\
\hline $\mathrm{C}$ & $-3,-3$ & $-2,-10$ \\
$\mathrm{NC}$ & $-10,-2$ & $-1,-1$ \\
\hline
\end{tabular}

Tabela 2.3: Dilema do prisioneiro, forma bi-matricial.

Na tabela 2.3 vemos as estratégias de cada jogador. Nas linhas estão representadas as ações do jogador 1 e nas colunas, as ações do jogador 2. Em cada célula da tabela, encontramos um par de números. O primeiro deles é o pagamento do jogador 1 caso aquela combinação de estratégias seja jogada. O segundo deles é o pagamento do jogador 2 caso seja verificada aquela combinação de estratégias.

A representação na forma normal de um jogo explicita seus elementos de maneira bastante clara. As estratégias, as funções de pagamento, bem como o conjunto de jogadores, são dados. É bem verdade que as regras do jogo, em um sentido que veremos claramente adiante, são mais rigorosas do que na forma extensiva, uma vez que assumimos que os jogadores são capazes de escolher todos os seus movimentos antes de iniciar-se o jogo.

Para entendermos esta característica do jogo na forma normal, vamos modelar desta maneira a situação apresentada no exemplo 1 anterior. Temos então um conjunto de dois jogadores, $I=\{1,2\}$, um conjunto de estratégias para o jogador 1 dado por $C_{1}=\{R R, R A, A R, A A\}$ e um conjunto de estratégias para o jogador 2 dado por $C_{2}=\{P, E\}$. Como o acaso está presente no jogo, a função de pagamento de cada jogador depende das probabilidades do jogador 1 sortear uma bola branca ou vermelha. Assim, utilizando a definição da função de utilidade von Neumann-Morgenstern ${ }^{4}$ temos os pagamentos exibidos na tabela 2.4 .

\begin{tabular}{|c|c|c|}
\hline & $\mathrm{P}$ & $\mathrm{E}$ \\
\hline $\mathrm{RR}$ & $1,-1$ & 0,0 \\
$\mathrm{RA}$ & 0,0 & $1 / 2,-1 / 2$ \\
$\mathrm{AA}$ & 0,0 & 0,0 \\
$\mathrm{AR}$ & $1,-1$ & $-1 / 2,1 / 2$ \\
\hline
\end{tabular}

Tabela 2.4: Representação na forma normal, exemplo 1.

Observe que, nesta situação, o jogador 1 deve escolher seus movimentos antes de saber qual a cor sorteada.

De acordo com von Neumann-Morgenstern, a análise de um jogo, tanto na forma extensiva, como na forma estratégica, pode ser efetuada apenas observando-se sua representação normal. Isto

\footnotetext{
${ }^{4}$ Esta noção pode ser encontrada no apêndice ao livro clássico destes autores [53] ou no texto mais recente devido a Mas-Colell, Whinston e Green [1].
} 
se baseia na hipótese - que veremos bastante forte - de que os jogadores, ao observarem um jogo, são capazes de formular suas ações, ou estratégias, ao começo do jogo, independentemente dos demais jogadores. Desta maneira, cada jogo na forma extensiva possui uma representação normal, bem como o jogo na forma estratégica, e, analisarmos estas situações, dependeria apenas desta mesma representação normal.

Na verdade, von Neumann-Morgenstern propõem uma aplicação do conjunto dos jogos na forma extensiva sobre o conjunto dos jogos na forma normal.

Note que em todos os exemplos acima, ambos os jogadores possuíam as mesmas informações no instante inicial do jogo. Ou seja, quando o jogador 1 no primeiro exemplo inicia o jogo ele ainda não sabe a cor da bola sorteada, posto que ainda não ocorreu o sorteio. Por outro lado, nenhum dos suspeitos do segundo exemplo possuem informações privilegiadas sobre o comportamento do outro ou sobre qualquer das regras do jogo. Neste sentido, ambos são chamados de jogos com informação completa. Ou seja, nenhum dos jogadores possui qualquer informação privada que não esteja disponível aos demais no início do jogo.

Isto não seria verdadeiro se o jogador 1 soubesse a cor sorteada já quando o jogo se inicia para os dois jogadores. Ou se um dos suspeitos no dilema do prisioneiro soubesse que o outro escolheria com certeza uma das alternativas. Em casos onde alguns jogadores possuem informações privadas que não estão disponíveis para os demais jogadores, dizemos que se trata de um jogo com informação incompleta e chamamos a estas informações privadas de tipos do jogador.

Ao considerarmos um jogo na forma extensiva, e tentarmos representá-lo na forma normal, seguindo a noção de von Neumann-Morgenstern, utilizamos a hipótese de que os jogadores são capazes de formular suas ações antes do jogo começar. Entretanto, no caso em que a informação é incompleta isto não é verdadeiro. O motivo para isto é relativamente simples: no caso da informação incompleta, cada jogador deverá escolher suas ações com base em seus tipos privados, o que ele apenas saberá assim que o jogo começar - logo, os jogadores não serão capazes de formular planos sobre o jogo antes ou no instante em que este se inicia.

Neste sentido, Harsanyi sugeriu uma generalização da forma extensiva, chamada forma Bayesiana, para representarmos os jogos com informação incompleta. Este será o assunto da próxima subseção.

\subsubsection{Forma Bayesiana}

A definição de um jogo na forma Bayesiana se assemelha largamente àquela para a forma estratégica.

Como naquele caso, temos um conjunto de jogadores $I$, um conjunto de possíveis ações para cada um dos jogadores $C_{i}$ e uma função de pagamento $u_{i}$. Entretanto, agora, teremos o conjunto dos possíveis tipos privados - ou tipos - de cada jogador, denotado por $T_{i}$ e a função de probabilidade $p_{i}$.

Consideremos dois conjuntos

$$
C_{-i}=\prod_{j \in I \backslash\{i\}} C_{j}
$$


$\mathrm{e}$

$$
T_{-i}=\prod_{j \in I \backslash\{i\}} T_{j}
$$

juntamente com

$$
C=\prod_{i \in I} C_{i}
$$

$\mathrm{e}$

$$
T=\prod_{i \in I} T_{i}
$$

Então, se denotarmos por $\Delta\left(T_{-i}\right)$ o conjunto das distribuições de probabilidade sobre $T_{-i}$, temos que

$$
p_{i}: T_{i} \longrightarrow \Delta\left(T_{-i}\right) \text {. }
$$

Assim, para cada tipo do jogador $i$, ou seja, cada informação privada que o jogador $i$ possua, ele associa uma distribuição de probabilidade sobre o conjunto das informações privadas dos demais jogadores. Isto significa que se o tipo de $i$ for $t_{i}$ então ele empregará a distribuição de probabilidades $p_{i}\left(\cdot \mid t_{i}\right)$ e o número $p_{i}\left(t_{-i} \mid t_{i}\right)$ é a probabilidade atribuída por $i$ ao tipo $t_{-i}$ quando seu tipo for de fato $t_{i}$.

Já a função de pagamento do jogo dependerá tanto das escolhas de cada jogador como de seus tipos e, desta maneira, será uma aplicação

$$
u_{i}: C \times T \longrightarrow \mathbb{R}
$$

Estamos agora em condições de definir o jogo na forma Bayesiana.

Definição 5 Um jogo na forma Bayesiana é uma 5-upla

$$
\Gamma^{b}=\left\langle I,\left(C_{i}\right)_{i \in I},\left(T_{i}\right)_{i \in I},\left(p_{i}\right)_{i \in I},\left(u_{i}\right)_{i \in I}\right\rangle
$$

onde cada elemento é como na discussão anterior.

O jogo $\Gamma^{b}$ será dito finito se os conjuntos $I, C_{i}$ e $T_{i}$ forem finitos para todo $i \in I$.

Novamente tomando em consideração nosso jogo do exemplo 1, façamos a suposição de que o jogador 1 sabe a cor sorteada antes de o jogo ser iniciado. Isto tornaria a situação em questão em um exemplo de jogo com informação incompleta e, portanto, a forma Bayesiana seria uma maneira mais adequada de modelá-lo. Neste caso, teremos que $I=\{1,2\}, C_{1}=\{R R, R A, A R, A A\}$ e $C_{2}=\{E, P\}$.

Entretanto, agora o jogador 1 possui dois tipos possíveis, que denotaremos como os nós em que ele se encontraria na ocasião de cada um. Assim, $T_{1}=\{1.1,1.2\}$ e $T_{2}=\{2.1\}$. Já as funções de probabilidade em cada caso serão dadas por 


$$
\begin{array}{cc}
p_{1}(2.1 \mid 1.1)=1 & p_{1}(2.1 \mid 1.2)=1 \quad e \\
p_{2}(1.1 \mid 2.1)=0,4 & p_{2}(1.2 \mid 2.1)=0,6 .
\end{array}
$$

As funções de pagamento do jogo oferecem os mesmos pagamentos discutidos na figura 2.4.

Como mencionamos no início deste capítulo, o interesse da teoria dos jogos recai infalivelmente sobre a prescrição do comportamento racional de cada jogador, ou seja, na obtenção da solução do jogo, procurando responder como esperamos que cada jogador escolha suas ações em cada contexto. Com esta finalidade em nosso horizonte, vamos indicar na próxima subseção como um jogo na forma extensiva pode ser representado além da forma normal - a chamada forma multi-agente - e, mais ainda, como representar a forma Bayesiana através da forma normal.

Assim, procuraremos caracterizar as soluções de jogos na forma normal e, desta maneira, poderemos caracterizar as soluções das três formas de jogo discutidas acima.

\subsubsection{Representações multi-agente e tipo-agente}

Em um jogo na forma extensiva, cada jogador $i \in I$ possui um conjunto $S_{i}$ de conjuntos de informação e, ao representarmos um jogo extensivo na forma normal supomos que o mesmo jogador escolhe uma ação que prescreve movimentos em cada um dos seus conjuntos de informação, mesmo que estes não sejam de fato alcançados pelo trajeto do jogo.

Na representação multi-agente de um jogo na forma extensiva, o conjunto de jogadores não será mais $I$, mas será dado por $S^{*}=\prod_{i \in I} S_{i}$ - um conjunto finito se o jogo inicial for finito. Assim, cada jogador em $I$ será representado em cada um dos seus conjuntos de informação por um jogador diferente em $S^{*}$ que desfruta de suas mesmas preferências sobre os resultados possíveis do jogo. Os elementos de $S^{*}$ podem ser chamados de jogadores temporários, ou agentes temporários. Ao invés de supor que os jogadores em $I$ planejam suas ações para todo o jogo, supomos agora que os jogadores em $S^{*}$ são os que tomam decisões sobre o jogo antes deste ser iniciado.

Vamos definir formalmente os elementos que caracterizam um jogo extensivo na forma multiagente. Para qualquer jogador $i \in I$ e para todo conjunto de informações $s \in S_{i}$ seja $A_{i, s}$ o conjunto de todos os movimentos possíveis. Neste caso, $A_{s}$ é o conjunto de ações disponíveis para o jogador temporário $s$. Cada jogador temporário tem uma função de pagamento $v_{s}$ que é definida como segue: para todo $i \in I$ e para todo $s \in S_{i}$

$$
v_{s}: \prod_{r \in S^{*}} A_{r} \longrightarrow \mathbb{R}
$$

é tal que para qualquer $\left(a_{s}\right)_{s \in S^{*}} \in \prod_{s \in S^{*}} A_{s}$, se o perfil de estratégias para a representação normal for $\left(c_{j}\right)_{j \in I}$ tal que $c_{j}(r)=a_{r}$ para todo $j \in I$ e para todo $r \in S_{j}$, então deve valer

$$
v_{s}\left(\left(a_{r}\right)_{r \in S^{*}}\right)=u_{i}\left(\left(c_{j}\right)_{j \in I}\right) .
$$


Assim, o jogo extensivo modelado na forma multi-agente é composto pela seguintes estruturas reunidas na 3 -upla $\left\langle S^{*},\left(A_{s}\right)_{s \in S^{*}},\left(v_{s}\right)_{s \in S^{*}}\right\rangle$. Para ilustrarmos esta forma de representação, e apontarmos em que medida ela pode diferir da representação normal, vamos utilizar um exemplo. Considere o jogo na forma extensiva como na figura 2.3.

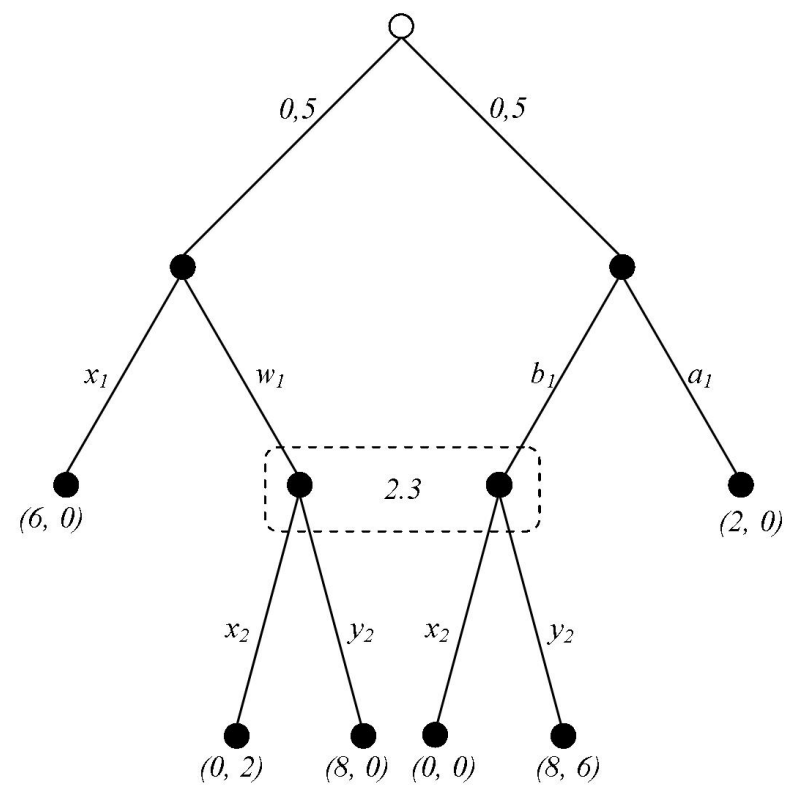

Figura 2.3: Representação, forma extensiva.

A representação normal deste jogo é composta pelos elementos $I=\{1,2\}$ e os conjuntos de estratégias $C_{1}=\left\{a_{1} w_{1}, a_{1} x_{1}, b_{1} w_{1}, b_{1} x_{1}\right\}$ e $C_{2}=\left\{y_{2}, z_{2}\right\}$. Já os pagamentos são mostrados na tabela 2.5 .

\begin{tabular}{|c|c|c|}
\hline & $\mathrm{y}_{2}$ & $\mathrm{z}_{2}$ \\
\hline $\mathrm{a}_{1} w_{1}$ & 5,0 & 1,1 \\
$\mathrm{a}_{1} x_{1}$ & 4,0 & 4,0 \\
$\mathrm{~b}_{1} w_{1}$ & 8,3 & 0,1 \\
$\mathrm{~b}_{1} x_{1}$ & 7,3 & 3,0 \\
\hline
\end{tabular}

Tabela 2.5: Representação normal da figura 2.3.

Por outro lado, se considerarmos a representação multi-agente deste jogo, temos o conjunto de jogadores $S^{*}=\{1.1,1.2,2.1\}$, os conjuntos de estratégias $D_{1.1}=\left\{a_{1}, b_{1}\right\}, D_{1.2}=\left\{w_{1}, x_{1}\right\}$ e $D_{2.1}=\left\{y_{2}, z_{2}\right\}$. As funções de pagamento dos três jogadores envolvidos estão dispostas na tabela 2.6.

Para apreciarmos a diferença técnica entre ambas estas representações aguardaremos até introduzirmos a noção de estratégia dominada, fraca e estritamente, e então retomaremos este exemplo.

Enquanto a representação multi-agente oferece uma forma estratégica do jogo dado na forma extensiva, a representação tipo-agente aborda a forma Bayesiana de jogo com informação incompleta. 


\begin{tabular}{|c|c|c|c|c|c|c|c|}
\hline & $\mathrm{w}_{1}$ & & $\mathrm{y}_{2}$ & & $\mathrm{w}_{1}$ & & $\mathrm{z}_{2}$ \\
\hline $\mathrm{a}_{1}$ & $5,5,0$ & & $4,4,0$ & $1,1,1$ & & $4,4,0$ \\
$\mathrm{~b}_{1}$ & $8,8,3$ & & $7,7,3$ & $0,0,1$ & & $3,3,0$ \\
\hline
\end{tabular}

Tabela 2.6: Representação multi-agente da figura 2.3.

Apresentaremos agora esta abordagem, devida a Harsanyi [29].

No jogo na forma tipo-agente, chamado originalmente por Harsanyi de Jogo de Selten - por este ter sugerido a abordagem - existe um jogador para cada tipo possível no jogo Bayesiano. Assim, o conjunto de jogadores é dado por $T^{*}=\bigcup_{i \in I} T_{i}$. Como cada tipo-agente em $T^{*}$ possui as mesmas alternativas de ações que o jogador $i$ que representa, as ações disponíveis para cada $t_{i, j} \in T^{*}$ são dadas por $D_{t_{i, j}}=C_{i}$.

A interpretação para cada jogador $t_{i, j} \in T^{*}$ é que $t_{i, j}$ joga como se o tipo de $i$ fosse de fato $t_{i, j}$, o $j$-ésimo tipo possível em alguma ordenação - uma vez que $T_{i}$ é finito.

Já a função de pagamento no jogo tipo-agente é dada pela aplicação

$$
v_{t_{i, j}}: \prod_{s \in T^{*}} A_{s} \longrightarrow \mathbb{R}
$$

que associa a todo $a=(a(s))_{s \in T^{*}} \in A_{s}$ o seguinte número

$$
v_{t_{i}}(a)=\sum_{t_{-i} \in T_{-i}} p_{i}\left(t_{-i} \mid t_{i}\right) u_{i}\left(a\left(t_{j}\right)_{j \in I},\left(t_{j}\right)_{j \in I}\right)
$$

Assim sendo, o jogo na forma Bayesiana pode ser escrito na forma estratégica através dos elementos que compõem a 3-upla $\left\langle T^{*},\left(A_{r}\right)_{r \in T^{*}},\left(v_{r}\right)_{r \in T^{*}}\right\rangle$.

Desta maneira, notamos que tanto um jogo na forma extensiva, como um jogo na forma Bayesiana podem ser escritos, e representados, como um jogo na forma estratégica, ou normal. Nossa próxima seção se ocupará de expor os principais conceitos de solução de um jogo na forma normal - dentre eles o chamado equilíbrio de Nash. No que diz respeito aos refinamentos da noção de equilíbrio, retomaremos a forma extensiva.

\subsection{Soluções do jogo: o equilíbrio de Nash}

Como já fora mencionado, o interesse da teoria dos jogos é prescrever o comportamento racional de cada jogador em cada jogo. Nesta seção desenvolveremos algumas noções de solução e, a mais importante delas, a noção de Equilíbrio de Nash. Por enquanto, nos limitaremos à discussão acerca dos jogos na forma estratégica. Retomaremos os jogos na forma extensiva quando abordarmos os refinamentos do equilíbrio. 


\subsubsection{Estratégias dominadas e o jogo residual}

Iniciamos nossa discussão de posse de um jogo na forma normal $\Gamma^{n}$ onde $I=\{1,2\}, C_{1}=\{A, B\}$, $C_{2}=\{a, b\}$ e, cujas funções pagamentos são representadas na tabela 2.7.

\begin{tabular}{|c|c|c|}
\hline & $\mathrm{a}$ & $\mathrm{b}$ \\
\hline $\mathrm{A}$ & 1,2 & 3,7 \\
$\mathrm{~B}$ & 9,1 & 6,4 \\
\hline
\end{tabular}

Tabela 2.7: Representação normal.

Observemos que, se o jogador 1 escolher a estratégia $B$ e o jogador 2 escolher a estratégia $a$ então o jogador 1 receberá 9 ao invés de 1 que receberia caso escolhesse $A$. Ainda escolhendo $B$, o jogador 1 ganhará 6 caso 2 escolha $b$. Em termos dos elementos do jogo temos:

$$
u_{1}(A, a)=1<u_{1}(B, a)=9 \quad u_{1}(A, b)=3<u_{1}(B, b)=6 .
$$

Por outro lado, vemos que o mesmo ocorre com o jogador 2 :

$$
u_{2}(A, a)=2<u_{2}(A, b)=7 \quad u_{2}(B, a)=1<u_{2}(B, b)=4 .
$$

Assim, nota-se que, independente do que 1 faça, é melhor para 2 jogar $b$, assim como, a despeito do que 2 jogue, o jogador 1 se beneficia mais se jogar $B$. Desta maneira, observamos que para o jogador 1 a estratégia $B$ domina a estratégia $A$ e, para o jogador 2, a estratégia $b$ domina $a$.

É razoável supormos que um jogador racional não deverá jogar uma estratégia dominada, posto que isto contraria a possibilidade dele obter um pagamento mais alto. Desta maneira podemos eliminar estratégias que são dominadas do conjunto daquelas que esperamos utilizadas.

Neste sentido, se eliminarmos as estratégias dominadas de ambos os jogadores, apenas o perfil $(B, b)$ permanece e, assim, devemos esperar que este fosse o perfil utilizado pelos jogadores.

Formalmente temos a definição

Definição 6 (Estratégias dominadas) Dizemos que a estratégia $c_{i, j} \in C_{i}$ é fortemente, ou estritamente, dominada para o jogador $i$ se existir $c_{*} \in C_{i}$ tal que

$$
u_{i}\left(c_{-i}, c_{i, j}\right)<u_{i}\left(c_{-i}, c_{*}\right)
$$

para todo perfil $c_{-i} \in C_{-i}$.

Dizemos que a estratégia $c_{i, j} \in C_{i}$ é fracamente dominada para o jogador $i$ se existir $c_{*} \in C_{i}$ tal que

$$
u_{i}\left(c_{-i}, c_{i, j}\right) \leq u_{i}\left(c_{-i}, c_{*}\right)
$$


para todo perfil $c_{-i} \in C_{-i}$.

Consideremos o jogo $\Gamma^{n}=\left\langle I,\left(C_{i}\right)_{i \in I},\left(u_{i}\right)_{i \in I}\right\rangle$ e para cada jogador $i \in I$ defina $C_{i}^{1}$ como o sub-conjunto das estratégias em $C_{i}$ que não são dominadas fortemente. Assim, obtemos o jogo

$$
\Gamma^{n, 1}=\left\langle I,\left(C_{i}^{1}\right)_{i \in I},\left(u_{i}^{1}\right)_{i \in I}\right\rangle
$$

onde

$$
u_{i}^{1}=\left.u_{i}\right|_{\prod_{i \in I} C_{i}^{1}}
$$

É fácil vermos - por ser $C_{i}$ finito para todo $i \in I$ - que, para todo $k \in \mathbb{N}, C_{i}^{k+1} \subset C_{i}^{k}$ e que $C_{i}^{k} \neq \emptyset$. Mais ainda, existe um $K \in \mathbb{N}$ tal que $C_{i}^{K}=C_{i}^{K+1}=\ldots=C_{i}^{K+n}=\ldots$ para todo $i \in I$ e para todo $n \in \mathbb{R}$.

Definição 7 Chamaremos ao jogo $\Gamma^{n, K}=\left\langle I,\left(C_{i}^{K}\right)_{i \in I},\left(u_{i}^{K}\right)_{i \in I}\right\rangle$ de jogo residual e o denotaremos por $\Gamma^{n, \infty}$. Ao processo de obtenção do jogo residual, chamamos eliminação iterada de estratégias dominadas.

Note que o jogo residual não contém nenhuma estratégia fortemente dominada.

Por outro lado, a eliminação iterada de estratégias fracamente dominadas não é um procedimento tão simples quanto o descrito acima para o caso da dominação forte. Isto porque a ordem de eliminação é crucial na determinação do resultado do jogo. Vejamos um exemplo.

Considere o jogo da tabela 2.8. Nele, $I=\{1,2\}, C_{1}=\left\{x_{1}, y_{1}, z_{1}\right\}, C_{2}\left\{x_{2}, y_{2}\right\}$. As funções de pagamentos avaliadas nos possíveis perfis de estratégias estão dispostas na tabela 2.8.

\begin{tabular}{|c|c|c|}
\hline & $\mathrm{x}_{2}$ & $\mathrm{y}_{2}$ \\
\hline $\mathrm{x}_{1}$ & 6,4 & 8,4 \\
$\mathrm{y}_{1}$ & 4,5 & 5,3 \\
$\mathrm{z}_{1}$ & 3,3 & 2,4 \\
\hline
\end{tabular}

Tabela 2.8: Exemplo de eliminação iterada de estratégias fracamente dominadas

Claramente, a estratégia $x_{1}$ domina fortemente $y_{1}$ e $z_{1}$. Suponhamos que $y_{1}$ seja eliminada primeiro. Então, $x_{2}$ é fracamente dominada por $y_{2}$, e, portanto, ao eliminarmos $x_{2}$, o resultado do jogo deverá ser $\left(x_{1}, y_{2}\right)$. Se, por outro lado, eliminarmos primeiramente $z_{1}$, então será $x_{2}$ que dominará $y_{2}$. Assim, o resultado esperado será $\left(x_{1}, x_{2}\right)$.

Este exemplo indica que é preciso termos cuidado com a eliminação iterada de estratégias fracamente dominadas.

Por outro lado, é possível encontrarmos jogos, como no caso do dilema do prisioneiro, em que não existem estratégias dominadas. Outro conceito de solução para um jogo é necessário. É neste sentido que introduziremos o conceito do equilíbrio de Nash (EN). 


\subsubsection{O Equilíbrio de Nash: idéias iniciais, existência e não-unicidade}

Comecemos com uma definição

\section{Definição 8 (Estratégia mista e perfil de estratégias mistas)}

Estratégia mista Dado um jogo na forma estratégica, $\Gamma^{n}=\left\langle I,\left(C_{i}\right)_{i \in I},\left(u_{i}\right)_{i \in I}\right\rangle$, uma estratégia mista para o jogador $i$ é uma distribuição de probabilidades sobre $C_{i}$. Denotamos por $\Delta\left(C_{i}\right)$ o conjunto de todas as distribuições de probabilidades sobre $C_{i}$.

Perfil de estratégias mistas Um perfil de estratégias mistas $\sigma \in \prod_{i \in I} \Delta\left(C_{i}\right)$ é um vetor que associa a cada jogador uma estratégia mista.

Uma estratégia mista $\overline{\sigma_{i}}$ pode ser entendida como uma função que, a cada possível ação do jogador $i \in I$ associa um número não negativo entre 0 e 1 e, cuja soma indexada pelos elementos de $C_{i}$ é igual a 1 ; formalmente:

$$
\begin{aligned}
\overline{\sigma_{i}}\left(c_{i}^{j}\right) & \geq 0 \\
\sum_{c_{i}^{j} \in C_{i}} \overline{\sigma_{i}}\left(c_{i}^{j}\right) & =1 .
\end{aligned}
$$

Desta forma, ao invés do jogador $i$ escolher entre uma estratégia daquelas em $C_{i}$ ele escolherá uma distribuição de probabilidades em $\Delta\left(C_{i}\right)$ que, a cada elemento de $C_{i}$ atribui uma probabilidade. Entenderemos que, se o jogador $i$ jogar uma estratégia pura $c_{i}^{j} \in C_{i}$ então isto será como se ${ }^{5}$ ele jogasse a distribuição de probabilidade $\bar{\sigma}_{i}$ tal que $\bar{\sigma}_{i}\left(c_{i}^{j}\right)=1$ e $\bar{\sigma}_{i}\left(c_{i}^{l}\right)=0$ para todo $l \neq j$. Eventualmente, denotaremos esta estratégia mista como $\left[c_{i}^{j}\right]$.

Assim, a partir de agora, o termo estratégias será utilizado em referência a estratégias mistas, enquanto que estratégias puras serão um caso particular das primeiras como comentado acima.

Isto discutido, iniciaremos de fato nossa exposição acerca do equilíbrio de Nash. A idéia por trás desta noção é que todos os indivíduos envolvidos em uma situação de interação estratégica ganham quando fazem o que é melhor para si dado o que os demais indivíduos escolhem. Ou seja, cada jogador deve jogar a sua melhor resposta para a estratégia dos demais indivíduos. ${ }^{6}$

Suponha que em um determinado jogo $\Gamma^{n}=\left\langle I,\left(\Delta\left(C_{i}\right)\right)_{i \in I},\left(u_{i}\right)_{i \in I}\right\rangle$ o perfil de estratégias $\sigma^{*}=\left(\sigma_{1}^{*}, \sigma_{2}^{*}, \ldots, \sigma_{n}^{*}\right)$ seja tal que

$$
u_{i}\left(\sigma_{-i}^{*}, \sigma_{i}^{*}\right) \geq u_{i}\left(\sigma_{-i}^{*}, \sigma_{i}\right) \quad \forall \sigma_{i} \in \Delta\left(C_{i}\right) \quad \forall i \in I
$$

\footnotetext{
${ }^{5} \mathrm{O}$ destaque vem por conta da relevância da hipótese as if no argumento em Economia Matemática.

${ }^{6} \mathrm{~A}$ idéia de que cada indivíduo deve escolher a ação que maximiza seus interesses tomando em conta as ações dos demais indivíduos complementa a idéia anterior, marcante do Liberalismo e presente na fundação do Pensamento Econômico desde Smith, de que os indivíduos devem maximizar seus interesses independente das ações que lhe são alheias.
} 
Então, $\sigma^{*}$ é um perfil tal que, uma vez atingido pelos jogadores, nenhum deles terá incentivo a se desviar, posto que, qualquer desvio com relação a este perfil implicará um pagamento menor. Assim, trata-se de um perfil de estratégias de estado estacionário.

Outra maneira de descrevermos tal estratégia é pensarmos na correspondência de melhor resposta do jogo.

Definição 9 Considere o jogo $\Gamma^{n}\left\langle I,\left(\Delta\left(C_{i}\right)\right)_{i \in I},\left(u_{i}\right)_{i \in I}\right\rangle$. Então, a correspondência de melhor resposta do jogador $i$ é a aplicação

$$
\mathcal{B}_{i}: \prod_{j \in I \backslash i} \Delta\left(C_{j}\right) \longrightarrow \Delta\left(C_{i}\right)
$$

dada por

$$
\mathcal{B}_{i}\left(\sigma_{-i}\right)=\left\{\sigma_{i} \in \Delta\left(C_{i}\right) \mid u_{i}\left(\sigma_{-i}, \sigma_{i}\right) \geq u_{i}\left(\sigma_{-i}, \sigma_{i}^{\prime}\right) \quad \forall \sigma_{i}^{\prime} \in \Delta\left(C_{i}\right)\right\}
$$

Suponha agora que $\sigma^{*}$ seja um perfil de estratégias de estado estacionário. É fácil notarmos que

$$
\sigma_{i}^{*} \in \mathcal{B}_{i}\left(\sigma_{-i}^{*}\right)
$$

para todo $i \in I$.

Assim, se definirmos a correspondência de melhor resposta do jogo como

$$
\mathcal{B}(\sigma)=\mathcal{B}_{1}\left(\sigma_{-1}\right) \times \mathcal{B}_{2}\left(\sigma_{-2}\right) \times \ldots \times \mathcal{B}_{n}\left(\sigma_{-n}\right)
$$

então é claro que

$$
\sigma^{*} \in \mathcal{B}\left(\sigma^{*}\right)
$$

Isto implica que um perfil de estratégias de estado estacionário é um ponto fixo da correspondência de melhor resposta do jogo. É fácil vermos que um ponto fixo da correspondência de melhor resposta do jogo é também um ponto de equilíbrio estacionário. Logo, ambas as noções são equivalentes.

Desta discussão apreendemos que um equilíbrio do qual nenhum jogador tem incentivo a desviar é, ao mesmo tempo, a melhor resposta de cada jogador ao que os demais estão jogando. Esta noção de solução de jogo é devida a Nash e ficou conhecida na literatura como o conceito de equilíbrio que leva seu nome.

Definição 10 (Equilíbrio de Nash [35]) Considere um jogo $\Gamma^{n}=\left\langle I,\left(\Delta\left(C_{i}\right)\right)_{i \in I},\left(u_{i}\right)_{i \in I}\right\rangle$. Dizemos que um perfil de estratégias $\sigma^{*} \in \prod_{i \in I} \Delta\left(C_{i}\right)$ é um Equilíbrio de Nash para $\Gamma^{n}$ se for um equilíbrio de estado estacionário ou, o que é equivalente, um ponto fixo da correspondência de melhor resposta do jogo.

No restante desta seção, vamos discutir a existência de equilíbrios de Nash, sua não unicidade e indicar algumas de suas aplicações. Antes disso, no entanto, examinaremos alguns exemplos. 
Retomemos a análise do dilema do prisioneiro. O jogo é representado na tabela 2.3. Apesar deste jogo não ter nenhuma estratégia estritamente dominada para nenhum dos jogadores, ele possui equilíbrio de Nash em estratégias puras. Vejamos:

$$
\begin{array}{cc}
\mathcal{B}_{1}(C)=\{C\} & \mathcal{B}_{1}(N C)=\{C\} \\
\mathcal{B}_{2}(C)=\{C\} & \mathcal{B}_{2}(N C)=\{C\} .
\end{array}
$$

Assim, se tomarmos a correspondência de melhor resposta do jogo

$$
\mathcal{B}=\mathcal{B}_{1} \times \mathcal{B}_{2}
$$

temos que

$$
\begin{aligned}
\mathcal{B}(N C, N C) & =(C, C) \\
\mathcal{B}(N C, C) & =(C, C) \\
\mathcal{B}(C, C) & =(C, C) \\
\mathcal{B}(C, N C) & =(C, C) .
\end{aligned}
$$

O único ponto fixo da correspondência de melhor resposta, e, portanto, o único equilíbrio de Nash do jogo, é dado pelo perfil $(C, C)$.

Um jogo pode não ter um único equilíbrio de Nash.

Exemplo 3 (Batalha dos Sexos) Considere a seguinte situação: um casal deseja se encontrar em uma noite de sábado e há duas opções disponíveis. Eles podem ir ao Ballet (B) ou ao Futebol (F). O homem - que chamaremos de jogador 1 - prefere ir ao Futebol. Já a mulher, jogador 2, prefere ir ao Ballet. Infelizmente ambos estão impedidos de se comunicar. Os pagamentos de ambos seguem na tabela 2.9. Claramente ambos preferem sair juntos a separados. Os pagamentos

\begin{tabular}{|c|c|c|}
\hline & $\mathrm{F}$ & $\mathrm{B}$ \\
\hline $\mathrm{F}$ & 3,1 & 0,0 \\
$\mathrm{~B}$ & 0,0 & 1,3 \\
\hline
\end{tabular}

Tabela 2.9: Batalha dos sexos.

são simétricos e ambas as estratégias $(F, F)$ e $(B, B)$ são equilíbrios de Nash em estratégias puras. Por outro lado, o perfil $(0,25 B+0,75 F ; 0,75 B+0,25 F)$ é ainda um equilíbrio de Nash em estratégias mistas. Vemos claramente que o equilíbrio de Nash pode não ser único.

No exemplo anterior há três equilíbrios distintos. O primeiro deles atribui o pagamento dado pelo par ordenado $(3,1)$, o segundo atribui o pagamento $(1,3)$ e o terceiro atribui o pagamento $(0,75 ; 0,75)$. Notemos que, dentre os primeiros dois equilíbrios, não há como melhorarmos o pagamento de um dos jogadores sem que, necessariamente, pioremos o pagamento do outro. Se, no caso 
do primeiro equilíbrio, melhorarmos o pagamento do jogador 2 de 1 para 3 , mudando para o segundo equilíbrio, então, necessariamente, pioramos o pagamento do jogador 1 , que cai de 3 para 1 . O mesmo ocorre se mudarmos do segundo equilíbrio para o primeiro, em detrimento do jogador 1, neste caso. Entretanto, o terceiro equilíbrio, em estratégias mistas, não apresenta esta característica. Existe um perfil de estratégias que oferece um pagamento melhor para todos os jogadores e caracteriza um equilíbrio do jogo. Neste sentido dizemos que o equilíbrio $(0,25 B+0,75 F ; 0,75 B+0,25 F)$ é ineficiente (de Pareto). Vejamos:

Definição 11 (Equilíbrios de Nash Pareto-Eficientes) ${ }^{7}$ Considere o jogo

$$
\Gamma^{n}=\left\langle I,\left(\Delta\left(C_{i}\right)\right)_{i \in I},\left(u_{i}\right)_{i \in I}\right\rangle .
$$

Dizemos que $\sigma^{*}$ é um equilíbrio de Nash eficiente (de Pareto) para $\Gamma^{n}$ se não houver nenhum outro equilíbrio de Nash $\sigma^{\prime}$ para $\Gamma^{n}$ tal que

$$
u_{i}\left(\sigma^{\prime}\right) \geq u_{i}\left(\sigma^{*}\right)
$$

para todo $i \in I$.

Se um equilíbrio não for eficiente (de Pareto) ele será dito ineficiente (de Pareto).

Vimos que, no dilema do prisioneiro, nenhuma estratégia pura é estritamente dominada, o que impossibilitou a solução do jogo através do jogo residual. Por outro lado, pudemos apreciar a elegância e o significado do equilíbrio de Nash. Uma pergunta natural neste contexto é sobre a existência dos EN em jogos na forma estratégica.

Vejamos o exemplo da tabela 2.4. Neste caso notamos um jogo que não possui equilíbrios de Nash em estratégias puras. Entretanto, possui como equilíbrios em estratégias mistas o perfil $\left(\frac{2}{3} R A+\frac{1}{3} R R ; \frac{1}{3} P+\frac{2}{3} E\right)$.

Este fato - a inexistência de um equilíbrio de Nash em estratégias puras, mas a sua existência em estratégias mistas - não é um fato isolado, mas sim decorre do teorema que ficou conhecido como Teorema do Equilíbrio de Nash e que afirma que

Teorema 1 (Existência de equilíbrios de Nash) Seja $\Gamma^{n}=\left\langle I,\left(\Delta\left(C_{i}\right)\right)_{i \in I},\left(u_{i}\right)_{i \in I}\right\rangle$ um jogo finito na forma estratégica. Então, existe pelo menos um equilíbrio de Nash $\sigma^{E N} \in \prod_{i \in I} \Delta\left(C_{i}\right)$ para $\Gamma^{n}$.

A demonstração deste resultado fará uso de um teorema de ponto fixo bastante conhecido da Análise, o teorema do ponto fixo de $\mathrm{Kakutani}^{8}$, que generaliza em dimensão finita para o contexto

\footnotetext{
${ }^{7}$ Após Vilfredo Pareto (1848-1923), economista, cientista político e sociólogo italiano. Contribuiu extensivamente para o desenvolvimento de uma Teoria da Escolha em Economia e com o estabelecimento da tradição do Equilíbrio Geral de inspiração da Mecânica Racional. Argumentava pela distinção entre a Economia Pura (caráter técnico) da Economia Aplicada (de caráter social). É sua a frase que diz que o indivíduo pode desaparecer. Não precisamos mais dele para determinar o equilíbrio econômico. Tomada isoladamente pelos críticos, foi o argumento do ataque às idéias de Pareto quando da crítica à Economia Marginalista.

${ }^{8}$ John Nash, ao final de seu trabalho de 1950, no qual demonstra existência do equilíbrio que leva seu nome, faz uma nota de agradecimento ao matemático americano David Gale (1921-2008). Gale foi professor emérito do
} 
de correspondências o resultado do teorema do ponto fixo de Brouwer para funções [36]. Pelo caráter técnico da demonstração, e, pelo propósito deste capítulo, enunciaremos o teorema de Kakutani aqui e exporemos sua demonstração nos apêndices do trabalho. Antes de prosseguirmos, vejamos algumas definições envolvidas neste teorema.

Definição 12 (Ponto fixo) Sejam X um subconjunto de algum espaço Euclidiano m-dimensional $e$

$$
\mathcal{F}: X \longrightarrow 2^{X}
$$

uma correspondência. Então, $x \in X$ será dito um ponto fixo de $\mathcal{F}$ se,

$$
x \in \mathcal{F}(x) .
$$

Definição 13 (Gráfico fechado) Sejam X subconjunto de algum espaço Euclidiano m-dimensional $e$

$$
\mathcal{F}: X \longrightarrow 2^{X}
$$

uma correspondência. Então, diremos que $\mathcal{F}$ tem o gráfico fechado se o conjunto

$$
\mathcal{G}_{\mathcal{F}}=\{(x, y) \mid y \in \mathcal{F}(x)\}
$$

for um subconjunto fechado de $X \times X$ na topologia do produto.

Teorema 2 (Teorema do ponto fixo de Kakutani) Seja $S$ um sub-conjunto convexo, compacto de um espaço Euclidiano n-dimensional. Seja $\mathcal{F}: S \longrightarrow 2^{S}$ uma correspondência de gráfico fechado, tal que para todo $s \in S, \mathcal{F}(s)$ é um subconjunto convexo e não vazio de $S$. Então, deverá existir $\bar{s} \in S$ tal que

$$
\bar{s} \in \mathcal{F}(\bar{s})
$$

Demonstrar a existência de um ponto de equilíbrio de Nash é equivalente, como discutimos, a demonstrar a existência de um ponto fixo para a correspondência de melhor resposta do jogo. Assim, a estratégia de demonstração será, ao tomarmos um jogo $\Gamma^{n}$ finito qualquer, verificarmos que o conjunto $\prod_{i \in I} \Delta\left(C_{i}\right)$, contido em $\mathbb{R}^{n}$, para algum $n \in \mathbb{N}$ apropriado, é não vazio, convexo e compacto. Então deveremos verificar que $\mathcal{B}$, sua correspondência de melhor resposta, possui um gráfico fechado e, para qualquer $\sigma \in \prod_{i \in I} \Delta\left(C_{i}\right)$ temos que $\mathcal{B}(\sigma)$ é um conjunto não vazio e convexo de $\prod_{i \in I} \Delta\left(C_{i}\right)$.

Demonstração do Teorema de Nash. Inicialmente veremos que o conjunto $\Delta=\prod_{i \in I} \Delta\left(C_{i}\right)$ é não vazio, convexo, fechado, e limitado.

departamento de Matemática da Universidade de Berkeley e obteve seu doutorado em Princeton em $1949 . \quad$ Por ocasião de um compromisso externo a Princeton, Alfred Tucker, orientador de Nash e de Gale, incumbiu este último de supervisionar o primeiro enquanto de sua ausência. Em uma destas conversas, David Gale sugeriu a Nash que utilizasse o teorema de Kakutani para fechar seu argumento de existência. Gale foi eleito fellow da Econometric Society em 1965 e contribuiu de maneira ímpar em diversas áreas da Economia Matemática e da Teoria dos Jogos. 
Considere o jogador $i \in I$ e seja $K_{i}=\left|C_{i}\right|$ o número de estratégias puras de $i$. Em primeiro lugar, observamos que $\Delta\left(C_{i}\right)$ é um subconjunto do $\mathbb{R}^{K_{i}}$ cujos pontos são números entre 0 e 1 e cuja soma é exatamente 1 . Assim, vemos que $\Delta\left(C_{i}\right)$ é o simplex do $\mathbb{R}^{K_{i}}$. Concluímos que $\Delta \subset \mathbb{R}^{\prod_{i \in I} K_{i}}$ é o simplex de $\mathbb{R}^{\prod_{i \in I} K_{i}}$.

Se $C_{i}$ for vazio para algum $i$ então não há jogo. Supondo que $C_{i}$ é não vazio, segue da discussão acima que $\Delta$ é não vazio, fechado, limitado e convexo.

Por outro lado, da definição da utilidade von Neumann-Morgenstern, a correspondência de melhor resposta $\mathcal{B}$ aplicada a qualquer $\sigma \in \prod_{i \in I} \Delta\left(C_{i}\right)$ é um subconjunto convexo de $\prod_{i \in I} \Delta\left(C_{i}\right)$.

Para ver que $\mathcal{B}(\sigma) \neq \emptyset$ para todo $\sigma$, basta notar que para todo $i \in I$ a função utilidade esperada von Neumann-Morgenstern $u_{i}$ é contínua sobre o compacto $\Delta\left(C_{i}\right)$, o que implica que o conjunto

$$
\mathcal{R}_{i}\left(\sigma_{-i}\right)=\left\{c_{i} \in C_{i} \mid c_{i} \in \arg \max _{d_{i}^{j} \in C_{i}} u_{i}\left(\sigma_{-i}, d_{i}\right)\right\}
$$

é não-vazio.

Assim, ao menos a distribuição de probabilidade que atribui probabilidade 1 a algum elemento de $\mathcal{R}_{i}\left(\sigma_{-i}\right)$ está em $\mathcal{B}_{i}\left(\sigma_{-i}\right)$. Resta-nos mostrar que para todo $\sigma \in \Delta, \mathcal{B}(\sigma)$ tem o gráfico fechado. Este argumento novamente utilizará a continuidade da utilidade esperada.

Considere uma seqüência de pontos em $\Delta$, digamos, $\left(\sigma^{k}\right)_{k \in \mathbb{N}}$, com

$$
\sigma^{k}=\left(\sigma_{1}^{k}, \ldots, \sigma_{n}^{k}\right)
$$

onde $n$ é o número de jogadores. Seja ainda uma seqüência $\left(\tau^{k}\right)_{k \in \mathbb{N}} \operatorname{com} \tau^{k} \in \mathcal{B}\left(\sigma^{n}\right)$. Temos que mostrar que, se $\sigma^{k} \stackrel{k \rightarrow \infty}{\rightarrow} \bar{\sigma}$ e $\tau^{k} \stackrel{k \rightarrow \infty}{\rightarrow} \bar{\tau}$ então

$$
\bar{\tau} \in \mathcal{B}(\bar{\sigma})
$$

Da hipótese de que $\tau^{k} \in \mathcal{B}\left(\sigma^{k}\right)$ para todo $k \in \mathbb{N}$ segue que

$$
u_{i}\left(\sigma_{-i}^{k}, \tau_{i}^{k}\right) \geq u_{i}\left(\sigma_{-i}^{k}, \xi_{i}\right)
$$

para todo $\xi_{i} \in \Delta\left(C_{i}\right)$. Da continuidade da utilidade esperada, segue que

$$
u_{i}\left(\bar{\sigma}_{-i}, \bar{\tau}_{i}\right) \geq u_{i}\left(\bar{\sigma}_{-i}, \xi_{i}\right)
$$

para todo $\xi_{i} \in \Delta\left(C_{i}\right)$. Assim conclui-se que

$$
\overline{\tau_{i}} \in \mathcal{B}_{i}(\bar{\sigma})
$$

Segue que

$$
\bar{\tau} \in \mathcal{B}(\bar{\sigma})
$$


e, portanto, o gráfico da aplicação $\mathcal{B}$ é fechado.

Do teorema do ponto fixo de Kakutani, segue que existe pelo menos um perfil de estratégias $\sigma^{*} \in \Delta$ tal que $\sigma^{*} \in \mathcal{B}\left(\sigma^{*}\right)$, o que implica na existência do equilíbrio de Nash.

Desta maneira, nesta seção, expusemos a noção de equilíbrio de Nash e verificamos que um jogo na forma normal, finito, sempre possui tal equilíbrio em estratégias mistas.

O conceito de equilíbrio de Nash tem aplicações bastante importantes em Teoria Econômica. Por exemplo, mencionamos o trabalho de Arrow e Debreu [8] que utiliza a existência do equilíbrio de Nash para estabelecer a existência do equilíbrio econômico geral. Além disso, seu uso em teoria dos leilões é amplamente estudado no desenho de mecanismos eficientes.

Vimos nesta seção que um equilíbrio de Nash ineficiente (de Pareto) pode ocorrer. E observamos que não é razoável esperarmos que jogadores racionais e inteligentes joguem tais equilíbrios. Com o intuito de abordar quais as formas de equilíbrio que esperamos ser jogadas, Selten e Harsanyi, Myerson e Kreps e Wilson ( [43], [19] e [18]) formularam aqueles conceitos que ficaram conhecidos na literatura como refinamentos dos equilíbrios de Nash.

Nas próximas seções vamos discutir algumas noções de refinamentos do equilíbrio, ou seja, tentativas de impor maiores restrições à racionalidade dos jogadores, no sentido de obtermos, dentre os equilíbrio de Nash, alguns que sejam mais racionais - em algum sentido - do que os outros.

\subsection{O equilíbrio de Nash e a forma extensiva}

Vimos na seção anterior um importante conceito de solução, o equilíbrio de Nash, e demonstramos sua existência. Nesta seção discutiremos a noção de equilíbrio de Nash para jogos na forma extensiva. Em seguida será exposta a noção de equilíbrio sequencial. Então, apresentaremos o conceito de equilíbrio perfeito e será estabelecida a existência de cada um destes refinamentos.

Uma vez que podemos randomizar um jogo na forma normal através de estratégias mistas, uma pergunta natural é como poderíamos randomizar um jogo na forma extensiva. A pergunta tem sentido uma vez que a forma extensiva pode ser modelada como um jogo na forma estratégica ou um jogo multi-agente.

A idéia de randomização na forma extensiva dá origem a uma importante distinção: entre perfil de estratégias mistas e perfil de estratégias comportamentais. ${ }^{9}$

Recordemos-nos de que $C_{i}$ é o conjunto de estratégias puras de um indivíduo ao passo em que $A_{s}$ é o conjunto de todas as ações possíveis do jogador $i$ que controla o conjunto de informações $s$. Assim sendo, temos:

Definição 14 Considere um jogo $\Gamma^{e}$ na forma extensiva. Um perfil de estratégias mistas é um elemento $\sigma \in \prod_{i \in I} \Delta\left(C_{i}\right)$, ou seja, uma estratégia mista da representação de $\Gamma^{e}$ na forma normal. Já um perfil de estratégias comportamentais é um elemento $\varsigma \in \prod_{s \in S^{*}} \Delta\left(A_{s}\right)$, ou seja, uma estratégia mista da representação multi-agente de $\Gamma^{e}$.

\footnotetext{
${ }^{9} \mathrm{O}$ termo estratégias comportamentais é a tradução que escolhemos para behavioral strategy, uma vez que o autor não encontrou na literatura em português uma tradução alternativa.
} 
Apesar de serem de fato diferentes, e de apresentarem implicações distintas sobre um jogo ${ }^{10}$, ambas as estratégias mistas e comportamentais podem ser relacionadas. Para vermos isto, consideramos a noção de compatibilidade.

Recordamos ao leitor que $P(x \mid c)$ é a probabilidade de atingir-se o nó $x$ uma vez que os jogadores estão seguindo o perfil de estratégias puras $c \in \prod_{i \in I} C_{i}$.

Definição 15 Considere um jogador $i \in I$ fixo. Então, dizemos que $s \in S_{i}$ e $c_{i} \in C_{i}$ são compativeis se existir $c_{-i} \in C_{-i}$ tal que

$$
\sum_{x \in Y_{s}} P(x \mid c)>0
$$

Denotaremos por $C_{*}(s)$ o conjunto de todas as estratégias em $C_{i}$ que são compativeis com $s$.

Definição 16 Considere um jogador $i \in I$ fixo. Então, dizemos que $s \in S_{i}$ e $\sigma_{i} \in C_{i}$ são equivalentes se existir $c_{i} \in C_{i}^{*}$ tal que $\sigma_{i}\left(c_{i}\right)>0$.

Para qualquer $s \in S_{i}$ e $a_{s} \in A_{s}$, denotaremos por $C_{i}^{* *}\left(a_{s}, s\right)$ o conjunto de todas as estratégias puras que são compatíveis com $s$ e que, mais ainda, no conjunto de informação $s$ prescrevem o movimento, ou ação, $a_{s}$. Em símbolos:

$$
C_{i}^{* *} \doteq\left\{c_{i} \in C_{i}^{*}(s) \mid c_{i}(s)=a_{s}\right\}
$$

Agora, vejamos como um perfil de estratégias mistas e um perfil de estratégias comportamentais podem ser relacionados de maneira natural:

Definição 17 Uma estratégia comportamental $\varsigma_{i}$ para o jogador $i$ é uma representação comportamental da estratégia mista $\sigma_{i}$ se, para todo $s \in S_{i}$ e para todo $a_{s} \in A_{s}$, temos que

$$
\varsigma_{i}\left(a_{s}\right) \cdot\left(\sum_{e_{i} \in C_{i}^{*}(s)} \sigma_{i}\left(e_{i}\right)\right)=\sum_{c_{i} \in C_{i}^{* *}\left(a_{s}, s\right)} \sigma_{i}\left(c_{i}\right) .
$$

Definição 18 Uma estratégia mista $\sigma_{i}$ é a representação mista da estratégia comportamental $\varsigma_{i}$ se

$$
\sigma_{i}\left(c_{i}\right)=\prod_{s \in S_{i}} \varsigma_{i}\left(c_{i}(s)\right)
$$

De posse destas definições, podemos responder à pergunta sobre qual solução é adequada para o caso de um jogo na forma extensiva - se o equilíbrio de Nash da sua representação normal ou multi-agente.

Definição 19 Considere um jogo $\Gamma^{e}$ na forma extensiva. Então, $\varsigma \in \prod_{s \in S^{*}} \Delta\left(A_{s}\right)$ é um equilíbrio de Nash para $\Gamma^{e}$ se for um equilíbrio de Nash da sua forma multi-agente, cuja representação mista é um equilíbrio de Nash da forma normal de $\Gamma^{e}$.

\footnotetext{
${ }^{10} \operatorname{Ver}[43]$.
} 
Assim como vimos para o caso da forma normal, veremos um teorema de existência para o equilíbrio de Nash dado como na definição acima. Vejamos uma proposição auxiliar e então o teorema de existência.

Proposição 1 Seja $\Gamma^{e}$ um jogo na forma extensiva e consideremos $\sigma$ um equilíbrio da forma

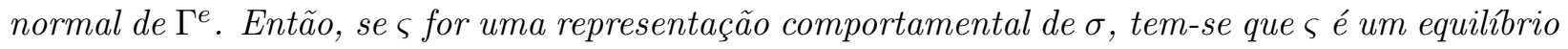
da representação multi-agente de $\Gamma^{e}$.

Demonstração. Consideremos um equilíbrio $\sigma$ da forma normal de $\Gamma^{e}$ e uma sua representação comportamental $\varsigma$.

Suponhamos por absurdo que $\varsigma$ não seja um equilíbrio de Nash para $\Gamma^{e}$ na forma multi-agente. Então, deverá existir algum jogador $i \in I$, algum conjunto de informação $r \in S_{i}$ e alguma estratégia $a_{r}$ em $\Delta\left(A_{r}\right)$ tal que vale para a utilidade multi-agente

$$
v_{i}: \prod_{r \in S^{*}} A_{r} \longrightarrow \mathbb{R}
$$

a seguinte desigualdade

$$
v_{i}\left(\varsigma_{-i, r}, a_{r}\right)>v_{i}(\varsigma)
$$

Assim, se considerarmos a representação mista de $\varsigma_{i}$, substituindo $\varsigma_{i, r}$ por $a_{r}$, e a denotarmos por $\lambda_{i}$, temos que

$$
u_{i}\left(\sigma_{-i}, \lambda_{i}\right)=v_{i}\left(\varsigma_{i, r}, a_{r}\right)>v_{i}(\varsigma)=u_{i}(\sigma)
$$

o que é um absurdo posto que $\sigma$ é um equilíbrio de Nash da forma normal.

Por contradição, o resultado segue.

Teorema 3 Seja $\Gamma^{e}$ um jogo na forma extensiva com recordação perfeita. Então, o conjunto de equilíbrios de Nash em estratégias comportamentais é não vazio.

Demonstração. Do teorema de existência de Nash, vemos que dado $\Gamma^{e}$ sua representação normal terá sempre um equilíbrio de Nash. Da proposição anterior, vemos que este tal equilíbrio de Nash tem em sua representação comportamental um equilíbrio de Nash comportamental do jogo.

\subsection{A forma extensiva e a racionalidade sequencial}

Nesta seção veremos como caracterizar o equilíbrio (de Nash) sequencial de um jogo na forma extensiva. Para tanto, vamos discutir alguns elementos que desempenharão papel crucial em nossa argumentação.

Em um jogo na forma extensiva, o conjunto $L$ de rótulos do jogo, especifica uma probabilidade para cada evento que segue um nó da natureza. Já, se considerarmos um nó de decisão, teremos que uma estratégia comportamental especificará tal probabilidade. Suponha os nós $x$ e $y$. Então, $\bar{P}(y \mid \varsigma, x)$ denotará o produto de todas as probabilidades (do acaso ou de cada jogador) no caminho entre $x$ e $y$. Claramente, $\bar{P}(y \mid \varsigma, x)$ poderá ser nula em dois casos: se não houver nenhum trajeto ligando $x$ a $y$ ou se $\varsigma$ especificar tal probabilidade. 
Definição 20 Sejam $\Omega$ o conjunto dos nós terminais de $\Gamma^{e}$ e $u_{i}(y)$ o pagamento do jogador $i$ quando o nó terminal $y \in \Omega$ é alcançado. Então,

$$
U_{i}(\varsigma \mid x)=\sum_{y \in \Omega} \bar{P}(y \mid \varsigma, x) u_{i}(y)
$$

é o pagamento esperado do jogador $i$ caso o jogo comece em $x$ e todos os jogadores sigam a prescrição dada por $\varsigma$.

A questão apreciada pelo equilíbrio sequencial pode ser entendida como um mecanismo para determinarmos, dentre todos os perfis de estratégias comportamentais, quais são aqueles que, uma vez que um jogador $i$ alcance um determinado conjunto de informação $s \in S_{i}$, ele de fato terá interesse em jogar de acordo com aquele perfil. Em outros termos, quais seriam os perfis de estratégias comportamentais para os quais os jogadores teriam razões para acreditar ${ }^{11}$ que tal perfil é sua melhor ação a fim de maximizar seus interesses.

Para entendermos com precisão esta noção, consideremos um conjunto de informação $s \in S_{i}$ para um jogador $i \in I$ e seu conjunto de nós em $s$ dado por $Y_{s}$. Considere então que, uma vez que $i$ saiba que está em algum nó $x \in Y_{s}$ este jogador tem uma crença, que denotaremos por $\pi_{i, s}(x)$ e que significará a probabilidade do jogo ter alcançado o nó $x \in Y_{s}$ dado que o jogo alcançou o conjunto de informação $s$. Precisamente, temos que

Definição 21 Uma distribuição de probabilidade de crenças para o jogador $i \in I$, em seu conjunto de informação $s \in S_{i}, \pi_{i, s}(\cdot) \in \Delta\left(Y_{s}\right)$, é a probabilidade condicional que $i$ atribui a cada nó $x \in Y_{s}$ dado que o jogo alcançou o conjunto de informação $s$.

O vetor $\pi=\left(\pi_{1, s_{1}^{1}}, \ldots, \pi_{1, s_{1}^{k_{1}}}, \ldots, \pi_{n, s_{n}^{1}}, \ldots, \pi_{n, s_{n}^{k_{n}}}\right) \in \prod_{s \in S^{*}} \Delta\left(Y_{s}\right)$ é chamado vetor de distribuição de probabilidade de crenças do jogo e atribui, para cada jogador $i \in I$ e para cada conjunto de informação em $s \in S_{i}$ uma tal probabilidade condicional.

A idéia de racionalidade sequencial depende das crenças que cada jogador possui sobre cada um de seus conjunto de informação. Deste modo,

Definição 22 Dado um vetor de distribuição de probabilidade de crenças $\pi \in \prod_{s \in S^{*}} \Delta\left(Y_{s}\right)$, um perfil de estratégias comportamentais $\varsigma \in \prod_{s \in S^{*}} \Delta\left(A_{s}\right)$ é dito seqüencialmente racional para o jogador $i$ em seu conjunto de informação s se, e somente se,

$$
\varsigma_{i, s} \in \arg \max _{\rho_{s} \in \Delta\left(A_{s}\right)} \sum_{x \in Y_{s}} \pi_{i, s}(x) U_{i}\left(\varsigma_{-i, s}, \rho_{s} \mid x\right)
$$

\footnotetext{
${ }^{11}$ Aqui, o termo acreditar refere-se àquilo que vamos ver adiante como um sistema de crenças do jogador, ou expectativas, formadas a partir de uma distribuição de probabilidade sobre os estados da natureza. A literatura tem recentemente endereçado este tema a partir das noções de incerteza e ambigüidade, baseando-se para tanto em diferentes axiomatizações da teoria de probabilidades, como no caso da probabilidade subjetiva. Ver, por exemplo, [48], [20] e [28].
} 
Em palavras, podemos dizer que um perfil $\varsigma$ é seqüencialmente racional com relação a um vetor de crenças $\pi$ se, para cada jogador em cada conjunto de informação, $\varsigma$ for um ponto fixo da aplicação de melhor resposta quando o pagamento esperado de cada jogador depender das probabilidades condicionais dadas por $\pi$.

Definição 23 A probabilidade a priori de um nó y, sob o perfil de estratégias comportamentais $\varsigma$ é dado por

$$
\bar{P}(y \mid \varsigma)=\bar{P}\left(y \mid \varsigma, x^{0}\right)
$$

onde $x^{0}$ é a raiz da árvore do jogo.

Definição 24 Um vetor de distribuição de probabilidade de crenças $\pi \in \prod_{s \in S^{*}} \Delta\left(Y_{s}\right)$ é dito fracamente consistente com um perfil de estratégias comportamentais $\varsigma \in \prod_{s \in S^{*}} \Delta\left(A_{s}\right)$ se, para todo $i \in I$, para todo $s \in S_{i}$ e para todo $x \in Y_{s}$ temos que

$$
\pi_{i, s}(x)=\frac{\bar{P}(x \mid \varsigma)}{\sum_{y \in Y_{s}} \bar{P}(y \mid \varsigma)} .
$$

Chamamos a atenção do leitor para o fato de que, a forma como os jogadores compõem suas crenças, ou como atribuem distribuições de probabilidade a cada possível nó do jogo não é discutida neste trabalho e nem naqueles que introduziram a noção de equilíbrio sequencial.

É bem verdade que autores como Halpern [28], sugerem que o simples estabelecimento de um vetor de crenças pode não ser bastante elucidativo do ponto de vista da racionalidade. Uma forma de criticar esta idéia é perguntar sobre como cada jogador atribui probabilidade a um evento sobre o qual tem incerteza ou possui alguma ambigüidade ou simplesmente não pode atribuir um número entre 0 e 1 com alguma segurança. Esta falha abre largo espaço para as recentes contribuições da teoria da escolha que contemplam a noção de incerteza knightiana ou a axiomática de AnscombeAumann, ou mesmo o uso da teoria das capacidades e probabilidades não arquimedianas, cujos impactos sobre os refinamentos do equilíbrio ainda se encontram em muitos aspectos desconhecidos. Estudos nesta direção podem oferecer sentido mais preciso ao entendimento da racionalidade sequencial.

Feita esta observação, e de posse das definições acima, estamos prontos para a definição de equilíbrio sequencial fraco.

Definição 25 Dado um jogo na forma extensiva $\Gamma^{e}$, dizemos que um par $(\varsigma, \pi) \in \prod_{s \in S^{*}} \Delta\left(A_{s}\right) \times$ $\prod_{s \in S^{*}} \Delta\left(Y_{s}\right)$ é um equilíbrio sequencial fraco para $\Gamma^{e}$ se $\varsigma$ é seqüencialmente racional com respeito a $\pi$ e $\pi$ é fracamente consistente com $\varsigma$.

Em outros termos, o par $(\varsigma, \pi)$ é um equilíbrio sequencial fraco se as equações 2.2 e 2.3 estão satisfeitas.

Mais do que isso, dizemos que um perfil de estratégias comportamentais $\varsigma$ é um equilíbrio sequencial fraco ou, com mais cuidado, que tal perfil pode ser estendido a um equilíbrio sequen- 
cial fraco, quando for possível encontrarmos um vetor de crenças $\pi$ tal que $(\varsigma, \pi)$ caracteriza um equilíbrio sequencial fraco.

Observe que a condição 2.2 requer que as probabilidades de que um particular conjunto de informação seja atingido seja diferente de zero. Ou seja, ऽ deve, no contexto da racionalidade sequencial fraca, atribuir probabilidade positiva a todo conjunto de informação. É claro que isto pode não ser verdadeiro.

Assim sendo, é necessário construirmos uma noção mais rigorosa de equilíbrio sequencial, para o qual estratégias comportamentais que atribuem probabilidade nula a um determinado conjunto de informação também possam ser consideradas.

O conjunto $\Delta^{0}\left(A_{s}\right)$ é o conjunto das distribuições que atribuem probabilidade estritamente positiva para cada elemento $a_{s} \in A_{s}$. Assim, temos a definição:

Definição 26 Dizemos que um vetor de distribuição de probabilidade de crenças $\pi \in \prod_{\in S^{*}} \Delta\left(Y_{s}\right)$ é completamente (ou fortemente) consistente com um perfil de estratégias comportamentais $\varsigma \in$ $\prod_{s \in S^{*}} \Delta\left(A_{s}\right)$ para o jogo $\Gamma^{e}$ se existir uma seqüência $\left(\varsigma^{n}\right)_{n \in \mathbb{N}}$ tal que

$$
\begin{array}{r}
\varsigma^{k} \in \prod_{s \in S^{*}} \Delta^{0}\left(A_{s}\right) \quad \forall \quad k \in \mathbb{N} \\
\varsigma_{i, s}\left(a_{s}\right)=\lim _{k \rightarrow \infty} \varsigma_{i, s}^{k} \quad \forall \quad i \in I, \forall s \in S_{i}, a_{s} \in A_{s} \\
\pi_{s}(x)=\lim _{k \rightarrow \infty} \frac{\bar{P}\left(x \mid \varsigma^{k}\right)}{\sum_{y \in Y_{s}} \bar{P}\left(y \mid \varsigma^{k}\right)} \quad \forall s \in S^{*}, \forall x \in Y_{s} .
\end{array}
$$

Em palavras, diremos que um vetor de crenças $\pi$ será completamente (ou fortemente) consistente com um perfil de estratégias comportamentais $\varsigma$ se houver perfis de estratégias comportamentais arbitrariamente próximos de $\varsigma$ que atribuem probabilidade estritamente positiva para cada movimento e o vetor de crenças que satisfaz a regra de Bayes para cada um destes perfis converge para $\pi$.

De modo análogo ao que foi feito anteriormente, vamos definir agora o equilíbrio sequencial completo, ou forte.

Definição 27 Considere um jogo na forma extensiva $\Gamma^{e}$. Diremos que um par

$$
(\varsigma, \pi) \in \prod_{s \in S^{*}} \Delta\left(A_{s}\right) \times \prod_{s \in S^{*}} \Delta\left(Y_{s}\right)
$$

é um equilíbrio sequencial completo (ou forte) se $\pi$ for completamente consistente com $\varsigma$ e, com respeito ao vetor de crenças $\pi$, $\varsigma$ é um perfil de estratégias comportamentais seqüencialmente racional para todo jogador $i \in I$ e para todo $s \in S_{i}$.

Novamente, um perfil de estratégias comportamentais $\varsigma$ será dito um cenário de equilíbrio sequencial forte, ou que poderá ser estendido a um equilíbrio sequencial forte, se houver $\pi \in$ $\prod_{s \in S^{*}} \Delta\left(Y_{s}\right)$ tal que $(\varsigma, \pi)$ é um equilíbrio sequencial forte. 
O leitor deve se recordar que na subseção anterior discutimos a noção e a existência de equilíbrios de Nash para jogos na forma extensiva. Agora, foi discutida a noção de equilíbrio sequencial (tanto fraco como completo). Questões imediatas são, primeiro, sobre a existência de um equilíbrio sequencial. Em segundo lugar, sobre como este se relaciona com o equilíbrio de Nash para jogos na forma extensiva.

$\mathrm{Na}$ verdade, gostaríamos que o equilíbrio sequencial fosse capaz de restringir, dentre aqueles resultados que são equilíbrios de Nash, os que satisfazem os critérios de racionalidade sequencial. Em outras palavras, se denotarmos por $\mathcal{E}_{\text {Nash }}^{\Gamma^{e}}$ o conjunto dos equilíbrios de Nash de um jogo $\Gamma^{e} \mathrm{e}$ por $\mathcal{E}_{\mathcal{S}}^{\Gamma^{e}}$ o conjunto de seus equilíbrios seqüenciais, gostaríamos que fosse

$$
\mathcal{E}_{\mathcal{S}}^{\Gamma^{e}} \subset \mathcal{E}_{\text {Nash }}^{\Gamma^{e}}
$$

De fato esta inclusão é a afirmação do teorema que será demonstrado a seguir.

Teorema 4 Se o par $(\varsigma, \pi) \in \prod_{s \in S^{*}} \Delta\left(A_{s}\right) \times \prod_{s \in S^{*}} \Delta\left(Y_{s}\right)$ é um equilíbrio sequencial forte do jogo na forma extensiva $\Gamma^{e}$ com recordação perfeita, então $\varsigma$ é um equilíbrio de estratégias comportamentais para $\Gamma^{e}$.

Demonstração. Novamente, nossa demonstração será feita por redução ao absurdo. Suponhamos um par $(\varsigma, \pi)$ que é um equilíbrio sequencial forte de $\Gamma^{e}$ e que $\varsigma$ não seja um perfil de equilíbrio em estratégias comportamentais.

Então, existe um jogador $i$ e um perfil comportamental $\theta_{i}$ tal que, de acordo com a notação da forma multi-agente, temos

$$
v_{i}\left(\varsigma_{-i}, \theta_{i}\right)>v_{i}(\varsigma)
$$

Tomemos $\theta_{i}$ tal que a desigualdade acima seja válida e, mais ainda, para qualquer estratégia comportamental $\xi_{i}$ tal que

$$
v_{i}\left(\varsigma_{-i}, \xi_{i}\right)>v_{i}(\varsigma)
$$

tenhamos que

$$
\left|\left\{s \in S_{i} \mid \xi_{i, s} \neq \varsigma_{i, s}\right\}\right| \geq\left|\left\{s \in S_{i} \mid \theta_{i, s} \neq \varsigma_{i, s}\right\}\right| \text {. }
$$

Seja $\bar{s} \in S_{i}$ um conjunto de informações para o jogador $i$ tal que $\theta_{i, \bar{s}} \neq \varsigma_{i, \bar{s}}$ e, para todo $s \in S_{i}$ tal que os nós em $Y_{s}$ são consecutivos aos nós em $Y_{\bar{s}}$ tem-se que $\theta_{i, s}=\varsigma_{i, s}$. Defina ainda $\bar{\theta}_{i, r}$ como a estratégia comportamental que difere de $\theta_{i, r}$ apenas para $r=\bar{s}$.

Em particular, $\bar{\theta}_{i, s}=\theta_{i, s}=\varsigma_{i, s}$ para todo conjunto de informação $s$ que segue o conjunto de nós $Y_{\bar{s}}$. Isto implica que, para todo $y \in Y_{\bar{s}}$ temos que

$$
U_{i}\left(\varsigma_{-i}, \bar{\theta}_{i} \mid y\right)=U_{i}\left(\varsigma_{-i, \bar{s}}, \bar{\theta}_{i, \bar{s}} \mid y\right)
$$

Como $\bar{\theta}$ e $\theta$ diferem apenas no conjunto de informação $\bar{s}$, e, novamente, pela hipótese de recordação perfeita, temos que, para todo $x \in Y_{\bar{s}}$ e para todo $x \in X(\bar{s})$, i.e., o conjunto de to- 
dos os nós terminais que não seguem $\bar{s}$,

$$
\bar{P}\left(x \mid \varsigma_{-i}, \bar{\theta}_{i}\right)=\bar{P}\left(x \mid \varsigma_{-i}, \theta_{i}\right) .
$$

Neste caso, a hipótese de recordação perfeita é crucial para garantir que o conjunto de informação $\bar{s}$ pode ocorrer no máximo uma vez em um dado trajeto da raiz do jogo até um nó qualquer e que cada jogador sabe disso e recorda-se disso perfeitamente em cada instante do jogo.

Assim, temos que

$$
v_{i}\left(\varsigma_{-i}, \bar{\theta}_{i}\right)=\sum_{y \in Y_{\bar{s}}} \bar{P}\left(y \mid \varsigma_{-i}, \theta_{i}\right) U_{i}\left(\varsigma_{i, \bar{s}}, \bar{\theta}_{i, \bar{s}} \mid y\right)+\sum_{x \in X(\bar{s})} \bar{P}\left(x \mid \varsigma_{-i}, \theta_{i}\right) u_{i}(x) .
$$

Considere agora o trajeto da raiz do jogo até algum $x \in Y_{\bar{s}}$. É razoável supor que os conjuntos de informação que ocorrem neste trajeto, bem como as ações sob controle do jogador $i$ são os mesmos para qualquer $x \in Y_{\bar{s}}$.

Assim, dada qualquer estratégia comportamental de $i, \tau_{i}$, por exemplo, então

$$
\frac{\bar{P}(y \mid \tau)}{\sum_{z \in Y_{\bar{s}}} \bar{P}(z \mid \tau)}
$$

não deverá depender de $\tau_{i}$ - uma vez que a escolha por algum destes nós não é prescrita por $\tau_{i}$.

Isto implica em

$$
\bar{P}\left(y \mid \varsigma_{-i}, \theta_{i}\right)=\pi_{i, \bar{s}}(y)\left(\sum_{z \in Y_{\bar{s}}} \bar{P}\left(z \mid \varsigma_{-i}, \theta_{i}\right)\right),
$$

para todo $y \in Y_{\bar{s}}$.

Substituindo a expressão acima em uma anterior para $v_{i}$ temos que

$$
v_{i}\left(\varsigma_{-i}, \bar{\theta}_{i}\right)=\left(\sum_{y \in Y_{\bar{s}}} \pi_{i, \bar{s}}(y) U_{i}\left(\varsigma_{-i, \bar{s}}, \bar{\theta}_{i, \bar{s}} \mid y\right)\right)\left(\sum_{z \in Y_{\bar{s}}} \bar{P}\left(z \mid \varsigma_{-i}, \theta_{i}\right)\right)+\sum_{x \in X(\bar{s})} \bar{P}\left(x \mid \varsigma_{-i}, \theta_{i}\right) u_{i}(x) .
$$

Para maximizar a expressão de $v_{i}$ acima com relação a sua segunda coordenada, sabemos que, da definição do equilíbrio sequencial completo, devemos ter que $\bar{\theta}_{i, \bar{s}}=\varsigma_{i, \bar{s}}$. Entretanto, isto implica que

$$
v_{i}\left(\varsigma_{-i}, \bar{\theta}_{i}\right)>v_{i}(\varsigma)
$$

com

$$
\left|\left\{s \in S_{i} \mid \bar{\theta}_{i, s} \neq \varsigma_{i, s}\right\}<\right|\left\{s \in S_{i} \mid \theta_{i, s} \neq \varsigma_{i, s}\right\}
$$

o que implica em um absurdo, uma vez que tal $\bar{\theta}$ não poderia existir.

Assim, $\varsigma$ é de fato um equilíbrio em estratégias comportamentais.

A questão da existência de equilíbrios seqüenciais será abordada na próxima subseção, quando 
da discussão de equilíbrios perfeitos de um jogo na forma normal. Será possível vermos que todo jogo na forma normal possui um equilíbrio perfeito e que, todo equilíbrio perfeito pode ser estendido a um equilíbrio sequencial. Deixamos esta discussão para a próxima seção para inseri-la em um contexto mais natural.

\subsection{A forma normal e o equilíbrio perfeito}

Considere um jogo na forma normal $\Gamma^{n}$. Suponha que, no exato momento de fazer uma escolha, um particular jogador tem uma ausência de racionalidade ou se esquece de suas preferências sobre os possíveis resultados do jogo. Suponha ainda outra situação: todas as estratégias mistas de um jogador se encontram num painel e para escolher uma delas o jogador deve apertar um botão correspondente. Considere que o jogador, ao escolher, treme sua mão e, por engano, aperta um botão diferente daquele que desejava.

Questões deste tipo foram abordadas por Reinhard Selten durante a década de 1970 e a noção de equilíbrio perfeito para jogos na forma normal traduz a preocupação com conceitos de solução que selecionam pontos com alguma estabilidade local. Esta estabilidade traduz-se através da idéia de que um equilíbrio perfeito é um ponto em $\prod_{i \in I} \Delta\left(C_{i}\right)$ para o qual uma vizinhança arbitrariamente pequena é ainda uma coleção de equilíbrios. Vejamos a definição formal de equilíbrio perfeito.

Definição 28 Considere o jogo na forma normal $\Gamma^{n}=\left\langle I,\left(C_{i}\right)_{i \in I},\left(u_{i}\right)_{i \in I}\right\rangle$, finito. Um perfil de estratégias mistas $\sigma \in \prod_{i \in I} \Delta\left(C_{i}\right)$ será dito um equilíbrio perfeito para $\Gamma^{n}$ se houver uma seqüência $\left(\sigma^{k}\right)_{k \in \mathbb{N}}$ de elementos de $\prod_{i \in I} \Delta\left(C_{i}\right)$ tal que:

$$
\begin{array}{r}
\sigma^{k} \in \prod_{i \in I} \Delta^{0}\left(C_{i}\right) \quad \forall k \in \mathbb{N} \\
\sigma_{i}\left(c_{i}\right)=\lim _{k \rightarrow \infty} \sigma_{i}^{k}\left(c_{i}\right) \quad \forall i \in \forall c_{i} \in C_{i} \\
\sigma_{i} \in \arg \max _{\tau_{i} \in \Delta\left(C_{i}\right)} u_{i}\left(\sigma_{-i}^{k}, \tau_{i}\right) \quad \forall i \in I .
\end{array}
$$

Da última condição da definição anterior temos que

$$
u_{i}\left(\sigma_{-i}^{k}, \sigma_{i}\right) \geq u_{i}\left(\sigma_{-i}^{k}, \tau_{i}\right)
$$

para todo $i \in I$, para todo $k \in \mathbb{N}$ e para todo $\tau_{i} \in \Delta\left(C_{i}\right)$. Assim sendo, e da continuidade da utilidade esperada von Neumann-Morgenstern, temos que, tomando limites,

$$
u_{i}\left(\sigma_{-i}, \sigma_{i}\right) \geq u_{i}\left(\sigma_{-i}, \tau_{i}\right)
$$

para todo $i \in I$, e para todo $\tau \in \Delta\left(C_{i}\right)$. Assim, se denotarmos por $\mathcal{E}_{\text {Nash }}^{\Gamma^{n}}$ o conjunto dos equilíbrios de Nash de $\Gamma^{n}$ e por $\mathcal{E}_{\mathcal{P}}^{\Gamma^{n}}$ seu conjunto de equilíbrios perfeitos, é clara a seguinte proposição.

Proposição 2 Seja $\Gamma^{n}$ um jogo na forma normal. Então, todo equilíbrio perfeito de $\Gamma^{n}$ é um equilíbrio de Nash para $\Gamma^{n}$ Ou seja,

$$
\mathcal{E}_{\mathcal{P}}^{\Gamma^{n}} \subset \mathcal{E}_{\text {Nash }}^{\Gamma^{n}}
$$


O leitor - nem tão - atento, deve ter notado a semelhança entre a definição de equilíbrio perfeito para um jogo normal e a definição de equilíbrio sequencial completo para um jogo na forma extensiva: em ambos os conceitos de solução o equilíbrio é perturbado e a partir de uma sua vizinhança são geradas ou crenças (equilíbrio sequencial) ou são testadas condições de otimalidade, ou de ponto fixo da correspondência de melhor resposta.

Esta semelhança é de fato um elemento que nos permite relacionar ambos os conceitos de solução. Observando que a condições para o equilíbrio perfeito são sutilmente mais restritivas obtemos o seguinte

Teorema 5 Considere um jogo $\Gamma^{e}$ na forma extensiva e seja $\sigma$ um equilíbrio de sua representação multi-agente. Então, existe um vetor de distribuição de probabilidade de crenças $\pi$ tal que $(\sigma, \pi)$ é um equilibrio sequencial completo de $\Gamma^{e}$.

Demonstração. Seja $\left(\sigma^{k}\right)_{k \in \mathbb{N}}$ uma seqüência em $\prod_{r \in S^{*}} \Delta^{0}\left(A_{r}\right)$ que satisfaz as condições de equilíbrio perfeito 2.4-2.6 para a forma multi-agente de $\Gamma^{e}$ e considere ainda $\left(\pi^{k}(y)\right)_{k \in \mathbb{N}}$ dada por

$$
\pi^{k}(y)=\frac{\bar{P}\left(y \mid \sigma^{k}\right)}{\sum_{z \in Y_{s}} \bar{P}\left(z \mid \sigma^{k}\right)},
$$

para cada $y \in Y_{s}$, para cada $s \in S_{i}$ e para cada $i \in I$.

Por conta do espaço em que a seqüência $\sigma^{k}$ é definida, cada probabilidade na razão anterior é estritamente positiva, e, assim, o quociente está bem definido.

Tomando limites e fazendo $\pi_{s}(y)=\lim _{k \rightarrow \infty} \pi_{s}^{k}$, temos que $\pi$ é um vetor de crenças completamente consistente com o perfil $\sigma$.

Vamos verificar agora que $\sigma$ é seqüencialmente racional, dado o vetor $\pi$.

Utilizando a notação da forma multi-agente, temos que

$$
v_{s}\left(\sigma_{-i, s}^{k}, \rho_{i, s}\right)=\sum_{y \in Y_{s}} \bar{P}\left(y \mid \sigma_{-i, s}^{k}, \rho_{i, s}\right) U_{i}\left(\sigma_{-i, s}^{k}, \rho_{i, s} \mid y\right)+\sum_{x \in X(s)} \bar{P}\left(x \mid \sigma_{-i, s}^{k}, \rho_{i, s}\right) u_{i}(x) .
$$

Observe que, a probabilidade do jogo alcançar algum nó $y \in Y_{s}$ depende de probabilidades atribuídas a movimentos anteriores a $y$ - novamente é a hipótese de recordação perfeita que nos permite afirmar que este fato é verdade e que os jogadores têm ciência dele. Assim, $\bar{P}\left(y \mid \sigma_{-i, s}^{k}, \rho_{i, s}\right)$ não depende de $\rho_{i, s}$, posto que esta prescrição não interfere na probabilidade de $y$ ser alcançado. Assim, segue que

$$
\bar{P}\left(y \mid \sigma_{-i, s}^{k}, \rho_{i, s}\right)=\bar{P}\left(y \mid \sigma^{k}\right) .
$$

Substituindo na expressão anterior para $v_{s}$ segue que

$$
v_{s}\left(\sigma_{-i, s}^{k}, \rho_{i, s}\right)=\sum_{y \in Y_{s}} \bar{P}\left(y \mid \sigma^{k}\right) U_{i}\left(\sigma_{-i, s}^{k}, \rho_{i, s} \mid y\right)+\sum_{x \in X(s)} \bar{P}\left(\left.x\right|^{k}\right) u_{i}(x)=
$$




$$
=\left(\sum_{y \in Y_{s}} \pi_{s}^{k}(y) U_{i}\left(\sigma_{-i, s}^{k}, \rho_{i, s} \mid y\right)\right)\left(\sum_{z \in Y_{s}} \bar{P}\left(z \mid \sigma^{k}\right)\right)+\sum_{x \in X(s)} \bar{P}\left(x \mid \sigma^{k}\right) u_{i}(x) .
$$

Note que, se considerarmos os termos que dependem de $\rho$, então $v_{s}$ pode ser escrita como uma expressão afim, dada por

$$
v_{s}\left(\sigma_{-i, s}^{k}, \rho_{i, s}\right)=A \cdot\left(\sum_{y \in Y_{s}} \pi_{s}^{k}(y) U_{i}\left(\sigma_{-i, s}^{k}, \rho_{i, s} \mid y\right)\right)+B .
$$

O fato de que $\sigma$ é um equilíbrio perfeito cujas condições são satisfeitas por $\left(\sigma^{k}\right)_{k \in \mathbb{N}}$ implica que

$$
\sigma_{s} \in \arg \max _{\rho_{s} \in \Delta\left(A_{s}\right)} v_{s}\left(\sigma_{-i, s}^{k}, \rho_{i, s}\right)
$$

Estas duas observações implicam que

$$
\sigma_{s} \in \arg \max _{\rho_{s} \in \Delta\left(A_{s}\right)}\left(\sum_{y \in Y_{s}} \pi_{s}^{k}(y) U_{i}\left(\sigma_{-i, s}^{k}, \rho_{i, s} \mid y\right)\right) .
$$

Para verificarmos que $\sigma$ é seqüencialmente racional, basta utilizarmos do fato de que o gráfico da correspondência de melhor resposta do jogo é fechado. Assim, tomando o limite na expressão anterior, segue

$$
\sigma_{s} \in_{\rho_{s} \in \Delta\left(A_{s}\right)}\left(\sum_{y \in Y_{s}} \pi_{s}(y) U_{i}\left(\sigma_{-i, s}, \rho_{i, s} \mid y\right)\right),
$$

exatamente a condição que garante o resultado.

Os dois próximos teoremas garantem a existência de equilíbrios perfeitos e seqüenciais.

Teorema 6 Para qualquer jogo na forma normal, finito, existe pelo menos um equilíbrio perfeito.

Demonstração. Considere um jogo na forma normal finito dado por $\Gamma^{n}=\left\langle I,\left(C_{i}\right)_{i \in I},\left(u_{i}\right)_{i \in I}\right\rangle$. Considere ainda a estratégia mista $\lambda_{i}$, dita uniforme, i.e., para cada $c_{i} \in C_{i}$,

$$
\lambda_{i}\left(c_{i}\right)=\frac{1}{\left|C_{i}\right|} .
$$

É claro que $\lambda_{i} \in \Delta^{0}\left(C_{i}\right)$.

Por fim, para cada $k \in \mathbb{N}$ seja a aplicação

$$
\delta^{k}: \prod_{i \in I} \Delta\left(C_{i}\right) \longrightarrow \prod_{i \in I} \Delta^{0}\left(C_{i}\right)
$$


dada por

$$
\delta^{k}(\sigma)=\tau \quad \Longleftrightarrow \forall i \in I, \forall c_{i} \in C_{i}, \tau_{i}\left(c_{i}\right)=\left(1-\left(\frac{1}{k}\right)\right) \sigma_{i}\left(c_{i}\right)+\frac{1}{k} \lambda_{i}\left(c_{i}\right)
$$

Analogamente, tomamos

$$
\delta^{k}(\sigma)=\tau \Longleftrightarrow \forall i \in I, \tau_{i}=\left(1-\left(\frac{1}{k}\right)\right) c_{i}+\frac{1}{k} \lambda_{i}\left(c_{i}\right) .
$$

Construindo uma função de pagamento $\hat{u}: \prod_{i \in I} C_{i} \longrightarrow \mathbb{R}$ por

$$
\hat{u}_{i}^{k}(c)=u_{i}\left(\delta^{k}(c)\right)
$$

obtemos o jogo finito na forma estratégica dado por

$$
\hat{\Gamma}^{k}=\left\langle I,\left(C_{i}\right)_{i \in I},\left(\hat{u}_{i}^{k}\right)_{i \in I}\right\rangle
$$

O jogo em 2.7 satisfaz às hipóteses do teorema de existência de equilíbrios de Nash e, portanto, possui um EN, digamos, $\bar{\sigma}^{k}$.

Se entendermos $\left(\bar{\sigma}^{k}\right)_{k \in \mathbb{N}}$ como uma seqüência de elementos de um conjunto compacto - $\prod_{i \in I} \Delta\left(C_{i}\right)$ - é claro que esta possui uma subseqüência convergente. Suponhamos, em benefício da notação, que a própria seqüência $\bar{\sigma}^{k}$ convirja para um ponto, digamos, $\sigma^{*}$.

Observe que, como $C_{i}$ é finito, também será finito o conjunto de suas partes. Assim, passando novamente a uma subseqüência, podemos supor que os pontos de $C_{i}$ aos quais é atribuída probabilidade nula em $\bar{\sigma}^{k}$ são os mesmos para todo $i \in I$ e para todo $k \in \mathbb{N}$.

Defina $\hat{\sigma}^{k}=\delta^{k}\left(\bar{\sigma}^{k}\right)$. Então,

$$
\hat{\sigma}^{k} \in \prod_{i \in I} \Delta^{0}\left(C_{i}\right)
$$

para todo $k$ natural. Da definição de $\delta$, segue que $\delta \bar{\sigma}^{k}=\bar{\sigma}^{k}$. Então,

$$
\lim _{k \longrightarrow \infty} \hat{\sigma}^{k}=\lim _{k \longrightarrow \infty} \bar{\sigma}^{k}=\sigma^{*} .
$$

Para cada jogador $i$ e para cada natural $k$, se $c_{i} \in C_{i}$ não é a melhor resposta de $i$ dado que os demais jogadores procedem como prescrito por $\hat{\sigma}_{-i}^{k}$, então $c_{i}$ deverá receber probabilidade nula de acordo com $\hat{\sigma}_{i}^{k}$. Assim, $\sigma_{i}^{*}\left(c_{i}\right)=0$. Obtém-se assim que

$$
\sigma_{i}^{*} \in \arg \max _{\tau_{i} \in \Delta\left(C_{i}\right)} u_{i}\left(\hat{\sigma}_{-i}^{k}, \tau_{i}\right)
$$

para todo $k \in \mathbb{R}$.

Assim, $\sigma^{*}$ satisfaz às condições que caracterizam o equilíbrio perfeito. 
Teorema 7 Para qualquer jogo na forma extensiva, finito, existe pelo menos um equilíbrio sequencial (forte).

Demonstração. Imediata a partir dos teoremas 5 e 6 anteriores. 


\section{Capítulo 3}

\section{Teoria dos Jogos Diferenciais}

Neste capítulo apresentaremos os elementos básicos da teoria dos jogos diferenciais e formularemos o problema cujas técnicas de solução serão delineadas nos capítulos posteriores.

\subsection{Exemplos introdutórios}

Com o intuito de tornar a apresentação da formulação matemática do jogo diferencial mais clara, alguns exemplos de jogos serão apresentados.

Exemplo 4 [Jogo de perseguição [32]] Considere dois indivíduos, um predador P e uma presa E, cada um deles se movendo com velocidade constante $v_{P}$ e $v_{E}$ numa arena retangular finita - que

pode ser entendida como um aberto $\Omega \subset \mathbb{R}^{2}$. Suponha que a posição de cada um deles na arena seja dada por coordenadas da forma $P=\left(P_{1}, P_{2}\right)$ e $E=\left(E_{1}, E_{2}\right)$. Por fim, suponha que o predador $P$ decide, a cada instante do tempo, sobre o ângulo $\omega_{P}$ que seu vetor velocidade forma com o eixo horizontal, enquanto que a presa $E$ decide, também em cada instante, sobe o ângulo $\omega_{E}$ de seu vetor velocidade com relação ao eixo horizontal. Neste sentido, a dinâmica que rege o jogo diferencial descrito acima é dada pelas equações:

$$
\begin{aligned}
& \dot{P}_{1}=v_{P} \cos \omega_{P} \\
& \dot{P}_{2}=v_{P} \sin \omega_{P}
\end{aligned}
$$

$e$

$$
\begin{aligned}
& \dot{E}_{1}=v_{E} \cos \omega_{E} \\
& \dot{E}_{2}=v_{E} \sin \omega_{E} .
\end{aligned}
$$

O predador deseja escolher a direção do vetor velocidade de modo a alcançar a presa no menor intervalo de tempo possivel, i.e., o predador escolhe $\omega_{P}$ de modo a minimizar o tempo de captura. Por outro lado, a presa deverá escolher $\omega_{E}$ tendo em vista impedir, se possivel, ou, ao menos, maximizar o tempo de captura. Uma forma de avaliarmos a performance de cada um dos jogadores é estabelecermos uma função de performance, de medida ou, mais geralmente, uma função de pagamento. Suponhamos que o jogo tenha sido iniciado em um instante $t_{0}$ do tempo. Então, a 
função de pagamento pode ser definida como

$$
\mathcal{P}_{t_{0}}(t)=t-t_{0}
$$

a qual, claramente, o predador tentará minimizar, ao passo em que a presa tentará maximizar.

No exemplo 4 acima, foi mencionada a noção de captura. De fato, este conceito em um jogo de perseguição e evasão pode ser bastante vago. Podemos definir captura por coincidência, ou seja, o predador terá capturado a presa caso $P=E$. É também possível que a noção de captura seja baseada em proximidade, ou seja, dado $\varepsilon>0$, dizemos que o predador captura a presa caso $\|P-E\|<\varepsilon$.

Exemplo 5 [Predador-presa, [32]] Considere um predador $P$ e uma presa E se movendo sobre um aberto limitado $\Omega \subset \mathbb{R}^{2}$. A posição da presa é descrita pelo ponto $E=\left(E_{1}, E_{2}\right)$, sua velocidade é constante igual a $v_{E}$ e ela escolhe o ângulo que seu vetor velocidade forma com o eixo horizontal, $\omega_{E}$. Por outro lado, o predador, denotado por $P$, tem sua posição descrita por duas variáveis: sua posição no plano, dada por $P=\left(P_{1}, P_{2}\right)$ e o ângulo que seu vetor velocidade forma com o eixo horizontal, $\omega_{P}$. Suponha que o predador escolhe uma fração, entre zero e um, da aceleração máxima que deseja utilizar. Então, dada a aceleração máxima $A$ o predador escolhe $\psi_{1}^{P} \in[0,1]$, o que faz com que sua aceleração seja dada por $A \psi_{1}^{P}$. Uma vez que sua velocidade $v_{P}$ não está mais sob seu controle direto, mas sim depende da sua aceleração, ela também será tomada como uma variável de estado. Suponha ainda que o predador não possa variar sua curvatura arbitrariamente, mas, sim, de forma limitada. Seja $C$ a máxima curvatura possível. Então, o predador deve ainda escolher $\psi_{2}^{P} \in[-1,1]$, a taxa de curvatura máxima que será utilizada. Sua curvatura será dada, então, por $\kappa_{P}$. A dinâmica do jogo pode ser descrita por

$$
\begin{array}{r}
\dot{P}_{1}=v_{P} \cos \omega_{P} \\
\dot{P}_{2}=v_{P} \sin \omega_{P} \\
\dot{\omega}_{P}=v_{P} \kappa_{P} \\
\dot{v}_{P}=A \psi_{1}^{P} \\
\dot{\kappa}_{P}=C \psi_{2}^{P}
\end{array}
$$

$e$

$$
\begin{aligned}
\dot{E}_{1} & =v_{E} \cos \omega_{E} \\
\dot{E}_{2} & =v_{E} \sin \omega_{E}
\end{aligned}
$$

Novamente, podemos propor uma função de pagamento como a do exemplo anterior.

O exame destes exemplos nos permite identificar alguns elementos comuns. Primeiramente, tratam-se de jogos de dois jogadores e simétricos, na medida em que um jogador tem o objetivo 
de maximizar e o seu oponente tem o objetivo de minimizar um dado funcional. Uma vez que os jogadores possuem objetivos antagônicos sobre o funcional de pagamento, trata-se de um jogo não-cooperativo.

Por outro lado, o jogador que maximiza a função pagamento, recebe $\mathcal{P}$, ao passo que o jogador que a minimiza recebe $-\mathcal{P}$. Jogos com esta característica são chamados de jogos de soma-zero uma vez que o pagamento de ambos somam zero.

Além disso, todos eles são regidos por uma dinâmica contínua com relação ao tempo no contexto da qual os jogadores escolhem controles a fim de otimizarem a função pagamento. Estes fatos não são isolados, mas caracterizam uma classe de jogos diferenciais sobre os quais recairá nosso interesse, nominalmente, os jogos diferenciais qualitativos. Passemos à próxima seção para a discussão formal destes objetos.

\subsection{Definições e resultados preliminares}

Ocuparemos-nos nesta seção da discussão do jogo diferencial segundo Isaacs.

Definição 29 [Jogo Diferencial, [32]] Um jogo diferencial é uma 6-upla $\Gamma=\langle I, f, \Omega, \mathcal{U}, \mathcal{V}, \mathcal{P}\rangle$ onde

1. $I=\{1,2\}$ é o conjunto de jogadores,

2. $f \in \mathcal{C}\left(\Omega \times \mathcal{U} \times \mathcal{V} \times \mathbb{R}, \mathbb{R}^{n}\right)$ é a função que engendra a dinâmica do jogo

$$
\dot{x}(t)=f(x(t), u(t), v(t), t),
$$

3. $\mathcal{U}$ e $\mathcal{V}$ são os espaços de controles admissíveis dos jogadores 1 e 2, respectivamente e,

4. $\mathcal{P}$ é a função de pagamento do jogo, sobre a qual os jogadores têm objetivos opostos.

Na definição acima, o aberto $\Omega \in \mathbb{R}^{n}$, para um $n$ apropriado, é o chamado espaço de estados do jogo diferencial, ou seja, o espaço em que varia a trajetória $x(t)$. A função de pagamento de um jogo diferencial $\mathcal{P}$, dependendo da situação que visamos modelar, pode apresentar formulações distintas. Por exemplo, podemos ter funções de pagamento dos tipos:

1. Horizonte temporal infinito

$$
\mathcal{P}(x, u, v) \doteq \int_{0}^{\infty} g(x, u, v) e^{-\lambda} d t
$$

2. Horizonte temporal finito - dada uma função $g \in \mathcal{C}\left(\mathbb{R}^{n}\right)$,

$$
\mathcal{P}(x, u, v) \doteq g(x, u, v)
$$


3. Tempo mínimo com taxa de desconto $s$

$$
\mathcal{P}(x, u, v) \doteq \int_{0}^{t_{x}(u, v)} e^{-s} d s=1-e^{-t_{x}(u, v)}
$$

caso $t_{x}(u, v)<+\infty$. Caso contrário, $\mathcal{P}(x, u, v)=1$.

Uma questão que deve ser colocada é a existência e unicidade de trajetórias ótimas, dado uma condição inicial do espaço de estados e controles arbitrários. Neste sentido, no que diz respeito à existência, utilizamos as bem conhecidas condições de Caratheodory ${ }^{1}$ que garantem a existência de uma trajetória, dado um ponto inicial do espaço de estados e controles quaisquer. Já para analisarmos a existência de trajetórias ótimas, nos valemos das condições necessárias prescritas pelo princípio da programação dinâmica ou pelo princípio do máximo de Pontriaguin. É ainda com base nestas ferramentas que a questão da unicidade é abordada. Para detalhes acerca destes resultados, ver [4] e [37].

Em particular, neste caso, solucionar o jogo significa obtermos os controles ótimos utilizados pelos jogadores em cada instante do tempo. Mais precisamente, suponha que o jogo ocorre em um intervalo de tempo $[0, T]$; então, resolver o jogo consiste na obtenção de uma aplicação que retorna o controle ótimo $\left(u^{*}(t), v^{*}(t)\right)$ dado um instante $t \in[0, T]$ de tempo e um ponto do espaço de estados $x_{0}$ que entendemos como a condição inicial do sistema.

A solução de um jogo diferencial, entretanto, não é uma questão trivial e passa pela determinação de uma chamada função de valor do jogo - sua existência e suas propriedades. Assim sendo, antes de iniciarmos uma discussão sobre a solução do jogo, é necessário discutirmos a função de valor.

Duas abordagens à função de valor do jogo são familiares à teoria do jogos diferenciais. A primeira delas se baseia na chamada condição de Isaacs [32] e parte de uma noção bastante intuitiva: um jogo terá uma função de valor quando o problema de otimização do pagamento for independente da ordem, ou seja, quando não importar se o jogador 1 maximiza primeiro ou se o jogador 2 minimiza primeiro. Formalmente, um jogo terá uma função de valor caso tenha uma solução MinMax, ou, se verifique a condição de Isaacs:

$$
\max _{u \in \mathcal{U}} \min _{v \in \mathcal{V}} \mathcal{P}(x, u, v)=\min _{v \in \mathcal{V}} \max _{u \in \mathcal{U}} \mathcal{P}(x, u, v)
$$

Esta abordagem pode ser entendida da seguinte forma: por um lado, o jogador 1 - que quer maximizar a função pagamento - considera que o jogador 2 irá escolher a cada instante o controle que a minimize. Assim, de modo a obter o melhor resultado dentre os piores possíveis, o jogador 1

\footnotetext{
${ }^{1}$ Constantino Caratheodory, ou $K \omega \nu \sigma \tau \alpha \nu \tau \iota \nu o \varsigma K \alpha \rho \alpha \theta \varepsilon o \delta \omega \rho \eta$, foi um matemático de ascendência grega nascido em Berlim a 13 de setembro de 1873. Durante os anos 1902-1904, concluiu seus estudos de doutoramento sob a orientação de Hermann Minkowski na Universidade de Göttingen. É autor de contribuições importantes à teoria das medidas exteriores e da termodinâmica e responsável por uma conjectura acerca de superfícies convexas. É também autor de um pequeno livro sobre aspectos geográficos e históricos do Egito - devido a um projeto ao qual esteve ligado antes de seu doutoramento. Em 2009, a cidade de origem da família de Constantino, Kotomi, na Grécia, abriu as portas do Museu Caratheodory, no qual estão presentes, dentre outras peças, correspondências trocadas com Albert Einstein. Caratheodory morreu em Munique, a 2 de fevereiro de 1950.
} 
maximizará ao invés da função de pagamento, o seu mínimo com relação aos controles utilizados por 2 .

Por outro lado, suponha que o jogador 1 maximize o pagamento tendo em vista diferentes estados da natureza - imaginamos uma situação estocástica cuja distribuição de probabilidade é desconhecida pelo jogador. Então, a solução de MaxMin reflete ainda procurar pelo melhor resultado dentre os piores possíveis, i.e., pode constituir uma primeira abordagem a um problema de otimização estocástica, quando os estados da natureza, cuja distribuição de probabilidade é desconhecida, podem afetar o pagamento do jogador.

Adiante, veremos quais condições asseguram a existência da função de valor de Isaacs.

A segunda abordagem à função de valor do jogo baseia-se na noção de jogo $\delta$-superior e jogo $\delta$-inferior bem como na noção de convergência de suas respectivas funções de valor, e é devida a Friedman [24]. Apresentaremos inicialmente uma discussão acerca da função de valor de Isaacs e, em seguida, abordaremos a versão de Friedman para o problema.

Considerando o jogo definido acima, estudemos agora as condições necessárias à existência do valor do jogo segundo Isaacs. Para tanto, alguns elementos da topologia geral são necessários.

Definição 30 Uma função $f: X \longrightarrow \mathbb{R}$ definida sobre um espaço separado é dita convexa se para quaisquer elementos $x, y \in X \quad e \forall \lambda \in[0,1]$ temos

$$
f[\lambda x+(1-\lambda) y] \leq \lambda f(x)+(1-\lambda) f(y)
$$

Por outro lado, uma função $g: X \longrightarrow \mathbb{R}$ definida sobre um espaço separado é dita côncava se para quaisquer elementos $x, y \in X$ e $\forall \lambda \in[0,1]$ temos

$$
\lambda f(x)+(1-\lambda) f(y) \leq f[\lambda x+(1-\lambda) y]
$$

As figuras 3.1 e 3.2 ilustram estes conceitos.

Definição 31 Sejam $X$ um espaço topológico separado e $f: X \longrightarrow \mathbb{R}$ uma função. Então $f$ será dita semi-contínua inferiormente (SCI) num ponto $x \in X$ se $\forall \varepsilon>0$ existir uma vizinhança aberta de $x$, denotada $V_{x}$, tal que $y \in V_{x}$ implica $f(x)-f(y) \leq \varepsilon$. Como é usual, $f$ é dita semi-contínua inferiormente se for semi-contínua inferiormente em todos os pontos $x \in X$.

De modo análogo, podemos definir uma função semi-contínua superiormente (SCS).

De modo mais simples, a noção de semi-continuidade sugere que em uma direção a função não tem saltos, i.e., uma função semi-contínua inferiormente [superiormente] não dá saltos para baixo [para cima]. Para entendermos de maneira mais clara o que significa a noção de semi-continuidade, vejamos alguns exemplos:

Exemplo 6 1. Vejamos um exemplo de uma aplicação semi-contínua superiormente. Considere a função $f(x)=\lfloor x\rfloor$, a chamada função piso. Esta aplicação retorna, para cada número real $x$, o maior inteiro menor ou igual a $x$. 


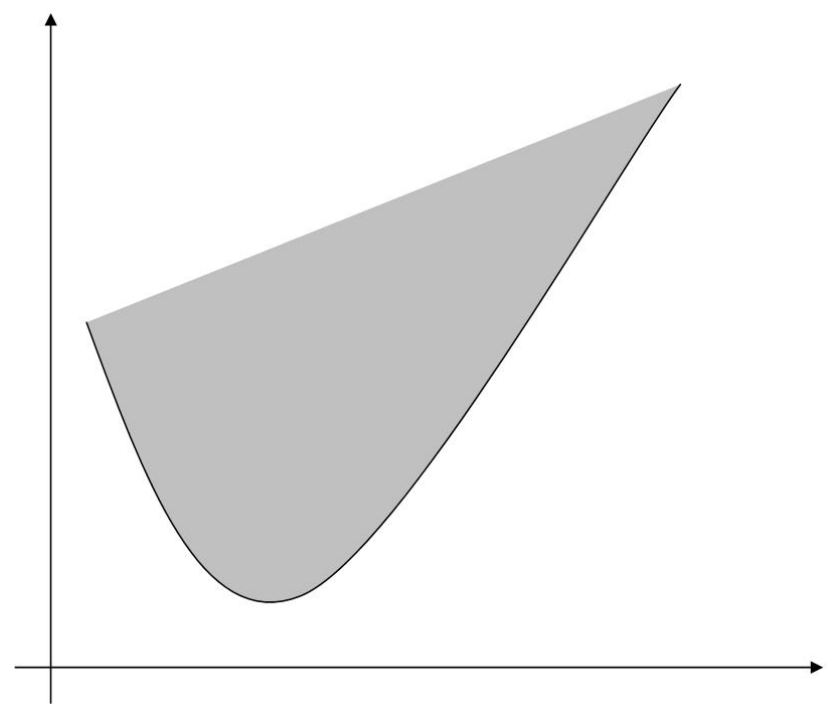

Figura 3.1: Um exemplo de função convexa.

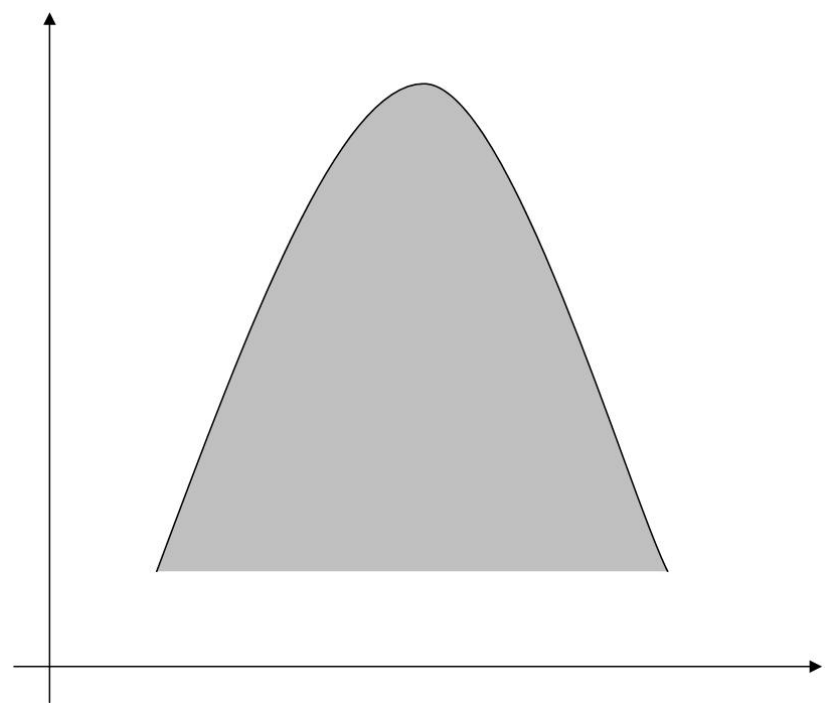

Figura 3.2: Um exemplo de função côncava.

2. Por outro lado, a aplicação teto, $g(x)=\lceil x\rceil$, que retorna o menor inteiro maior ou igual que $x$, é semi-contínua inferiormente. Vemos na figura 3.4 o gráfico da aplicação teto.

Lema 1 Seja $f: X \longrightarrow \mathbb{R}$ uma função semi-contínua inferiormente. Então, para todo $\alpha \in \mathbb{R} o$ conjunto $f^{-1}((\alpha,+\infty)) \subset X$ é aberto. ${ }^{2}$

Demonstração. Tome $\alpha$ fixo e considere $x \in f^{-1}((\alpha,+\infty))$. Como $(\alpha,+\infty)$ é aberto em $\mathbb{R}$ então existe uma vizinhança de $f(x)$, denotada $V_{f(x)}$, tal que $V_{f(x)} \in(\alpha,+\infty)$. Por $f$ ser SCI, para todo $\varepsilon>0$, existe uma vizinhança de $x$, denotada $V_{x}$, tal que se $y \in V_{x}$ então $f(x)-f(y)<\varepsilon$. Deste modo, $y \in f^{-1}((\alpha,+\infty))$ e, portanto, $V_{x} \in f^{-1}((\alpha,+\infty))$, de onde segue o resultado.

\footnotetext{
${ }^{2} \mathrm{O}$ conteúdo deste lema garante que uma função SCI é mensurável.
} 


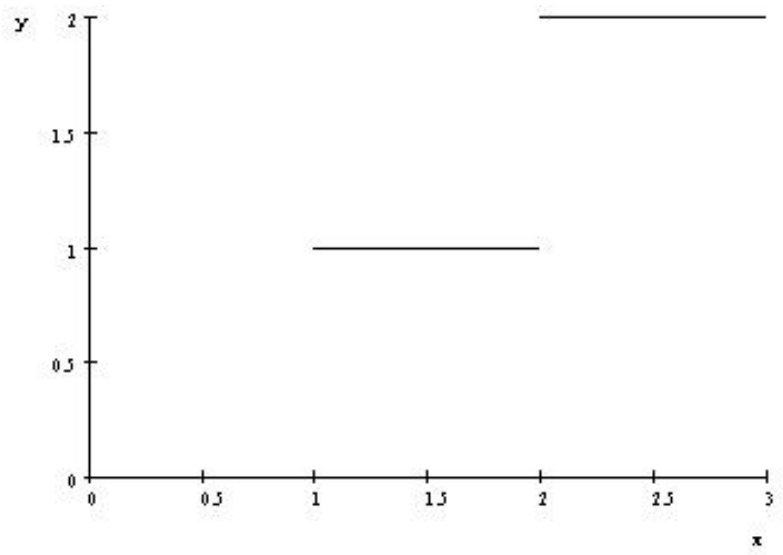

Figura 3.3: Gráfico da aplicação $f(x)=\lfloor x\rfloor$.

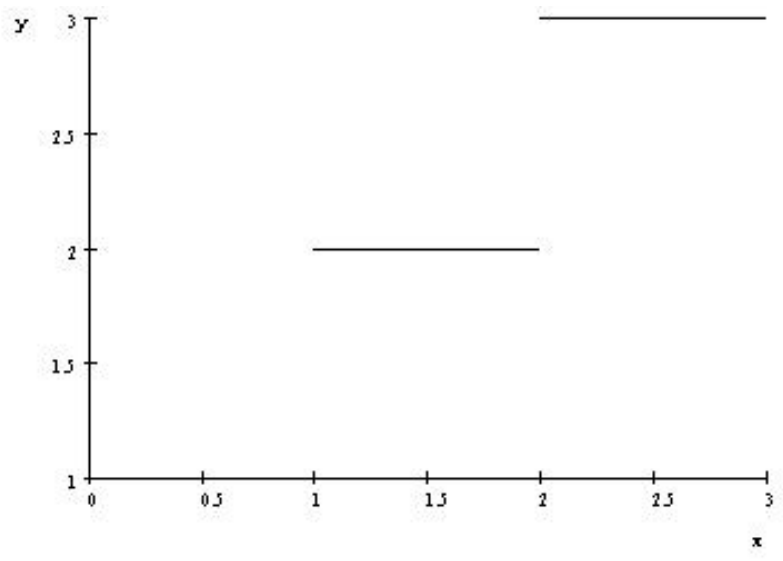

Figura 3.4: Gráfico da aplicação $f(x)=\lceil x\rceil$.

Por passagem ao complementar temos o

Corolário 1 Seja $f: X \longrightarrow \mathbb{R}$ uma função semi-contínua inferiormente. Então, para todo $\alpha \in \mathbb{R}$ o conjunto $f^{-1}([\alpha,+\infty)) \subset X$ é fechado.

Definição 32 Seja X um espaço topológico e $\mathcal{C}$ uma coleção de subconjuntos fechados de X. Então, dizemos que $\mathcal{C}$ atende à condição da interseção finita se para toda sub-coleção finita $C_{n} \subset \mathcal{C} a$ interseção $\bigcap_{C \in C_{n}} C$ é não vazia.

Lema 2 Seja $X$ um espaço separado. Então, $X$ é compacto se, e somente se, toda coleção arbitrária $\mathcal{C}$ de subconjuntos fechados de $X$, que satisfaz a condição da interseção finita, possui interseção não vazia.

Demonstração. Seja $\mathcal{C}$ uma coleção qualquer de subconjuntos fechados de $X$ que satisfaça à condição da interseção finita. Seja agora

$$
\mathcal{A}=\{X \backslash C \mid C \in \mathcal{C}\} .
$$


Claramente, $\mathcal{A}$ é uma coleção de subconjuntos abertos de $X$. A condição da interseção finita implica que se nenhuma subfamília finita de $\mathcal{A}$ cobre $X$ então $\mathcal{A}$ não pode cobrir $X$, de onde segue o resultado.

\subsection{Existência da função de valor de Isaacs}

Consideremos $H, K$ espaços separados, que supomos compactos, ${ }^{3}$ e uma função $f: H \times K \longrightarrow$ $\mathbb{R}$. Tomemos, arbitrariamente, $\bar{h} \in H$ e $\bar{k} \in K$. Claramente

$$
f(\bar{h}, \bar{k}) \leq \max _{k \in K} f(\bar{h}, k)
$$

A desigualdade continua válida caso tomemos o mínimo de ambos os lados, com relação a $h \in H$,

$$
\min _{h \in H} f(h, \bar{k}) \leq \min _{h \in H} \max _{k \in K} f(h, k)
$$

Por fim, como a desigualdade acima é válida para k arbitrário, vem:

$$
\max _{k \in K} \min _{h \in H} f(h, \bar{k}) \leq \min _{h \in H} \max _{k \in K} f(h, k)
$$

Com isso, demonstramos o seguinte

Lema 3 Seja $f: H \times K \rightarrow \mathbb{R}$ uma função a valores reais definida sobre o produto cartesiano de dois espaços separados. Então vale

$$
\max _{k \in K} \min _{h \in H} f(h, k) \leq \min _{h \in H} \max _{k \in K} f(h, k) .
$$

Para garantirmos a condição de Isaacs, basta oferecermos as condições sob as quais

$$
\min _{v \in \mathcal{V}} \max _{u \in \mathcal{U}} \mathcal{P}(u, v, t) \leq \max _{u \in \mathcal{U}} \min _{V \in \mathcal{V}} \mathcal{P}(u, v, t)
$$

Teorema 8 [Teorema do MinMax, Ky Fan, [23]] Sejam $X$ e $Y$ dois espaços topológicos separados, compactos e $f: X \times Y \rightarrow \mathbb{R}$. Suponha que para todo $y \in Y, f(x, y)$ é semi-continua inferiormente em $X$ e, para todo $x \in X, f(x, y)$ é semi-contínua superiormente em $Y$. Então:

1. A igualdade

$$
\min _{x \in X} \max _{y \in Y} f(x, y)=\max _{y \in Y} \min _{x \in X} f(x, y)
$$

vale se e somente se para quaisquer conjuntos finitos $\left\{x_{1}, \ldots, x_{n}\right\} \subset X e\left\{y_{1}, \ldots, y_{m}\right\} \subset Y$ existirem $x^{*} \in X$ e $y^{*} \in Y$ tais que a condição

$$
f\left(x^{*}, y_{j}\right)=f\left(x_{k}, y^{*}\right)
$$

é satisfeita para todo $1 \leq j \leq m, 1 \leq k \leq n$.

\footnotetext{
${ }^{3}$ Do contrário, devemos substituir max e min no que segue por sup e inf, respectivamente.
} 
2. É suficiente para valer a igualdade que $f$ seja convexa em $X$ e côncava em $Y$.

\section{Demonstração.}

1. Demonstraremos inicialmente a necessidade da condição. Considere dois subconjuntos finitos quaisquer $\left\{x_{1}, \ldots, x_{n}\right\} \subset X$ e $\left\{y_{1}, \ldots, y_{m}\right\} \subset Y$. Segue que

$$
\min _{x \in X} \max _{1 \leq j \leq m} f\left(x, y_{j}\right) \leq \min _{x \in X} \max _{y \in Y} f(x, y)
$$

e

$$
\max _{y \in Y} \min _{x \in X} f(x, y) \leq \max _{y \in Y} \min _{1 \leq k \leq n} f\left(x_{k}, y\right) .
$$

Pela hipótese concluímos que

$$
\min _{x \in X} \max _{1 \leq j \leq m} f\left(x, y_{j}\right) \leq \max _{y \in Y} \min _{1 \leq k \leq m} f\left(x_{k}, y\right),
$$

de onde segue a necessidade da condição. Vejamos agora que a condição é suficiente.

De fato ela implica que

$$
\min _{x \in X} \max _{1 \leq j \leq m} f\left(x, y_{j}\right) \leq \max _{y \in Y} \min _{1 \leq k \leq m} f\left(x_{k}, y\right)
$$

é válida para quaisquer $\left\{x_{1}, \ldots, x_{n}\right\} \subset X$ e $\left\{y_{1}, \ldots, y_{m}\right\} \subset Y$. Assim sendo, para todo $\alpha \in \mathbb{R}$,

$$
\min _{x \in X} \max _{1 \leq j \leq m} f\left(x, y_{j}\right) \leq \alpha
$$

$\mathrm{ou}$

$$
\alpha \leq \max _{y \in Y} \min _{1 \leq k \leq m} f\left(x_{k}, y\right)
$$

Definimos então os conjuntos

$$
L(y ; \alpha)=\{x \in X \mid f(x, y) \leq \alpha\}
$$

e

$$
U(x ; \alpha)=\{y \in Y \mid f(x, y) \geq \alpha\} .
$$

Como $f(x, y)$ é semi-contínua superiormente em $Y$ para todo $x \in X$ e semi-contínua inferiormente em $X$ para todo $y \in Y$, ambos os conjuntos são fechados. Como, para todo $\left\{x_{1}, \ldots, x_{n}\right\} \subset X$ e $\left\{y_{1}, \ldots, y_{m}\right\} \subset Y$ ou $L\left(y_{j} ; \alpha\right)$ não é vazio para nenhum $j$ ou $U\left(x_{k} ; \alpha\right)$ não é vazio para nenhum $k$, segue que ao menos uma das intersecções $\bigcap_{k=1}^{n} U\left(x_{k} ; \alpha\right)$ ou $\bigcap_{j=1}^{m} L\left(y_{j} ; \alpha\right)$ é não vazia.

Da compacidade de $X$ e de $Y$ segue que ao menos uma das duas intersecções $\bigcap_{x \in X} U(x, \alpha)$ ou $\bigcap_{y \in Y} L(y ; \alpha)$ é não vazia. Isto implica que $\exists x^{*} \in X$ tal que $f\left(x^{*}, y\right) \leq \alpha$ para todo $y \in Y$ 
ou $\exists y^{*} \in Y$ tal que $f\left(x, y^{*}\right) \geq \alpha$ para todo $x \in X$. Logo,

$$
\min _{x \in X} \max _{y \in Y} f(x, y) \leq \alpha
$$

$\mathrm{Ou}$

$$
\max _{y \in Y} \min _{x \in X} f(x, y) \geq \alpha
$$

o que implica que

$$
\min _{x \in X} \max _{y \in Y} f(x, y) \leq \max _{y \in Y} \min _{x \in X} f(x, y) .
$$

2. Assumiremos agora que $f$ é convexa em $X$ e côncava em $Y$ e tomaremos novamente $\left\{x_{1}, \ldots, x_{n}\right\} \subset$ $X$ e $\left\{y_{1}, \ldots, y_{m}\right\} \subset Y$ subconjuntos quaisquer. Devido ao teorema do MinMax para estratégias mistas de von Neumann [52] existem $\left\{\lambda_{1}^{X}, \ldots, \lambda_{n}^{X}\right\}$ e $\left\{\lambda_{1}^{Y}, \ldots, \lambda_{m}^{Y}\right\}$ tais que $\lambda_{k}^{X} \geq 0$, $\lambda_{j}^{Y} \geq 0$ para todo $1 \leq k \leq n, 1 \leq j \leq m \operatorname{com} \sum_{k=1}^{n} \lambda_{k}^{X}=1$, e $\sum_{j=1}^{m} \lambda_{j}^{Y}=1$, para os quais,

$$
\max _{1 \leq j \leq m} \sum_{k=1}^{n} \lambda_{k}^{X} f\left(x_{k}, y_{j}\right) \leq \min _{1 \leq k \leq n} \sum_{j=1}^{m} \lambda_{j}^{Y} f\left(x_{k}, y_{j}\right) .
$$

Isto implica a existência de ao menos um $x^{*} \in X$ e de ao menos um $y^{*} \in Y$ tais que

$$
f\left(x^{*}, y_{j}\right) \leq \sum_{k=1}^{n} \lambda_{k}^{X} f\left(x_{k}, y_{j}\right)
$$

$\mathrm{e}$

$$
\sum_{j=1}^{m} \lambda_{j}^{Y} f\left(x_{k}, y_{j}\right) \leq f\left(x_{k}, y^{*}\right)
$$

o que implica a condição do item 1 e, por conseguinte, no resultado desejado.

Desta maneira, dado um jogo diferencial como o definido em na seção 3.2, podemos prescrever as condições sob as quais este terá uma função de valor.

Teorema 9 (da existência da função de valor segunda Isaacs) Seja $\Gamma$ um jogo diferencial cuja função de pagamento satisfaz às seguintes condições:

1. Para todo $v \in \mathcal{V}, \mathcal{P}$ é semi-contínua inferiormente em $\mathcal{U}$ e, para todo $u \in \mathcal{U}, \mathcal{P}$ é semicontinua superiormente em $\mathcal{V}$.

2. $\mathcal{P}$ é convexa em $\mathcal{U}$ e côncava em $\mathcal{V}$.

Então a condição de Isaacs está garantida e $\Gamma$ tem uma função de valor.

Demonstração. Segue diretamente do teorema anterior, tomando $\mathcal{P}=f$ e $\mathcal{U}$ e $\mathcal{V}$ no lugar de $X$ e $Y$.

Passemos agora ao tratamento das noções de jogo diferencial e função de valor devidas a Friedman. 


\subsection{A função de valor de Friedman}

A função de valor no sentido de Friedman [24] é definida a partir da noção de jogo $\delta$-superior e $\delta$-inferior, nos quais os dois jogadores escolhem estratégias de forma alternada e um dos jogadores tem a vantagem de iniciar o jogo. Daremos mais adiante um significado para esta vantagem, ao discutirmos o valor de Friedman em sua re-interpretação oferecida por [14], bem como na aplicação da teoria das soluções viscosas a demonstrações de existência para tal função.

Novamente, tomemos um jogo de dois jogadores, soma-zero, com instante terminal finito, dig$\operatorname{amos} T>0$ e consideremos $\delta>0$ tal que

$$
\delta=\frac{T}{n}
$$

para algum $n \in \mathbb{N}$. Isto nos permite dividir o intervalo $[0, T]$ em sub-intervalos da forma

$$
I_{j}=\left\{t \in[0, T] \mid t_{j-1} \leq t \leq t_{j}\right\}
$$

com $j=1, \ldots, n$. Por simplicidade, $t_{0}=0$ e $t_{n}=T$.

Consideremos agora os espaços de controles admissíveis $\mathcal{U}_{j}$ e $\mathcal{V}_{j}$ definidos sobre $I_{j}$ e definamos

$$
\begin{gathered}
\Gamma^{\delta, 1}: \mathcal{V}_{1} \longrightarrow \mathcal{U}_{1} \\
\Gamma^{\delta, 2}: \mathcal{V}_{1} \times \mathcal{U}_{1} \times \mathcal{V}_{2} \longrightarrow \mathcal{U}_{2}
\end{gathered}
$$

e assim por diante, até

$$
\Gamma^{\delta, j}: \mathcal{V}_{1} \times \mathcal{U}_{1} \times \mathcal{V}_{2} \times \mathcal{U}_{2} \times \ldots \times \mathcal{U}_{j-1} \times \mathcal{V}_{j} \longrightarrow \mathcal{U}_{j}
$$

com $j=1, \ldots, n$. Estas aplicações são agrupadas em um vetor, que chamaremos de estratégia $\delta$-superior para o jogador 1 , e que é dado por:

$$
\Gamma^{\delta}=\left(\Gamma^{\delta, 1}, \ldots, \Gamma^{\delta, n}\right)
$$

Analogamente, consideremos as aplicações

$$
\begin{gathered}
\Delta^{\delta, 1}: \mathcal{U}_{1} \longrightarrow \mathcal{V}_{1} \\
\Delta^{\delta, 2}: \mathcal{U}_{1} \times \mathcal{V}_{1} \times \mathcal{U}_{2} \longrightarrow \mathcal{V}_{2}
\end{gathered}
$$

e assim por diante, até obtermos (para $j=1, \ldots, n)$

$$
\Delta^{\delta, j}: \mathcal{U}_{1} \times \mathcal{V}_{1} \times \mathcal{U}_{2} \times \mathcal{V}_{2} \times \ldots \times \mathcal{V}_{j-1} \times \mathcal{U}_{j} \longrightarrow \mathcal{V}_{j}
$$

Como no caso do jogador 1 , definiremos uma estratégia $\delta$-superior para o jogador 2 como sendo o vetor

$$
\Delta^{\delta}=\left(\Delta^{\delta, 1}, \ldots, \Delta^{\delta, n}\right)
$$


Da mesma forma, definiremos estratégias $\delta$-inferiores para ambos os jogadores. Consideremos $2 \leq j \leq n$ e as aplicações

$$
\begin{gathered}
\Gamma_{\delta, 2}: \mathcal{U}_{1} \times \mathcal{V}_{1} \longrightarrow \mathcal{U}_{2} \\
\Gamma_{\delta, 3}: \mathcal{U}_{1} \times \mathcal{V}_{1} \times \mathcal{U}_{2} \times \mathcal{V}_{2} \longrightarrow \mathcal{U}_{3}
\end{gathered}
$$

e assim sucessivamente, até

$$
\Gamma_{\delta, j}: \mathcal{U}_{1} \times \mathcal{V}_{1} \times \mathcal{U}_{2} \times \mathcal{V}_{2} \times \ldots \times \mathcal{U}_{j-1} \times \mathcal{V}_{j-1} \longrightarrow \mathcal{U}_{j}
$$

Tomemos qualquer regra de escolha de pontos em $\mathcal{U}_{1}$ e a denotemos por $\Gamma_{\delta, 1}$. Então uma estratégia $\delta$-inferior para o jogador 1 é o vetor

$$
\Gamma_{\delta}=\left(\Gamma_{\delta, 1}, \ldots, \Gamma_{\delta, n}\right)
$$

Do mesmo modo, devemos definir uma estratégia $\delta$-inferior para o jogador 2 . Novamente, consideramos $2 \leq j \leq n$ e as aplicações

$$
\begin{gathered}
\Delta_{\delta, 2}: \mathcal{V}_{1} \times \mathcal{U}_{1} \longrightarrow \mathcal{V}_{2} \\
\Delta_{\delta, 3}: \mathcal{V}_{1} \times \mathcal{U}_{1} \times \mathcal{V}_{\in} \times \mathcal{U}_{\in} \longrightarrow \mathcal{V}_{\ni}
\end{gathered}
$$

e assim por diante até

$$
\Delta_{\delta, j}: \mathcal{V}_{1} \times \mathcal{U}_{1} \times \mathcal{V}_{2} \times \mathcal{U}_{2} \times \ldots \times \mathcal{V}_{j-1} \times \mathcal{U}_{j-1} \longrightarrow \mathcal{V}_{j}
$$

Usaremos novamente o artifício de definir $\Delta_{\delta, 1}$ como uma regra que toma valores em $\mathcal{V}_{1}$. Naturalmente, definimos uma estratégia $\delta$-inferior para o jogador 2 como sendo a $n$-upla

$$
\Delta_{\delta}=\left(\Delta_{\delta, 1}, \ldots, \Delta_{\delta, n}\right)
$$

Tomemos agora um par - chamado perfil de estratégias $-\left(\Delta_{\delta}, \Gamma^{\delta}\right)$ e coloquemos $\Delta_{\delta, 1}=v_{1}$ e $\Gamma^{\delta, 1}\left(v_{1}\right)=u_{1}$. Definamos ainda

$$
\begin{gathered}
u_{j}=\Gamma^{\delta, j}\left(v_{1}, u_{1}, \ldots, v_{j-1}, u_{j-1}, v_{j}\right) \\
v_{j}=\Delta_{\delta, j}\left(v_{1}, u_{1}, \ldots, v_{j-1}, u_{j-1}\right) .
\end{gathered}
$$

Em geral, uma estratégia superior para o jogador $i$ indica que este deverá jogar no instante $t$ conhecendo a estratégia de seu oponente neste mesmo instante. Já a estratégia inferior é quando o $i$-ésimo jogador escolhe sua estratégia em cada instante sem conhecer a estratégia de seu oponente naquele instante.

Definição 33 O par $\left(u^{\delta}, v_{\delta}\right)$, formado pelas funções cujos componentes são os pontos $u^{\delta, j}$ e $v_{\delta, j}$, respectivamente, é chamado a realização do par $\left(\Gamma^{\delta}, \Delta_{\delta}\right)$. 
Considerando que em cada intervalo da forma $I_{j}$ cada jogador seleciona um controle que será utilizado durante todo o intervalo, então para calcularmos a função de pagamento do jogo, basta avaliarmos esta função na realização do dado perfil de estratégias,

$$
\mathcal{P}\left(u^{\delta}, v_{\delta}\right)
$$

As equações 3.1 e 3.2 sugerem o esquema no qual o jogo evolui. O jogador 2 inicia o jogo escolhendo um controle $v_{1}$ e, dada esta escolha do jogador 2, o jogador 1 escolherá sua melhor resposta, $\Gamma^{\delta, 1}\left(v_{1}\right)$. E em cada $n$-ésima etapa do jogo este arranjo deve se repetir: 1 sempre escolherá seu $n$-ésimo movimento baseado no $n$-ésimo movimento de 2 .

Em certo sentido, neste arranjo, o jogador 1 toma suas decisões com base em toda a história do jogo. Isto não está disponível para 2 no momento de cada movimento. Na verdade, é 2 quem inicia cada etapa de escolhas.

Definição 34 Seja $\mathcal{G}^{\delta}=\left\langle I, f, \mathcal{U}, \mathcal{V}, \mathcal{P}, \Delta_{\delta}, \Gamma^{\delta}\right\rangle$ um jogo como na definição 29, considerando-se agora as estratégias $\delta$-inferior e $\delta$-superior de cada jogador. Então, $\mathcal{G}^{\delta}$ é chamado jogo diferencial $\delta$-superior.

Dado o jogo diferencial $\Gamma$, definido na seção anterior, era possível derivarmos uma função de valor a ele correspondente. O mesmo será realizado no caso de um jogo $\delta$-superior. Para tanto, vamos assumir que o jogador 1 é aquele que quer maximizar a função de pagamento, enquanto o jogador 2 quer minimizá-la.

Suponha que o jogador 2 tenha realizado todos os $n$ movimentos previstos do jogos e 1 tenha realizado apenas os $(n-1)$-ésimos movimentos. Assim, uma vez que o jogador 1 escolherá o último movimento do jogo, é plausível supor que ele deverá escolher um movimento $\Gamma^{\delta, n *}$ tal que

$$
\mathcal{P}\left(\Delta_{\delta, 1}, \Gamma^{\delta, 1}, \ldots, \Delta_{\delta, n}, \Gamma^{\delta, n *}\right) \geq \mathcal{P}\left(\Delta_{\delta, 1}, \Gamma^{\delta, 1}, \ldots, \Delta_{\delta, n}, \Gamma^{\delta, n}\right)
$$

para todo possível $\Gamma^{\delta, n}$. Em outros termos, 1 jogará tendo em vista

$$
\sup _{\Gamma^{\delta, n}} \mathcal{P}\left(\Delta_{\delta, 1}, \Gamma^{\delta, 1}, \ldots, \Delta_{\delta, n}, \Gamma^{\delta, n}\right)
$$

Por outro lado, o jogador 2, sabendo que 1 é racional e tentando maximizar seu pagamento, antecipará esta estratégia procurando minimizar $\mathcal{P}$ em seu último movimento. Isto significa que 2 jogará tendo em vista

$$
\inf _{\Delta_{\delta, n}} \sup _{\Gamma^{\delta, n}} \mathcal{P}\left(\Delta_{\delta, 1}, \Gamma^{\delta, 1}, \ldots, \Delta_{\delta, n}, \Gamma^{\delta, n}\right) .
$$

Repetindo este processo de forma sucessiva, é plausível supormos que, ambos os jogadores escolherão os movimentos que gerem 


$$
\inf _{\Delta_{\delta, 1}} \sup _{\Gamma^{\delta, 1}} \ldots \inf _{\Delta_{\delta, n}} \sup _{\Gamma^{\delta, n}} \mathcal{P}\left(\Delta_{\delta, 1}, \Gamma^{\delta, 1}, \ldots, \Delta_{\delta, n}, \Gamma^{\delta, n}\right)
$$

\section{Definição 35}

$$
V^{\delta} \doteq \inf _{\Delta_{\delta}} \sup _{\Gamma^{\delta}} \mathcal{P}\left(\Delta_{\delta}, \Gamma^{\delta}\right)=\sup _{\Gamma^{\delta}} \inf _{\Delta^{\delta}} \mathcal{P}\left(\Delta_{\delta}, \Gamma^{\delta}\right)
$$

é chamado valor $\delta$-superior do jogo $\mathcal{G}^{\delta}$.

De forma inteiramente análoga, se tomarmos um par da forma $\left(\Gamma_{\delta}, \Delta^{\delta}\right)$, e construirmos os controles $u_{j}$ e $v^{j}$ em cada sub-intervalo da forma $I_{j}$ tais que $u_{1}=\Gamma_{\delta, 1}$ e $v^{1}=\Delta^{\delta, 1}\left(u_{1}\right)$ e

$$
\begin{array}{r}
u_{j}=\Gamma_{\delta, j}\left(u_{1}, v_{1}, \ldots, u_{j-1}, v_{j-1}\right) \\
v^{j}=\Delta^{\delta, j}\left(u_{1}, v_{1}, \ldots, u_{j-1}, v_{j-1}, u_{j}\right) .
\end{array}
$$

Aqui, as funções $u_{\delta}$ e $v^{\delta}$ cujos componentes são dados por $u_{j}$ e $v^{j}$, formam o par chamado de realização de $\left(\Gamma_{\delta}, \Delta^{\delta}\right)$, denotado por $\left(u_{\delta}, v^{\delta}\right)$, e servem de argumento para a função de pagamento $\mathcal{P}$,

$$
\mathcal{P}\left(u_{\delta}, v^{\delta}\right)
$$

Este arranjo motiva a seguinte

Definição 36 Seja $\mathcal{G}_{\delta}=\left\langle I, f, \mathcal{U}, \mathcal{V}, \mathcal{P}, \Delta^{\delta}, \Gamma_{\delta}\right\rangle$ um jogo como na definição 29, considerando-se agora as estratégias $\delta$-inferior e $\delta$-superior de cada jogador. Então, $\mathcal{G}_{\delta}$ é chamado jogo diferencial $\delta$-inferior.

Aplicando a idéia de que o jogador 1 tem por objetivo maximizar o funcional de pagamento, ao passo em que o jogador 2 tem por objetivo minimizá-lo, podemos derivar o análogo do valor $\delta$-superior, a saber

Definição 37 Chamamos valor $\delta$-inferior do jogo $\mathcal{G}_{\delta}$ ao número

$$
V_{\delta} \doteq \sup _{\Gamma_{\delta}} \inf _{\Delta^{\delta}} \mathcal{P}\left(\Gamma_{\delta}, \Delta^{\delta}\right) \doteq \inf _{\Delta^{\delta}} \sup _{\Gamma_{\delta}} \mathcal{P}\left(\Gamma_{\delta}, \Delta^{\delta}\right)
$$

No caso em que as igualdades das definições 35 e 37 são satisfeitas, dizemos que existem os valores $\delta$-superior e $\delta$-inferior para os jogos $\mathcal{G}^{\delta}$ e $\mathcal{G}_{\delta}$.

O Teorema 8 da seção anterior fornece as condições sob as quais isto se verifica. Entretanto, não é capaz de endereçar o comportamento de $V^{\delta}$ ou $V_{\delta}$ quando $\delta$ varia.

A partir do que foi dito até aqui, podemos definir o jogo diferencial segundo Friedman como a dupla $\mathcal{G}=\left(\mathcal{G}_{\delta}, \mathcal{G}^{\delta}\right)$. Na abordagem original de Friedman, este jogo terá um valor caso

$$
\lim _{\delta \longrightarrow 0} V^{\delta}=V^{+}=V^{-}=\lim _{\delta \longrightarrow 0} V_{\delta}
$$


Assim sendo, as propriedades da função de valor, bem como um teorema de existência, serão estabelecidas com base na teoria das soluções viscosas das equações de Hamilton-Jacobi, e, portanto, ficam reservadas para um capítulo posterior.

\subsection{A Solução do Jogo Diferencial e as Equações de HJ}

Após caracterizarmos o jogo diferencial e verificarmos que ele possui de fato uma função de valor, queremos obter os controles, ou estratégias, ótimas de cada jogador de modo a resolver o jogo em questão.

Esta tarefa pode não ser elementar e deve, na verdade, requerer que sejamos capazes de caracterizar a função de valor do jogo de maneira mais precisa. Para tal feito, duas ferramentas serão de grande valor. A primeira delas é a teoria da programação dinâmica, devida a Bellman [3], enquanto a segunda delas é a teoria das soluções viscosas da equação de Hamilton-Jacobi (HJ).

Através do primeiro ferramental, mostraremos que, caso exista e seja de classe $\mathcal{C}^{1}$ sobre seu domínio, a função de valor de um jogo diferencial é a solução clássica da equação de HJ, em particular, de sua variação chamada Hamilton-Jacobi-Isaacs (HJI). ${ }^{4}$

Entretanto, como a literatura bem aponta - veja [46], por exemplo - as aplicações de maior interesse da teoria dos jogos diferenciais esbarra na baixa regularidade da função de valor associada ao jogo. Assim, é necessário resolvermos o jogo de alguma maneira alternativa. Quando a função de valor for, pelo menos, contínua, então utilizamos a teoria das soluções viscosas da equação de HJI. Através desta noção mais fraca de solução, podemos obter a função de valor do jogo e assim sua solução.

O próximo capítulo faz uma exposição do arcabouço teórico da programação dinâmica enquanto que o capítulo 5 se ocupa da teoria das soluções viscosas das equações de HJ.

\footnotetext{
${ }^{4}$ Estas demonstrações serão expostas nos capítulos seguintes.
} 


\section{Capítulo 4}

\section{O Princípio da Programação Dinâmica}

\subsection{Exemplos e considerações preliminares}

Segundo Bellman [3]

An optimal policy has the property that whatever the initial state and initial decisions are, the remaining decisions must constitute an optimal policy with regard to the state resulting from the first decision.

Em outros termos, podemos parafrasear Bellman afirmando que, de acordo com o princípio da programação dinâmica, em qualquer ponto de uma trajetória ótima que se encontrar o estado de uma particular dinâmica, sua trajetória até o ponto terminal ainda é ótima.

Suponha um veículo que deve percorrer o trajeto que liga os pontos $a$ e $b$ e sejam $J_{a x}$ o custo de percorrer o trecho do ponto $a$ até o ponto $x$ e $J_{x b}$ o custo de percorrer o trecho de $x$ até $b$. Se a trajetória ótima para realizar o percurso for de fato $a-x-b$ então, uma vez que o veículo atinja $x$, o princípio da programação dinâmica estabelece que a trajetória para chegar até $b$ é ótima.

A idéia intuitiva de uma demonstração deste princípio é simples: suponha que a trajetória $a-x-b$ seja ótima, i.e, que $J_{a x b}$ é de fato o custo mínimo para se atingir $b$ saindo de $a$. Então, suponha que, ao atingir $x$ o veículo tenha como alternativa seguir para um ponto $y$ e então seguir para $b$, assumindo que o custo de percorrer esta trajetória seja inferior àquele de percorrer $x-b$. Este fato pode ser escrito como $J_{x y b}<J_{x b}$, ou seja, o custo de seguir passando pelo ponto $y$ é inferior ao custo seguindo para $b$.

Isto implicaria que $J_{a x}+J_{x y b}<J_{a x}+J_{x b}=J_{a x b}$, o que seria absurdo em face à otimalidade de $a-x-b .^{1}$

Para tentarmos entender a aplicação do princípio da programação dinâmica, apresentamos um exemplo.

Exemplo 7 Considere um motorista dirigindo em uma cidade representada pela figura 4.1 abaixo cujo objetivo é alcançar o ponto h com o menor consumo de combustivel possível, partindo de $c$. As setas na figura indicam as direções permitidas em cada via e os números associados a elas representam o custo em unidades de combustivel. Neste problema, o estado é representado por

\footnotetext{
${ }^{1}$ Adiante é oferecida uma demonstração formal do princípio da programação dinâmica.
} 
cada cruzamento em que o motorista possa se encontrar (de a a h) e a variável de controle é cada trecho que ele escolhe para percorrer.

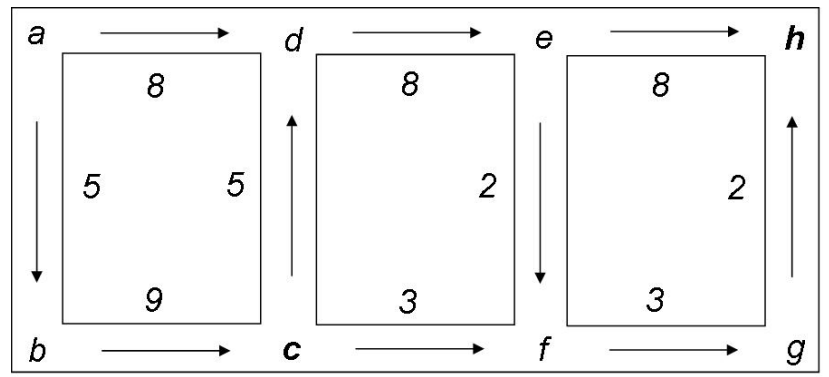

Figura 4.1: Exemplo - Programação Dinâmica.

Uma solução do problema seria calcular o custo de cada uma das possíveis rotas que o motorista poderia seguir e decidir pela menos custosa. Entretanto, esta tarefa parece bastante trabalhosa. Suponhamos que o motorista se encontre no cruzamento c. Suas únicas opções são seguir no sentido $d$ ou no sentido $f$, cujos custos de combustivel são, respectivamente, $J_{c d}$ e $J_{c f}$. Suponhamos ainda que o motorista conheça os custos minimos $J_{d h}^{*}=16$ e $J_{f h}^{*}=5$. Assim sendo, o menor custo total para chegar de c até $h, J_{c h}^{*}$ é dado pelo menor valor entre $C_{c d h}^{*}=J_{c d}+J_{d h}^{*}=5+16 e$ $C_{c f h}^{*}=J_{c f}+J_{f h}^{*}=3+5$.

O algoritmo computacional geral - que considera a hipótese do indivíduo iniciar sua viagem em qualquer ponto - pode ser sintetizado em duas equações. A primeira delas é uma implicação do princípio da otimalidade

$$
C_{\alpha x_{i} h}^{*}=J_{\alpha x_{i}}+J_{x_{i} h}^{*}
$$

onde $C_{\alpha x_{i} h}^{*}$ é o custo mínimo para se obter o destino $h$ partindo do cruzamento $\alpha$ passando por $x_{i}$, $J_{\alpha x_{i}}$ é o custo de se mover de $\alpha$ até $x_{i}$ e $J_{x_{i} h}^{*}$ é o custo mínimo para alcançar $h$ estando em $x_{i}$.

Além disso, a decisão ótima, ou controle ótimo, a ser tomada em $\alpha, u^{*}(\alpha)$, é a decisão que faz

$$
J_{\alpha h}^{*}=\min _{u^{*}(\alpha)}\left\{C_{\alpha x_{i} h}^{*}, i \in I\right\} .
$$

As equações obtidas através do exame do exemplo acima sintetizam a aplicação do princípio da programação dinâmica no caso do problema em questão.

Uma vez que o princípio da programação dinâmica esteja devidamente introduzido, estamos prontos para dar início à aplicação mais importante no contexto deste trabalho - sua relação com a solução da equação de Hamilton-Jacobi.

\subsection{O princípio da programação dinâmica e as equações de HJ}

Consideremos um processo descrito pela equação diferencial ordinária

$$
\dot{x}=f(x(t), u(t), t) \text { em } \Omega \subset \mathbb{R}^{n},
$$


onde $x(t) \in \mathbb{R}^{n}, u(t) \in \mathbb{R}^{m}$ e $t \geq 0$ com condições iniciais fixas

$$
x\left(t_{0}=0\right)=x_{0} .
$$

Suporemos que $f$ é uma função Lipschtziana com relação à primeira coordenada.

Associemos a esta dinâmica uma função de pagamento dada por

$$
\mathcal{P}\left(x_{0}, u(t)\right)=\int_{t_{0}}^{T} g(x(s), u(s), t) d s+h(x(T), T) .
$$

Nosso exercício principal será demonstrar que a aplicação dada por

$$
v(T, x(T))=\inf _{u \in \mathcal{U}} \mathcal{P}\left(x_{0}, u(t)\right)
$$

é uma solução da equação de Hamilton-Jacobi associada ao problema de otimização acima, a saber,

$$
v_{t}-\max _{v \in \mathcal{V}}[D v \cdot f-g]=0,
$$

chamada equação de Hamilton-Jacobi-Bellman.

Antes, entretanto, de enunciarmos e demonstrarmos o principal resultado que relaciona o princípio da programação dinâmica com a solução do problema de otimização acima, algumas proposições preliminares se fazem necessárias. Passemos a elas.

Proposição 3 (O Teorema de Taylor) Seja $D \in \mathbb{R}^{n}$ um aberto e $f \in \mathcal{C}^{n}(D)$ uma aplicação a valores reais. Suponha que $f$ possui derivadas parciais de ordem $n$ contínuas em uma vizinhança de cada ponto da reta que liga dois quaisquer elementos $u, v \in D$. Então, existe um ponto $\bar{u}$ sobre tal reta tal que vale

$$
f(v)=f(u)+\sum_{i=1}^{n} \frac{1}{i !} D^{i} f(\bar{u})(v-u)^{i} .
$$

Demonstração. Seja $I=[0,1]$, como é usual, e considere a aplicação $F: I \longrightarrow \mathbb{R}$ dada por

$$
F(t)=f(u+t(v-u))
$$

Da hipótese de existência das $n$-ésimas derivadas de $f$, obtemos que $F$ também possui derivadas de ordem $n$ contínuas, as quais são obtidas pela simples aplicação da regra da cadeia e são dadas por

$$
F^{(n)}=D^{n} f(u+t(v-u))(v-u)
$$


Aplicando a versão unidimensional mais simples do teorema de Taylor à função $F$ no intervalo $I$, obtemos $\tau \in I$ tal que

$$
F(1)=F(0)+\sum_{i=1}^{n} \frac{1}{i !} F^{(i)}(\tau) .
$$

Uma vez que $F(1)=f(v)$ e $F(0)=f(u)$, basta então tomarmos $\bar{u}=u+\tau(v-u)$ o que implica que

$$
f(v)=f(u)+\sum_{i=1}^{n} \frac{1}{i !} D^{(i)} f(u+\tau(v-u))(v-u)
$$

i.e.,

$$
f(v)=f(u)+\sum_{i=1}^{n} \frac{1}{i !} D^{(i)} f(\bar{u})(v-u),
$$

e, assim, o resultado está demonstrado.

Esta proposição, apesar de bastante familiar ao estudo elementar da Análise em $\mathbb{R}^{n}$ foi repetida aqui pela sua importância na demonstração do princípio da programação dinâmica e, para facilitar o entendimento do leitor acerca da necessidade de alternativas a este princípio no contexto em que $f$, por exemplo, não é continuamente diferenciável - é justamente neste caso que a noção de solução viscosa aparece.

Vejamos agora uma demonstração do princípio da programação dinâmica.

Proposição 4 (O Princípio da Programação Dinâmica) Considere o problema de otimização da função de pagamento dada em 4.2 para o qual seja $\bar{x}$ um estado ótimo associado ao controle ótimo $\bar{u}$. Então, as restrições $\bar{x}_{\tau}$ e $\bar{u}_{\tau}$, ou seja, a restrição do estado e do controle ótimo ao subintervalo ]$\tau, T[$ são respectivamente uma trajetória ótima e um controle ótimo do problema $v(\tau, \bar{x}(\tau))$.

Demonstração. Suponha, por absurdo, que o par $\left(\bar{x}_{\tau}, \bar{u}_{\tau}\right)$ não seja ótimo em vista do problema $v(\tau, x(T))$. Então, deve existir um par $(\hat{x}, \hat{u})$ tal que

$$
\int_{\tau}^{T} g(\hat{x}(s), \hat{u}(s), s) d s+h(\hat{x}(T), T)<\int_{\tau}^{T} g(\bar{x}(s), \bar{u}(s), s) d s+h(\bar{x}(T), T) .
$$

Considere então o par de controles ótimos dado por $\left(x^{*}, u^{*}\right)$ onde $x^{*}=\bar{x}(s)$ se $\left.s \in\right] t, \tau[$ e $x^{*}=\hat{x}(s)$ se $s \in(\tau, T)$ e $u^{*}=\bar{u}(s)$ se $s \in(t, \tau)$ e $u^{*}=\hat{u}(s)$ se $s \in(\tau, T)$. Então temos que

$$
\begin{array}{r}
v(t, x(T)) \leq \int_{t}^{\tau} g(\bar{x}(s), \bar{u}(s), s) d s+\int_{\tau}^{T} g(\hat{x}(s), \hat{u}(s), s)+h(\hat{x}(T), T) \\
<\int_{t}^{\tau} g(\bar{x}(s), \bar{u}(s), s) d s+\int_{\tau}^{T} g(\bar{x}(s), \bar{u}(s), s)+h(\bar{x}(T), T)=v(t, x(T)),
\end{array}
$$

o que é um absurdo. Assim, como $\tau$ é arbitrário, segue o resultado.

Uma vez que tenhamos à nossa disposição estes elementos, estamos prontos para o enunciado e a demonstração do teorema segundo o qual a função de valor do problema de otimização discutido 
nesta subseção é a solução clássica da equação diferencial parcial de primeira ordem HamiltonJacobi.

Teorema 10 Considere o problema de otimização dado por 4.1 e 4.2 e suponha que

$$
v:[t, T] \times \mathbb{R}^{n} \longrightarrow \mathbb{R}
$$

seja continuamente diferenciável. Então v é uma solução clássica da equação de Hamilton-Jacobi dada por

$$
v_{t}-\max _{v \in \mathcal{V}}(D v \cdot f-g)
$$

com a condição terminal

$$
v(T, x(T))=h(x(T), T)
$$

Demonstração. O primeiro passo, e mais elementar, é demonstrarmos a condição terminal. De fato,

$$
v(T, x(T))=\inf _{u \in \mathcal{U}} \int_{T}^{T} g(x(s), u(s), s) d s+h(x(T), T)=h(x(T), T) .
$$

Passemos agora à parte da demonstração que $v(t, x(T))$ é solução da equação HJ. Considere um instante de tempo $t^{*}<T$ e tome $\Delta t>0$. Assim, escreveremos o controle $u(t)$ como uma soma de duas novas funções, $u_{1}(t)$ e $u_{2}(t)$,

$$
u(t)=u_{1}(t)+u_{2}(t)
$$

com $u_{1}(t)=u(t)$ para $t \in\left(t^{*}, t^{*}+\Delta t\right)$ e $u_{1} \equiv 0$ em $\left(t^{*}+\Delta t, T\right)$ e $u_{2}(t) \equiv 0$ em $\left(t^{*}, t^{*}+\Delta t\right)$ e $u_{2}(t)=u(t)$ para $t \in\left(t^{*}+\Delta t, T\right)$.

Desta forma, a solução $x(t)$ da equação 4.1 também pode ser escrita em dois passos. É fácil ver que $x(t)=x_{u_{1}}(t)$ é uma solução de

$$
\dot{x}(t)=f\left(x(t), u_{1}(t), t\right) \text { em }\left(t^{*}, t^{*}+\Delta t\right)
$$

com a condição

$$
x\left(t^{*}\right)=\xi
$$

Por outro lado, ainda é possível verificar que $x(t)=x_{u_{1}}\left(t, u_{2}\right)$ é uma solução de

$$
\dot{x}(t)=f\left(x(t), u_{2}(t), t\right) \text { em }\left[t^{*}+\Delta t, T\right)
$$

com a condição inicial

$$
x\left(t^{*}+\Delta t\right)=x_{u_{1}}\left(t^{*}+\Delta t\right) .
$$


Tomamos agora

$$
v\left(t^{*}+\Delta t, x_{u_{1}}\left(t^{*}+\Delta t\right)\right)=\inf _{u_{2} \in \mathcal{U}}\left\{\int_{t^{*}+\Delta t}^{T} g\left(x_{u_{1}}\left(s, u_{2}(s)\right), u_{2}(s), s\right) d s+h\left(x\left(T, u_{2}(T)\right), T\right)\right\},
$$

o que nos permite escrever a função $v$ como sendo

$$
v\left(t^{*}, \xi\right)=\inf _{u_{1} \in \mathcal{U}}\left\{\int_{t^{*}}^{t^{*}+\Delta t} g\left(x_{u_{1}}(s), u_{1}(s), s\right) d s+v\left(t^{*}+\Delta t, x_{u_{1}}\left(t^{*}+\Delta t\right)\right)\right\},
$$

uma vez que

$$
\begin{array}{rl}
\int_{t^{*}}^{T} & g(x(s), u(s), s) d s+h(x(T), T)= \\
\quad=\int_{t^{*}}^{t^{*}+\Delta t} g\left(x_{u_{1}}(s), u_{1}(s), s\right) d s+\int_{t^{*}+\Delta t}^{T} g\left(x_{u_{2}}\left(s, u_{2}\right), u_{2}(s), s\right) d s+h\left(x_{u_{2}}\left(T, u_{2}\right), T\right),
\end{array}
$$

sendo que o ínfimo é tomado primeiro com relação a $u_{2}$ com $u_{1}$ fixo e, em seguida, com relação a $u_{1}$.

Aplicando o teorema de Taylor a $x_{u_{1}}\left(t^{*}+\Delta t\right)$, obtemos

$$
x_{u_{1}}\left(t^{*}+\Delta t\right)=x_{u_{1}}\left(t^{*}\right)+\Delta t \dot{x}_{u_{1}}\left(t^{*}\right)+o(\Delta t)=\xi+\Delta t\left(\dot{x}_{u_{1}}\left(t^{*}\right)+\frac{o(\Delta t)}{\Delta t}\right) .
$$

\section{Coloquemos}

$$
\Delta \xi=\Delta t\left(\dot{x}_{u_{1}}\left(t^{*}\right)+\frac{o(\Delta t)}{\Delta t}\right)
$$

Por outro lado, como $v$ é continuamente diferenciável, por hipótese, podemos aplicar o mesmo teorema de Taylor a $v\left(t^{*}+\Delta t, \xi+\Delta \xi\right)$ obtendo

$$
\begin{aligned}
v\left(t^{*}+\Delta t, \xi+\Delta \xi\right)= & v\left(t^{*}, \xi\right)+D v\left(t^{*}, \xi\right) \cdot \Delta \xi+v_{t}\left(t^{*}, \xi\right) \cdot \Delta t+o(\Delta t, \Delta \xi)= \\
& =v\left(t^{*}, \xi\right)+\left[D v\left(t^{*}, \xi\right) \cdot \dot{x}_{u_{1}}\left(t^{*}\right)+v_{t}\left(t^{*}, \xi\right)\right] \Delta t+o(\Delta t)
\end{aligned}
$$

Observe que $\dot{x}_{u_{1}}\left(t^{*}\right)=f\left(x_{u_{1}}\left(t^{*}\right), u_{1}\left(t^{*}, t^{*}\right)=f\left(\xi, u_{1}\left(t^{*}\right), t^{*}\right)\right.$, o que implica em

$$
\begin{array}{r}
\int_{t^{*}}^{t^{*}+\Delta t} g\left(x_{u_{1}}(s), u_{1}(s), s\right) d s=g\left(x_{u_{1}}\left(t^{*}\right), u_{1}\left(t^{*}\right), t^{*}\right) \Delta t+o(\Delta t)= \\
=g\left(\xi, u_{1}\left(t^{*}\right), t^{*}\right) \Delta t+o(\Delta t) .
\end{array}
$$

Reunindo as duas últimas expressões acima obtemos:

$$
\int_{t^{*}}^{t^{*}+\Delta t} g\left(x_{u_{1}}(s), u_{1}(s), s\right) d s+v\left(t^{*}+\Delta t, \xi+\Delta \xi\right)=
$$




$$
=v\left(t^{*}, \xi\right)+\Delta t\left[g\left(\xi, u_{1}\left(t^{*}\right), t^{*}\right)+D v\left(t^{*}, \xi\right) \cdot f\left(\xi, u_{1}\left(t^{*}, t^{*}\right)+v_{t}\left(t^{*}, \xi\right)\right]+o(\Delta t),\right.
$$

o que implica em

$$
v_{t}\left(t^{*}, \xi\right)=\max _{u_{1} \in \mathcal{U}}\left[-D v\left(t^{*}, \xi\right) \cdot f\left(\xi, u_{1}\left(t^{*}\right), t^{*}\right)+g\left(\xi, u_{1}\left(t^{*}\right), t^{*}\right)\right]+\frac{o(\Delta t)}{\Delta t} .
$$

Tomando o limite em 4.5 quando $\Delta t \longrightarrow 0$ segue o resultado.

O resultado acima tem diversas implicações importantes para problemas de maximização envolvendo sistemas controláveis. A primeira delas é a garantia de que no caso de uma função de valor continuamente diferenciável, esta poderá ser obtida analiticamente. A segunda, e mais importante, é que, ao obtermos tal expressão, podemos implementar um mecanismo retroativo, através de uma aplicação retroativa, para obtermos a função de controle $\mathbf{u}(\cdot)$ que resolve o problema de otimização em questão. Isto é feito através de um procedimento conhecido como closed loop control ou feed back map, ao qual iremos nos referir por aplicação retroativa.

Assumindo que, para cada par $(t, \xi)$ exista um único controle que resolve o problema de otimização relativo ao jogo sob estudo, chamamos este tal controle de $\mathbf{u}=D^{\#}(t, \xi)$. A aplicação $D^{\#}$ associa a cada ponto da trajetória ótima $\overline{\mathbf{x}}(t)$ o controle ótimo $\overline{\mathbf{u}}(t)$ que garante a otimalidade do sistema até o instante terminal.

Teorema 11 Considere o problema de otimização sugerido pelas equações 4.1- 4.2 e suponha que exista um único controle $D(t, \xi)$ que o resolve. Considere ainda que a função de valor associada $v$ seja continuamente diferenciável com relação ao tempo. Então, para cada instante do tempo, o estado ótimo $\bar{x}(t)$ e o controle ótimo $\bar{u}(t)$ estão relacionados por

$$
\bar{u}(t)=D(t, \bar{x}(t))
$$

Demonstração. Considere a equação diferencial

$$
\dot{z}(s)=f(z(s), D(s, z(s)), s)
$$

sujeita à condição inicial

$$
z\left(t_{0}\right)=\xi
$$

Considere ainda $v(s, z(s))$. Diferenciando com relação a $s$, obtemos:

$$
\begin{aligned}
& \frac{d v}{d s}(s, z(s))=v_{s}(s, z(s))+D v(s, z(s)) \cdot \dot{z}(s)= \\
& \quad=v_{s}(s, z(s))+D v(s, z(s)) \cdot f(z(s), D(s, z(s)), s)
\end{aligned}
$$

Aplicando a definição de $D$ e o teorema anterior, obtemos que 


$$
\begin{aligned}
D v & (s, z(s)) \cdot f(z(s), D(s, z(s)), s)= \\
& =-g(z(s), D(s, z(s)), s)-\max _{u \in \mathcal{U}}[D v(s, z(s)) \cdot f(z(s), u(s), s)-g(z(s), u(s), s)]= \\
& =-g(z(s), D(, z(s)), s)-v_{s}(s, z(s)),
\end{aligned}
$$

o que implica, diretamente, que

$$
v_{s}(s, z(s))=-g(z(s), D(s, z(s)), s)
$$

Agora, integramos sobre o aberto $] t_{0}, T[$ e obtemos

$$
\begin{array}{r}
v(T, z(T))-v\left(t_{0}, z\left(t_{0}\right)\right)=h(z(T), T)-v\left(t_{0}, \xi\right)= \\
=-\int_{t_{0}}^{T} g(z(s), D(s, z(s)), s) d s .
\end{array}
$$

Ao re-arranjarmos os termos, obtemos sem qualquer dificuldade

$$
v\left(t_{0}, \xi\right)=\int_{t_{0}}^{T} g(z(s), D(s, z(s)), s) d s+h(z(T), T) .
$$

Isto implica que a solução $z(t)$, que resolve a equação diferencial para o controle $u(t)=$ $D(t, z(t))$, é a trajetória ótima. Assim, o par $(z(t), u(t))$ fornece, respectivamente, o estado e o controle ótimo do problema em questão.

Uma hipótese fortemente utilizada em todas as demonstrações deste capítulo diz respeito à regularidade da função $v$. Em outras palavras, sempre supomos que $v$ é uma função de classe $\mathcal{C}^{1}$ sobre o devido domínio. Entretanto, em exemplos até triviais, esta hipótese pode não ser verificada.

Neste caso, o arcabouço acima exposto não poderá ser utilizado pois a função de valor do problema de otimização não será mais uma solução clássica da equação de Hamilton-Jacobi. Vejamos um exemplo simples para motivar a necessidade de alternativas ao conceito de solução clássica da equação de HJ no contexto de problemas de otimização.

Exemplo 8 Considere $\Omega \subset \mathbb{R}, \mathcal{U}=\{-1,1\}$ e suponha que $f: \Omega \times \mathcal{U} \longrightarrow \mathcal{U}$ é dada por

$$
f(x(t), a(t))=a(t)
$$

Tome então o seguinte sistema

$$
\dot{x}(t)=f(x(t), a(t))=a(t)
$$

com a condição inicial $x(0)=\xi$. 
A este sistema associe o funcional de pagamento dado por

$$
J(\xi, a(t))=\int_{0}^{+\infty} l(x(s), a(s)) d s=\int_{0}^{+\infty} l(x(s)) d s
$$

tal que

$$
\begin{gathered}
l(x)=l(-x), \\
\max _{x} l(x)=l(0)>0 .
\end{gathered}
$$

Mais ainda, fixe $R>0$. Então suponha que

$$
l \equiv 0 \operatorname{para}|x|>R
$$

$e$

$$
x l^{\prime}(x)<0 \text { para }|x|<R .
$$

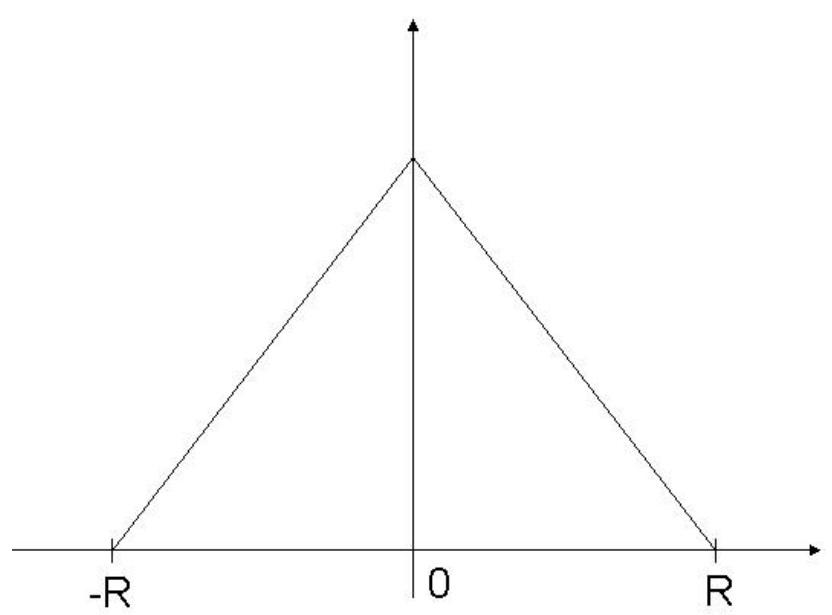

Figura 4.2: Gráfico de uma função com as propriedades de $l$ para dado $R>0$.

Uma vez que nosso objetivo é minimizar $J$, observemos o que ocorre quando $\xi$ é positivo ou negativo. Se for $\xi>0$ e sua derivada for negativa, então ele está caindo, e deverá chegar ao ponto 0 onde $l$ atinge seu máximo. Por outro lado, se for $\xi<0$ e sua derivada for positiva, então ele subirá, alcançará o zero e novamente estaremos em um ponto de máximo de l. Assim, vemos que nosso controle ótimo $\alpha(t)$, em cada instante do tempo, deve ser

$$
\alpha(t) \equiv \operatorname{sgn}(\xi)
$$

Desta forma, obtemos $v(\xi)$ que dependerá do sinal de $\xi$. Vejamos:

$$
v(\xi)=\int_{0}^{+\infty} l(\xi-s) d s \text { se } \xi<0
$$




$$
\begin{array}{r}
v(\xi)=\int_{0}^{+\infty} l(\xi+s) d s \text { se } \xi>0 \\
v(\xi)=\int_{0}^{+\infty} l(-s) d s=\int_{t_{0}}^{+\infty} l(s) d s \text { se } \xi=0 .
\end{array}
$$

Calculando as derivadas laterais de $v$ em zero, temos que

$$
\begin{gathered}
v_{+}^{\prime}(0)=\lim _{x \longrightarrow 0^{+}} \frac{v(x)-v(0)}{x}=\int_{0}^{+\infty} l^{\prime}(t) d t \\
v_{-}^{\prime}(0)=\lim _{x \longrightarrow 0^{-}} \frac{v(x)-v(0)}{x}=\int_{0}^{+\infty} l^{\prime}(-t) d t .
\end{gathered}
$$

Assim, uma vez que $l(\xi)=l(-\xi)$, temos $l^{\prime}(-x)=-l^{\prime}(x)$, e, com isso, $v$ não é diferenciável em $\xi=0$.

De acordo com Bardi e Capuzzo-Dolcetta [10] a equação de HJ para este problema é dada por

$$
\left|v^{\prime}(\xi)\right|-l(\xi)=0
$$

o que não faz sentido clássico em $\xi=0$.

No capítulo seguinte expomos a teoria das soluções viscosas da equação de Hamilton-Jacobi e no capítulo 6 será explicitado como, tanto a programação dinâmica, como as soluções viscosas, podem ser utilizadas na abordagem da teoria dos jogos diferenciais.

Cabe mencionar que neste capítulo foi utilizado um problema de maximização envolvendo apenas um controle, ao passo que na teoria dos jogos diferenciais são considerados problemas que envolvem dois parâmetros - as estratégias de cada jogador - com objetivos (maximização e minimização) distintos. Esta escolha é feita aqui para simplificar a exposição. No capítulo 6 aplicaremos estes resultados ao caso dos jogos diferenciais de maneira natural, com pequeno esforço adicional.

A equação de Hamilton-Jacobi abordada neste capítulo, por sua estreita relação com o princípio da programação dinâmica, é chamada de equação de Hamilton-Jacobi-Bellman. No caso dos jogos diferenciais - envolvendo dois parâmetros - o nome dado à equação associada é Hamilton-JacobiIsaacs, após Rufus Isaacs, que lançou as bases da formulação dos jogos diferenciais entre as décadas de 1950 e 1960. 


\section{Capítulo 5}

\section{Soluções Viscosas da Equação de Hamilton-Jacobi}

\subsection{Introdução às equações de Hamilton-Jacobi}

Neste capítulo discutiremos a existência e unicidade de soluções viscosas da equação de HamiltonJacobi. No entanto, nesta seção inicial, vamos expor alguns fatos acerca destas equações.

Consideremos o seguinte problema de valores iniciais:

$$
\left\{\begin{array}{cc}
u_{t}+H(x, D u) & \text { em } \mathbb{R}^{n} \times(0, \infty) \\
u \equiv \phi & e m \mathbb{R}^{n} \times\{0\}
\end{array}\right.
$$

onde $u: \mathbb{R}^{n} \times[0, \infty) \longrightarrow \mathbb{R}$ é a incógnita, e $D u$ denota as $n$ derivadas de $u$ com respeito às componentes de $x$ ao passo em que $u_{t}$ denota a derivada de $u$ com relação a $t$, como usual. Nosso interesse é discutir em algum detalhe a função $H$ no problema de Cauchy acima.

Ao escrevermos o sistema de equações ordinárias características ${ }^{1}$ para este problema temos:

$$
\left\{\begin{array}{c}
\dot{x}=D_{p} H(x, p) \\
\dot{p}=-D_{x} H(x, p) .
\end{array}\right.
$$

Vamos oferecer agora uma interpretação da aplicação $H$.

Seja $L: \mathbb{R}^{n} \times \mathbb{R}^{n} \longrightarrow \mathbb{R}$ uma função tão regular quanto necessário, por exemplo, de classe $\mathcal{C}^{\infty}\left(\mathbb{R}^{2 n}\right)$. Fixemos agora dois pontos em $\mathbb{R}^{n}, x$ e $y$ e um instante terminal $T$ e definamos o funcional $J: \mathcal{A} \longrightarrow \mathbb{R}$ por

$$
J(w) \doteq \int_{0}^{T} L(\dot{w}(s), w(s)) d s,
$$

onde $\mathcal{A}=\left\{w \in \mathcal{C}^{2}([0, T]) \mid w(0)=y, w(T)=x\right\}$.

Definição 38 L, como definida acima, será chamada Lagrangiano.

A idéia é que um indivíduo quer se deslocar entre os pontos $x$ e $y$ - ou uma partícula deve ser deslocada, ou qualquer coisa que se queira deslocar - durante o intervalo de tempo $0-T$ e isto deve ser feito de modo a otimizar um funcional de performance, na nossa notação, $J$. Os elementos

\footnotetext{
${ }^{1}$ Para uma exposição das noções de curvas características e suas aplicações na resolução de equações parciais de primeira ordem, sugerimos os textos de Fritz John [34] e Lawrence Evans [22].
} 
do conjunto $\mathcal{A}$ são as possíveis trajetórias conectando os pontos de partida e destino. A figura 5.1 ilustra a idéia do deslocamento e exemplifica trajetórias possíveis.

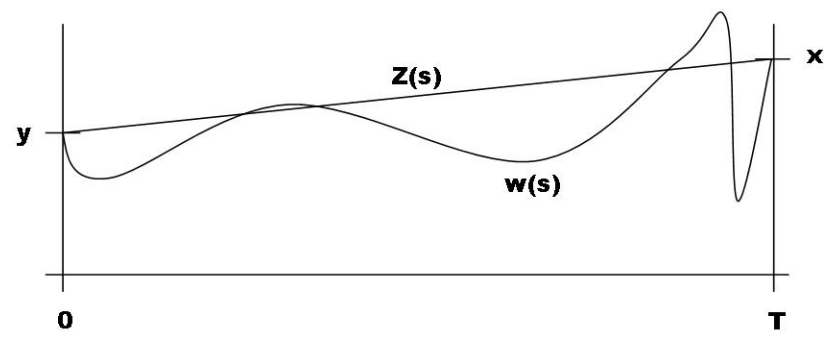

Figura 5.1: Problema de deslocamento e exemplo de trajetórias.

O interesse aqui é encontrar o ponto $x \in \mathcal{A}$ tal que

$$
J(x)=\inf _{w \in \mathcal{A}} J(w)
$$

Veremos que - quando existir - este ponto será a solução da primeira equação do sistema de equações características dado acima.

Lema 4 A trajetória ótima x para 5.1 é uma solução da equação

$$
-\frac{d}{d s}\left(D_{p} L(\dot{x}(s), x(s))\right)+D_{x} L(\dot{x}(s), x(s))=0
$$

para $s \in(0, T)$. Esta é a chamada equação de Euler-Lagrange associada a L.

Demonstração. Considere uma trajetória ótima $x$ e tome uma função $v:[0, T] \longrightarrow \mathbb{R}^{n}$ que seja de classe $\mathcal{C}^{\infty}$ sobre $[0, T]$ e tal que

$$
v(0)=v(T)=0
$$

Defina então $w: \mathbb{R} \times[0, T] \longrightarrow \mathbb{R}^{n}$ como sendo a aplicação

$$
w(\tau, s)=x(s)+\tau v(s)
$$

É fácil vermos que $w(\cdot, 0)=y$ e $w(\cdot, T)=x$. Por preservar a regularidade de $x$, vemos que $w \in \mathcal{A}$. Assim, $w$ é, per se, uma trajetória admissível. Observe no entanto que

$$
J(x) \leq J(w)
$$

Ao definirmos a função

$$
i(\tau) \doteq J(x+\tau v)
$$

observamos que esta tem um ponto de mínimo em $\tau=0$ e, portanto,

$$
\frac{d}{d \tau} i(0)=0
$$


Calculando explicitamente esta derivada, encontramos que

$$
0=\sum_{j=i}^{n} \int_{0}^{T}\left[-\frac{d}{d s}\left(L_{q_{j}}(\dot{x}, x)\right)+L_{x_{i}}(\dot{x}, x)\right] v_{j} .
$$

Como esta igualdade é válida para qualquer função $v \in \mathcal{C}^{\infty}([0, T])$, segue que, para todo $j=$ $1, \ldots, n$,

$$
-\frac{d}{d s}\left(L_{q_{j}}(\dot{x}, x)\right)+L_{x_{i}}(\dot{x}, x)=0,
$$

de onde segue a afirmação do lema.

Estamos muito próximos da discussão de um resultado que, essencialmente, permeia todo o trabalho. Temos visto que, grosso modo, no contexto dos jogos diferenciais, a solução da equação de HJ é a função de valor do jogo. Ou seja, a solução da HJ associada ao jogo reflete um caráter de otimalidade dos controles, no sentido em que estes minimizam ou maximizam um funcional de desempenho.

Nosso intuito com a discussão desta seção é expor o leitor ao fato de que a conexão das equações de HJ com problemas de otimização tem sua origem no estudo da Mecânica e representa uma elegante aplicação das técnicas da teoria das equações diferenciais a problemas de cálculos de variações e controle ótimo.

Vejamos duas definições:

Definição 39 Seja $L: \mathbb{R}^{n} \longrightarrow \mathbb{R}$. A transformada de Legendre de L, que será denotada por $L^{*} e ́$

$$
L^{*} \doteq \sup _{q \in \mathbb{R}^{n}}\{p \cdot q-L(q)\}
$$

Definição 40 Seja $H=L^{*}$. H será chamado Hamiltoniano.

Nosso próximo resultado afirma que, sob condições bastante gerais, a trajetória ótima do problema de otimização de $J$ é a solução do sistema de EDO's características associado à equação de HJ cujo Hamiltoniano ${ }^{2}$ é dado como na definição acima.

Proposição 5 Seja $p \doteq D_{q} L(x, q)$ e assuma que esta equação possa ser resolvida unicamente em termos de $q=q(x, p)$. Considere ainda $H$ como na definição anterior. Então o par $(\dot{x}, \dot{p})$ formado pela trajetória ótima e pela função p resolvem o sistema:

$$
\left\{\begin{array}{c}
\dot{x}=D_{p} H(x, p) \\
\dot{p}=-D_{x} H(x, p) .
\end{array}\right.
$$

\footnotetext{
${ }^{2}$ Willian Rowan Hamilton nasceu um Dublin a 4 de agosto de 1805. Trabalhou no Trinity College dedicando-se sobretudo à Mecânica Clássica, adaptando alguns de seus métodos a problemas da Ótica. Além de dividir o título de um importante teorema em Álgebra Linear com Cayley, é ainda recordado pela criação dos quatérnios e pela cunhagem do termo 'tensor'. Morreu também em Dublin, a 2 de setembro de 1865 . Por falar em cunhagem, o bi-centenário de seu falecimento foi celebrado na Irlanda com a cunhagem de uma moeda que leva o símbolo Nabla cujo uso também é devido a Hamilton.
} 
O sistema acima é chamado sistema Hamiltoniano.

Demonstração. É claro das definições que

$$
\dot{x}(s)=q(x(s), p(s)) .
$$

Observe que

$$
\frac{\partial H}{\partial x_{i}}(x, p)=\sum_{j=1}^{n} p_{j} \frac{\partial q_{j}}{\partial x_{i}}(x, p)-\frac{\partial L}{\partial q_{j}}(x, q) \frac{\partial q_{j}}{\partial x_{i}}(x, p)-\frac{\partial L}{\partial x_{i}}(x, q),
$$

o que implica que, pela definição de $p$,

$$
\frac{\partial H}{\partial x_{i}}(x, p)=-\frac{\partial L}{\partial x_{i}}(x, q) .
$$

Por outro lado,

$$
\frac{\partial H}{\partial p_{i}}(x, p)=q_{i}(x, p)+\sum_{j=1}^{n} p_{j} \frac{\partial q_{j}}{\partial x_{i}}(x, p)-\frac{\partial L}{\partial q_{j}}(x, q) \frac{\partial q_{j}}{\partial x_{i}}(x, p),
$$

o que, pela mesma razão, implica

$$
\frac{\partial H}{\partial p_{i}}=q_{i}(x, p)
$$

Mas, da última igualdade, segue

$$
\frac{\partial H}{\partial p_{i}}=\dot{x}_{i}(x, p)
$$

o que verifica a validade da proposição para as $n$ primeiras equações do sistema.

Entretanto, como

$$
-\frac{\partial L}{\partial x_{i}}(\dot{x}(s), x(s))=-\frac{\partial}{\partial s}\left(\frac{\partial L}{\partial q_{i}}(\dot{x}(s), x(s))\right)=-\dot{p}_{i}(s)
$$

segue a validade da proposição para as demais $n$ equações do sistema Hamiltoniano de EDO's.

Assim, apresentamos a primeira conexão entre as equações de HJ com problemas de otimização e cálculo de variações.

O restante deste capítulo será dedicado à discussão das soluções viscosas da equação de HamiltonJacobi e, através da seguinte exposição esperamos oferecer os elementos necessários ao entendimento da aplicação destas ferramentas à teoria dos jogos diferenciais.

\subsection{Primeiras idéias e alguns exemplos}

A noção de solução viscosa está associada à necessidade de procurarmos por funções que de alguma maneira resolvam um determinado problema de Cauchy envolvendo derivadas parciais, mas que não atendam a todas as hipóteses que em geral são feitas sobre soluções chamadas clássicas, por exemplo, não sendo diferenciáveis em algum, ou em nenhum ponto.

Algumas referências importantes a respeito da noção de solução viscosa, bem como das suas 
propriedades e suas relações com a teoria dos jogos diferenciais podem ser obtidas nos trabalhos de Evans [21], Crandall, Evans e Lions [42], Crandall e Lions [16] e [17], Gomes [27] e [26] e Souganidis [51].

Vejamos um exemplo de classes de soluções alternativas às clássicas. Consideremos o problema de valores iniciais abaixo:

$$
\frac{\partial u}{\partial t}+\frac{1}{2} \frac{\partial\left(u^{2}\right)}{\partial x}=0
$$

em $\mathbb{R} \times(0, \infty)$ sob as condições iniciais $u(x, 0)=g(x)$ para todo $x \in \mathbb{R}$.

Este problema envolve a equação de Burger - de segunda ordem, linear, do tipo parabólico bastante familiar ao estudo da mecânica de fluídos. De acordo com [22], em geral, não é possível encontrarmos solução clássica para este problema, ainda que a condição inicial tenha alto grau de regularidade. Ao invés disto, vamos procurar por um tipo particular de solução fraca.

Seja $\Phi$ uma função teste - i.e., de classe $\mathcal{C}^{1}$ e de suporte compacto - e suponha que $u$ satisfaça 5.2. Multiplicando 5.2 por $\Phi$ e integrando por partes obtemos

$$
\int_{\mathbb{R}} \int_{0}^{\infty}\left(u \frac{\partial \Phi}{\partial t}+\frac{u^{2}}{2} \frac{\partial \Phi}{\partial x}\right)(x, t) d t d x+\int_{\mathbb{R}} u(x, 0) \Phi(x, 0) d x=0 .
$$

Uma vez que $u \in L^{1}\left(\mathbb{R} \times \mathbb{R}^{+}\right)$, a expressão acima faz sentido e então, dizemos que uma função $u$ é uma solução fraca de 5.2 no sentido de distribuições caso a igualdade 5.3 valha para toda função teste $\Phi$. Vale a pena mencionar que, caso possamos encontrar soluções fracas no sentido de distribuições para uma determinada EDP, esta estará na chamada forma divergente. Do contrário, diz-se tratar da forma não-divergente.

Apesar deste exemplo inicial, no caso das equações de Hamilton-Jacobi não podemos utilizar da noção de solução fraca no sentido de distribuições (ver [38]). Sendo assim, vamos procurar soluções fracas de outro aspecto, a saber, as soluções de viscosidade. Vejamos um exemplo.

Consideremos a equação diferencial parcial unidimensional

$$
1-\left(u^{\prime}(x)\right)^{2}=0
$$

$\mathrm{em}]-1,1[$, com

$$
u \equiv 1
$$

em $\{-1,1\}$. É fácil notarmos que

$$
u(x)=|x|
$$

satisfaz à equação a menos da origem, onde $u$ não é diferenciável. Portanto, não pode ser uma solução clássica. Vejamos o gráfico da solução na figura abaixo, cuja mera observação já sugere que esta de fato é uma solução do problema considerado a menos da origem. 


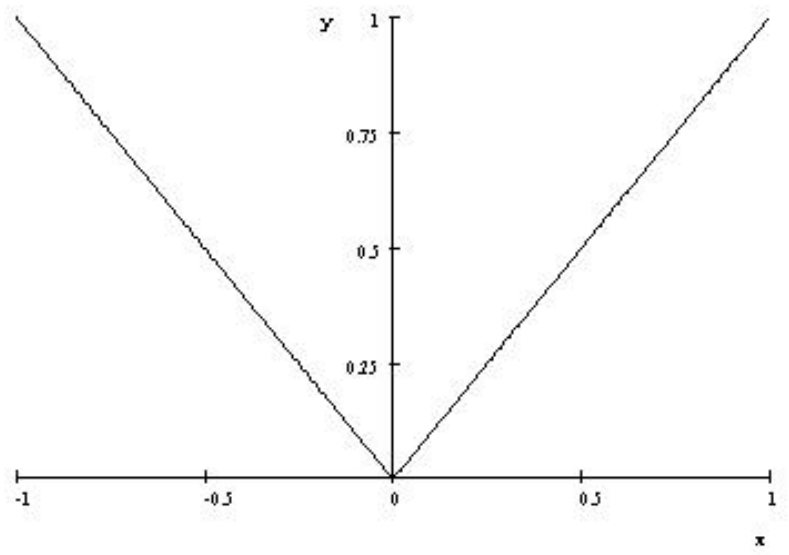

Figura 5.2: Gráfico da função $u(x)=|x|$.

Por outro lado, não podemos utilizar o mecanismo da integração por partes, como sugerido no exemplo anterior. Desta forma, a pergunta natural é se $u(x)=|x|$ é uma solução do problema em algum sentido mais fraco do que o clássico e alternativo ao sentido das distribuições. Veremos mais adiante que, felizmente, esta é uma (de fato a única) solução viscosa do problema em questão.

\subsection{Definições e propriedades}

Apesar do foco deste trabalho centrar-se sobre as equações de Hamilton-Jacobi, em particular, as versões Hamilton-Jacobi-Bellman e Hamilton-Jacobi-Isaacs, proporemos as definições de soluções viscosas, bem como suas principais propriedades de maneira mais geral. Assim, consideramos equações diferenciais parciais de primeira ordem da forma

$$
F(x, u(t, x), D u(t, x))=0 .
$$

Apresentaremos inicialmente as definições de sub-diferencial e super-diferencial, utilizadas na definição de solução viscosa.

Definição 41 Seja $u: \mathbb{R}^{n} \longrightarrow \mathbb{R}$, contínua, e considere os conjuntos

$$
D^{+} u(x)=\left\{p \in \mathbb{R}^{n} \mid \limsup _{y \rightarrow x} \frac{u(y)-u(x)-p \cdot(y-x)}{|y-x|} \leq 0\right\}
$$

$e$

$$
D^{-} u(x)=\left\{p \in \mathbb{R}^{n} \mid \liminf _{y \rightarrow x} \frac{u(y)-u(x)-p \cdot(y-x)}{|y-x|} \geq 0\right\} .
$$

Chamamos $D^{+} u(t, x)$ de conjunto super-diferencial de $u$ no ponto $x$ e $D^{-} u(t, x)$ de conjunto subdiferencial de u no ponto $x$.

De posse desta noção, oferecemos uma primeira definição formal de solução de viscosidade. 
Definição 42 1. Dizemos que uma função contínua u é sub-solução viscosa de

$$
F(x, u(t, x), D u(t, x))=0,
$$

com $x \in \Omega \subset \mathbb{R}^{n}$, se $F(x, u(x, t), p) \leq 0$ para todo $x \in \Omega \subset \mathbb{R}^{n}$ e para todo $p \in D^{+} u(t, x)$.

2. Dizemos que uma função contínua u é super-solução viscosa de

$$
F(x, u(t, x), D u(t, x))=0,
$$

com $x \in \Omega \subset \mathbb{R}^{n}$, se $F(x, u(t, x), q) \geq 0$ para todo $x \in \Omega \subset \mathbb{R}^{n}$ e para todo $q \in D^{-} u(t, x)$.

Se u for super e sub-solução viscosa de $F(t, x, u(t, x), D u(t, x))=0$, então será chamada solução viscosa.

No entanto, uma vez que a grande maioria dos resultados que serão discutidos aqui fazem uso de uma definição alternativa a esta, ofereceremos a segunda definição de viscosidade - que será provada equivalente à primeira.

Definição 43 Considere $\Omega \subset \mathbb{R}^{n}$, aberto, $F \in \mathcal{C}\left(\Omega \times \mathbb{R} \times \mathbb{R}^{n}\right)$ e $u \in \mathcal{C}(\Omega)$. Então dizemos que:

1. u é uma solução de viscosidade de $F(x, u, D u) \leq 0$ em $\Omega$ se, para qualquer função $\phi$ de classe $\mathcal{C}^{1}(\Omega)$ vale que se $x_{0}$ é um ponto de máximo local de $u-\phi$, então

$$
F\left(x_{0}, u\left(x_{0}\right), D \phi\left(x_{0}\right)\right) \leq 0 .
$$

2. u é uma solução de viscosidade de $F(x, u, D u) \geq 0$ em $\Omega$ se, para qualquer função $\phi$ de classe $\mathcal{C}^{1}(\Omega)$ vale que se $x_{0}$ é um ponto de mínimo local de $u-\phi$, então

$$
F\left(x_{0}, u\left(x_{0}\right), D \phi\left(x_{0}\right)\right) \geq 0 .
$$

Novamente, se u satisfizer as condições 1 e 2 acima, chamamos u de solução viscosa do problema $F(t, x, u, D u)=0$ em $\Omega$.

As definições acima não envolvem equações evolucionárias, ou seja, funções $F$ que dependem da variável $t$. Entretanto, estender estas definições para o caso evolucionário é bastante simples. Considere uma equação da forma

$$
u_{t}(x, t)+F\left(t, x, u(x, t), D_{x} u(x, t)\right)=0 .
$$

Então, se forem $y=(x, t) \in D \subset \Omega \times(0, T) \subset \mathbb{R}^{n+1}, H(y, r, q)=q_{n+1}+F\left(t, x, r, q_{1}, \ldots, q_{n}\right) \mathrm{e}$ $q=\left(q_{1}, \ldots, q_{n}, q_{n+1}\right)$ caímos no caso anterior.

Examinemos o exemplo da seção anterior

$$
1-\left(u^{\prime}(x)\right)^{2}=0
$$


$\mathrm{em}]-1,1[, \mathrm{com}$

$$
u \equiv 1
$$

em $\{-1,1\}$ e a função módulo

$$
u(x)=|x| .
$$

Veremos que de fato, esta é uma solução viscosa do problema.

Seja $\phi \in \mathcal{C}^{1}([-1,1])$ e suponha que $x_{0}$ seja um ponto de máximo ou de mínimo de $u-\phi$. Se for $x_{0} \neq 0$, segue que

$$
\left|x_{0}\right|^{\prime}-\phi^{\prime}\left(x_{0}\right)=0
$$

pela condição de otimalidade de $x_{0}$. Assim,

$$
\left(\left|x_{0}\right|^{\prime}\right)^{2}=\left(\phi^{\prime}\left(x_{0}\right)\right)^{2}
$$

o que implica que

$$
1-\left(\phi^{\prime}\left(x_{0}\right)^{2}\right)=0
$$

fornecendo ao mesmo tempo as condições para sub e super-solução viscosa.

Vejamos agora o que ocorre se $x_{0}=0$. Consideremos inicialmente o caso de máximo local, i.e., em que

$$
(u-\phi)(0) \geq(u-\phi)(x)
$$

para todo $x \in]-\varepsilon, \varepsilon[$ para algum $\varepsilon$. Então segue que

$$
-\phi(0) \geq|x|-\phi(x)
$$

ou seja,

$$
|x| \leq \phi(x)-\phi(0)
$$

para todo $x \in]-\varepsilon, \varepsilon[$.

Assim, se for $x<0$ temos

$$
\frac{\phi(x)-\phi(0)}{x} \leq-1
$$

e se for $x>0$ temos

$$
\frac{\phi(x)-\phi(0)}{x} \geq 1
$$

Tomando os limites

$$
\lim _{x \rightarrow 0^{-}} \frac{\phi(x)-\phi(0)}{x}
$$

e

$$
\lim _{x \rightarrow 0^{+}} \frac{\phi(x)-\phi(0)}{x}
$$

observamos que $\phi_{-}^{\prime}(0) \leq-1$ e $\phi_{+}^{\prime}(0) \geq 1$. Por ser $\phi$ diferenciável em todos os pontos, segue o absurdo. Vejamos agora a condição para super-solução viscosa. 
Suponha que $x_{0}=0$ é um ponto de mínimo local. Então

$$
-\phi(0) \leq|x|-\phi(x)
$$

o que implica que

$$
\phi(x)-\phi(0) \leq|x|
$$

Assim, se for $x>0$ temos que

$$
\frac{\phi(x)-\phi(0)}{x} \leq 1
$$

Caso seja $x<0$, temos que

$$
\frac{\phi(x)-\phi(0)}{x} \geq-1
$$

Assim, novamente, tomando limites acima, e, por ser $\phi$ diferenciável, temos que $\left|\phi^{\prime}(0)\right| \leq 1$, o que implica na condição de sub-solução viscosa uma vez que $1-\left(\phi^{\prime}(0)\right)^{2} \geq 0$.

Um fato notório da teoria das equações diferenciais em geral é que se $u$ é uma solução clássica de $F=0$, então $-u$ será uma solução de $-F=0$. Isto não é verdade quando tratamos de soluções viscosas. Tome $u(x)=-|x|$ e considere

$$
\left(u^{\prime}(x)\right)^{2}-1=0
$$

Tomemos $\phi(x)=x^{2}+1$. Então $(u-\phi)(x)=|x|-x^{2}-1$ cujo gráfico é dado na figura abaixo:

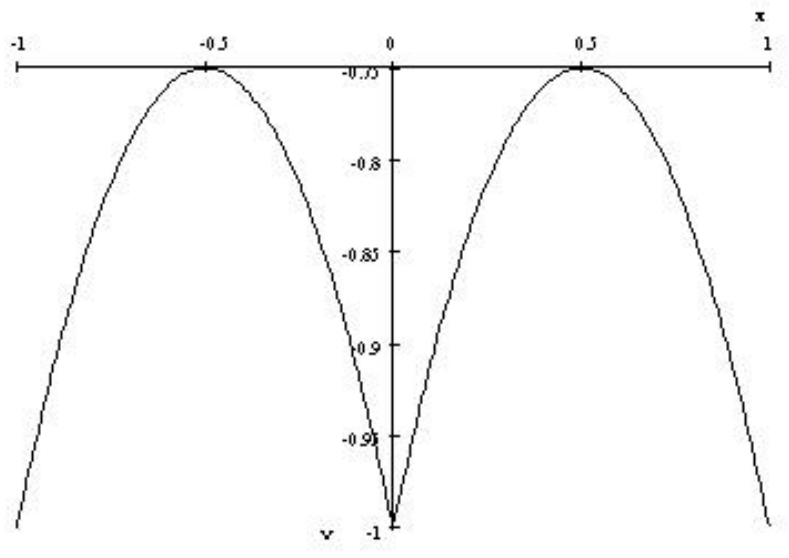

Figura 5.3: Gráfico da função $u(x)=|x|-x^{2}-1$.

Podemos notar que em uma vizinhança de $x=0$, este é um ponto de mínimo. Entretanto, $\phi^{\prime}(0)=0$ implica que

$$
\left(\phi^{\prime}(0)\right)^{2}-1=-1<0,
$$

o que contradiz a condição de sub-solução viscosa.

Este fato não é isolado, sendo uma conseqüência da proposição abaixo.

Proposição 6 Sejam $\Omega \subset \mathbb{R}^{n}$ e $F \in \mathcal{C}\left(\mathbb{R} \times \Omega \times \mathbb{R} \times \mathbb{R}^{n}\right)$. Então são equivalentes: 
1. u é uma solução de viscosidade de $F(t, x, u, D u)=0$.

2. $v=-u$ é uma solução de viscosidade de $-F(t, x, v, D v)=0$.

Demonstração. Para esta demonstração, é suficiente considerarmos a definição de solução viscosa.

Com a finalidade de familiarizar o leitor, bem como demonstrar a equivalência entre as definições 42 e 43 dadas acima, vamos expor algumas propriedades e resultados acerca dos sub e superdiferenciais.

A princípio, nos será útil notar que, dado um ponto $x_{0}, D^{+} u\left(x_{0}\right)=-D^{-}(-u)\left(x_{0}\right)$. Tomemos um ponto $p \in D^{+} u\left(x_{0}\right)$. Pela definição, em alguma vizinhança de $x_{0}$, vale que

$$
\frac{u(x)-u\left(x_{0}\right)-p\left(x-x_{0}\right)}{\left\|x-x_{0}\right\|} \leq 0
$$

Portanto,

$$
\frac{-u(x)+u\left(x_{0}\right)+p\left(x-x_{0}\right)}{\left\|x-x_{0}\right\|} \geq 0
$$

o que implica

$$
\lim \inf _{x \rightarrow x_{0}} \frac{-u(x)+u\left(x_{0}\right)-(-p)\left(x_{x 0}\right)}{\left\|x-x_{0}\right\|} \geq 0 .
$$

Assim, $-p \in D^{-}(-u)\left(x_{0}\right)$, ou seja, $p \in-D^{-}(-u)\left(x_{0}\right)$.

Por outro lado, suponha que $p \in-D^{-}(-u)\left(x_{0}\right)$. Então, em alguma vizinhança do ponto $x_{0}$ podemos assumir válida desigualdade

$$
\frac{-u(x)+u\left(x_{0}\right)+p\left(x-x_{0}\right)}{\left\|x-x_{0}\right\|} \geq 0
$$

o que implica

$$
\frac{u(x)-u\left(x_{0}\right)-p\left(x-x_{0}\right)}{\left\|x-x_{0}\right\|} \leq 0 .
$$

Novamente, pelo mesmo argumento anterior envolvendo limites, concluímos que $p \in D^{+} u\left(x_{0}\right)$. De maneira inteiramente análoga é possível demonstrar que $D^{-} u\left(x_{0}\right)=-D^{+}(-u)\left(x_{0}\right)$.

Passemos agora aos passos que nos permitirão concluir pela equivalência entre as duas definições de soluções viscosas.

Proposição 7 Sejam $\Omega \subset \mathbb{R}^{n}$, aberto, $x_{0} \in \Omega, u \in \mathcal{C}(\Omega)$ e $p \in \mathbb{R}^{n}$. São equivalentes:

1. $p \in D^{+} u\left(x_{0}\right)$ se e somente se existe $\phi \in \mathcal{C}^{1}(\Omega)$ tal que $x_{0}$ é um ponto de máximo local de $u-\phi$ e $D \phi\left(x_{0}\right)=p$.

2. $p \in D^{-} u\left(x_{0}\right)$ se e somente se existe $\phi \in \mathcal{C}^{1}(\Omega)$ tal que $x_{0}$ é um ponto de mínimo local de $u-\phi$ e $D \phi\left(x_{0}\right)=p$. 
Demonstração. Iniciamos a demonstração introduzindo uma função arbitrária $\sigma:[0,+\infty[\longrightarrow \mathbb{R}$ tal que $\sigma$ é contínua, não decrescente, e fixa a origem, i.e., $\sigma(0)=0$. Considere ainda

$$
\rho(r)=\int_{0}^{r} \sigma(t) d t
$$

É fácil ver que $\rho$ é de classe $\mathcal{C}^{1}$. Ainda é fácil vermos que $\rho(0)=\rho^{\prime}(0)=\sigma(0)=0$. Finalmente, considere a figura 5.4 abaixo

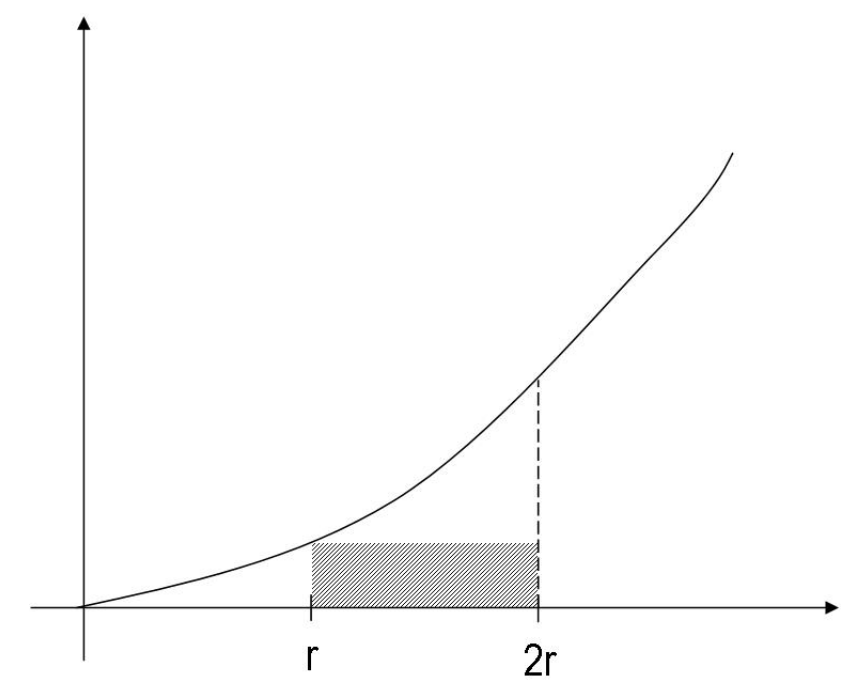

Figura 5.4: Função $\sigma$, comparação de áreas.

Nela, vemos o gráfico de uma função $\sigma$ arbitrária - desde que satisfaça as condições impostas no início da demonstração - e o retângulo hachurado que possui área $\sigma(r) r$. Como este triângulo está inserido na área compreendida entre a curva $\sigma$ e o eixo das abscissas (de 0 até $2 r$ ), temos que

$$
\rho(2 r) \geq \sigma(r) r
$$

Seja $p \in D^{+} u\left(x_{0}\right)$. Então, em alguma vizinhança de $x_{0}$, digamos, $\mathcal{B}_{\varepsilon}\left(x_{0}\right)$ para algum $\varepsilon$ positivo, temos

$$
\frac{u(x)-u\left(x_{0}\right)-p\left(x-x_{0}\right)}{\left\|x-x_{0}\right\|} \leq \sigma\left(\left\|x-x_{0}\right\|\right)
$$

para todo $x \in \mathcal{B}_{\varepsilon}\left(x_{0}\right)$. Rearranjando os termos,

$$
u(x) \leq u\left(x_{0}\right)+p\left(x-x_{0}\right)+\sigma\left(x-x_{0}\right)\left\|x-x_{0}\right\|
$$

Considere agora

$$
\psi(x)=u\left(x_{0}\right)+p\left(x-x_{0}\right)+\rho\left(2\left\|x-x_{0}\right\|\right) .
$$

É fácil ver que $\psi \in \mathcal{C}^{1}$ uma vez que é a soma de funções de classe $\mathcal{C}^{1}$. Mais ainda, $D \psi\left(x_{0}\right)=p$. 
Finalmente, se considerarmos novamente $x \in \mathcal{B}_{\varepsilon}\left(x_{0}\right)$ temos que

$$
u(x)-\psi(x)=u(x)-u\left(x_{0}\right)-p\left(x-x_{0}\right)-\rho\left(2\left\|x-x_{0}\right\|\right)
$$

o que implica que, na vizinhança considerada,

$$
(u-\psi)(x) \leq \sigma\left(\left\|x-x_{0}\right\|\right)\left\|x-x_{0}\right\|-\rho\left(\left\|x-x_{0}\right\|\right) \leq 0=(u-\psi)\left(x_{0}\right) .
$$

Assim, $(u-\psi)$ tem um ponto de máximo local em $x_{0}$.

Para verificarmos a implicação na direção oposta, tomemos a desigualdade

$$
u(x)-\psi(x) \leq u\left(x_{0}\right)-\psi\left(x_{0}\right)
$$

Re-arranjando os termos e subtraindo $D \psi\left(x_{0}\right)\left(x-x_{0}\right)$ de cada lado, temos que

$$
u(x)-u\left(x_{0}\right)-D \psi\left(x_{0}\right)\left(x-x_{0}\right) \leq \psi(x)-\psi\left(x_{0}\right)-D \psi\left(x_{0}\right)\left(x-x_{0}\right) .
$$

Utilizando que $D \psi\left(x_{0}\right)=p$, que o lado direito da expressão acima tende a zero - da definição de diferencial - e que $\left\|x-x_{0}\right\|$ é um número positivo - da definição de norma - segue que

$$
\limsup _{x \rightarrow x_{0}} \frac{u(x)-u\left(x_{0}\right)-p\left(x-x_{0}\right)}{\left\|x-x_{0}\right\|} \leq 0
$$

de onde segue a implicação desejada.

Para o item 2, recordamos ao leitor que $D^{-} u(x)=-\left(D^{+}(-u)(x)\right)$ e o resultado segue imediatamente aplicando o argumento anterior a $-u$.

Corolário 2 As definições 42 e 43, de solução viscosa, acima, são equivalentes.

Demonstração. Mostraremos inicialmente que $u$ é uma sub-solução de viscosidade de $F(x, u, D u)=$ 0 se, e somente se, $F(x, u, p) \leq 0$ para todo $x \in \Omega$ e para todo $p \in D^{+} u(x)$.

Seja $x \in \Omega$ e $p \in D^{+} u(x)$. Da proposição anterior, existe uma função $\psi$ de classe $\mathcal{C}^{1}$ tal que $x$ é máximo local de $(u-\psi)$ e $D \psi(x)=p$. Assim, temos que

$$
F(x, u(x), p)=F(x, u(u), D \psi(x)) \leq 0
$$

onde a desigualdade segue da hipótese de que $u$ é super-solução viscosa do problema considerado.

Deste modo, concluímos que se $u$ é uma solução viscosa de $F=0$, então para todo $x \in \Omega$ e $p \in D^{+} u(x)$, temos que $F(x, u(x), p) \leq 0$, o que nos garante a primeira direção da implicação.

Para a segunda direção da implicação, assuma que para todo $x \in \Omega$ e $p \in D^{+} u(x)$, temos $F(x, u(x), p) \leq 0$ e considere uma função $\psi$ de classe $\mathcal{C}^{1}$ e $x \in \Omega$ tal que $x$ é um ponto de máximo local de $(u-\psi)$. Da proposição anterior, e de $p \in D^{+} u(x)$, segue $D \psi(x) \subset D^{+} u(x)$, o que implica

$$
F(x, u(x), D \psi(x)) \leq 0
$$


de onde segue o resultado.

Assim, temos que $u$ é uma sub-solução de viscosidade de $F(x, u, D u)=0$ se, e somente se, $F(x, u, p) \leq 0$ para todo $x \in \Omega$ e para todo $p \in D^{+} u(x)$.

Resta-nos verificar que se $u$ é uma super-solução viscosa de $F=0$, então para todo $x \in \Omega$ e para todo $p \in D^{-} u(x)$ temos que $F(x, u(x), p) \geq 0$. No entanto, para isto, podemos utilizar o fato de que $D^{-} u(x)=-\left(D^{+}(-u)(x)\right)$ e o resultado seguirá facilmente.

No início desta seção, mencionamos como estender a definição de solução viscosa para o caso da equação evolucionária. Uma questão que fica respondida, então, é que se $u$ é solução viscosa de

$$
u_{t}+F(t, x, u, D u) \leq 0 \quad\left(u_{t}+F(t, x, u, D u) \geq 0\right)
$$

em $\Omega \times(0, T)$, então para qualquer $\phi$ de classe $\mathcal{C}^{1}(\Omega \times(0, T))$ e qualquer ponto de máximo (mínimo) $(x, t) \in \Omega \times(0, T)$ de $u-\phi$ temos que

$$
\phi_{t}(x, t)+F\left(x, t, u(x, t), D_{x} \psi(x, t)\right) \leq 0 \quad\left(\phi_{t}(x, t)+F\left(x, t, u(x, t), D_{x} \psi(x, t)\right) \geq 0\right) .
$$

Naturalmente, caso todas as aplicações envolvidas acima estivessem definidas para $(\cdot, T)$, uma questão seria o comportamento das desigualdades acima em pontos de máximo da forma $\left(x_{0}, T\right)$, com $x_{0} \in \Omega$. A resposta a esta pergunta - de nosso interesse em demonstrações futuras - será dada com base na discussão da noção de estabilidade de um ponto minimizante de uma função.

Considere uma função

$$
f: \mathbb{R}^{n} \longrightarrow \mathbb{R}
$$

e

$$
g(x, \lambda)=f(x)+\lambda h(x)
$$

com $h: \mathbb{R}^{n} \longrightarrow \mathbb{R}^{m}$ e $\lambda \in \mathbb{R}^{m}$. Este último pode ser interpretado como um parâmetro de perturbação, ou seja, quando $\lambda=0$ então $g \equiv f$. Supondo que $f$ e $h$ possuem a regularidade que desejarmos e, mais ainda, que $f$ possui um ponto de mínimo $x_{0}$ tal que a matriz $D^{2} f\left(x_{0}\right)$ seja positiva definida, então segue que

$$
D g\left(x_{0}, 0\right)=D f\left(x_{0}\right)=0
$$

$\mathrm{e}$

$$
D_{x x} g\left(x_{0}, 0\right)
$$

é positiva definida. Assim, pelo teorema da função implícita, para todo $\lambda$ em uma vizinhança de 0 , existe um único mínimo de $g$, digamos $x_{\lambda}$ em uma vizinhança de $x_{0}$.

Na figura 5.5 vemos o gráfico de $f(x)=x^{2}$ junto com a família $g(x, \lambda)=x^{2}+\lambda x$, para alguns valores de $\lambda$. De fato, $x_{0}=0$ é um ponto de mínimo de $f$ e

$$
D g(x, \lambda)=[2 x+\lambda \quad x]
$$




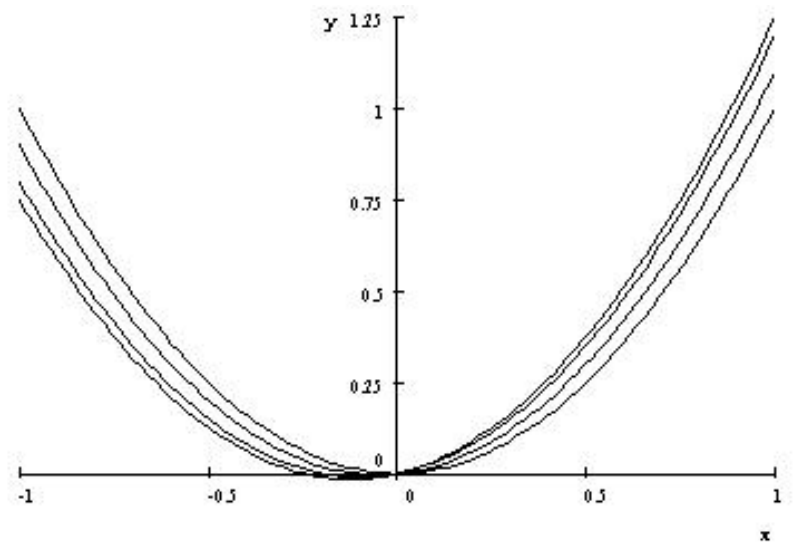

Figura 5.5: Estabilidade do minimizante

ou seja,

$$
\operatorname{Dg}(0,0)=0
$$

e $D_{x x} g=2$. Vemos pelo gráfico que os pontos de mínimo desta família se encontram todos em alguma proximidade da origem, ou seja, do ponto de mínimo da função original.

Brevemente, dado um mínimo de $f$, ao perturbarmos esta função, ainda temos um ponto de mínimo da função perturbada em alguma vizinhança do ponto de mínimo original. Isto indica que o extremo da função tem uma propriedade de estabilidade. No entanto, esta conclusão no caso discutido acima dependeu de hipóteses muito restritivas sobre a regularidade de $f$ e de $h$, bem como sobre a matriz Hessiana de $f$. Uma pergunta natural recai sobre a estabilidade de extremos de uma dada função em contextos mais gerais. É neste sentido que surge a próxima proposição.

Proposição 8 Sejam $\Omega$ um aberto de $\mathbb{R}^{n}, \phi \in \mathcal{C}(\Omega \times(0, T])$ e um ponto $x_{0} \in \Omega$ tal que

$$
\phi\left(x_{0}, T\right)>\phi(x, t) \quad \forall(x, t) \in \mathcal{B}_{\delta}\left(x_{0}, T\right)
$$

para algum $\delta>0$. Então, para todo $r>0$, existe $0<\varepsilon_{r}<r$ tal que a aplicação $\psi_{\varepsilon_{r}}: \Omega \times(0, T) \longrightarrow \mathbb{R}$ dada por

$$
\psi_{\varepsilon_{r}}(x, t)=\phi(x, t)-\frac{\varepsilon_{r}}{T-t}
$$

tem um ponto de máximo local chamado $\left(x_{\varepsilon_{r}}, t_{\varepsilon_{r}}\right)$ tal que $\left\|x_{0}-x_{\varepsilon_{r}}\right\|<r$ e $\left|T-t_{\varepsilon_{r}}\right|<r$.

Demonstração. Fixe $r>0$. Tome um número $0<\rho<r$ e defina o conjunto

$$
A_{r}=\left\{(x, t) \in \Omega \times(0, T) \mid\left\|x-x_{0}\right\| \leq \rho \text { e }|T-t| \leq \rho\right\} .
$$

Claramente, $A_{r}$ é um conjunto compacto. Fixe então $0<\xi<\rho$

Logo, a aplicação

$$
\psi_{\xi}(x, t)=\phi(x, t)-\frac{\xi}{T-t}
$$


tem um ponto de máximo em $A_{r}$. Denotamos este ponto de máximo por $\left(x_{\xi}, t_{\xi}\right)$. É claro que $\left\|x_{0}-x_{x i}\right\|<r$ e $\left|T-t_{x i}\right|<r$. Naturalmente, colocamos $\xi=\varepsilon_{r}$ e concluímos a demonstração.

Se for necessário estimar uma distância entre os máximos de $\psi_{\varepsilon_{r}}$ e $\phi$, podemos tomar $\xi=\frac{\rho}{n}$ para $n \in \mathbb{N}$ apropriado.

Proposição 9 Seja $\Omega$ um aberto de $\mathbb{R}^{n}$ e considere as funções $F \in \mathcal{C}\left((0, T] \times \Omega \times \mathbb{R} \times \mathbb{R}^{n}\right)$, $u \in \mathcal{C}(\Omega \times(0, T])$ e $\phi \in \mathcal{C}^{1}(\Omega \times(0, T])$.

1. Se u é uma solução de viscosidade de

$$
u_{t}+F(t, x, u, D u) \leq 0
$$

em $\Omega \times(0, T], e\left(x_{0}, T\right)$ é um ponto de máximo local de $u-\phi$ em $\Omega \times(0, T]$, então,

$$
\phi_{t}+F\left(x_{0}, T, u\left(x_{0}, T\right), D_{x} \phi\left(x_{0}, T\right)\right) \leq 0 .
$$

2. Se u é uma solução de viscosidade de

$$
u_{t}+F(t, x, u, D u) \geq 0
$$

em $\Omega \times(0, T]$, e $\left(x_{0}, T\right)$ é um ponto de mínimo local de $u-\phi$ em $\Omega \times(0, T]$, então,

$$
\phi_{t}+F\left(x_{0}, T, u\left(x_{0}, T\right), D_{x} \phi\left(x_{0}, T\right)\right) \geq 0 .
$$

Demonstração. Para o item 2, basta utilizarmos 1, a definição de solução viscosa e a proposição 6 . Vejamos então 1.

Tomemos $u$ e $\left(x_{0}, T\right)$ como nas hipóteses do item 1. Vamos então tomar uma aplicação $\psi$ de classe $\mathcal{C}^{\infty}\left(\mathbb{R}^{n+1}\right)$ tal que

$$
0 \leq \psi<1
$$

em todos os pontos de

$$
\mathbb{R}^{n+1} \backslash\left\{\left(x_{0}, T\right)\right\}
$$

com

$$
\psi\left(x_{0}, T\right)=1 .
$$

Note que, por ser um ponto de máximo, e não ser um ponto da fronteira do domínio de $\psi$, $\left(x_{0}, T\right)$ é um ponto crítico desta aplicação. Logo,

$$
D \psi\left(x_{0}, T\right)=0
$$

Observe ainda que $u-(\phi-\psi)$ tem um ponto de máximo em $\left(x_{0}, T\right)$ e, da observação anterior, $D(\phi-\psi)\left(x_{0}, T\right)=D \phi\left(x_{0}, T\right)$. 
Seja $n \in \mathbb{N}$. Defina então $r_{n}=\frac{1}{n}$. Da proposição 8 , existe $\varepsilon_{n}<\frac{1}{n}$ e um ponto $\left(x_{n}, t_{n}\right)$ com

$$
\left\|x_{n}-x_{0}\right\|<\frac{1}{n} \quad e \quad\left|t_{n}-T\right|<\frac{1}{n}
$$

que é máximo local de

$$
\Psi_{n}(x, t)=u(x, t)-\left[(\phi-\psi)(x, t)-\frac{\varepsilon_{n}}{T-t}\right]
$$

É fácil ver que

$$
\frac{\partial}{\partial t} \Psi_{n}(x, t)=\left(\phi_{t}-\psi_{t}\right)(x, t)+\frac{\varepsilon_{n}}{(T-t)^{2}}
$$

e

$$
\frac{\varepsilon_{n}}{(T-t)^{2}}>0
$$

Da hipótese de $u$ ser solução de viscosidade de $u_{t}+F(t, x, u, D u) \leq 0 \mathrm{em} \Omega \times(0, T)$, de 5.4 e de 5.5 obtemos

$$
(\phi-\psi)\left(x_{n}, t_{n}\right)+\frac{\varepsilon_{n}}{(T-t)^{2}}+F\left(x_{n}, t_{n}, u\left(x_{n}, t_{n}\right), D_{x}(\phi-\psi)\left(x_{n}, t_{n}\right)\right) \leq 0,
$$

i.e.

$$
(\phi-\psi)\left(x_{n}, t_{n}\right)+F\left(x_{n}, t_{n}, u\left(x_{n}, t_{n}\right), D_{x}(\phi-\psi)\left(x_{n}, t_{n}\right)\right) \leq 0 .
$$

Defina

$$
G(x, t)=(\phi-\psi)\left(x_{n}, t_{n}\right)+F\left(x_{n}, t_{n}, u\left(x_{n}, t_{n}\right), D_{x}(\phi-\psi)\left(x_{n}, t_{n}\right)\right) .
$$

Por serem $u, F, \phi, \psi, D \phi$ e $D \psi$ funções contínuas, segue que $G$ é uma função contínua. Como

$$
G\left(x_{n}, t_{n}\right) \leq 0
$$

para todo $n \in \mathbb{N}$, temos que

$$
0 \geq \lim _{n \longrightarrow \infty} G\left(x_{n}, t_{n}\right)=G\left(\lim _{n \longrightarrow \infty} x_{n}, \lim _{n \longrightarrow \infty} t_{n}\right)=G\left(x_{0}, T\right)
$$

de onde segue que

$$
G\left(x_{0}, T\right)=\phi_{t}\left(x_{0}, T\right)+F\left(T, x_{0}, u\left(x_{0}, T\right), D_{x} \phi\left(x_{0}, T\right)\right) \leq 0,
$$

e verifica-se o resultado.

Dedicamos o restante desta subseção a alguns resultados acerca dos super e sub-diferenciais de 
funções contínuas e seus exemplos. Alguns destes resultados, apesar de contra-intuitivos a princípio, são elementos importantes na demonstração de fatos que lançam luz a aspectos menos evidentes por trás da definição de solução viscosa. Vejamos alguns deles.

Lema 5 Considere um aberto $\Omega$ de $\mathbb{R}^{n}$ e duas funções contínuas sobre $\Omega$, digamos $f$ e $g$, a valores reais. Então:

1. se $x_{0}$ é um ponto de máximo local de $(f-g)$, tem-se $D^{+} g\left(x_{0}\right) \subset D^{+} f\left(x_{0}\right)$ e $D^{-} f\left(x_{0}\right) \subset$ $D^{-} g\left(x_{0}\right)$.

2. se $x_{0}$ é ponto de máximo de $f$ então $0 \in D^{+} f\left(x_{0}\right)$.

Demonstração. A princípio, usaremos o item 1 e obteremos o 2. Em seguida provaremos o item 1. Seja $g \equiv 0$. Então, $0 \in D^{+} g\left(x_{0}\right)$. Por ser $x_{0}$ um ponto de máximo de $f-g \equiv f-0 \equiv f$, segue do item 1 que $0 \in D^{+} g\left(x_{0}\right) \subset D^{+} f\left(x_{0}\right)$, se onde o resultado segue. Vejamos agora como estabelecer o item 1.

Tomemos $x_{0}$ um ponto de máximo local de $f-g$. Então $(f-g)(x) \leq(f-g)\left(x_{0}\right)$ em alguma vizinhança de $x_{0}$. Como $\left\|x-x_{0}\right\|$ é sempre um número positivo e diferente de zero toda vez que $x \neq x_{0}$, temos que se $x \neq x_{0}$ e se $x$ pertence a esta vizinhança,

$$
\frac{f(x)-f\left(x_{0}\right)-p\left(x-x_{0}\right)}{\left\|x-x_{0}\right\|} \leq \frac{g(x)-g\left(x_{0}\right)-p\left(x-x_{0}\right)}{\left\|x-x_{0}\right\|}
$$

para todo $p \in \mathbb{R}^{n}$. Tomando os limites superiores e inferiores, segue o resultado.

Um fato que evidencia a relação entre os super e sub-diferenciais com o diferencial de uma função - quando este existe - é o seguinte resultado:

Lema 6 Sejam $\Omega$ um aberto de $\mathbb{R}^{n}, x \in \Omega$ e u uma função contínua sobre $\Omega$ a valores reais. Então $u$ é diferenciável em $x$ e $D u(x)=p$ se, e somente se, $D^{+} u(x)=D^{-} u(x)=\{p\}$.

Demonstração. Suponha, a princípio, que $D^{+} u(x)=D^{-} u(x)=\{p\}$. Então temos que

$$
\limsup _{y \rightarrow x} \frac{u(y)-u(x)-p(y-x)}{\|y-x\|}=\liminf _{y \rightarrow x} \frac{u(y)-u(x)-p(y-x)}{\|y-x\|}
$$

o que implica que existe o limite

$$
\lim _{y \rightarrow x} \frac{u(y)-u(x)-p(y-x)}{\|y-x\|}=0
$$

ou seja, $u$ é diferenciável e $D u(x)=p$.

Por outro lado, assuma que $u$ é diferenciável em $x \in \Omega$. Isto implica, novamente pela definição da aplicação derivada, que

$$
\lim _{y \rightarrow x} \frac{u(y)-u(x)-D u(x)(y-x)}{\|y-x\|}=0 .
$$


Como $D u(x)$ é um ponto de $\mathbb{R}^{n}$ temos que $D u(x) \in D^{+} u(x)$ e $D u(x) \in D^{-} u(x)$. Portanto, o conjunto $\{D u(x)\}$ está contido tanto em $D^{+} u(x)$ como em $D^{-} u(x)$.

Tomemos agora um ponto $p \in D^{+} u(x)$. Claramente, existe uma função $\psi$ de classe $\mathcal{C}^{1}$ sobre $\Omega$ tal que $x$ é um ponto de máximo local de $(u-\psi)$ e com $D \psi(x)=p$. Da condição de otimalidade e da diferenciabilidade de $u$, segue que $D u(x)=D \psi(x)=p$ e, portanto, o conjunto $D^{+} u(x)$ está contido em $D u(x)$, implicando na igualdade $D^{+} u(x)=D u(x)$.

Utilizando o fato já demonstrado de que $D^{-} u(x)=-D^{+}(-u)(x)$ e aplicando o argumento anterior a $-u$ obtemos que $D^{-} u(x)=D(u)(x)$, o que conclui a demonstração.

Este resultado tem um interessante significado acerca da noção de solução viscosa de uma equação diferencial. Na verdade ele afirma que se uma solução viscosa for diferenciável, então ela será uma solução clássica.

Para vermos isto basta notarmos que se $u$ for diferenciável, a cardinalidade de seus sub e superdiferenciais será 1 em todo ponto - precisamente, será o diferencial da função naquele ponto. Além disso, por ser $D u(x) \in D^{+} u(x)$ existe uma função $\phi$ de classe $\mathcal{C}^{1}$ tal que $x$ é ponto de máximo de $u-\phi$ e com $D \phi(x)=D u(x)$. Como $x$ é arbitrário, obtemos $F(x, u(x), D u(x)) \leq 0$.

Analogamente, por ser $D u(x) \in D^{-} u(x)$ para todo $x$ em $\Omega$, deve existir $\psi$ continuamente diferenciável sobre $\Omega$ tal que $u-\psi$ tenha um ponto de mínimo local em $x$ e $D \psi(x)=D u(x)$. Aqui obtemos que $F(x, u(x), D u(x)) \geq 0$. Assim, por ser $F(x, u(x), D u(x))=0$ segue que $u$ é uma solução no sentido clássico do problema $F=0$.

Esta constatação engendra uma noção de consistência no conceito de solução viscosa - na verdade sugere que o uso de semi-diferenciais apenas visa contornar o problema da diferenciabilidade em alguns pontos - na medida em que revela a fraqueza desta classe de soluções ao revelar os elementos $p \in D^{+} u(x)$ e $q \in D^{-} u(x)$ como alternativas fracas à derivada clássica de $u$.

Considere agora um aberto $\Omega$ de $\mathbb{R}^{n}$ e uma função contínua $u$ definida sobre este aberto tomando valores reais. Uma proposição curiosa - e que não tem nenhuma razão intuitiva para ser verdadeira - é que, se o super-diferencial e o sub-diferencial de $u$ forem não vazios num mesmo ponto $x$ arbitrário de $\Omega$, então $u$ será diferenciável neste ponto, com $D u(x)=p$ e $D^{+} u(x)=D^{-} u(x)=\{p\}$. Formalmente:

Proposição 10 Sejam $\Omega \subset \mathbb{R}^{n}$ e $u \in \mathcal{C}(\Omega)$. Então, se $D^{+} u(x) \neq \emptyset$ e $D^{-} u(x) \neq \emptyset$ em algum $x \in \Omega$, temos que u é diferenciável em $x, D u(x)=p$ e $D^{+} u(x)=D^{-} u(x)=\{p\}$.

Demonstração. Fixemos $x \in \Omega$ e tomemos um ponto $p \in D^{+} u(x)$. Sabemos que existe uma função $\phi \in \mathcal{C}^{1}(\Omega)$ com $D \phi(x)=p$ tal que $(u-\phi)$ tem um ponto de máximo em $x$. Como já vimos anteriormente, isto implica que $D^{-} u(x) \subset D^{-} \phi(x)$. No entanto, por ser $\phi$ diferenciável, sabemos que $D^{-} \phi(x)=D \phi(x)=p$. Assim, $D^{-} u(x) \subset\{p\}$. Ora, se assumirmos que $D^{-} u(x) \neq \emptyset$, resta-nos apenas que $D^{-} u(x)=\{p\}$.

Suponha que $D^{-} u(x)$ seja diferente do vazio e que $D^{+} u(x)$ tenha mais de um elemento, por exemplo, $p$ e $q$. Então $D^{-} u(x)=\{p\}$ e $D^{-} u(x)=\{q\}$, o que é possível apenas se $p=q$. Logo, se ambos os semi-diferenciais de $u$ em $x$ forem não vazios, eles terão necessariamente o mesmo único 
elemento, digamos $p$. Mas já vimos que isto implica que $u$ é diferenciável no ponto $x$ e $D u(x)=p$, o que conclui nossa demonstração.

Para finalizar nossa discussão acerca dos semi-diferenciais ${ }^{3}$ aqui utilizados vamos expor um resultado que nos permitirá obter super e sub-diferenciais de funções a partir do simples cálculo de limites laterais. Vejamos.

Lema 7 Sejam $\Omega \subset \mathbb{R}$, aberto, e $u \in \mathcal{C}(\Omega)$. Considere um ponto $x_{0} \in \Omega$ e suponha que

$$
\alpha_{1}=\lim _{x \rightarrow x_{0}^{+}} \frac{u(x)-u\left(x_{0}\right)}{x-x_{0}}
$$

$e$

$$
\alpha_{2}=\lim _{x \rightarrow x_{0}^{-}} \frac{u(x)-u\left(x_{0}\right)}{x-x_{0}} .
$$

Então, se $\alpha_{1} \leq \alpha_{2}$ temos que $D^{+} u\left(x_{0}\right)=\left[\alpha_{1}, \alpha_{2}\right]$ e se $\alpha_{2} \leq \alpha_{1}$ temos que $D^{-} u\left(x_{0}\right)=\left[\alpha_{2}, \alpha_{1}\right]$.

Demonstração. Suponhamos inicialmente que $\alpha_{1} \leq \alpha_{2}$ e que $p \in D^{+} u\left(x_{0}\right)$. Então, sabemos que existe uma aplicação $\psi \in \mathcal{C}^{1}(\Omega)$ tal que $\psi^{\prime}\left(x_{0}\right)=p$ e $u-\psi$ tem um ponto de máximo local em $x_{0}$. Como já foi utilizado anteriormente, isto implica que

$$
\frac{u(x)-u\left(x_{0}\right)}{\left\|x-x_{0}\right\|} \leq \frac{\psi(x)-\psi\left(x_{0}\right)}{\| x-x_{0} \mid}
$$

em tal vizinhança. Suponhamos que tal vizinhança seja da forma $] x_{0}-\varepsilon, x_{0}+\varepsilon[$. Então, é fácil vermos que

$$
\lim _{x \rightarrow x_{0}^{+}} \frac{u(x)-u\left(x_{0}\right)}{x-x_{0}} \leq \lim _{x \rightarrow x_{0}} \frac{\psi(x)-\psi\left(x_{0}\right)}{x-x_{0}} \leq \lim _{x \rightarrow x_{0}^{-}} \frac{u(x)-u\left(x_{0}\right)}{x-x_{0}}
$$

o que implica

$$
\alpha_{1} \leq \psi^{\prime}\left(x_{0}\right) \leq \alpha_{2}
$$

Assim, obtemos que $D^{+} u\left(x_{0}\right) \subset\left[\alpha_{1}, \alpha_{2}\right]$. Basta provarmos a inclusão contrária.

Para isto, suponhamos, a princípio, que $\alpha_{1}=\alpha_{2}=\alpha^{*}$. Então, $u$ é diferenciável em $x_{0}$ e, assim, $D^{+} u\left(x_{0}\right)$ tem um único elemento, precisamente, $\alpha^{*}$. Logo, segue que $D^{+} u\left(x_{0}\right)=\alpha^{*}$, e a demonstração está concluída.

Suponhamos então que $\alpha_{1}<\alpha_{2}$ e tomemos $\left.p \in\right] \alpha_{1}, \alpha_{2}[$. Assim, existem $r$ e $s$, estritamente positivos, tais que

$$
\frac{u(x)-u\left(x_{0}\right)}{x-x_{0}} \leq p
$$

para todo $x \mathrm{em}] x_{0}, x_{0}+r[\mathrm{e}$

$$
\frac{u(x)-u\left(x_{0}\right)}{x-x_{0}} \geq p
$$

\footnotetext{
${ }^{3}$ Mencionamos aqui, para efeito de ilustração, que no caso de uma função $f: \mathbb{R}^{n} \longrightarrow \mathbb{R}$ convexa, seu sub-diferencial no ponto $x \in \mathbb{R}^{n}$, será o conjunto $\left\{p \in \mathbb{R}^{n} \mid f(y) \geq f(x)+p(y-x) \forall y \in \mathbb{R}^{n}\right\}$. Para detalhes acerca desta definição, ou aplicações na análise de convexidade, sugerimos [26].
} 
para todo $x$ em $] x_{0}-s, x_{0}[$.

Isto implica que, se for $\varepsilon=\min \{r, s\}$, temos que

$$
\frac{u(x)-u\left(x_{0}\right)-p\left(x-x_{0}\right)}{\left|x-x_{0}\right|} \leq 0
$$

para todo $x$ em $] x-\varepsilon, x+\varepsilon[$. Logo,

$$
\limsup _{x \rightarrow x_{0}} \frac{u(x)-u\left(x_{0}\right)-p\left(x-x_{0}\right)}{\left|x-x_{0}\right|} \leq 0,
$$

e, temos que $p \in D^{+} u\left(x_{0}\right)$.

Para a parte da proposição envolvendo $D^{-} u\left(x_{0}\right)$, basta utilizarmos o fato de que $D^{-} u\left(x_{0}\right)=$ $-D^{+}(-u)\left(x_{0}\right)$ e repetirmos a argumentação anterior.

Este resultado nos permite, por exemplo, obter $D^{+}$e $D^{-}$para a aplicação $u(x)=|x|$, não diferenciável na origem. Vejamos:

$$
\lim _{x \rightarrow 0^{+}} \frac{|x|}{x}=1
$$

$\mathrm{e}$

$$
\lim _{x \rightarrow 0^{-}} \frac{|x|}{x}=-1
$$

ou seja, $\alpha_{1}=1$ e $\alpha_{2}=-1$. Assim, temos que $D^{-} u(0)=[-1,1]$. Ora, como já notamos, o fato de $|x|$ não ser diferenciável em $x=0$ implica que $D^{+} u(x)$ deverá ser vazio.

Além disso, se retomarmos nosso exemplo que abre esta subseção, veremos que $1-(p)^{2} \geq 0$ para todo $p$ em $D^{-} u(0)$. Como o super-diferencial é vazio, notamos que $u(x)=|x|$ satisfaz de fato às condições necessárias sobre seus semi-diferenciais para ser uma solução viscosa de $1-\left(u^{\prime}(x)\right)^{2}=0$.

Observemos o que ocorre com uma função diferenciável qualquer, $f: \mathbb{R} \longrightarrow \mathbb{R}$ em um ponto $\bar{x}$ arbitrário do domínio. Em função da diferenciabilidade, $\alpha_{1}=\alpha_{2}=\alpha^{*}$, o que implica que $D^{+} f(\bar{x})=D^{-} f(\bar{x})=f^{\prime}(\bar{x})$ o que já era esperado, em vista do que verificamos nas proposições anteriores.

Para concluir esta seção vamos discutir dois aspectos das soluções de viscosidade que são de ampla familiaridade da teoria das equações diferenciais parciais do tipo elíptica e parabólica, a saber, um princípio do máximo e um princípio de comparação. Este princípio de comparação difere do teorema de comparação que apresentaremos adiante quando estudarmos os aspectos qualitativos de soluções viscosas no contexto das equações de HJ.

Definição 44 Considere um dominio $\Omega \subset \mathbb{R}^{n}$ e uma função contínua sobre $\Omega$ a valores reais. Diremos que u satisfaz o princípio da comparação, $(P C)$, se para qualquer função $\psi$ de classe $\mathcal{C}^{1}(\Omega)$ e para qualquer aberto $\mathcal{A} \subset \Omega, F(x, \psi(x), D \psi(x))>0$ em $\mathcal{A}$ e $u \leq \psi$ em $\partial \mathcal{A}$ implicarem $u-\psi \leq 0$ em $\mathcal{A}$.

Definição 45 Nas condições da definição anterior, dizemos que u satisfaz o princípio do máximo, 
(PM), se para qualquer $\psi$ de classe $\mathcal{C}^{1}(\Omega)$ e $\mathcal{A}$ aberto de $\Omega$, a desigualdade

$$
F(x, \psi(x), D \psi(x))>0
$$

em $\mathcal{A}$ implicar que $u-\psi$ não pode ter um máximo não negativo em $\mathcal{A}$.

Suponha que $u$ satisfaça ao princípio do máximo e suponha ainda que para qualquer função $\psi$ de classe $\mathcal{C}^{1}(\Omega)$ e para qualquer aberto $\mathcal{A} \subset \Omega, F(x, \psi(x), D \psi(x))>0$ em $\mathcal{A}$ e $u \leq \psi$ em $\partial \mathcal{A}$. Então, $(u-\psi) \leq 0$ na fronteira de $\mathcal{A}$. Suponha, por absurdo, que exista um $x_{0}$ em $\mathcal{A}$ tal que $u\left(x_{0}\right)>\psi\left(x_{0}\right)$. Então $(u-\psi)\left(x_{0}\right)>0$, o que contraria o princípio do máximo.

Assim, vemos que o princípio do máximo implica no princípio da comparação. O próximo resultado relaciona estas duas últimas definições com as soluções viscosas. Consideremos o seguinte problema com condições de fronteira:

$$
F(x, u(x), D u(x))=0 \text { em } \mathcal{A}
$$

com

$$
u \equiv \psi \text { em } \partial \mathcal{A}
$$

onde $\psi \in \mathcal{C}^{1}(\mathcal{A})$.

Então segue que:

Proposição 11 Seja $\Omega \in \mathbb{R}^{n}$, aberto, e considere a equação $F(x, u(x), D u(x))=0$ em $\Omega$ com $u=\psi$ em $\partial \Omega$. Se $u$, contínua, satisfaz (PC) então u é uma solução viscosa de $F=0$. Por outro lado, se u é uma solução viscosa de $F=0$ e a aplicação $r \longmapsto F(x, r, p)$ é não decrescente para todo $(x, p) \in \Omega \times \mathbb{R}^{n}$, então u satisfaz $(P M)$ e, portanto, $(P C)$.

Demonstração. Suponhamos que $u$ satisfaz o princípio da comparação, mas, por absurdo, suponhamos ainda que $u$ não seja uma sub-solução viscosa do problema acima.

Por ser $u=\psi$ em $\partial \Omega$ e por $u$ satisfazer o princípio da comparação, temos que $u \leq \psi$ em $\Omega$. Portanto, existe um ponto $x$ tal que $(u-\psi)(x)=0$, o que será um ponto de máximo da aplicação $u-\psi$. Pelo princípio da comparação temos que

$$
F(x, u(x), D \psi(x))>0
$$

Da continuidade de $u-\psi$ temos que, para valores de $n \in \mathbb{N}$ grandes, i.e., a partir de um determinado $n^{*}$,

$$
a_{n}=\sup _{\partial \mathcal{B}_{\frac{1}{n}}(x)}(u-\psi) \leq 0 .
$$

Claro que

$$
u-\psi-a_{m}=u-\psi-\sup _{\partial \mathcal{B}_{\frac{1}{n}}(x)}(u-\psi) \leq 0
$$


em $\partial \mathcal{B}_{\frac{1}{n}}(x)$. Mais ainda, $(u-\psi)(x)-a_{n}=0-a_{n} \geq 0$, uma vez que $a_{n}$ deve ser negativo. Assim, deve existir, para cada $n \in \mathbb{N}$, um ponto - que chamaremos de $x_{n}$ - tal que

$$
F\left(x_{n}, \psi\left(x_{n}\right), D \psi\left(x_{n}\right)\right) \leq 0 .
$$

Pela continuidade de $F$, por ser $a_{n} \longrightarrow 0$ e $x_{n} \longrightarrow x$, temos que

$$
F(x, u(x), D \psi(x)) \leq 0
$$

o que é uma contradição, pois havíamos suposto que $u$ não fosse uma sub-solução viscosa.

Por outro lado, suponha que $u$ é uma sub-solução viscosa do problema considerado e seja $\psi$ uma função continuamente diferenciável sobre $\Omega$ tal que

$$
F(x, \psi(x), D \psi(x))>0 \text { para todo } x \in \Omega \text {. }
$$

Suponha agora que a função $u-\psi$ atinja um máximo positivo em algum ponto $x_{0}$ do domínio. Então, a hipótese de monotonicidade implicaria

$$
0<F\left(x_{0}, \psi\left(x_{0}\right), D \psi\left(x_{0}\right)\right) \leq F\left(x_{0}, u\left(x_{0}\right), D \psi\left(x_{0}\right)\right) \leq 0
$$

o que contradiz a hipótese de que $u$ é sub-solução viscosa. Assim, a função $u-\psi$ não pode ter um máximo não negativo e, com isto, satisfaz o princípio do máximo. Como vimos anteriormente, o princípio do máximo implica no princípio da comparação, e, então, o resultado desejado segue.

Analogamente, podemos obter um resultado para super-soluções viscosas.

\subsection{Um teorema de existência e unicidade}

Nesta seção, serão apresentados um teorema de existência e um teorema de unicidade de soluções viscosas. O primeiro assegurará a existência de solução da equação

$$
u_{t}+H(x, u(x, t), D u(x, t)) \quad \text { em } \quad \Omega \times(0, T)
$$

com as condições iniciais

$$
u \equiv \phi \quad \text { em } \Omega \times\{0\}
$$

onde $\Omega \subset \mathbb{R}^{n}$ é um aberto.

Por sua vez, o teorema de unicidade será apresentado para uma equação de caráter mais geral, a saber

$$
u_{t}+H(x, t, u(x, t), D u(x, t)) \quad e m \quad \Omega \times[0, T]
$$


com as condições de contorno

$$
u \equiv \phi \quad \text { em } \Omega \times\{0\}
$$

onde $\Omega \subset \mathbb{R}^{n}$ é, novamente, um aberto e $\phi$ uma função contínua sobre o domínio.

Apesar de diferirem em seus conteúdos, os teoremas fornecem as condições necessárias ao problema de nosso interesse - a aplicação aos jogos diferenciais - uma vez que a equação que veremos adiante na formulação do jogo diferencial terá o aspecto

$$
u_{t}+H(x, D u(x, t)) \quad \text { em } \Omega \times[0, T]
$$

com as condições de contorno

$$
u \equiv \phi \quad e m \quad \Omega \times\{0\}
$$

Passemos agora à discussão destes resultados.

\subsubsection{Módulos de continuidade}

Definição 46 Considere uma função $m: \mathbb{R}^{+} \longrightarrow \mathbb{R}^{+}$com as seguintes propriedades:

1. $m \in \mathcal{C}\left(\mathbb{R}^{+}\right)$não-decrescente.

2. $m(0)=0$.

3. sub-aditividade: $m(a+b) \leq m(a)+m(b) \forall a, b \in \mathbb{R}^{+}$.

4. $m(r) \leq(r+1) m(1)$ para todo $r \in \mathbb{R}^{+}$.

Então, $m$ será chamada um módulo de continuidade.

$\mathrm{O}$ segundo item da definição é equivalente ao fato de $\lim _{t \rightarrow 0^{+}} m(t)=0$. Isto pela continuidade de $m$. Já o quarto item da definição segue da combinação do primeiro com o terceiro. Vejamos: tome $r$ dado e seja $\bar{r}$ o maior inteiro menor do que $r$. Então, por $1, m(\bar{r}) \leq m(r) \leq m(\bar{r}+1)$ e, por $3,(\bar{r}+1) \leq m(\bar{r})+m(1) \leq(\bar{r}+1) m(1) \leq(r+1) m(1)$. É a partir deste item 4 que podemos concluir que se $m(1)=0$ então $m$ é identicamente nula.

Vejamos alguns exemplos:

Exemplo 9 1. $f: \mathbb{R}^{+} \longrightarrow \mathbb{R}^{+}$dada por $f(x)=x^{2}$ não é um módulo de continuidade. Apesar de ser contínua, e de fixar a origem, esta aplicação é super-aditiva.

2. $g: \mathbb{R}^{+} \longrightarrow \mathbb{R}^{+}$dada por $g(x)=x^{\frac{1}{2}}$ é um módulo de continuidade. Novamente, é claro que $g$ é contínua, não decrescente e que fixa o zero. Verifiquemos a sub-aditividade:

$$
\left(a^{\frac{1}{2}}+b^{\frac{1}{2}}\right)^{2}=a+b+2(a b)^{\frac{1}{2}}>a+b=\left((a+b)^{\frac{1}{2}}\right)^{2} .
$$


3. Toda aplicação linear (de $\mathbb{R}^{+}$em $\mathbb{R}^{+}$) é um módulo de continuidade.

Procuraremos soluções viscosas em um espaço de funções que será definido utilizando a noção de módulo de continuidade.

Definição 47 Seja $f: X \longrightarrow \mathbb{R}$. $f$ será dita uniformemente-módulo-contínua se existirem $r_{f}>0$ e um módulo de continuidade $m_{f}$ tal que

$$
\left|f\left(x_{1}\right)-f\left(x_{2}\right)\right| \leq m_{f}\left(\left|x_{1}-x_{2}\right|\right)
$$

para todo $x_{1}, x_{2} \in X$ com $\left|x_{1}-x_{2}\right| \leq r_{f}$. O espaço das funções uniformemente-módulo-contínuas sobre um domínio $X$ será denotado por $\mathcal{U C}_{m_{x}}(X)$.

Proposição 12 Defina por $\mathcal{U C}(X \times Y)$ o espaço das funções uniformemente contínuas em $X \times Y$. Então,

$$
\mathcal{U C}_{m_{x}}(X \times Y) \subset \mathcal{U C}(X \times Y)
$$

Demonstração. Seja $f \in \mathcal{U C}_{m_{x}}(X \times Y)$. Então, existem $r_{f}>0$ e um módulo de continuidade $m_{f}$ tais que $\left\|f\left(x_{2}, y\right)-f\left(x_{2}, y\right)\right\| \leq m_{f}\left(\left\|x_{1}-x_{2}\right\|\right)$ para todo $x_{1}, x_{2} \in X$ com $\left\|x_{1}-x_{2}\right\| \leq r_{f}$. Seja $\varepsilon \geq 0$. Se for $\varepsilon \geq m_{f}(r)$ então, tomamos $\delta=r$. Se for, por outro lado, $\varepsilon \leq m_{f}(r)$, observamos que, pela definição de módulo de continuidade segue que

$$
\left\|f\left(x_{2}, y\right)-f\left(x_{2}, y\right)\right\| \leq m_{f}\left(\left\|x_{1}-x_{2}\right\|\right) \longrightarrow 0
$$

quando $\left\|x_{1}-x_{2}\right\| \longrightarrow 0$. Logo, para todo $\varepsilon \geq 0$ existe um $\delta \geq 0$ tal que se quaisquer dois pontos de $X$ estão $\delta$-próximos, então $f\left(x_{2}, y\right)$ e $f\left(x_{2}, y\right)$ estão $\varepsilon$-próximos para todo $y \in Y$.

Lema 8 O conjunto dos módulos de continuidade, que denotaremos por $\mathcal{M}$, é um cone sob a soma e produto usual de funções por escalares.

Demonstração. Sejam dois módulos de continuidade $m_{1}$ e $m_{2}$. Definindo a soma de módulos de continuidade como usualmente, i.e., $\left(m_{1}+m_{2}\right)(t)=m_{1}(t)+m_{2}(t)$ então, claramente, $m_{1}+m_{2}$ é contínua, não decrescente, fixa a origem e é sub-aditiva. Seja agora $\lambda$ um escalar real. Se $\lambda<0$, então $\lambda m_{1}$ será super-aditiva. Logo, $\lambda m_{1}$ é módulo de continuidade apenas se for $\lambda \geq 0$.

Lema 9 Seja $m$ um módulo de continuidade.

1. Se existirem $\varepsilon>0, \delta \in[0, m(1)]$ e $r \in[0,1]$ tais que $\frac{r^{2}}{\varepsilon} \leq \delta+m(r)$ então

$$
r \leq 2 \sqrt{m(1) \varepsilon}
$$

$e$

$$
\frac{r^{2}}{\varepsilon} \leq \delta+m(2 \sqrt{m(1) \varepsilon})
$$


2. Sejam $E>0$ e $F>m(1)$ e considere a aplicação $k:(0, \infty) \longrightarrow \mathbb{R}$ definida por

$$
k(\gamma)=\max \left\{m\left(E \gamma r+\left(\frac{r}{F}\right)^{\frac{1}{\gamma}}\right)-r \mid r \in[0, F]\right\} .
$$

Então,

$$
\lim _{\gamma \longrightarrow 0^{+}} k(\gamma)=0
$$

\section{Demonstração.}

1. Para o primeiro item, a monotonicidade de $m$ e 5.12 implicam em 5.13. Provemos então 5.12. Da hipótese temos que

$$
r^{2} \leq \varepsilon(\delta+m(r)) \leq \varepsilon(\delta+(r+1) m(1)) \leq \varepsilon(3 \max \{\delta, r m(1), m(1)\}) \leq 4 \varepsilon m(1)
$$

o que implica $r \leq 2 \sqrt{m(1) \varepsilon}$.

2. Para o segundo item, consideramos a função $f_{\gamma}(r)=m\left(E \gamma r+\left(\frac{r}{F}\right)^{\frac{1}{\gamma}}\right)-r$ definida sobre $[0, F]$. Por ser contínua e estar definida sobre um compacto, claramente $f_{\gamma}$ possui um ponto de máximo, digamos $r_{\gamma}$. Vemos que $f_{\gamma}\left(r_{\gamma}\right) \geq f_{\gamma}(0)=m(0)-0=0$. Isto implica que em seu ponto de máximo, $f_{\gamma}$ é maior ou igual que zero. Para demonstrarmos o item 2 do Lema, mostraremos que

$$
\lim _{\gamma \longrightarrow 0} f_{\gamma}\left(r_{\gamma}\right)=0
$$

Observamos que

$$
f_{\gamma}\left(r_{\gamma}\right) \leq f_{\gamma}\left(r_{\gamma}\right)+r_{\gamma} \leq m\left(E \gamma r_{\gamma}+\left(\frac{r_{\gamma}}{F}\right)^{\frac{1}{\gamma}}\right)
$$

Assim, como $0 \leq f_{\gamma}\left(r_{\gamma}\right) \leq m\left(E \gamma r_{\gamma}+\left(\frac{r_{\gamma}}{F}\right)^{\frac{1}{\gamma}}\right)$, para concluirmos nossa demonstração, basta mostrarmos que - uma vez que $m$ é contínua - $E \gamma r_{\gamma}+\left(\frac{r_{\gamma}}{F}\right)^{\frac{1}{\gamma}} \longrightarrow 0$. Como $r_{\gamma} \in[0, F]$, $E$ é fixo e $\gamma$ tende a zero, o primeiro termo tende a zero. Resta então mostrarmos que $\left(\frac{r_{\gamma}}{F}\right)^{\frac{1}{\gamma}}$ também vai para zero quando $\gamma$ vai para zero. Com esta finalidade, tomemos uma seqüência $\left(\gamma_{n}\right)_{n \in \mathbb{N}}$ tal que $\gamma_{n} \longrightarrow 0$. Seja então $\rho_{n}=r_{\gamma_{n}}$.

Provaremos que existe $\rho \in] 0, F\left[\right.$ tal que $0 \leq \rho_{n} \leq \rho \leq F$ para todo natural $n$. Suponhamos, por absurdo, que não exista tal $\rho$. Então existe uma subseqüência $\rho_{n_{k}}$ tal que $\rho_{n_{k}} \longrightarrow F$, quando $k \longrightarrow \infty$, o que implica

$$
0 \leq \lim _{k \longrightarrow \infty} f_{\gamma_{n_{k}}}\left(\rho_{n_{k}}\right)=m(1)-F<0
$$

um absurdo em vista de $f_{\gamma}\left(r_{\gamma}\right) \geq 0$.

Assim, temos que

$$
0 \leq\left(\frac{r_{\gamma_{n}}}{F}\right)^{\frac{1}{\gamma_{n}}} \leq\left(\frac{\rho}{F}\right)^{\frac{1}{\gamma_{n}}}<1 .
$$


Desta maneira,

$$
\limsup _{n \rightarrow \infty}\left(\frac{\rho_{n}}{F}\right)^{\frac{1}{\gamma_{n}}} \leq 1
$$

de onde segue o resultado.

Proposição 13 Sejam $\Omega \subset \mathbb{R}^{n}$, aberto, com $\partial \Omega \neq \emptyset, T>0$ e u,v $\in \mathcal{U C}_{m_{x}}(\bar{\Omega} \times[0, T])$. Defina $d: \bar{\Omega} \longrightarrow \mathbb{R}$ por $d(z)=\operatorname{dist}(z, \partial \Omega)$. Suponha que $u \leq v$ em $\partial \Omega \times[0, T]$. Então existem $r>0$ e um módulo de continuidade, não identicamente nulo, tal que

1.

$$
|u(x, t)-u(y, t)|+|v(x, t)-v(y, t)| \leq m(\|x-y\|)
$$

para todo $(x, y, t) \in \bar{\Omega} \times \bar{\Omega} \times[0, T]$ tal que $\|x-y\| \leq r$,

2.

$$
u(x, t)-v(y, t) \leq m(\|x-y\|)+m(\min d(x), d(y))
$$

para todo $(x, y, t) \in \bar{\Omega} \times \bar{\Omega} \times[0, T]$ tal que $\|x-y\| \leq r$ e $\min \{d(x), d(y)\} \leq r$.

\section{Demonstração.}

1. Sejam $u, v \in \mathcal{U C}_{m_{x}}(\bar{\Omega} \times[0, T])$ existem $m_{u}$ e $m_{v}$, módulos de continuidade, e escalares $r_{u}$ e $r_{v}$ estritamente maiores do que zero, tais que

$$
|u(x, t)-u(y, t)| \leq m_{u}(\|x-y\|)
$$

para todo $(x, y, t) \in \bar{\Omega} \times \bar{\Omega} \times[0, T]$ tal que $\|x-y\| \leq r_{u} \mathrm{e}$

$$
|v(x, t)-v(y, t)| \leq m_{v}(\|x-y\|)
$$

para todo $(x, y, t) \in \bar{\Omega} \times \bar{\Omega} \times[0, T]$ tal que $\|x-y\| \leq r_{v}$. Como vimos anteriormente, $\mathcal{M}$ é um cone. E assim, $m_{u}+m_{v}=m$ é um módulo de continuidade. Se tomarmos $r=\min \left\{r_{u}, r_{v}\right\}$ então segue que, para todo $(x, y, t) \in \bar{\Omega} \times \bar{\Omega} \times[0, T]$ tal que $\|x-y\| \leq r$ temos

$$
|u(x, t)-u(y, t)|+|v(x, t)-v(y, t)| \leq m_{u}(\|x-y\|)+m_{v}(\|x-y\|)=m(\|x-y\|),
$$

de onde segue o resultado para 1.

2. Sem perda de generalidade, podemos supor que $\min \{d(x), d(y)\}=d(x)$ - pois se for o contrário, basta trocarmos o nome dos pontos. Assim sendo, temos

$$
d(x)=\inf \{\|x-a\| \mid a \in \partial \Omega\}
$$


e, pela definição de ínfimo, existe uma seqüência $\left(a_{n}\right)_{n \in \mathbb{N}}$, em $\partial \Omega$ tal que $\left\|x-a_{n}\right\| \longrightarrow d(x)$. Por um lado, $\left\|a_{n}\right\| \leq\left\|x-a_{n}\right\|+\|x\| \leq d(x)+\|x\|+1$, o que implica que esta seqüência é limitada, e, portanto, tem subseqüência convergente. Suponha que $a_{n} \longrightarrow \bar{a}$. Por outro lado, $a_{n} \in \partial \Omega$ para todo $n \in \mathbb{N}$. Como este conjunto é fechado, $\bar{a} \in \partial \Omega$. Assim, pela continuidade da norma, $\left\|x-a_{n}\right\| \longrightarrow\|x-\bar{a}\|$. Já, pela unicidade do limite, $\|x-\bar{a}\|=d(x)$.

Com isto, encontramos um ponto na fronteira do domínio que realiza a distância de $x$ a esta fronteira. Segue que

$$
\begin{aligned}
u(x, t) & -v(y, t)=u(x, t)-u(\bar{a}, t)-v(y, t)+u(\bar{a}, t) \\
& \leq u(x, t)-u(\bar{a}, t)-v(y, t)+v(\bar{a}, t) \\
& \leq|u(x, t)-u(\bar{a}, t)|+|v(y, t)-v(\bar{a}, t)| \\
& \leq|u(x, t)-u(y, t)|+|u(y, t)-u(\bar{a}, t)|+|v(y, t)-v(\bar{a}, t)| \\
& \leq m_{u}(\|x-y\|)+m(\|y-\bar{a}\|) \\
& \leq m(\|x-y\|)+m(d(x)) \\
=m(\|x-y\|)+m(\min \{d(x), d(y)\}), \quad &
\end{aligned}
$$

o que conclui o resultado.

\subsubsection{Um teorema de comparação}

Nesta subseção será enunciado um teorema de comparação - do tipo do princípio do máximo para as soluções viscosas da equação de evolução de Hamilton-Jacobi. Consideremos inicialmente o seguinte problema de Cauchy:

$$
u_{t}+H\left(x, t, u(x, t), D_{x} u(x, t)\right)=0
$$

em $\Omega \times[0, T)$, com a condição inicial

$$
u(x, 0)=\phi(x)
$$

Vamos discutir as condições sob as quais, caso uma solução de viscosidade exista para este problema - lembrando que procuraremos por soluções de viscosidade no espaço de funções $\mathcal{U C}_{m_{x}}(\bar{\Omega} \times$ $[0, T])$ - esta será única. As condições de existência serão discutidas na próxima subseção.

Basicamente, as hipóteses do teorema de comparação que enunciaremos envolvem o Hamiltoniano $H$, presente na equação HJ, e algumas de suas condições de regularidade. Vejamos:

Condição $1 \quad$ 1. $H \in \mathcal{C}\left(\Omega \times[0, T] \times \mathbb{R} \times \mathbb{R}^{n}\right)$ 
2. $\forall R>0 \exists m_{R} \in \mathcal{M}$ tal que

$$
|H(x, t, r, p)-H(y, t, r, q)| \leq m_{R}(\|x-y\|+\|p-q\|)
$$

para todo $(x, t, r, p),(y, t, r, q) \in \Omega \times[0, T] \times \mathbb{R} \times \overline{\mathcal{B}_{R}(0)}$

3. a aplicação $r \longmapsto H(x, t, r, p)$ é não decrescente para todo $(x, t, p) \in \Omega \times[0, T] \times \mathbb{R}^{n}$

4. $\exists m_{H} \in \mathcal{M}$ tal que $\forall(t, r) \in[0, T] \times \mathbb{R},(x, y) \in \Omega \times \Omega$ e $\lambda \geq 0$, temos que

$$
H(x, t, r, \lambda(x-y))-H(y, t, r, \lambda(x-y)) \geq-m_{H}\left(\lambda\|x-y\|^{2}+\|x-y\|\right) .
$$

O primeiro e o terceiro item da condição 1 são claros. Já o segundo e o quarto referem-se a alguma regularidade mais forte do que apenas a continuidade e não tão rigorosa como a continuidade uniforme.

A seguir, enunciaremos o teorema da comparação e obteremos a unicidade de soluções como sua consequiência. O restante da seção será dedicado à demonstração do teorema.

Teorema 12 (Teorema de Comparação) Considere o problema de Cauchy dado por 5.14 e 5.15 e suponha válida a condição 1. Sejam $u, v \in \mathcal{U C}_{m_{x}}(\bar{\Omega} \times[0, T])$ tais que u é sub-solução viscosa de 5.14 e 5.15 e $v$ é super-solução viscosa de 5.14 e 5.15. Suponha ainda que $u \leq v$ em $\Omega \times\{0\}$ e $u \leq v$ em $\partial \Omega \times[0, T]$. Então, $u \leq v$ em $\Omega \times[0, T]$.

Corolário 3 Suponha válida a condição 1 e considere $u_{1}, u_{2} \in \mathcal{U C}_{m_{x}}(\bar{\Omega} \times[0, T])$, soluções viscosas de 5.14 e 5.15. Então, se $u_{1}=u_{2}$ em $\partial \Omega \times[0, T], u_{1}=u_{2}$ em $\Omega \times[0, T]$.

Demonstração. Primeiramente, observe que tanto $u_{1}$, quanto $u_{2}$, são, ao mesmo tempo, subsoluções e super-soluções viscosa do Problema de Cauchy em questão. Como ambas são iguais a $\phi$ em $t=0$ e, por hipótese, também são iguais em $\partial \Omega \times[0, T]$, segue que $u_{1} \leq u_{2}$ e $u_{2} \leq u_{1}$.

Resta-nos agora expor a demonstração do teorema de comparação. Para tanto, vamos estudar alguns lemas. Nestes lemas, ao considerarmos abertos $\Omega$ de $\mathbb{R}^{n}$, imporemos a condição de que $\partial \Omega \neq \emptyset$. Esta condição apenas significa que existem pontos na fronteira de $\Omega$ que não estão incluídos no conjunto.

Lema 10 Sejam $\Omega \in \mathbb{R}^{n}$ com $\partial \Omega \neq \emptyset, T>0$ fixo, $H, G \in \mathcal{C}\left(\Omega \times[0, T] \times \mathbb{R} \times \mathbb{R}^{n}\right)$ e $u, v \in$ $\mathcal{C}(\Omega \times] 0, T[)$. Suponha que u é uma sub-solução viscosa de

$$
u_{t}+H\left(x, t, u, D_{x} u\right)=0
$$

em $\Omega \times] 0, T[$ e $v$ é super-solução viscosa de

$$
v_{t}+H\left(y, t, v, D_{y} v\right)=0
$$

em $\Omega \times] 0, T[$. 
Então, a função definida por $w=u-v$ é solução viscosa de

$$
w_{t}+H\left(x, t, u, D_{x} u\right)-G\left(y, t, v,-D_{y} v\right) \leq 0 \quad \text { em } \quad \Omega \times \Omega \times[0, T] .
$$

Demonstração. Da hipótese e das definições de sub e super-solução viscosa, obtemos que $u_{t}+$ $H\left(x, t, u, D_{x} u\right) \leq 0$ e $v_{t}+G\left(y, t, v, D_{y} v\right) \geq 0$, ambas no sentido da viscosidade. Assim sendo, é claro que se estivéssemos lidando com soluções clássicas bastaria mostrarmos que a função $w$ definida no Lema satisfaz a desigualdade.

Porém, em se tratando de soluções de viscosidade, não basta apenas verificarmos que a função $w(x, y, t)=u(x, t)-v(y, t)$ satisfaz à desigualdade implicada. Precisamos sim demonstrar que, se $\psi$ é uma função de classe $\mathcal{C}^{1}$, e $(a, b, c)$ é um ponto de máximo local de $(w-\psi)$, então vale

$$
\psi_{t}(a, b, c)+H\left(a, c, u(a, c), D_{x} \psi(a, b, c)\right)-G\left(b, t, v(b, t),-D_{y} \psi(a, b, c)\right) \leq 0 .
$$

Para tanto, tomemos uma função $\psi$ de classe $\mathcal{C}^{1}$ e consideremos $(a, b, c)$, um ponto de máximo local. Suporemos a princípio que este é de fato um ponto de máximo local estrito. Seja $\bar{r}>0$ tal que a vizinhança $\left.V_{(a, b, c)}=\mathcal{B}_{\bar{r}}(a) \times \mathcal{B}_{\bar{r}}(b) \times\right] c-\bar{r}, c+\bar{r}[$ está contida em $\Omega \times \Omega \times] 0, T[\mathrm{e}$

$$
(w-\psi)(a, b, c)>(w-\psi)(x, y, t)
$$

$\forall(x, y, t) \in V_{(a, b, c)}$.

Defina a função $d: \Omega \times \Omega \times[0, T] \times[0, t] \longrightarrow \mathbb{R}$ como

$$
d(x, y, t, s)=\max \{\|x-a\|,\|y-b\|,\|t-c\|,\|s-c\|\} .
$$

Vemos que, para todo $r$, existe $\varepsilon=\varepsilon(r)$ tal que a aplicação $\theta: \Omega \times \Omega \times] 0, T[\times] 0, t[\longrightarrow \mathbb{R}$ dada por

$$
\theta(x, y, t, s)=u(x, t)-v(y, s)-\psi(x, y, t)-\frac{(t-s)^{2}}{\varepsilon}
$$

tem um ponto de máximo local $\left(x_{r}, y_{r}, t_{r}, s_{r}\right) \operatorname{com} d\left(x_{r}, y_{r}, t_{r}, s_{r}\right) \leq r$.

Vamos agora definir duas novas aplicações, $f, g \in \mathcal{C}^{1}(\Omega \times] 0, T[)$, a valores reais, dadas por

$$
\begin{gathered}
f(x, t)=v\left(y_{r}, s_{r}\right)+\psi\left(x, y_{r}, t\right)+\frac{\left(t-s_{r}\right)^{2}}{\varepsilon} \\
g(y, s)=u\left(x_{r}, t_{r}\right)-\psi\left(x_{r}, y, t_{r}\right)-\frac{\left(t_{r}-s\right)^{2}}{\varepsilon} .
\end{gathered}
$$

É claro que ambas são continuamente diferenciáveis. Observe agora que $(u-f)\left(x_{r}, y_{r}, t_{r}, s_{r}\right)=$ $\theta\left(x_{r}, y_{r}, t_{r}, s_{r}\right)$. Assim, $\left(x_{r}, y_{r}, t_{r}, s_{r}\right)$ é ponto de máximo local de $u-f$ e, por ser $u$ uma sub-solução 
viscosa, devemos ter que:

$$
f_{t}\left(x_{r}, t_{r}\right)+H\left(x_{r}, t_{r}, u\left(x_{r}, t_{r}, D_{x} f\left(x_{r}, t_{r}\right) \leq 0 .\right.\right.
$$

Por outro lado, observamos que $v-g=-\theta$. Deste modo, $\left(x_{r}, y_{r}, t_{r}, s_{r}\right)$ é ponto de mínimo local de $v-g$ e, pelo fato de que $v$ é super-solução viscosa, deve valer que:

$$
g_{s}\left(y_{r}, s_{r}\right)+G\left(y_{r}, s_{r}, v\left(y_{r}, s_{r}, D_{x} g\left(y_{r}, s_{r}\right) \geq 0 .\right.\right.
$$

Ao fazermos as devidas substituições, chegamos a

$$
\psi\left(x_{r}, y_{r}, t_{r}\right)+\frac{2\left(t_{r}-s_{r}\right)}{\varepsilon}+H\left(x_{r}, t_{r}, u\left(x_{r}, t_{r}, D_{x} \psi\left(x_{r}, y_{r}, t_{r}\right) \leq 0 .\right.\right.
$$

e

$$
\frac{2\left(t_{r}-s_{r}\right)}{\varepsilon}+G\left(y_{r}, s_{r}, v\left(y_{r}, s_{r},-D_{y} \psi\left(x_{r}, y_{r}, t_{r}\right) \geq 0\right.\right.
$$

Multiplicando a segunda desigualdade por -1 e somando ambas, obtemos que

$$
\psi_{t}\left(x_{r}, y_{r}, t_{r}\right)+H\left(x_{r}, t_{r}, u\left(x_{r}, t_{r}\right), D_{x} \psi\left(x_{r}, y_{r}, t_{r}\right)\right)-G\left(y_{r}, s_{r}, v\left(y_{r}, s_{r}\right),-D_{y} \psi\left(x_{r}, y_{r}, t_{r}\right)\right) \leq 0 \text {. }
$$

Fazendo $r \longrightarrow 0$ obtemos que $x_{r} \longrightarrow a, y_{r} \longrightarrow b, t_{r} \longrightarrow c$ e $s_{r} \longrightarrow c$. Pela continuidade de $\psi_{t}$ e de $H$ e $G$, obtemos

$$
\psi_{t}(a, b, c)+H\left(a, c, u(a, c), D_{x} \psi(a, b, c)\right)-G\left(b, t, v(b, t),-D_{y} \psi(a, b, c)\right) \leq 0,
$$

demonstrando o resultado para o caso de um máximo estrito.

Suponha agora que $(a, b, c)$ seja apenas um máximo relativo para $(w-\psi)$. Então, construímos a função - que também é de classe $\mathcal{C}^{1}-\bar{\psi}$ dada por

$$
\bar{\psi}(x, y, t)=\psi(x, y, t)+\left\{\|x-a\|^{2}+\|y-b\|^{2}+\|t-c\|^{2}\right\}
$$

Então, claramente, $(w-\bar{\psi})$ tem um ponto de máximo estrito em $(a, b, c)$, e, portanto, vale que

$$
\bar{\psi}_{t}(a, b, c)+H\left(a, c, u(a, c), D_{x} \bar{\psi}(a, b, c)\right)-G\left(b, t, v(b, t),-D_{y} \bar{\psi}(a, b, c)\right) \leq 0 .
$$


Entretanto, como no ponto $(a, b, c)$, temos que $\bar{\psi} \equiv \psi$, o resultado segue.

Provaremos outro lema, que aborda a limitação superior de uma particular função, de grande importância na demonstração do teorema de comparação. Para tanto, discutiremos alguns resultados preliminares.

Proposição 14 Considere $\Omega \subset \mathbb{R}^{n}$, aberto e sejam $T>0$ e $\phi \in \mathcal{C}(\Omega \times \Omega \times[0, T])$. Então, se $0 \in \Omega$ e $s>0$ é tal que $\mathcal{B}_{s}(0) \subset \mathbb{R}^{2 n}$ está contida em $\Omega \times \Omega$, a função $\omega:[0, T] \longrightarrow \mathbb{R}$ definida por

$$
\omega(t)=\max \left\{\phi(x, y, t) \mid(x, y) \in \mathcal{B}_{\bar{s}}(0)\right\}
$$

é uniformemente contínua em $[0, T]$.

Demonstração. Fixemos $\varepsilon>0$ e observemos que $\phi$ é uniformemente contínua em $\overline{\mathcal{B}_{\bar{s}}(0)} \times[0, T]$, por ser este conjunto compacto e aquela função contínua. Assim sendo, existe $\delta(\varepsilon)>0$ com a propriedade de que

$$
\left(\left\|\left(x_{1}, y_{1}, t_{1}\right)-\left(x_{2}, y_{2}, t_{2}\right)\right\| \leq \delta(\varepsilon)\right) \Longrightarrow\left(\left|\phi\left(x_{1}, y_{1}, t_{1}\right)-\phi\left(x_{2}, y_{2}, t_{2}\right)\right| \leq \varepsilon\right)
$$

para todo $\left(x_{1}, y_{1}, t_{1}\right),\left(x_{2}, y_{2}, t_{2}\right) \in \mathcal{B}_{\bar{s}}(0) \times[0, T]$.

Tomemos agora $t, s \in[0, T]$ com $|t-s| \leq \delta(\varepsilon)$ e os pontos $\left(x_{t}, y_{t}\right)$ e $\left(x_{s}, y_{s}\right)$ tais que $\omega(t)=$ $\phi\left(x_{t}, y_{t}, t\right)$ e $\omega(s)=\phi\left(x_{s}, y_{s}, s\right)$. Claramente estes pontos existem, uma vez que a função é contínua e seu domínio é compacto. Se forem $\left(x_{1}, y_{1}\right) \in \mathcal{B}_{\delta}\left(\left(x_{t}, y_{t}\right)\right) \cup \mathcal{B}_{\bar{s}}(0)$ e $\left(x_{2}, y_{2}\right) \in \mathcal{B}_{\delta}\left(\left(x_{s}, y_{s}\right)\right) \cup \mathcal{B}_{\bar{s}}(0)$, concluímos que

$$
\omega(t)-\omega(s)>\varepsilon
$$

e

$$
\omega(s)-\omega(t)>\varepsilon
$$

o que implica no resultado.

Lema 11 Sejam $\Omega \subset \mathbb{R}^{n}$, com $\partial \Omega \neq \emptyset$ e $T>0$. Considere ainda $H: \Omega \times[0, T] \times \mathbb{R} \times \mathbb{R}^{n} \longrightarrow \mathbb{R}$ satisfazendo à condição 1 e sejam $u, v \in \mathcal{U C}_{m_{x}}(\bar{\Omega} \times[0, T])$ tais que

1. u é uma solução de viscosidade para

$$
u_{t}+H\left(x, t, u, D_{x} u\right) \leq 0
$$

e v é uma solução de viscosidade para

$$
v_{t}+H\left(x, t, v, D_{x} v\right) \geq 0
$$

2. $u \leq v e m \Omega \times\{0\} \cup \partial \Omega \times[0, T]$.

Então, segue que $u \leq v$ em $\Omega \times[0, T]$. 
Demonstração. Considere a função $d: \bar{\Omega} \longrightarrow \mathbb{R}$ dada por $d(x)=\operatorname{dist}(x, \partial \Omega)$. Por hipótese e pela proposição 13 segue que existe $r>0$ e $m \in \mathcal{M}$ tais que:

$$
|u(x, t)-u(y, t)|+|v(x, t)-v(y, t)| \leq m(\|x-y\|),
$$

para todo $(x, y, t) \in \bar{\Omega} \times \bar{\Omega} \times[0, T]$ tais que $\|x-y\| \leq r$ e

$$
u(x, t)-v(y, t) \leq m(\|x-y\|)+m(\min \{d(x), d(y)\})
$$

para todo $(x, y, t) \in \bar{\Omega} \times \bar{\Omega} \times[0, T]$ tais que $\|x-y\| \leq r$ e $\min \{d(x), d(y)\} \leq r$.

Definamos o conjunto $A=\{x \in \Omega \mid d(x) \geq r\}$. Claramente notamos que se $x \in \Omega \backslash A$, então, para todo $t \in[0, T]$,

$$
u(x, t)-v(x, t) \leq m(d(x)) \leq m(r) .
$$

Assim, mostraremos a limitação superior do conjunto $\mathcal{A}=\{u(a, t)-v(a, t) \mid(a, t) \in A \times[0, T]\}$. Tomemos $a \in A$,

$$
k=1+\frac{2 m(r)}{r^{2}}
$$

e definamos a aplicação $\Psi: \bar{\Omega} \times \bar{\Omega} \times[0, T] \longrightarrow \mathbb{R}$ por

$$
\Psi(x, y, t)=u(x, t)-v(y, t)-k\left(\|x-a\|^{2}+\|y-a\|^{2}\right) .
$$

Definiremos a função $\omega:[0, T] \longrightarrow \mathbb{R}$ por

$$
\omega(t)=\max \{\Psi(x, y, t) \mid\|x-a\| \leq r,\|y-a\| \leq r\} .
$$

De acordo com a proposição anterior, por ser $\Psi$ contínua e por ser $d(a) \geq r$, temos que $\omega$ é uniformemente contínua. Notemos que

$$
u(a, t)-v(a, t)=\Psi(a, a, t) \leq \omega(t) .
$$

Assim, precisamos mostrar que existe uma constante que não depende do particular $a \in A$ escolhido, digamos $K$, tal que $\omega \leq K$.

Vejamos que $\omega(0) \leq 2 m(r)$. Por ser contínua no compacto $\overline{\mathcal{B}_{r}(a)} \times \overline{\mathcal{B}_{r}(a)}$, a aplicação $\Psi(\cdot, \cdot, t)$ possui um ponto de máximo. Seja $\left(x_{0}, y_{0}\right)$ tal ponto. Então temos que $\omega(0)=\Psi\left(x_{0}, y_{0}, 0\right)$ e

$$
\begin{array}{r}
\Psi\left(x 0, y_{0}, 0\right)=u\left(x_{0}, 0\right)-v\left(y_{0}, 0\right)-k\left(\|x-a\|^{2}+\|y-a\|^{2}\right) \\
\leq u\left(x_{0}, 0\right)-u\left(y_{0}, 0\right) \leq\left(u\left(x_{0}, 0\right)-u(a, 0)\right)+\left(u(a, 0)-u\left(y_{0}, 0\right)\right) \\
\leq m\left(\left\|x_{0}-a\right\|\right)+m\left(\left\|y_{0}-a\right\|\right) \leq 2 m(r),
\end{array}
$$

e, com isto, $\mathcal{A}$ é limitado para $t=0$. Vejamos agora o que ocorre quando $\bar{t} \in(0, T]$. Suponha que $\omega(\bar{t}) \leq 0$. Então, $\omega(\bar{t}) \leq 2 m(r)$ e a limitação segue. Suponha agora que $\omega(\bar{t}) \geq 0$. 
Da continuidade de $\omega$ segue que $\omega^{-1}((0+\infty))$ é aberto em $[0, T)$. Denotaremos $\omega^{-1}(] 0+\infty[)$ por $\{\omega>0\}$. Segue ainda que existe $\alpha>0$ tal que

$$
\alpha=\inf \{t \in[0, \bar{t}] \mid[t, \bar{t}[\subset\{\omega>0\}\}
$$

Afirmamos que, se $\alpha \neq 0$, então $\omega(\alpha)=0$. Para vermos isto, basta notarmos que se fosse $\omega(\alpha) \neq 0$ então existiria uma vizinhança de $\omega(\alpha)$ e um ponto $z$ nesta vizinhança, tal que sua pré-imagem deveria ser menor do que $\alpha$, contrariando o fato de $\alpha$ ser ínfimo.

Seja $\beta \in(0, T]$ tal que $\bar{t} \in(\alpha, \beta]$ e $(\alpha, \beta) \subset\{\omega>0\}$. Utilizaremos aqui um fato que demonstraremos adiante. Trata-se da seguinte afirmação: $\exists C>0$, que não depende de $a$ tal que $\omega$ é solução viscosa de $\dot{\omega} \leq C$ em $\{\omega>0\}$.

Seja $\Phi:[\alpha, \beta] \longrightarrow[0, T]$ o difeomorfismo definido por

$$
\Phi(t)=\left(\frac{t-\alpha}{\beta-\alpha}\right) T
$$

Utilizando o fato anterior, e a proposição 14 , concluímos que $v=\omega \circ \Phi$ é uma solução de viscosidade de

$$
\dot{v} \frac{T}{\beta-\alpha} \leq C
$$

em $(0, T)$. Como este quociente é positivo, segue que $v$ é solução viscosa de

$$
\dot{v} \leq C \frac{\beta-\alpha}{T} .
$$

Então, do lema 10, segue que

$$
v(s) \leq v(0)+\int_{0}^{s} C \frac{\beta-\alpha}{T} d t
$$

para todo $s \in[0, T]$. Isto implica que

$$
\omega\left(\Psi^{-1}(s)\right) \leq \omega\left(\Psi^{-1}(0)\right)+C s \frac{\beta-\alpha}{T} .
$$

Como a pré-imagem por $\Psi$ de $s$ é algum $t \in[\alpha, \beta]$, a pré-imagem de $\alpha$ é zero, e $s(\beta-\alpha) \leq T^{2}$, segue que

$$
\omega(t) \leq \omega(\alpha)+C T,
$$

o que implica que, se $\alpha=0, \omega(t) \leq 2 m(r)+C T$ e se for $\alpha=0 \omega(t) \leq C T \leq C T+2 m(r)$. Assim, segue o resultado.

Devemos apenas demonstrar a afirmação que destacamos acima. Tomemos então um ponto $(x, y, t)$ em $\bar{\Omega} \times \bar{\Omega} \times[0, T]$ tais que suas duas primeiras coordenadas estejam na esfera de raio $r$ e centro $a$, i.e.,

$$
\|(x, y)-(a, a)\|=r .
$$


É fácil vermos que, das propriedades de módulo de continuidade, e do que fora discutido até este ponto,

$$
\begin{aligned}
& \Psi(x, y, t) \leq|u(x, t)-u(a, t)|+|v(y, t)-v(a, t)|+[u(a, t)-v(a, t)]-r^{2}-2 m(r) \\
& \quad \leq m(\|x-a\|)+m(\|y-a\|)+[u(a, t)-v(a, t)]-r^{2}-2 m(r) \\
& \quad \leq 2 m(r)+[u(a, t)-v(a, t)]-r^{2}-2 m(r) \\
& \quad<\Psi(a, a, t) .
\end{aligned}
$$

Afirmamos que existe $\left(x^{*}, y^{*}\right) \in\{(x, y) \mid\|x-a\|<r$ e $\|y-a\|<r\}=B$, dependendo de $t$ e $a \in \mathcal{A}$ tal que

$$
w(t)=u\left(x^{*}, t\right)-v\left(y^{*}, t\right)-k\left(\left\|x^{*}-a\right\|^{2}+\left\|y^{*}-a\right\|^{2}\right) .
$$

Isto é de fato verdade uma vez que $\Phi$ é contínua, o fecho de $B$ é compacto, e, mais ainda, para todo $t$ fixo, $\Phi<\Phi(a, a, t)$ em $\partial B$.

Considere agora a aplicação $\xi$ de classe $\mathcal{C}^{1}(\{w>0\})$ e tome um ponto $t_{0}$ em $\{w>0\}$ tal que

$$
(w-\xi)\left(t_{0}\right)-(w-\xi)(t) \geq 0
$$

em $\mathcal{B}_{\delta}\left(t_{0}\right)$, ou seja, $t_{0}$ é um máximo local para a diferença $w-\xi$. Podemos supor que

$$
w\left(t_{0}\right)=u\left(x^{*}, t_{0}\right)-v\left(y^{*, t_{0}}\right)-k\left(\left\|x^{*}-a\right\|^{2}+\left\|y^{*}-a\right\|^{2}\right) .
$$

Defina então as funções:

1. $\chi \in \mathcal{C}^{\infty}((0, T))$ com $\chi \equiv 1$ em $\left(t_{0}-\frac{\delta}{4}, t_{0}+\frac{\delta}{4}\right)$ e cujo suporte está contido em $\left(t_{0}-\frac{\delta}{2}, t_{0}+\frac{\delta}{2}\right)$.

2. $\zeta$ dada por

$$
\zeta(s) \doteq\left\{\begin{array}{cc}
\zeta(s) \chi(s) & s \in\left(t_{0}-\delta, t_{0}+\delta\right) \\
0 & s \in(0, T) \backslash\left(t_{0}-\delta, t_{0}+\delta\right) .
\end{array}\right.
$$

3. $\beta(x, y, t)=u(x, t)-v(y, t)$.

4. $\Xi(x, y, t)=k\left(\|x-a\|^{2}+\|y-a\|^{2}\right)+\zeta(t)$.

Por um lado, $\beta$ é uma solução viscosa de

$$
\beta_{t}+H\left(x, t, u, D_{x} \beta\right)-H\left(y, t, v,-D_{y} \beta\right) \leq 0,
$$

no conjunto $\Omega \times \Omega \times(0, T)$. Por outro lado, $\beta-\Xi$ tem um ponto de máximo local em $\left(x^{*}, y^{*}, t_{0}\right)$, uma vez que

$$
(\beta-\Xi)\left(x^{*}, y^{*}, t_{0}\right)=w\left(t_{0}\right)-\xi\left(t_{0}\right) \geq w(t)-\xi(t) \geq \Psi(x, y, t)-\zeta(t)=(\beta-\Xi)(x, y, t)
$$


Com isto,

$$
\Xi_{t}\left(x^{*}, y^{*}, t_{0}\right)+H\left(x^{*}, t_{0}, u\left(x^{*}, t_{0}\right), D_{x} \Xi\left(x^{*}, y^{*}, t_{0}\right)\right)-H\left(y^{*}, t_{0}, v\left(y^{*}, t_{0}\right),-D_{y} \Xi\left(x^{*}, y^{*}, t_{0}\right)\right) \leq 0,
$$

i.e.,

$$
\xi_{t}\left(t_{0}\right)+H\left(x^{*}, t_{0}, u\left(x^{*}, t_{0}\right), 2 k\left(x^{*}-a\right)\right)-H\left(y^{*}, t_{0}, v\left(y^{*}, t_{0}\right),-2 k\left(x^{*}-a\right)\right),
$$

onde $p=2 k\left(x^{*}-a\right)$ e $q=-2 k\left(y^{*}-a\right)$.

Da positividade de $w$ em $t_{0}$, temos $u\left(x^{*}, t_{0}\right)>v\left(y^{*}, t_{0}\right)$ e, mais ainda, pelas condições impostas sobre $H$,

$$
H\left(x^{*}, t_{0}, u\left(x^{*}, t_{0}\right), p\right)-H\left(y^{*}, t_{0}, v\left(y^{*}, t_{0}\right), q\right) \geq H\left(x^{*}, t_{0}, v\left(x^{*}, t_{0}\right), p\right)-H\left(y^{*}, t_{0}, v\left(y^{*}, t_{0}\right), q\right) .
$$

Sejam $M=2 k r$ e $m_{M}$ um módulo de continuidade satisfazendo o segundo item da condição 1 . O fato de $p, q \in \overline{\mathcal{B}_{M}(0)}$ e as propriedades usuais do módulo, implicam que

$$
H\left(x^{*}, t_{0}, v\left(x^{*}, t_{0}\right), p\right)-H\left(y^{*}, t_{0}, v\left(y^{*}, t_{0}\right), q\right) \geq-m_{M}(2 r(1-2 k)),
$$

o que implica, por sua vez, que

$$
H\left(x^{*}, t_{0}, u\left(x^{*}, t_{0}\right), p\right)-H\left(y^{*}, t_{0}, v\left(y^{*}, t_{0}\right), q\right) \geq-m_{M}(2 r(1-2 k)) .
$$

Assim, $\xi_{t}\left(t_{0}\right) \leq K$ onde $K=m_{M}(2 r(1+2 k))$ e, logo, $w$ é solução viscosa de

$$
w_{t} \leq K
$$

De posse desta série de resultados, demonstraremos agora o Teorema da Comparação.

Demonstração do Teorema da Comparação. Esta demonstração será feita por redução ao absurdo. Suponha que a afirmação do teorema seja falsa. Então, existe um ponto $\left(x^{*}, t^{*}\right) \in \Omega \times[0, T]$ tal que

$$
u\left(x^{*}, t^{*}\right)>v\left(x^{*}, t^{*}\right) .
$$

Da condição 1 podemos concluir que $t^{*}>0$ e, portanto, podemos definir a constante

$$
g=\frac{u\left(x^{*}, t^{*}\right)-v\left(x^{*}, t^{*}\right)}{2 t^{*}}>0 .
$$

Do lema anterior, existe um número estritamente positivo $\bar{K}$ com $u-v \leq \bar{K}$ em $\Omega \times[0, T]$. Multiplicando $g$ por $t$ obtém-se um número positivo, de onde vem que

$$
u(x, t)-v(x, t)-g t \leq \bar{K}
$$


em todo o domínio de $u$ e $v$. Usando a condição 1, item 4, obtemos que

$$
u(x, t)-v(x, t)-g t \leq 0
$$

para todo $(x, t) \in \partial \Omega \times[0, T]$. Juntando as desigualdades acima, obtemos que

$$
u(x, t)-v(x, t)-c t \leq \bar{K}
$$

em $\bar{\Omega} \times[0, T]$. Como $\left(x^{*}, t^{*}\right)$ é tal que a quantidade $u\left(x^{*}, t^{*}\right)-v\left(x^{*}, t^{*}\right)-c t^{*}$ é positiva, então o supremo do conjunto

$$
\{u(x, t)-v(x, t)-c t \mid x \in \bar{\Omega}, t \in[0, T]\}
$$

deve ser também positivo. Denotemos este supremo por $K$.

Sejam $\varepsilon, m$ e $m_{H}$ módulos de continuidade satisfazendo à mesma condição 1 . Então, da continuidade de ambos na origem, existe $u m \varepsilon_{0} \in(0, \min \{\varepsilon, 1\})$, tal que

$$
m_{H}\left(\varepsilon_{0}\right)<\frac{g}{4} \quad e \quad m\left(\varepsilon_{0}\right)<\min \left\{\frac{1}{2}, \frac{K}{2}\right\}
$$

e ainda existe um número $\varepsilon_{2}$ em $\left(0, \varepsilon_{0}\right)$ para o qual

$$
m\left(\varepsilon_{2}\right)<\frac{\varepsilon_{0}}{8}
$$

Definiremos agora uma função auxiliar que, como tem sido usual nestes argumentos, relaciona $u$ e $v$ e um termo cuja norma, em geral, conseguimos estimar. Sejam $\gamma$ um número real e

$$
\xi<\frac{\varepsilon_{2}^{2}}{16 m(1)}
$$

Então, defina $\bar{\Psi}: \bar{\Omega} \times \bar{\Omega} \times[0, T] \times(0,+\infty) \longrightarrow \mathbb{R}$ por

$$
\bar{\Psi}(x, y, t, \gamma)=u(x, t)-v(y, t)-c t-\left[\frac{\|x-y\|^{2}}{\xi}+\gamma\left(\|x\|^{2}+\|y\|^{2}\right)\right] .
$$

Nosso próximo passo será determinar um número real $\theta>0$ tal que $\Psi=\bar{\Psi}(\cdot, \cdot, \cdot, \theta)$ possua um ponto de máximo no conjunto $\Omega \times \Omega \times 0, T$.

Defina a função $\zeta:(0,+\infty) \longrightarrow \mathbb{R}$ por

$$
\zeta(\gamma)=K-\sup \{\bar{\Psi}(x, x, t, \gamma) \mid x \in \bar{\Omega} \text { e } t \in[0, T]\}
$$

É fácil ver que, na verdade, $\zeta$ não toma valores negativos. O que veremos é que, além disto,

$$
\lim _{\gamma \downarrow 0} \zeta(\gamma)=0
$$


Isto é equivalente a obtermos que

$$
\lim _{\gamma \downarrow 0} \sup \{\bar{\Psi}(x, x, t, \gamma) \mid x \in \bar{\Omega} \text { e } t \in[0, T]\}=K
$$

Da definição de supremo, afirmamos que existem $\hat{x}$ e $\hat{t}$ em $\bar{\Omega}$ e $[0, T]$ respectivamente, tais que, para qualquer $\mu>0$ tem-se:

$$
K-\frac{\mu}{2}<u(\hat{x}, \hat{t})-v(\hat{x}, \hat{y})-c \hat{t} \leq K
$$

Fixando $\mu$ positivo arbitrário e tomando $0<\gamma<\frac{\mu}{2\left(2\|\hat{x}\|^{2}+1\right)}$ temos que

$$
\begin{aligned}
K- & \mu<u(\hat{x}, \hat{t})-v(\hat{x}, \hat{t})-c \hat{t}-\frac{\mu}{2} \\
& <u(\hat{x}, \hat{t})-v(\hat{x}, \hat{t})-c \hat{t}-2 \gamma\|x\|^{2} \\
& <\sup \{\bar{\Psi}(x, x, t, \gamma) \mid x \in \bar{\Omega} \text { e } t \in[0, T]\} \\
& <K<K+\mu .
\end{aligned}
$$

Da arbitrariedade de $\mu$ e do fato de

$$
\mid \sup \{\bar{\Psi}(x, x, t, \gamma) \mid x \in \bar{\Omega} \text { e } t \in[0, T]\}-K \mid<\mu
$$

segue que

$$
\lim _{\gamma \downarrow 0} \zeta(\gamma)=0 .
$$

Da definição de $\delta(\gamma)$ e de $K$, temos que

$$
\sup \left\{\bar{\Psi}(x, y, t, \gamma) \mid(x, y, t) \in \bar{\Omega} \times \bar{\Omega} \times[0, T] \text { e }\|x-y\| \leq \varepsilon_{0}\right\} \geq K-\delta(\gamma)
$$

Tomemos $m_{R_{\xi}}$ onde

$$
R_{\xi}>2 \varepsilon_{0}+\frac{2 \varepsilon_{0}}{\xi}
$$

Por ser um módulo de continuidade, existe $\varepsilon_{3} \in\left(0, \varepsilon_{0}\right)$ tal que $m_{R_{\xi}}\left(\varepsilon_{3}\right)=\frac{g}{4}$. Assim, da construção de $\delta$ e do fato de que $\delta \longrightarrow 0$, existe um número $\zeta$ tal que

$$
0 \leq \delta(\zeta)<\min \left\{\frac{1}{2}, \frac{K}{2}, m(1), K-2 m\left(\varepsilon_{0}\right), \frac{\varepsilon_{0}}{8}\right\}
$$

Se denotarmos por $\Lambda$ o conjunto sobre o qual tomamos o supremo na equação 5.17 é fácil vermos que $\Lambda$ é fechado. Isto decorre da continuidade da norma e do fato elementar de que a interseção e união finita de conjuntos fechados é ainda um conjunto fechado. 
Pondo $s=\left(\frac{2}{\zeta}\right)^{\frac{1}{2}}$, podemos definir dois subconjuntos de $\Lambda$, a saber,

$$
\begin{gathered}
\Lambda_{1}=\Lambda \cap\left(\overline{\mathcal{B}_{s}(0)} \times \overline{\mathcal{B}_{s}(0)}\right) \times[0, T] \\
\Lambda_{2}=\Lambda \backslash \Lambda_{1}
\end{gathered}
$$

e $\Psi$ como definida anteriormente - dependendo de $\zeta$.

Das condições sobre $H$, da definição de $\Psi$ e das propriedades do módulo de continuidade, segue que

$$
\Psi(x, y, t) \leq K+m(\|x-y\|)-\frac{\|x-y\|^{2}}{\xi}-\zeta\left(\|x\|^{2}+\|y\|^{2}\right),
$$

Já da compacidade de $\Lambda_{1}$, juntamente com a continuidade de $\Psi$, sabemos que existe uma $3-$ upla $\left(x^{*}, y^{*}, t^{*}\right)$ em $\Lambda_{1}$ tal que

$$
\Psi(x, y, t) \leq \Psi\left(x^{*}, y^{*}, t^{*}\right) \quad \forall(x, y, t) \in \Lambda_{1}
$$

Nosso próximo passo será demonstrar que $\left(x^{*}, y^{*}, t^{*}\right)$ é um ponto de máximo global em $\Lambda$.

Por construção, $\delta(\zeta)<\frac{1}{2}$. Logo,

$$
\sup \{\Psi(x, y, t) \mid(x, y, t) \in \Lambda\} \geq K-\delta(\zeta)>K-\frac{1}{2} .
$$

Novamente, pela definição do supremo, podemos concluir que existe um ponto $\left(x_{0}, y_{0}, t_{0}\right) \in \Lambda$ tal que $K-\frac{1}{2}<\Psi\left(x_{0}, y_{0}, t_{0}\right)$.

Tomemos $(x, y, t)$ em $\Lambda_{2}$ e suponhamos que seja $\|x\|>s$. Segue:

$$
\begin{aligned}
& \Psi(x, y, t) \leq K+m(\|x-y\|)-\zeta\|x\|^{2} \\
& \quad \leq K+m\left(\varepsilon_{0}\right)-\zeta s^{2} \\
& \quad<\Psi\left(x_{0}, y_{0}, t_{0}\right)+\frac{1}{2}+m\left(\varepsilon_{0}\right)-\zeta s^{2} \\
& \quad<\Psi\left(x_{0}, y_{0}, t_{0}\right)-1<\Psi\left(x_{0}, y_{0}, t_{0}\right) .
\end{aligned}
$$

Logo, $\left(x_{0}, y_{0}, t_{0}\right)$ é um ponto de máximo para $\Psi$ em $\Lambda_{2}$. Facilmente concluímos que $\left(x^{*}, y^{*}, t^{*}\right)$ é um ponto de máximo global para $\Psi$ em $\Lambda$.

Mostraremos agora que este ponto pertence na verdade ao interior de $\Lambda$, ou seja, queremos mostrar que

$$
\left(x^{*}, y^{*}, t^{*}\right) \in \Omega \times \Omega \times(0, T) .
$$

Das definições de $K, \delta$ e $\zeta$ obtemos que

$$
K-\delta(\zeta) \leq \Psi\left(x^{*}, y^{*}, t^{*}\right) \leq K+m\left(\left\|x^{*}-y^{*}\right\|\right)-\left[\frac{\left\|x^{*}-y^{*}\right\|^{2}}{\xi}+\zeta\left(\left\|x^{*}\right\|^{2}+\left\|y^{*}\right\|^{2}\right)\right]
$$


$\mathrm{e}$

$$
\frac{\left\|x^{*}-y^{*}\right\|^{2}}{\xi} \leq \frac{\left\|x^{*}-y^{*}\right\|^{2}}{\xi}+\zeta\left(\|x\|^{2}+\|y\|^{2}\right) \leq \delta(\zeta)+m\left(\left\|x^{*}-y^{*}\right\|\right) .
$$

As majorações de $\delta(\zeta)$, nos garantem que

$$
\delta(\zeta) \leq\left\{\begin{array}{c}
m(1) \\
K-2 m\left(\varepsilon_{0}\right)
\end{array}\right.
$$

e, portanto,

$$
\left\|x^{*}-y^{*}\right\| \leq 2 \sqrt{m(1)}
$$

e

$$
\frac{\left\|x^{*}-y^{*}\right\|^{2}}{\xi} \leq \delta(\zeta)+m(2 \sqrt{m(1) \xi}) .
$$

Seja $d$ a função definida na proposição 13 . Então é imediato que se $d\left(x^{*}\right)$ ou $d\left(y^{*}\right)$ forem menores do que $\varepsilon_{0}$, da arbitrariedade deste, segue que $x^{*}$ ou $y^{*}$ são pontos de $\partial \Omega$. Suponhamos então que $d\left(x^{*}\right)<\varepsilon_{0}$. Temos então que

$$
\begin{aligned}
K- & \delta(\zeta) \leq \Psi\left(x^{*}, y^{*}, t^{*}\right) \leq u\left(x^{*}, t^{*}\right)-v\left(y^{*}, t^{*}\right) \\
& \leq m\left(\left\|x^{*}-y^{*}\right\|\right)+m\left(\min \left\{d\left(x^{*}\right), d\left(y^{*}\right)\right\}\right) \\
& \leq 2 m\left(\varepsilon_{0}\right)<K-\delta(\zeta),
\end{aligned}
$$

o que é um absurdo. Logo $\left(x^{*}, y^{*}\right) \in \Omega \times \Omega$. Resta-nos mostrar agora que $t^{*} \in(0, T)$. Para tanto, supomos inicialmente que $t^{*}=0$ e em seguida supomos que $t^{*}=T$. Em ambos os casos, obteremos contradições, o que implicará imediatamente na inclusão que devemos estabelecer.

Seja $t^{*}=0$. Então se obtém a desigualdade

$$
\begin{aligned}
K- & \delta(\zeta) \leq \Psi\left(x^{*}, y^{*}, 0\right) \leq u\left(x^{*}, 0\right)-v\left(y^{*}, 0\right) \\
& \leq v\left(x^{*}, 0\right)-v\left(y^{*}, 0\right) \leq m\left(\left\|x^{*}-y^{*}\right\|\right) \leq m\left(\varepsilon_{0}\right)<\frac{K-\delta(\zeta)}{2}<K-\delta(\zeta),
\end{aligned}
$$

o que é um absurdo, e, de forma análoga ao que foi feito para o par $\left(x^{*}, y^{*}\right)$, vemos que $t^{*}>0$.

Neste instante vamos recorrer a duas funções que já soam familiares aos argumentos deste capítulo. São elas

$$
w(x, y, t)=u(x, t)-v(y, t)
$$

e

$$
\Phi(x, y, t)=g t+\frac{\|x-y\|^{2}}{\xi}+\zeta\left(\|x\|^{2}+\|y\|^{2}\right)
$$


além de

$$
\begin{gathered}
p=\frac{2\left(x^{*}-y^{*}\right)}{\xi}, \\
q_{x}=2 \zeta x^{*}
\end{gathered}
$$

$\mathrm{e}$

$$
q_{y}=2 \zeta y^{*}
$$

É evidente que $\left\|q_{i}\right\| \leq 2 \zeta s$ para $i=x, y$, donde obtém-se que

$$
\max \left\{\left\|q_{i}\right\| \mid i=x, y\right\}<\varepsilon_{3} .
$$

Sabemos que $w$ e uma solução viscosa de

$$
w_{t}+H\left(x, t, u, D_{x} w\right)-H\left(y, t, v,-D_{y} w\right) \leq 0
$$

no aberto $\Omega \times \Omega \times(0, T)$. Por outro lado, é fácil ver que $\left(x^{*}, y^{*}, t^{*}\right)$ é um ponto de máximo de $w-\Phi$.

Assim, a proposição anterior, com $t^{*}=T$ nos fornece

$$
g+H\left(x^{*}, t^{*}, u\left(x^{*}, t^{*}\right), p+q_{x}\right)+H\left(y^{*}, t^{*}, v\left(y^{*}, t^{*}\right), p+q_{y}\right) \leq 0 .
$$

Utilizando as condições impostas sobre $H$, provaremos a negatividade de $g$. Com isto, concluímos a demonstração do Teorema da Comparação.

É claro que $\Psi\left(x^{*}, y^{*}, t^{*}\right)>0$. Então $u\left(x^{*}, t^{*}\right)>v\left(y^{*}, t^{*}\right)$. Da condição de monotonicidade de $H$ na terceira variável segue que

$$
H\left(x^{*}, t^{*}, u\left(x^{*}, t^{*}\right), p+q_{x}\right)>H\left(x^{*}, t^{*}, v\left(y^{*}, t^{*}\right), p+q_{x}\right) .
$$

Ainda pelas condições sobre $H$, temos que

$$
H(\tilde{x}, \tilde{t}, \tilde{r}, \lambda(\tilde{x}-\tilde{y}))-H(\tilde{y}, \tilde{t}, \tilde{r}, \lambda(\tilde{x}-\tilde{y})) \geq-m_{H}\left(\lambda\|\tilde{x}-\tilde{y}\|^{2}+\|\tilde{x}-\tilde{y}\|\right),
$$

para todo $(\tilde{x}, \tilde{y}, \tilde{t}, \tilde{r}, \lambda) \in \mathbb{R}^{n} \times \mathbb{R}^{n} \times[0, T] \times \mathbb{R} \times \mathbb{R}_{+}$.

Fixando

$$
(\tilde{x}, \tilde{y}, \tilde{t}, \tilde{r}, \lambda)=\left(x^{*}, y^{*}, t^{*}, v\left(y^{*}, t^{*}\right), \frac{2}{\xi}\right),
$$

a desigualdade acima torna-se

$$
H\left(x^{*}, t^{*}, v\left(y^{*}, t^{*}\right), p\right)-H\left(y^{*}, t^{*}, v\left(y^{*}, t^{*}\right), p\right) \geq-m_{H}\left(\frac{2}{\xi}\left\|x^{*}-y^{*}\right\|^{2}+\left\|x^{*}-y^{*}\right\|\right) .
$$


Uma vez que nossas estimativas anteriores nos garantem que

$$
\frac{2}{\xi}\left\|x^{*}-y^{*}\right\|+\left\|x^{*}-y^{*}\right\|<\varepsilon_{0}
$$

é claro que

$$
m_{H}\left(\frac{2}{\xi}\left\|x^{*}-y^{*}\right\|+\left\|x^{*}-y^{*}\right\|\right) \leq m_{H}\left(\varepsilon_{0}\right)<\frac{g}{4} .
$$

Neste ponto, podemos escrever

$$
\begin{array}{r}
-g \geq H\left(x^{*}, t^{*}, u\left(x^{*}, t^{*}\right), p+q_{x}\right)-H\left(y^{*}, t^{*}, v\left(y^{*}, t^{*}\right), p+q_{y}\right) \\
\quad \geq H\left(x^{*}, t^{*}, v\left(y^{*}, t^{*}\right), p+q_{x}\right)-H\left(y^{*}, t^{*}, v\left(y^{*}, t^{*}\right), p+q_{y}\right)
\end{array}
$$

Somando e subtraindo na desigualdade acima a expressão

$$
H\left(x^{*}, t^{*}, v\left(y^{*}, t^{*}\right), p\right)-H\left(y^{*}, t^{*}, v\left(y^{*}, t^{*}\right), p\right)
$$

obtemos que

$$
\begin{aligned}
-g & \geq-m_{R_{\xi}}\left(\left\|q_{x}\right\|\right)-m_{H}\left(\frac{2}{\xi}\left\|x^{*}-y^{*}\right\|^{2}+\left\|x^{*}-y^{*}\right\|\right) \\
& \geq-2 m_{R_{\xi}}\left(\varepsilon_{3}\right)-m_{H}\left(\varepsilon_{0}\right) \\
& \geq-2 \frac{g}{4}-\frac{g}{4}=-\frac{3 g}{4},
\end{aligned}
$$

o que implica que $g$ é menor ou igual a zero e nos leva a um absurdo.

Assim, o teorema de unicidade de soluções viscosas para o problema dado por 5.14 e 5.15 está demonstrado. Desta maneira, concluímos que este possuirá no máximo uma solução. O conteúdo da próxima seção é dedicado à demonstração de que de fato existirá uma solução e, portanto, nossa conclusão será que, dado um particular problema de Cauchy, existirá uma única solução viscosa.

\subsubsection{Um teorema de existência}

Nesta subseção, consideramos o problema de dados iniciais

$$
u_{t}+H(x, u(x, t), D u(x, t))=0
$$

em $\Omega \times[0, T]$, com as condições iniciais

$$
u \equiv \phi
$$


onde $\Omega \subset \mathbb{R}^{n}$ é um aberto e $\phi$ é uma função, a princípio, qualquer.

Antes da exposição do teorema de existência de soluções viscosas, vamos discutir um lema auxiliar.

Lema 12 Sejam $\Omega \subset \mathbb{R}$ um aberto, $F \in \mathcal{C}\left(\Omega \times \mathbb{R} \times \mathbb{R}^{n}\right), u \in \mathcal{C}(\Omega)$ e as seqüências $\left(F_{n}\right)_{n \in \mathbb{N}} e$ $\left(u_{n}\right)_{n \in \mathbb{N}}$ em, respectivamente, $\mathcal{C}\left(\Omega \times \mathbb{R} \times \mathbb{R}^{n}\right)$ e $\mathcal{C}(\Omega)$, tais que as seguintes condições são satisfeitas:

1. $\left(F_{n}\right)_{n \in \mathbb{N}}$ converge uniformemente para $F$ sobre compactos de $\Omega \times \mathbb{R} \times \mathbb{R}^{n}$,

2. $\left(u_{n}\right)_{n \in \mathbb{N}}$ converge uniformemente para u sobre compactos de $\Omega$

3.

$$
\frac{-1}{n} \Delta u_{n}+F_{n}\left(x, u_{n}, D u_{n}\right)=0
$$

em $\Omega$ para todo $n \in \mathbb{N}$.

Então, dados $\zeta \in \mathcal{C}^{2}(\Omega)$ e $z_{0}$ um ponto de máximo local de $u-\zeta$,

$$
F\left(z_{0}, u\left(z_{0}\right), D \zeta\left(z_{0}\right)\right) \leq 0
$$

Demonstração. Tomemos a função $f$ de classe $\mathcal{C}^{\infty}$ sobre $\Omega$ tal que

$$
f(y) \doteq\left\{\begin{array}{cc}
1 & \text { se } y=y_{0} \\
s \in[0,1) & \text { c.c. }
\end{array}\right.
$$

Claramente, $y_{0}$ é um ponto de máximo local estrito de $(u-(\zeta-f))$. Seja $K$ o fecho de alguma vizinhança de $y_{0}$ em que este é um máximo local de $(u-(\zeta-f))$.

Como $\left(u_{n}-(\zeta-f)\right) \stackrel{u}{\longrightarrow}(u-(\zeta-f))$ podemos obter uma subseqüência $\left(u_{n_{k}}\right)_{k \in \mathbb{N}}$ e uma seqüência $y_{k}$ tal que $y_{k} \longrightarrow y_{0}$ e $y_{k}$ é um ponto de máximo de $u_{n_{k}}-(\zeta-f)$ para todo $k \in \mathbb{N}$. Segue então que $\forall k \in \mathbb{N}$,

$$
F_{n_{k}}\left(y_{k}, u_{n_{k}}\left(y_{k}\right), D(\zeta-f)\left(y_{k}\right)\right)=F_{n_{k}}\left(y_{k}, u_{n_{k}}\left(y_{k}\right) D u_{n_{k}}\left(y_{k}\right)\right)=\frac{\Delta u_{n_{k}}\left(y_{k}\right)}{n_{k}} \leq \frac{\Delta(\zeta-f)\left(y_{k}\right)}{n_{k}},
$$

o que implica que

$$
F\left(y_{0}, u\left(y_{0}\right), D \zeta\left(y_{0}\right)\right)=F\left(y_{0}, u\left(y_{0}\right), D(\zeta-f)\left(y_{0}\right)\right) \leq 0
$$

levando exatamente ao resultado do lema.

Teorema 13 (Existência de solução viscosa) Sejam $\Omega \subset \mathbb{R}$ um aberto, $F \in \mathcal{C}\left(\Omega \times \mathbb{R} \times \mathbb{R}^{n}\right)$, $u \in \mathcal{C}(\Omega)$ e as seqüências $\left(F_{n}\right)_{n \in \mathbb{N}}$ e $\left(u_{n}\right)_{n \in \mathbb{N}}$ em, respectivamente, $\mathcal{C}\left(\Omega \times \mathbb{R} \times \mathbb{R}^{n}\right)$ e $\mathcal{C}(\Omega)$, tais que as seguintes condições são satisfeitas:

1. $\left(F_{n}\right)_{n \in \mathbb{N}}$ converge uniformemente para $F$ sobre compactos de $\Omega \times \mathbb{R} \times \mathbb{R}^{n}$, 
2. $\left(u_{n}\right)_{n \in \mathbb{N}}$ converge uniformemente para u sobre compactos de $\Omega$

3.

$$
\frac{-1}{n} \Delta u_{n}+F_{n}\left(x, u_{n}, D u_{n}\right)=0
$$

em $\Omega$ para todo $n \in \mathbb{N}$.

Então u é solução de viscosidade de $F(x, u, D u)=0$ em $\Omega$.

Uma vez demonstrado o teorema acima, se fizermos

$$
F(x, u, D u)=u_{t}+H(x, u, D u)
$$

então está garantida a existência de solução viscosa do problema de nosso interesse.

Demonstração do Teorema de Existência. Começamos nossa demonstração provando que $u$ é sub-solução viscosa de $F=0$. Como é usual nesta argumentação, considere uma função $\Psi$ continuamente diferenciável definida sobre o domínio $\Omega$ e tome um ponto $x_{0} \in \Omega$ que é um ponto de máximo de $u-\Psi$.

Tomemos um função $\xi$ que separa $\Omega$ e $x^{*}$ de modo que

$$
\xi(x) \doteq\left\{\begin{array}{cc}
1 & \text { se } x=x_{0} \\
s \in[0,1) & \text { c.c. }
\end{array}\right.
$$

$\operatorname{com} \xi$ de classe $\mathcal{C}^{\infty}$ sobre $\Omega$. Claramente existe $\varepsilon>0$ tal que

$$
(u-(\Psi-\xi))(x)<(u-(\Psi-\xi))\left(x_{0}\right)
$$

para todo $x \in \mathcal{B}_{\varepsilon}\left(x_{0}\right)$.

Defina agora as funções $\Xi$ sobre $\mathbb{R}^{n}$ tal que $\Xi \equiv 1$ em $\mathcal{B}_{\frac{\varepsilon}{4}}\left(x_{0}\right)$ com suporte contido em $\mathcal{B}_{\frac{\varepsilon}{2}}\left(x_{0}\right)$ e $\zeta$ sobre $\mathbb{R}^{n}$ a função dada por

$$
\zeta(x) \doteq\left\{\begin{array}{cc}
\Xi(x) \Psi(x) & \text { se } x \in \mathcal{B}_{\varepsilon}\left(x_{0}\right) \\
0 & \text { c.c. }
\end{array}\right.
$$

Então, se assumirmos que $\Xi$ é de classe $\mathcal{C}^{\infty}$ segue que $\zeta$ é de classe $\mathcal{C}^{1}\left(\mathbb{R}^{n}\right)$ e

$$
\begin{gathered}
D \zeta\left(x_{0}\right)=D \Psi\left(x_{0}\right), \\
\left(u-\left(\zeta_{\mid \Omega}-\xi\right)\right)\left(x_{0}\right) \geq\left(u-\left(\zeta_{\mid \Omega}-\xi\right)\right)(x)
\end{gathered}
$$

para todo $x$ em alguma vizinhança de $x_{0}$.

Tomemos então uma seqüência de funções de classe $\mathcal{C}^{2}$ sobre $\mathbb{R}^{n}$, nominalmente, $\zeta_{n}$, tal que

$$
\zeta_{n} \stackrel{u}{\longrightarrow} \zeta
$$


e

$$
D \zeta_{n} \stackrel{u}{\longrightarrow} D \zeta .
$$

Então,

$$
\left(u-\left(\zeta_{n \mid \Omega}-\xi\right)\right)_{n \in \mathbb{N}} \stackrel{u}{\longrightarrow}\left(u-\left(\zeta_{\mid \Omega}-\xi\right)\right)
$$

em $\overline{\mathcal{B}\left(x_{0}\right)}$

Tomemos uma subseqüência $\left(\zeta_{n_{k}}\right)$ e uma seqüência $x_{k}$ de pontos de $\mathbb{R}^{n}$ com $x_{k} \longrightarrow x_{0}$ e tal que

$$
\left(u-\left(\zeta_{n_{k}}-\xi\right)\right)\left(x_{k}\right) \geq\left(u-\left(\zeta_{n_{k}}-\xi\right)\right)(x)
$$

para todo $x$ numa vizinhança de $x_{k}$, para todo $k \in \mathbb{N}$.

Defina $\pi_{k}=\zeta_{n_{k \mid \Omega}}-\xi$. É claro que $\pi_{k} \in \mathcal{C}^{2}(\Omega)$ e, para todo $k \in \mathbb{N}, x_{k}$ é um ponto de máximo local de $u-\pi_{k}$. Do lema anterior temos que

$$
F\left(x_{k}, u\left(x_{k}\right), D\left(\xi_{n_{k}}-\xi\right)\left(x_{k}\right)\right)=F\left(x_{k}, u\left(x_{k}\right), D \pi_{k}\left(x_{k}\right)\right) \leq 0 .
$$

Tomando os limites uniformes, da continuidade de $F$, segue que

$$
F(x, u(x), D \Psi(x)) \leq 0
$$

e então, $u$ é sub-solução viscosa de $F=0$.

Para verificarmos que $u$ é super-solução viscosa e, portanto, solução viscosa de $F=0$, o argumento é análogo.

Dois elementos importantes devem ser mencionados nesta altura. O primeiro deles refere-se à existência de solução para o problema

$$
\frac{-1}{n} \Delta u_{n}+F_{n}\left(x, u_{n}, D u_{n}\right)=0
$$

De fato, verificar a existência de solução neste caso é um exercício bastante elaborado e não uma tarefa trivial, como o enunciado do resultado pode sugerir. Para uma discussão deste aspecto do teorema, referimo-nos a [10].

O segundo comentário refere-se ao chamado método de Perron. No que diz respeito à existência de soluções viscosas para a equação de Hamilton-Jacobi, o método de Perron é uma ferramenta poderosa introduzida neste contexto por Ishii [33]. Para uma discussão detalhada sugerimos a leitura de [12].

Agora, todos os elementos concernentes à teoria das soluções viscosas da equação de HJ que são necessários à abordagem dos jogos diferenciais estão delineados. Passamos então, no próximo capítulo, à aplicação destas noções e, assim, ao estabelecimento da existência da função de valor de Friedman. 


\section{Capítulo 6}

\section{Revisitando a Teoria dos Jogos Diferenciais}

Neste capítulo retomaremos a noção de jogo diferencial como formulada por Friedman - e exposta no capítulo 3 - com o objetivo de estudar alguns aspectos relacionados com a teoria das soluções viscosas da equação HJI.

A princípio, apresentaremos a definição do jogo diferencial e das estratégias não-antecipativas como formuladas por Elliot e Kalton [14] e, mais recentemente, por Bardi e Capuzzo-Dolcetta [10]. Em seguida, daremos uma demonstração do princípio da programação dinâmica para a equação de HJI e apresentaremos uma propriedade de continuidade da função de valor, a saber, mostraremos que a função de valor é Hölder contínua. Então, mostraremos que as funções de valor - superior e inferior - de um jogo diferencial são soluções viscosas da HJI e, mais ainda, através deste resultado e do que foi discutido no capítulo anterior, derivaremos propriedades da função de valor do jogo e as condições sob as quais um jogo possui uma função de valor segundo Friedman.

\subsection{Introdução}

Nesta seção vamos introduzir o chamado jogo diferencial segundo Friedman, de acordo com suas apresentações mais recentes na literatura.

Novamente tratamos jogos de duas pessoas, de soma zero e consideramos a seguinte dinâmica

$$
\dot{x}(t)=f(x(t), u(t), v(t)), \quad t>0 \quad e \quad x(0)=x_{0},
$$

onde $f: \mathbb{R}^{n} \times U \times V \longrightarrow \mathbb{R}^{n}$, com $U$ e $V$ espaços métricos compactos, sendo que $f$ ainda satisfaz uma condição de Lipschitz dada pela expressão

$$
(f(x, a, b)-f(y, a, b)) \cdot(x-y) \leq L\|x-y\|^{2},
$$

com $L$ uma constante positiva.

As funções $u$ e $v$ são, respectivamente, os controles dos jogadores 1 e 2 e, formalmente, são elementos dos conjuntos

$$
\mathcal{U}=\{u:[0, \infty[\longrightarrow U \mid u \text { é mensurável }\}
$$

e

$$
\mathcal{V}=\{v:[0, \infty[\longrightarrow V \mid v \text { é mensurável }\}
$$


A função $x_{x_{0}}$ denotará a solução de $6.1 \mathrm{e}$, devido à condição 6.2 , valem as seguintes estimativas: ${ }^{1}$

$$
\begin{array}{r}
\left\|x_{x_{0}}(t, u(t), v(t))-x_{y_{0}}(t, u(t), v(t))\right\| \leq e^{L t}\left\|x_{0}-y_{0}\right\|, \quad t>0, \\
\left\|x_{x_{0}}(t, u(t), v(t))-x_{0}\right\| \leq M_{x_{0}} t, \quad t \in\left[0, \frac{1}{M_{x_{0}}}\right], \\
\left\|x_{x_{0}}(t, u(t), v(t))\right\| \leq(\|x\|+\sqrt{2 K t}) e^{K t},
\end{array}
$$

para todo $u \in \mathcal{U}$ e todo $v \in \mathcal{V}$, sendo as constantes $M_{x_{0}}$ e $K$ dadas por

$$
M_{x_{0}}=\max \left\{\|f(y, u(t), v(t))\| \mid\left\|x_{0}-y\right\| \leq 1, u \in U, v \in V\right\}
$$

$\mathrm{e}$

$$
K=L+\max \{\|f(0, u(t), v(t))\| \mid u \in U, v \in V\} .
$$

Associada a esta dinâmica existe uma aplicação $\mathcal{P}\left(x_{0}, u(t), v(t)\right)$, a função de pagamento do jogo, que o jogador 1 tentará maximizar e o jogador 2 tentará minimizar.

O jogo diferencial segundo Friedman possui um padrão de informação diferente daquele descrito por Isaacs. Aqui, os jogadores decidem no instante $t$ sem conhecer as ações de seu oponente em instantes futuros. Mais do que isso, as noções de jogo superior e jogo inferior sugerem que, em cada um destes, um jogador decidirá seu controle no instante $t$ conhecendo as escolhas de seu oponente até o mesmo instante $t$, sendo que o outro apenas conhecerá as ações do oponente até o instante imediatamente anterior. Vejamos isto com mais clareza através da definição de estratégias não-antecipativas.

Definição 48 Uma estratégia para o jogador 1 é uma aplicação $\mu: \mathcal{V} \longrightarrow \mathcal{U}$. Ela será dita nãoantecipativa se, para qualquer $t>0$ e quaisquer dois elementos $v, \bar{v} \in \mathcal{V}$ tais que $v(s)=\bar{v}(s)$ para todo $s \leq t$ tivermos que $\mu[v](s)=\mu[\bar{v}](s)$ para todo $s \leq t$. O conjunto das estratégias nãoantecipativas do jogador 1 será denotado $\Gamma$. Formalmente:

$$
\Gamma=\{\mu: \mathcal{V} \longrightarrow \mathcal{U} \mid v(s)=\bar{v}(s) \forall s \leq t \Longrightarrow \mu[v](s)=\mu[\bar{v}](s) \forall s \leq t\} .
$$

Por outro lado, uma estratégia para o jogador 2 é uma aplicação $\nu: \mathcal{U} \longrightarrow \mathcal{V}$. Analogamente, ela é dita não-antecipativa se, para qualquer $t>0$ e quaisquer dois $u, \bar{u} \in \mathcal{U}$ tais que $u(s)=\bar{u}(s)$ para todo $s \leq t$ tivermos que $\nu[u](s)=\nu[\bar{u}](s)$. Chamaremos o conjuntos das estratégias nãoantecipativas do jogador 2 de $\Delta$, e, novamente, temos:

$$
\Delta=\{\nu: \mathcal{U} \longrightarrow \mathcal{V} \mid u(s)=\bar{u}(s) \forall s \leq t \Longrightarrow \nu[u](s)=\nu[\bar{u}](s) \forall s \leq t\}
$$

Definição 49 Considere um jogo diferencial como usualmente descrito com função de pagamento

\footnotetext{
$[30]$.

${ }^{1}$ Para estas estimativas, remetemos o leitor ao clássico texto em equações diferencias ordinárias de Philip Hartman
} 
dada por $\mathcal{P}: \mathbb{R}^{n} \times \mathcal{U} \times \mathcal{V} \longrightarrow \mathbb{R}$. Então, o valor inferior do jogo é dado por

$$
\vartheta_{b}\left(x_{0}\right)=\inf _{\mu \in \Gamma} \sup _{v \in \mathcal{V}} \mathcal{P}\left(x_{0}, \mu[v], v\right)
$$

Já o valor superior do jogo é dado por

$$
\vartheta_{\sharp}\left(x_{0}\right)=\sup _{\nu \in \Delta} \inf _{u \in \mathcal{U}} \mathcal{P}\left(x_{0}, u, \nu[u]\right) .
$$

A noção de estratégia não-antecipativa introduz uma idéia bastante importante do ponto de vista do jogo diferencial. No jogo inferior, o jogador 1 toma suas decisões no instante $t^{*}$ conhecendo todas as decisões do jogador 2 até o instante $t^{*}$ inclusive, ao passo que este toma decisões conhecendo apenas as escolhas do jogador 1 até o instante $t<t^{*}$. Deste modo, o jogador 1 possui uma vantagem sobre o jogador 2 , na medida em que possui mais informação no momento de sua decisão. O mesmo se verifica na direção oposta - em favor do jogador 2 - no caso do jogo superior.

Novamente, nosso interesse é que o jogo tenha uma função valor, ou seja, que $\vartheta_{b}\left(x_{0}\right)=\vartheta_{\sharp}\left(x_{0}\right)$. Usaremos a teoria das soluções viscosas da equação de HJI para obtermos as condições sob as quais isto se verifica.

Antes disto, porém, vejamos alguns exemplos de estratégias não-antecipativas e um exemplo de uma estratégia que não é não-antecipativa.

\section{Exemplo 10}

1 Estratégia constante - considere a estratégia $\mu \equiv \bar{u} \in \mathcal{U}$ tal que $\mu[v] \equiv \bar{u}$ para todo $v \in \mathcal{V}$. Esta é claramente uma estratégia não-antecipativa.

2 Seja $\Psi: V \longrightarrow U$ tal que $\Psi(v(\cdot))$ é mensurável para todo $v \in \mathcal{V}$. Então a estratégia $\mu[v](s)=$ $\Psi(v(s))$ é não-antecipativa.

3 Seja $\mu$ uma distribuição de probabilidade sobre o intervalo $[0, t]$ e considere o funcional

$$
I(t)=\int_{0}^{t} x(s) \mu
$$

Então, a aplicação $\Phi(I(t), t)$, que gera a estratégia $\mu[v](t)=\Phi(I(t), t)$ é uma estratégia nãoantecipativa, na medida em que não depende de $v$ mas sim de uma média ponderada da história passada dos estados do jogo até o instante $t$.

4 Vejamos agora um exemplo de uma estratégia que não é não-antecipativa. Seja $\tau>0$ fixo e considere a aplicação

$$
\mu[v](s)=\Psi(v(s+\tau))
$$

onde $\Psi(v(\cdot+\tau))$ é mensurável. Considere então as aplicações $v(s)$ e $\bar{v}(s)$ dadas por

$$
v(s)=s^{2} \quad \text { se } s \in\left[0, \tau\left[\quad \text { e } \quad v(s)=\tau^{2} \quad \text { se } s \geq \tau\right.\right.
$$




$$
\bar{v}(s)=s^{2} \quad \text { se } s \in\left[0, \tau\left[\quad \text { e } \quad \bar{v}(s)=\tau^{2}-(\tau-s) s \quad \text { se } s \geq \tau .\right.\right.
$$

Então, claramente, para qualquer $s \in[0, \tau[$ temos que $v(s)=\bar{v}(s)$, entretanto, $v(s+\tau) \neq$ $\bar{v}(s+\tau)$, o que implica que $\Psi(v(s+\tau)) \neq \Psi(\bar{v}(s+\tau))$.

Em comparação com o jogo em que ocorrem estratégias não-antecipativas, considere o jogo chamado estático, em que os jogadores apenas tomam suas escolhas sem informação sobre as ações de seus oponentes. Então analogamente teremos as funções de valor inferior e superior estáticas dadas por

$$
\vartheta_{b}^{s}\left(x_{0}\right)=\min _{u \in \mathcal{U}} \max _{v \in \mathcal{V}} \mathcal{P}\left(x_{0}, u, v\right)
$$

$\mathrm{e}$

$$
\vartheta_{\sharp}^{s}\left(x_{0}\right)=\max _{v \in \mathcal{V}} \min _{u \in \mathcal{U}} \mathcal{P}\left(x_{0}, u, v\right) .
$$

Estes seriam os valores inferiores e valores superiores segundo Isaacs e, de acordo com o que vimos no segundo capítulo, sabemos quais as condições sobre $\mathcal{P}$ para que ambos sejam iguais. Entretanto, é possível obtermos algumas relações entre estes valores e aqueles para o jogo em que ocorrem estratégias não-antecipativas.

Lema 13 Considere o jogo descrito no início deste capítulo. São verdadeiras as seguintes proposições:

1. $\vartheta_{b}^{s}\left(x_{0}\right) \leq \vartheta_{b}\left(x_{0}\right) \leq \vartheta_{\sharp}^{s}$.

2. $\vartheta_{b}^{s}\left(x_{0}\right) \leq \vartheta_{\sharp}\left(x_{0}\right) \leq \vartheta_{\sharp}^{s}$.

Demonstração. Demonstraremos o item 1, uma vez que o item 2 é inteiramente análogo.

Tomemos $x_{0} \in \mathbb{R}^{n}$ e $\varepsilon>0$ arbitrários. Da definição de $\vartheta_{b}$, envolvendo um ínfimo, existe $\bar{\mu} \in \Gamma$ tal que

$$
\vartheta_{b}\left(x_{0}\right)+\varepsilon \geq \mathcal{P}\left(x_{0}, \bar{\mu}[v], v\right) .
$$

Como

$$
\mathcal{P}\left(x_{0}, \bar{\mu}[v], v\right) \geq \inf _{u \in \mathcal{U}} \mathcal{P}\left(x_{0}, u, v\right),
$$

para todo $v \in \mathcal{V}$, vale, em particular que

$$
\mathcal{P}\left(x_{0}, \bar{\mu}[v], v\right) \geq \inf _{u \in \mathcal{U}} \mathcal{P}\left(x_{0}, u, v^{*}\right) \geq \inf _{u \in \mathcal{U}} \sup _{v \in \mathcal{V}} \mathcal{P}\left(x_{0}, u, v\right)=\vartheta_{b}^{s}
$$

Assim, concluímos que $\vartheta_{b}^{s} \leq \vartheta_{b}+\varepsilon$. Como $\varepsilon>0$ é arbitrário, segue a primeira desigualdade.

A segunda desigualdade no item 1 deve-se ao fato de que $\mathcal{U} \subset \Gamma$.

Uma hipótese que cerca a idéia de função de valor no contexto deste trabalho é a continuidade - uma vez que esta é uma hipótese crucial para os resultados envolvendo soluções viscosas. Assim sendo, uma etapa importante na nossa discussão é o estabelecimento da continuidade das funções de valor inferior e superior de um jogo diferencial. 
Veremos adiante que, sob algumas hipóteses, as funções de valor superior e inferior do jogo são uniformemente contínuas e podem atender a um critério de continuidade conhecido como Hölder continuidade.

Definição 50 (Hölder continuidade) Sejam $\Omega_{1} \subset \mathbb{R}^{n}, \Omega_{2} \subset \mathbb{R}^{m}$ e $f: \Omega_{1} \longrightarrow \Omega_{2}$ uma função. Diremos que $f$ é Hölder contínua, com constante positiva $C$ e expoente $\alpha$, se para quaisquer $x, y \in$ $\Omega_{1}$ valer

$$
\|f(x)-f(y)\| \leq C\|x-y\|^{\alpha} .
$$

Claramente, Hölder continuidade implica em continuidade; posto que, da continuidade da norma, $x \longrightarrow y$ implica que $\|f(x)-f(y)\| \longrightarrow 0$.

Um exemplo de uma aplicação Hölder contínua é a função $x \longmapsto \sqrt{x}$, definida sobre $[0,1]$, com constante $C=1$ e expoente $\alpha=0,5$. A figura 6.1 exibe as o gráfico de $(x, y) \longmapsto\left|x^{\frac{1}{2}}-y^{\frac{1}{2}}\right|$ em cinza claro e o gráfico de $(x, y) \longmapsto|x-y|^{\frac{1}{2}}$ em cinza escuro.

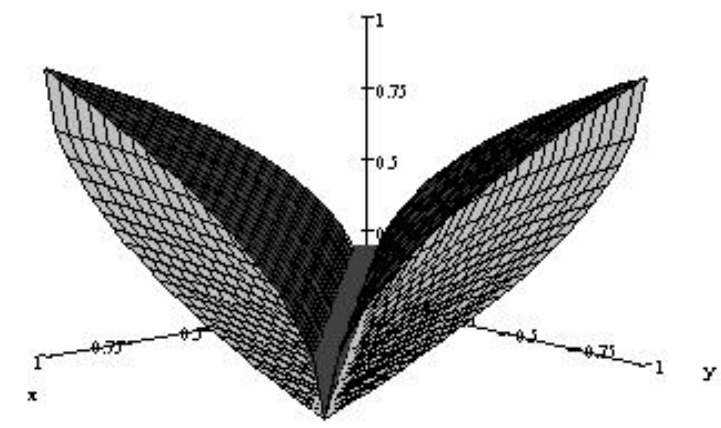

Figura 6.1: $(x, y) \longmapsto\left|x^{\frac{1}{2}}-y^{\frac{1}{2}}\right|$ em cinza claro e $(x, y) \longmapsto|x-y|^{\frac{1}{2}}$ em cinza escuro.

Vejamos agora o resultado de nosso interesse que encerra esta seção.

Proposição 15 Considere 6.1 e suponha que $U$ e $V$ são espaços métricos compactos e que são válidas as estimativas 6.2-6.5. Suponha ainda que

$$
\mathcal{P}\left(x_{0}, u, v\right)=\int_{0}^{T} l(x(t), u(t), v(t)) e^{-t} d t,
$$

onde $l: \mathbb{R}^{n} \times U \times V \longrightarrow \mathbb{R}$ é tal que:

1. $l: \mathbb{R}^{n} \times U \times V \longrightarrow \mathbb{R}$ é continua

2. Existe um módulo de continuidade $m_{l}$ tal que

$$
|l(x, u, v)-l(y, u, v)| \leq m_{l}(\|x-y\|)
$$


para todo $x, y \in \mathbb{R}^{n}, u \in \mathcal{U}$ e $v \in \mathcal{V}$.

3. Existe uma constante $M>0$ tal que

$$
|l(x, u, v)| \leq M
$$

para todo $x, y \in \mathbb{R}^{n}, u \in \mathcal{U}$ e $v \in \mathcal{V}$.

Então $\vartheta_{b}$ e $\vartheta_{\sharp}$ são limitados e uniformemente contínuos em $\mathbb{R}^{n}$. Mais ainda, se for $m_{l}(r)=L_{l} r$, com $L_{l} \neq 1$, então $\vartheta_{b}$ e $\vartheta_{\sharp}$ são Hölder contínuos.

Demonstração. Vamos expor a demonstração para o caso do valor inferior $\vartheta_{b}$, posto que o procedimento para $\vartheta_{\sharp}$ é inteiramente análogo.

$$
\begin{aligned}
& \left|\vartheta_{b}\left(x_{0}\right)\right|=\left|\inf _{u \in \Gamma} \sup _{v \in \mathcal{V}} \int_{0}^{T} l\left(x_{0}(t), u(t), v(t)\right) e^{-t} d t\right| \\
& \leq M \int_{0}^{T}\left|e^{-t}\right| d x \\
& =M\left(1-\frac{1}{e^{T}}\right)<M,
\end{aligned}
$$

o que implica que o valor inferior do jogo é limitado por $M$. Para o caso de um funcional de pagamento do tipo horizonte temporal infinito, podemos obter uma estimativa para

$$
\left|\vartheta_{b}\left(x_{0}\right)\right|=\left|\inf _{u \in \Gamma} \sup _{v \in \mathcal{V}} \int_{0}^{\infty} l\left(x_{0}(t), u(t), v(t)\right) e^{-t} d t\right|
$$

da mesma maneira, considerando que, neste caso,

$$
\left|\vartheta_{b}\left(x_{0}\right)\right| \leq M\left(1-\lim _{T \rightarrow \infty} \frac{1}{e^{T}}\right)=M
$$

Vejamos agora a continuidade uniforme. Para tanto, fixemos dois pontos arbitrários, $x_{0}$ e $y_{0}$, em $\mathbb{R}^{n}$ e, a partir da definição de $\vartheta_{b}$ envolvendo ínfimos e supremos, obtemos um elemento $\bar{u} \in \Gamma$ tal que

$$
\vartheta_{b}\left(y_{0}\right) \geq \sup _{v \in \mathcal{V}} \mathcal{P}\left(y_{0}, \bar{u}(v), v\right)-\frac{\varepsilon}{2}
$$

e um elemento $\bar{v} \in \Delta$ tal que

$$
\vartheta_{b}\left(x_{0}\right) \leq \mathcal{P}\left(x_{0}, \bar{u}(\bar{v}), \bar{v}\right)+\frac{\varepsilon}{2}
$$

Assim, temos que

$$
\vartheta_{b}\left(x_{0}\right)-\vartheta_{b}\left(y_{0}\right) \leq \int_{0}^{\infty} e^{-t}\left|l\left(x_{x_{0}}, \bar{u}(\bar{v}), \bar{v}\right)-l\left(x_{y_{0}}, \bar{u}(\bar{v}), \bar{v}\right)\right| d t+\varepsilon .
$$


Das condições impostas sobre $l$ e sobre $m_{l}$, segue imediatamente que

$$
\begin{gathered}
\vartheta_{b}\left(x_{0}\right)-\vartheta_{b}\left(y_{0}\right) \leq \int_{0}^{T} e^{-t} m_{l}\left(\left\|x_{x_{0}}-x_{y_{0}}\right\|\right) d t+\varepsilon \\
\leq \int_{0}^{T} e^{-t} m_{l}\left(e^{-L T}\left\|x_{0}-y_{0}\right\|\right) d t+\varepsilon \\
\leq m_{l}\left(e^{-L T}\left\|x_{0}-y_{0}\right\|\right) \int_{0}^{T} e^{-t} d t
\end{gathered}
$$

de onde concluímos que

$$
\| \vartheta_{b}\left(x_{0}\right)-\vartheta_{b}\left(y_{0} \| \longrightarrow \infty\right.
$$

uma vez que $\left\|x_{0}-y_{0}\right\| \rightarrow 0$. Da arbitrariedade de $x_{0}$ e de $y_{0}$ segue a continuidade uniforme. Novamente, o argumento pode ser replicado para o caso em que o limite superior de integração $T$ é substituído por $\infty$.

Para a Hölder continuidade, o limite superior de integração deve ser $0<T<\infty$. Se for $L_{l} \neq 1$, temos dois casos. Vejamos inicialmente o que ocorre quando $L-1<0$.

$$
\begin{gathered}
\vartheta_{b}\left(x_{0}\right)-\vartheta_{b}\left(y_{0}\right) \leq m_{l}\left(e^{L_{l} T}\left\|x_{0}-y_{0}\right\|\right) \int_{0}^{T} e^{-t} d t+\varepsilon \\
\leq L_{l}\left\|x_{0}-y_{0}\right\| \int_{0}^{T} e^{(L-1) t} d t+\varepsilon \\
\quad=L_{l}\left\|x_{0}-y_{0}\right\| \frac{e^{(L-1) T}-1}{L-1}+\varepsilon .
\end{gathered}
$$

Fazendo então $T$ tender a infinito e $\varepsilon$ tender a zero, obtém-se o resultado com a constante positiva

$$
\frac{-L_{l}}{L-1}
$$

e o expoente $\alpha=1$.

No caso em que $L-1>0$ tomamos a seguinte estimativa:

$$
\vartheta_{b}\left(x_{0}\right)-\vartheta_{b}\left(y_{0}\right) \leq L_{l}\left\|x_{0}-y_{0}\right\| \frac{e^{(L-1) T}-1}{L-1}+2 M e^{-T}+\varepsilon .
$$

Se fixarmos um valor de

$$
T=\frac{1}{L} \ln \frac{2 M}{L_{l}\left\|x_{0}-y_{0}\right\|}>0
$$

então,

$$
L_{l}\left\|x_{0}-y_{0}\right\| \frac{e^{\frac{L-1}{L} \ln \frac{2 M}{L_{l}\left\|x_{0}-y_{0}\right\|}}-1}{L-1}+2 M e^{-\frac{1}{L} \ln \frac{2 M}{L_{l}\left\|x_{0}-y_{0}\right\|}}+\varepsilon
$$




$$
\begin{aligned}
& =\frac{L_{l}\left\|x_{0}-y_{0}\right\| L_{l}\left\|x_{0}-y_{0}\right\|^{\frac{1}{l}-1}}{2 M(L-1)}+L_{l}\left\|x_{0}-y_{0}\right\|^{\frac{1}{L}}+\varepsilon \\
& =\left(\frac{L_{l}^{2}}{2 M(L-1)}+L_{l}\right)\left\|x_{0}-y_{0}\right\|^{\frac{1}{L}} .
\end{aligned}
$$

Assim, vemos que

$$
\left|\vartheta_{b}\left(x_{0}\right)-\vartheta_{b}\left(y_{0}\right)\right| \leq K\left\|x_{0}-y_{0}\right\|^{\alpha}
$$

onde

$$
K=\frac{L_{l}^{2}}{2 M(L-1)}+L_{l}
$$

$\mathrm{e}$

$$
\alpha=\frac{1}{L} .
$$

Como $K>0$, obtemos a Hölder continuidade.

Este resultado encerra esta seção. Na verdade, nosso interesse na próxima seção será demonstrar dois resultados cruciais: $(i)$ o princípio da programação dinâmica para as funções de valor que dependem de dois controles ou parâmetros que será utilizado na demonstração do fato (ii) de que as funções de valor, inferiores e superiores, são soluções viscosas das equações. HJI, respectivamente, inferiores e superiores.

\subsection{Funções de valor e soluções viscosas da equação HJI}

Nesta seção será discutido o conjunto de condições que devemos estabelecer sobre um jogo a fim de que este possua uma função de valor no sentido de Friedman.

Para tanto, exporemos dois resultados cruciais, a saber: $(i)$ o princípio da programação dinâmica (PPD) para sistemas controláveis a dois parâmetros e (ii) o teorema que verifica que a função de valor inferior [superior] é uma solução viscosa da equação de HJI inferior [superior] associada ao jogo.

Neste sentido, vamos reunir os elementos que determinam nosso jogo diferencial a partir de agora. Em primeiro lugar temos a dinâmica

$$
\dot{x}(t)=f(x(t), u(t), v(t)), \quad t>0 \quad e \quad x(0)=x_{0},
$$

onde $f: \mathbb{R}^{n} \times U \times V \longrightarrow \mathbb{R}^{n}$, com $U$ e $V$ espaços métricos compactos, e $f$ satisfaz uma condição de Lipschitz dada pela expressão

$$
(f(x, a, b)-f(y, a, b)) \cdot(x-y) \leq L\|x-y\|^{2},
$$

com $L>0$.

A função de pagamento do jogo é dada por 


$$
\mathcal{P}\left(x_{0}, u, v\right)=\int_{0}^{\infty} l\left(x_{x_{0}}(t), u(t), v(t)\right) e^{-t} d t
$$

onde $l$ satisfaz às seguintes condições:

\section{Condição 2 1. $l: \mathbb{R}^{n} \times U \times V \longrightarrow \mathbb{R}$ é continua.}

2. Existe um módulo de continuidade $m_{l}$ tal que

$$
|l(x, u, v)-l(y, u, v)| \leq m_{l}(\|x-y\|)
$$

para todo $x, y \in \mathbb{R}^{n}, u \in \mathcal{U}$ e $v \in \mathcal{V}$.

3. Existe uma constante $M>0$ tal que

$$
|l(x, u, v)| \leq M
$$

para todo $x, y \in \mathbb{R}^{n}, u \in \mathcal{U}$ e $v \in \mathcal{V}$.

Desta maneira, os valores inferiores e superiores serão dados por:

$$
\begin{gathered}
\vartheta_{b}\left(x_{0}\right)=\inf _{\mu \in \Gamma} \sup _{v \in \mathcal{V}} \int_{0}^{\infty} l\left(x_{x_{o}}(t), \mu[v](t), v(t)\right) e^{-t} d t \\
\vartheta_{\sharp}\left(x_{0}\right)=\sup _{\nu \in \Delta} \inf _{u \in \mathcal{U}} \int_{0}^{\infty} l\left(x_{x_{o}}(t), u(t), \nu[u](t)\right) e^{-t} d t .
\end{gathered}
$$

Vejamos estes resultados.

Teorema 14 [Princípio da Programação Dinâmica] Suponha 6.1 e 6.2-6.5 e a condição 2 sobre $l$ válida. Então, para todo $x_{0} \in \mathbb{R}^{n}$ e para todo $t>0$ :

$$
\vartheta_{b}\left(x_{0}\right)=\inf _{\mu \in \Gamma} \sup _{v \in \mathcal{V}}\left\{\int_{0}^{t} l\left(x_{x_{o}}(s), \mu[v](s), v(s)\right) e^{-s} d s+\vartheta_{b}\left(x_{x_{0}}(t), \mu[v](t), v(t)\right) e^{-t}\right\}
$$

$e$

$$
\vartheta_{\sharp}\left(x_{0}\right)=\sup _{\nu \in \Delta} \inf _{u \in \mathcal{U}}\left\{\int_{0}^{t} l\left(x_{x_{o}}(s), u(s), \nu[u](s)\right) e^{-s} d s+\vartheta_{\sharp}\left(x_{x_{0}}(t), u(t), \nu[u](t)\right) e^{-t}\right\}
$$

Demonstração. Vamos oferecer a demonstração para 6.11, uma vez que 6.12 poderá ser obtida de forma completamente análoga.

Denote o lado direito de 6.11 por $w\left(x_{0}\right)$, ou seja, 


$$
w\left(x_{0}\right)=\inf _{\mu \in \Gamma} \sup _{v \in \mathcal{V}}\left\{\int_{0}^{t} l\left(x_{x_{o}}(s), \mu[v](s), v(s)\right) e^{-s} d s+\vartheta_{b}\left(x_{x_{0}}(t), \mu[v](t), v(t)\right) e^{-t}\right\}
$$

e fixemos $\varepsilon>0$ arbitrário.

Da definição de $\vartheta_{b}$ existe $\mu_{z} \in \Gamma$ tal que

$$
\vartheta_{b}(z) \geq \sup _{v \in \mathcal{V}} \mathcal{P}\left(z, \mu_{z}[v], v\right)-\varepsilon
$$

Mostraremos inicialmente que $\vartheta_{b}\left(x_{0}\right) \leq w\left(x_{0}\right)$. Novamente, pelo fato da definição de $w$ envolver um ínfimo, temos que existe $\bar{\mu} \in \Gamma$ tal que

$$
w\left(x_{0}\right) \geq \sup _{v \in \mathcal{V}}\left\{\int_{0}^{t} l\left(x_{x_{0}}(s), \bar{\mu}[v](s), v(s)\right) e^{-s} d s+\vartheta_{b}\left(x_{x_{0}}(t, \bar{\mu}[v](t), v(t))\right) e^{-t}\right\}-\varepsilon
$$

Vamos definir agora um controle $\delta \in \Gamma$ dado por

$$
\delta[v](s) \doteq\left\{\begin{array}{cl}
\bar{\mu}[v](s) & s \leq t, \\
\mu_{z}[v](s+t)(s-t) & s>t,
\end{array}\right. \text { onde }
$$

$\mathrm{z} \doteq x_{x_{0}}(t, \bar{\mu}[v](t), v(t))$.

Observamos que, da propriedade de semi-grupo das soluções, temos que

$$
x_{x_{0}}(s+t, \delta[v], v)=x_{z}\left(s, \mu_{z}[v](s+t), v(s+t)\right) .
$$

Afirmamos que, através da mudança de variáveis

$$
\tau=s+t
$$

temos

$$
\mathcal{P}\left(z, \mu_{z}[v](s+t), v(s+t)\right)=\int_{t}^{\infty} l\left(x_{x_{0}}(\tau), \delta[v](\tau), v(\tau)\right) e^{t-\tau} d \tau .
$$

Por um lado, temos que

$$
\begin{array}{r}
\sup _{v \in \mathcal{V}}\left\{\int_{0}^{t} l\left(x_{x_{0}}, \bar{\mu}[v], v\right) e^{-s} d s+\vartheta_{b}\left(x_{x_{0}}(t, \bar{v}[v], v)\right) e^{-t}\right\} \geq \\
\left.\geq \sup _{v \in \mathcal{V}}\left\{\int_{0}^{t} l\left(x_{x_{0}}, \bar{\mu}[v], v\right) e^{-s} d s+\mathcal{P}\left(z, \mu_{z}[v](s+t), v s+t\right)\right)-\varepsilon\right\},
\end{array}
$$


o que implica que

$$
w\left(x_{x_{0}} \geq \sup _{v \in \mathcal{V}}\left\{\int_{0}^{\infty} l\left(x_{x_{0}}, \delta[v], v\right) e^{-s} d s\right\}-2 e .\right.
$$

Por outro lado, é evidente que, da definição de $\vartheta_{b}$, temos

$$
\vartheta_{b}\left(x_{x_{0}}\right) \leq \sup _{v \in \mathcal{V}}\left\{\int_{0}^{\infty} l\left(x_{x_{0}}, \delta[v], v\right) e^{-s} d s\right\}
$$

Assim, concluímos que $w\left(x_{x_{0}}\right) \geq \vartheta_{b}\left(x_{x_{0}}\right)-2 \varepsilon$ e, como $\varepsilon$ é arbitrário, segue que

$$
w\left(x_{0}\right) \geq \vartheta_{b}\left(x_{0}\right)
$$

Vejamos agora que $w\left(x_{0}\right) \leq \vartheta_{b}\left(x_{0}\right)$. Tome $v_{1} \in \mathcal{V}$ tal que

$$
w\left(x_{0}\right) \leq \int_{0}^{t} l\left(x_{x_{0}}, \mu_{x_{0}}\left[v_{1}\right], v_{1}\right) e^{-s} d s+\vartheta_{b}\left(x_{0}\left(t, \mu_{x_{0}}\left[v_{1}\right], v_{1}\right)\right) e^{-t}+\varepsilon,
$$

com $\mu_{x_{0}}$ definido como anteriormente (em 6.14). Tal $v_{1}$ existe, novamente, pelo fato da definição de $w$ envolver a noção de ínfimo e supremo.

Agora, vamos definir uma aplicação $v \longmapsto \tilde{v}$ tal que, para cada $v \in \mathcal{V}$ temos

$$
\tilde{v}(s) \doteq\left\{\begin{array}{cc}
v_{1}(s) & s \leq t \\
v(s-t) & s>t
\end{array}\right.
$$

Definimos ainda $\tilde{\mu} \in \Gamma$ por $\tilde{\mu}[v](s)=\mu_{x_{0}}[\tilde{v}](s+t), z=x_{x_{0}}\left(t, \mu\left[v_{1}\right], v_{1}\right)$ e $v_{2} \in \mathcal{V}$ tal que

$$
\vartheta_{b}(z) \leq \mathcal{P}\left(z, \tilde{\mu}\left[v_{2}\right], v_{2}\right)+\varepsilon
$$

Como veremos adiante nesta demonstração,

$$
w\left(x_{0}\right) \leq \mathcal{P}\left(x_{0}, \mu_{x_{0}}\left[\tilde{v}_{2}\right], \tilde{v}_{2}\right)+\varepsilon,
$$

o que implica que

$$
w\left(x_{0}\right) \leq \vartheta_{b}\left(x_{0}\right)+3 \varepsilon .
$$

Novamente, como $\varepsilon$ é arbitrário, segue 


$$
w\left(x_{0}\right) \leq \vartheta_{b}\left(x_{0}\right)
$$

Para concluirmos nossa demonstração será necessário provarmos 6.16. Para tanto, basta observar que

$$
x_{x_{0}}\left(\tau, \mu_{x_{0}}\left[\tilde{v}_{2}\right], \tilde{v}_{2}\right)=\left\{\begin{array}{cc}
x_{x_{0}}\left(\tau, \mu_{x_{0}}\left[v_{1}\right], v_{1}\right) & \tau \leq t, \\
x_{x_{0}}\left(\tau-t, \tilde{\mu}\left[v_{2}\right], v_{2}\right) & \tau>t,
\end{array}\right.
$$

o que, pela mudança de coordenadas $\tau=s+t$ implica em

$$
\mathcal{P}\left(z, \tilde{\mu}\left[v_{2}\right], v_{2}\right)=\int_{0}^{\infty} l\left(x_{x_{0}}(\tau), \mu_{x_{0}}\left[\tilde{v}_{2}\right](\tau), \tilde{v}_{2}(\tau)\right) e^{t-\tau} d \tau
$$

Desta forma, concluímos que

$$
w\left(x_{0}\right) \leq \int_{0}^{\infty} l\left(x_{x_{0}}(\tau), \mu_{x_{0}}\left[\tilde{v}_{2}\right](\tau), \tilde{v}_{2}(\tau)\right) e^{-\tau} d \tau+2 \varepsilon
$$

o que culmina em 6.16 e, assim, conclui a demonstração.

Estabelecido este resultado, nossa tarefa será a demonstração de que as funções de valor de um jogo diferencial conforme definido nesta seção satisfazem uma equação diferencial parcial de primeira ordem, não linear, a chamada equação de Hamilton-Jacobi-Isaacs. Para isto vamos introduzir os Hamiltonianos associados ao jogo diferencial.

Definição 51 Considere o jogo diferencial descrito no início deste capítulo. A aplicação $H_{b}$ : $\mathbb{R}^{n} \times \mathbb{R}^{n} \longrightarrow \mathbb{R}$ dada por

$$
H_{b}(x, p) \doteq \min _{u \in \mathcal{U}} \max _{v \in \mathcal{V}}\{-f(x, u, v) \cdot p-l(x, a, b)\}
$$

é chamada de Hamiltoniano inferior associado ao jogo diferencial.

Já a aplicação $H_{\sharp}: \mathbb{R}^{n} \times \mathbb{R}^{n} \longrightarrow \mathbb{R}$ dada por

$$
H_{\sharp}(x, p) \doteq \max _{v \in \mathcal{V}} \min _{u \in \mathcal{U}}\{-f(x, u, v) \cdot p-l(x, a, b)\}
$$

é chamada de Hamiltoniano superior associado ao jogo diferencial.

Será com base nestes elementos que definiremos nossas EDP's, a partir das quais a questão da existência da função de valor de Friedman será abordada.

Para iniciarmos esta discussão, apresentaremos formalmente nossas equações.

Definição 52 A equação diferencial parcial

$$
\vartheta+H_{b}(x, D \vartheta)=0 \quad e m \quad \mathbb{R}^{n}
$$


será chamada de equação de Hamilton-Jacobi-Isaacs inferior. Já a equação

$$
\vartheta+H_{\sharp}(x, D \vartheta)=0 \quad \text { em } \quad \mathbb{R}^{n}
$$

será chamada de equação de Hamilton-Jacobi-Isaacs superior.

Assim definidas, estas equações podem ser escritas na linguagem utilizada em nosso capítulo anterior, ou seja,

$$
F_{b}(x, \vartheta, D \vartheta)=0
$$

para o caso do Hamiltoniano inferior e

$$
F_{\sharp}(x, \vartheta, D \vartheta)=0
$$

para o caso do Hamiltoniano superior.

O resultado mais importante deste capítulo - e, quiçá, deste trabalho - estabelecerá que, no contexto do jogo aqui descrito e sob a condição 2, então a função de valor inferior é uma solução viscosa da HJI inferior e a função de valor superior é uma solução viscosa da HJI superior.

Entretanto, como uma das hipóteses mais utilizadas em nosso capítulo anterior sobre a função $F$ era sua continuidade, dedicaremos o próximo lema ao estabelecimento da continuidade das equações de HJI superior e inferior.

\section{Lema 14}

$$
F_{b}(x, \vartheta, p) \in \mathcal{C}\left(\mathbb{R}^{n} \times \mathbb{R} \times \mathbb{R}^{n}\right)
$$

$e$

$$
F_{\sharp}(x, \vartheta, p) \in \mathcal{C}\left(\mathbb{R}^{n} \times \mathbb{R} \times \mathbb{R}^{n}\right) .
$$

Demonstração. Ambas são claramente contínuas em $\vartheta$ e em $p$. Para vermos que a continuidade em $x$ também se verifica, basta aplicarmos a proposição 14 .

Vejamos agora mais um lema, este com vistas à próxima demonstração.

Lema 15 Considere a dinâmica dada pela equação 6.1 sob a condição dada por 6.2 e a condição 2 sobre a função de pagamento. Sejam então $x_{0} \in \mathbb{R}^{n}$ e a aplicação $\phi \in \mathcal{C}^{1}\left(\mathbb{R}^{n}\right)$ tais que

$$
\phi\left(x_{0}\right)+H\left(x_{0}, D \phi\left(x_{0}\right)\right)=\xi>0 .
$$

Então, existem $\mu^{*} \in \Gamma$ e $t^{*}>0$ tais que para todo $v \in \mathcal{V}$ tem-se

$$
\int_{0}^{t^{*}}\left\{l\left(x_{x_{0}}, \mu^{*}(v), v\right)+f\left(x_{x_{0}}, \mu^{*}(v), v\right) \cdot D \phi\left(x_{x_{0}}\right)-\phi\left(x_{x_{0}}\right)\right\} e^{-s} d s \leq-\frac{\xi t}{4} .
$$


Demonstração. Vamos definir inicialmente uma aplicação $h: \mathbb{R}^{n} \times \mathcal{U} \times \mathcal{V} \longrightarrow \mathbb{R}$ da seguinte maneira:

$$
h(x, u, v) \doteq \phi(x)-l(x, u, v)-f(x, u, v) \cdot D \phi(x) .
$$

Por envolver operações elementares de funções contínuas em $x, h$ é também contínua em $x$. Pela mesma razão, $h$ é contínua em $u$ e $v$. Como, no entanto, $\mathcal{U}$ e $\mathcal{V}$ são conjuntos compactos, $h$ é uniformemente contínua nestas variáveis.

Ainda sobre $h$, como estimamos que $\left\|x_{x_{0}}(t, \cdot, \cdot)-x_{0}\right\| \leq M_{x_{0}} t$, e de sua continuidade em $x$, temos que para todo $\varepsilon>0$ existe $t^{*}>0$ tal que

$$
\left|h\left(y_{x_{0}}(t), \cdot, \cdot\right)-h\left(x_{0}, \cdot, \cdot\right)\right|<\varepsilon
$$

para todo $0<t<t^{*}$. Tomemos agora o máximo de $h$ com relação a $u$ e, em seguida, o mínimo de $h$ com relação a $v$. Temos:

$$
\begin{array}{r}
\min _{v} \max _{u} h\left(x_{x_{0}}, u, v\right)=\phi\left(x_{x_{0}}\right)+\min _{v} \max _{u}\left(-l\left(x_{x_{0}}, u, v\right)-f\left(x_{x_{0}}, u, v\right) \cdot D \phi\left(x_{x_{0}}\right)\right) \\
=\phi\left(x_{x_{0}}\right)+H\left(x_{x_{0}}, D \phi\left(x_{x_{0}}\right)\right)=\xi .
\end{array}
$$

Dado $v^{*} \in \mathcal{V}$, seja $u^{*} \in \mathcal{U}$ tal que

$$
h\left(x_{x_{0}}, u^{*}, v^{*}\right)=\max _{u} h\left(x_{x_{0}}, u, v^{*}\right) .
$$

É claro que

$$
h\left(x_{x_{0}}, u^{*}, v^{*}\right) \geq \min _{v} \max _{u} h\left(x_{x_{0}}, u, v\right) .
$$

Afirmamos que, pela continuidade uniforme de $h$ em $v$, existe uma vizinhança de $v^{*}$ na qual $h\left(x_{x_{0}}, u^{*}, v^{*}\right) \geq \xi$. Em particular,

$$
h\left(x_{x_{0}}, u^{*}, \zeta\right) \geq \frac{3 \xi}{4}
$$

para todo $\zeta$ em alguma vizinhança de $v^{*}$, digamos, $\mathcal{B}_{r\left(v^{*}\right)}\left(v^{*}\right)$.

Vamos agora tomar uma cobertura aberta de $\mathcal{V}$. Considere a coleção de conjuntos abertos dada por

$$
B=\left\{\mathcal{B}_{r(v)}(v) \mid v \in \mathcal{V}\right\}
$$

assim, se $v$ é um ponto de $\mathcal{V}$, então $v$ é o centro de uma destas bolas abertas e, portanto, está contido na união dos elementos da coleção. Desta maneira, $B$ é uma cobertura por abertos do compacto $\mathcal{V}$. Logo,

$$
\mathcal{V} \subset \bigcup_{i=1}^{n} \mathcal{B}_{r_{i}}\left(v_{i}\right)
$$

onde $r_{i}=r\left(b_{i}\right)$. 
Defina $u_{i}=u\left(v_{i}\right)$ tal que

$$
h\left(x_{x_{0}}, u_{i}, v_{i}\right)=\max _{u} h\left(x_{x_{0}}, u, v_{i}\right) .
$$

Da discussão anterior sobre $h$ é evidente que

$$
h\left(x_{x_{0}}, u_{i}, \zeta\right) \geq \frac{3 \xi}{4}, \quad \forall \zeta \in \mathcal{B}_{r_{i}}\left(v_{i}\right)
$$

Vamos definir agora uma aplicação $\Psi: \mathcal{V} \longrightarrow \mathcal{U}$. Uma vez que cada $v$ pertence a alguma das bolas abertas $\mathcal{B}_{r_{i}}$ tomamos

$$
\Psi(v)=u_{k}
$$

se

$$
v \in \mathcal{B}_{r_{k}}\left(b_{k}\right) \backslash \bigcup_{i=1}^{k-1} \mathcal{B}_{r_{i}}\left(b_{i}\right)
$$

Assim,

$$
\mu[v](s) \doteq \Psi(v(s))
$$

e obtemos que

$$
h\left(x_{x_{0}}(s), \mu[v](s), v(s)\right) \geq \frac{\xi}{2},
$$

para todo $v \in \mathcal{V}$ e para todo $s$ suficientemente pequeno, digamos, $s<t^{*}$.

Nosso último passo será multiplicar ambos os membros da desigualdade em 6.23 pela quantidade negativa $-e^{-t}$ e integrar entre 0 e $s<t^{*}$. Assim obtemos que

$$
\int_{0}^{s}\left\{l\left(x_{x_{0}}, \mu[v], v\right)+f\left(x_{x_{0}}, \mu[v], v\right) \cdot D \phi\left(x_{x_{0}}\right)-\phi\left(x_{x_{0}}\right)\right\} e^{-t} d t \leq \int_{0}^{s}-e^{-t} \frac{\xi}{2} d t
$$

e, uma vez que

$$
\int_{0}^{s}-e^{t} \frac{\xi}{2} d t \leq-\frac{\xi}{2}\left(1-e^{-s}\right) \leq-\frac{\xi}{4} s
$$

obtemos a desigualdade desejada.

Corolário 4 Considere a dinâmica dada pela equação 6.1 sob a condição dada por 6.2 e a condição em 2 sobre a função de pagamento. Sejam então $x_{0} \in \mathbb{R}^{n}$ e a aplicação $\phi \in \mathcal{C}^{1}\left(\mathbb{R}^{n}\right)$ tais que

$$
\phi\left(x_{0}\right)+H\left(x_{0}, D \phi\left(x_{0}\right)\right)=\xi>0 .
$$

Então, existem $\mu^{*} \in \Gamma$ e $t^{*}>0$ tais que para todo $v \in \mathcal{V}$ tem-se

$$
\int_{0}^{t^{*}}\left\{l\left(x_{x_{0}}, \mu^{*}(v), v\right)\right\} e^{-s} d s \leq-\frac{\zeta t}{4} .
$$


Demonstração. Basta notarmos que do lema anterior (15) temos

$$
\int_{0}^{t^{*}}\left\{l\left(x_{x_{0}}, \mu^{*}(v), v\right)+f\left(x_{x_{0}}, \mu^{*}(v), v\right) \cdot D \phi\left(x_{x_{0}}\right)-\phi\left(x_{x_{0}}\right)\right\} e^{-s} d s \leq-\frac{\xi t}{4}
$$

que pode ser re-escrita como

$$
\int_{0}^{t^{*}} l\left(x_{x_{0}}, \mu^{*}(v), v\right) e^{-s} d s \leq-\int_{0}^{t^{*}} f\left(x_{x_{0}}, \mu^{*}(v), v\right) \cdot D \phi\left(x_{x_{0}}\right)-\phi\left(x_{x_{0}}\right) e^{-s} d s-\frac{\xi t}{4} .
$$

Tomando o segundo membro desta desigualdade vemos que

$$
-\int_{0}^{t^{*}} f\left(x_{x_{0}}, \mu^{*}(v), v\right) \cdot D \phi\left(x_{x_{0}}\right)-\phi\left(x_{x_{0}}\right) e^{-s} d s-\frac{\xi t}{4} \leq-t^{*}\left(M+\frac{\xi}{4}\right)
$$

onde

$$
M=\sup \left|f\left(x_{x_{0}}, \mu^{*}(v), v\right) \cdot D \phi\left(x_{x_{0}}\right)-\phi\left(x_{x_{0}}\right) e^{-s}\right| .
$$

Assim, basta colocarmos

$$
\frac{\zeta}{4}=M+\frac{\xi}{4}>0
$$

e a demonstração está concluída.

Passemos agora ao resultado de maior relevância do capítulo.

Teorema 15 Considere o jogo descrito no início deste capítulo e suponha válidas as condições 6.1, 6.2 sobre a dinâmica do jogo e 2 sobre a função de pagamento. Então, a função de valor inferior do jogo é solução viscosa da HJI inferior

$$
\vartheta+H_{b}(x, D \vartheta)=0 \quad \text { em } \quad \mathbb{R}^{n}
$$

e a função de valor superior $\vartheta_{\sharp}$ é solução viscosa da HJI superior

$$
\vartheta+H_{\sharp}(x, D \vartheta)=0 \quad \text { em } \quad \mathbb{R}^{n} .
$$

Demonstração. Mostraremos o resultado para o caso do valor inferior, $\vartheta_{b}$, uma vez que o caso restante é inteiramente análogo. A demonstração tem dois passos. Inicialmente verificamos que $\vartheta_{b}$ é sub-solução viscosa da equação de HJI, utilizando o lema anterior e o princípio da programação dinâmica. Em seguida, veremos que $\vartheta_{b}$ é ainda uma super-solução viscosa desta mesma equação, de maneira análoga.

Suponhamos por absurdo que $\vartheta_{b}$ não seja sub-solução viscosa de

$$
\vartheta+H_{b}(x, D \vartheta)=0 .
$$


Então existe uma função $\phi \in \mathcal{C}^{1}\left(\mathbb{R}^{n}\right)$ tal que $x^{*}$ é ponto de máximo de $\vartheta_{b}-\phi$ e

$$
\phi\left(x^{*}\right)+H\left(x^{*}, D \phi\left(x^{*}\right)\right)=\xi>0 .
$$

Vemos então que, para valores pequenos de $t$, eventualmente $t<t^{*}$,

$$
\vartheta_{b}\left(x^{*}\right)-\phi\left(x^{*}\right) \geq \vartheta_{b}\left(x_{x^{*}}(t)\right)-\phi\left(x_{x^{*}}(t)\right) .
$$

Para $t<t^{*}$, ainda podemos obter

$$
\vartheta_{b}\left(x^{*}\right)-\phi\left(x^{*}\right) \geq e^{-t} \vartheta_{b}\left(x_{x^{*}}(t)\right)-e^{-t} \phi\left(x_{x^{*}}(t)\right) .
$$

Assim, temos

$$
e^{-t} \phi\left(x_{x^{*}}(t)\right)-\phi\left(x^{*}\right) \geq e^{-t} \vartheta_{b}\left(x_{x^{*}}(t)\right)-\vartheta_{b}\left(x^{*}\right) .
$$

Utilizando o corolário 4, temos que

$$
\int_{0}^{t^{*}} l\left(x_{x^{*}}(s), \mu^{*}[v](s), v(s)\right) e^{-s} d s \leq-t^{*} \frac{\zeta}{4}
$$

de onde segue que

$$
\int_{0}^{t^{*}} l\left(x_{x^{*}}(s), \mu^{*}[v](s), v(s)\right) e^{-s} d s+e^{-t^{*}} \phi\left(x_{x^{*}}\left(t^{*}\right)\right)-\phi\left(x^{*}\right) \leq-t^{*} \frac{\zeta}{4},
$$

uma vez que $t^{*}$ é arbitrariamente pequeno. Reunindo as estimativas acima vemos que para todo $v \in \mathcal{V}$, existe $\mu^{*} \in \Gamma$ tal que

$$
\int_{0}^{t^{*}} l\left(x_{x^{*}}(s), \mu^{*}[v](s), v(s)\right) e^{-s} d s+e^{-t^{*}} \vartheta_{b}\left(x_{x^{*}}\left(t^{*}\right)\right)-\vartheta_{b}\left(x^{*}\right) \leq-t^{*} \frac{\zeta}{4} .
$$

Em particular,

$$
\inf _{\mu \in \Gamma} \sup _{v \in \mathcal{V}}\left\{\int_{0}^{t^{*}} l\left(x_{x^{*}}(s), \mu^{*}[v](s), v(s)\right) e^{-s} d s+e^{-t^{*}} \vartheta_{b}\left(x_{x^{*}}\left(t^{*}\right)\right)\right\}-\vartheta_{b}\left(x^{*}\right) \leq-t^{*} \frac{\zeta}{4}<0 .
$$

Logo, obtemos que

$$
\vartheta_{b}\left(x^{*}\right)>\inf _{\mu \in \Gamma} \sup _{v \in \mathcal{V}}\left\{\int_{0}^{t^{*}} l\left(x_{x^{*}}(s), \mu^{*}[v](s), v(s)\right) e^{-s} d s+e^{-t^{*}} \vartheta_{b}\left(x_{x^{*}}\left(t^{*}\right)\right)\right\},
$$

o que é uma contradição em vistas do princípio da programação dinâmica, verificado no teorema 14 . Assim, $\vartheta_{b}$ é sub-solução viscosa da HJI associada.

Vejamos agora que $\vartheta_{b}$ é super-solução da mesma equação de HJI e, portanto, é solução viscosa. Para tanto considere uma função $\psi \in \mathcal{C}^{1}$ e um ponto $x_{*}$, mínimo local de $\vartheta_{b}-\psi$, i.e., 


$$
\vartheta_{b}\left(x_{*}\right)-\psi\left(x_{*}\right) \leq \vartheta_{b}\left(x_{x_{*}}(t)\right)-\psi\left(x_{x_{*}}(t)\right)
$$

para valores pequenos de $t$ digamos, $0<t<t^{*}$. Suponhamos por absurdo que

$$
\psi\left(x_{*}\right)+H\left(x, D \psi\left(x_{*}\right)\right)=-\xi<0 .
$$

Da definição de $H$ segue que

$$
\psi\left(x_{*}\right)+\min _{u} \max _{v}\left\{-f\left(x_{*}, u, v\right) \cdot D \psi\left(x_{*}\right)-l\left(x_{*}, u, v\right)\right\}=-\xi<0 .
$$

Logo, para todo $u_{*} \in \mathcal{U}$ dado, existe $v_{*} \in \mathcal{V}$ tal que

$$
\psi\left(x_{*}\right)-f\left(x_{*}, u_{*}, v_{*}\right) \cdot D \psi\left(x_{*}\right)-l\left(x_{*}, u_{*}, v_{*}\right) \leq-\xi .
$$

Considerando $0<t<t^{*}$, ainda temos

$$
\psi\left(x_{x_{*}}(t)\right)-f\left(x_{x_{*}}(t), u_{*}(t), v_{*}(t)\right) \cdot D \psi\left(x_{x_{*}}(t)\right)-l\left(x_{x_{*}}(t), u_{*}(t), v_{*}(t)\right) \leq-\frac{\xi}{2} .
$$

Multiplicando ambos os lados da desigualdade por $e^{-s}$ e integrando entre 0 e $t^{*}$ obtemos que

$-\int_{0}^{t^{*}} l\left(x_{x_{*}}(t), u_{*}(t), v_{*}(t)\right) e^{-t} d t \leq-\int_{0}^{t^{*}}\left(\psi\left(x_{x_{*}}(t)\right)-f\left(x_{x_{*}}(t), u_{*}(t), v_{*}(t)\right) \cdots D \psi\left(x_{x_{*}}(t)\right)\right) e^{-t} d t \leq-t^{*} \frac{\xi}{4}$,

de onde temos, por razão análoga à mencionada anteriormente, que

$$
-\int_{0}^{t^{*}} l\left(x_{x_{*}}(t), u_{*}(t), v_{*}(t)\right) e^{-t} d t \leq-t^{*} \frac{\zeta}{4}<0 .
$$

Da última desigualdade segue que

$$
\psi\left(x_{*}\right)-e^{t^{*}} \psi\left(x_{x_{*}}\right)-\int_{0}^{t^{*}} l\left(x_{x_{*}}(t), u_{*}(t), v_{*}(t)\right) e^{-t} d t \leq-t^{*} \frac{\zeta}{4}<0 .
$$

Então, de 6.26, segue que

$$
e^{t^{*}} \vartheta_{b}\left(x_{x_{*}}\right)+\int_{0}^{t^{*}} l\left(x_{x_{*}}(t), u_{*}(t), v_{*}(t)\right) e^{-t} d t \geq \vartheta_{b}\left(x_{*}\right)+t^{*} \frac{\zeta}{4}<0
$$

Assim, obtemos que

$$
\inf _{\mu \in \Gamma} \sup _{v \in \mathcal{V}}\left\{\int_{0}^{t^{*}} l\left(x_{x_{*}}(t), \mu[v](t), v(t)\right) e^{-t} d t+\vartheta_{b}\left(x_{x_{*}}\left(t^{*}\right) \mu[v]\left(t^{*}\right), v\left(t^{*}\right)\right) e^{-t^{*}}\right\}>\vartheta_{b}\left(x_{*}\right),
$$

o que é uma contradição, novamente, em vistas do princípio da programação dinâmica. 
Desta maneira, vemos que a função de valor inferior [superior] é uma solução viscosa da equação de Hamilton-Jacobi-Isaacs inferior [superior].

Até este momento sabemos que um jogo diferencial possui sempre uma função de valor inferior e uma função de valor superior, cada uma associada ao Hamiltoniano, inferior ou superior, do jogo.

Suponha agora que ambos estes Hamiltonianos sejam iguais, i.e.,

$$
H_{b}=H_{\sharp},
$$

ou , mais precisamente, que

$$
\min _{u \in \mathcal{U}} \max _{v \in \mathcal{V}}\{-f(x, u, v) \cdot p-l(x, u, v)\}=\max _{v \in \mathcal{V}} \min _{u \in \mathcal{U}}\{-f(x, u, v) \cdot p-l(x, u, v)\} .
$$

Então, $\vartheta_{b}$ e $\vartheta_{\sharp}$ serão duas soluções viscosas da mesma equação HJI. Se, no entanto, $H=H_{b}=$ $H_{\sharp}$ satisfizer ainda à condição 1 , sabemos que esta equação HJI possui uma única solução, pelo corolário 3 do teorema da comparação visto no capítulo 5. Assim, obtemos o

Teorema 16 Considere o jogo diferencial cuja dinâmica é regida por 6.1, sob as condições expostas em 6.2-6.5. Suponha ainda que a função de pagamento do jogo seja dada por

$$
\mathcal{P}\left(x_{0}, u, u\right)=\int_{0}^{T} l(x(s), u(s), v(s)) e^{-s} d s,
$$

onde l satisfaz a condição 2. Se, além disso,

$$
-f(x, u, v) \cdot p-l(x, u, v)
$$

for côncava em $v$ e convexa em u, Então

$$
\bar{H}=H_{b}=H_{\sharp}
$$

e o jogo diferencial tem pelo menos uma função de valor. Mais ainda, se $\bar{H}$ satisfizer a condição 1, então esta solução é única.

Demonstração. Aplicamos primeiramente o teorema do MinMax de Ky Fan (teorema 8). Em seguida, aplicamos o corolário 3 do teorema da comparação e o resultado é imediato.

Assim, concluímos nossa discussão acerca da teoria dos jogos diferenciais em sua conexão com a teoria das equações diferenciais parciais.

\subsection{Comentários finais}

O modelo de jogo que estudamos neste capítulo diz respeito ao caso de dois jogadores, somazero, determinístico. Uma pequena modificação nos argumentos apresentados permite a extensão dos resultados aqui discutidos para o caso estocástico. Ver, por exemplo, [10], [27] e [51].

Ainda para o caso de dois jogadores, soma-zero, estocástico, um interessante exemplo é jogo sugerido por Peres, Schramm, Sheffield e Wilson em [55]. Neste caso, os autores estudam dois 
jogadores que decidem a ordem de jogada de cada um a partir do resultado do lançamento de uma moeda. O principal resultado do trabalho consiste em verificar que funções Lipschitzianas a valores reais, definidas em um subconjunto arbitrário $Y$ de um espaço métrico $X$ - que deve satisfazer um conjunto adicional de hipóteses - possui uma extensão absolutamente minimal para $X$. Isto é discutido levando-se em consideração que a função de valor que surge no contexto deste jogo é uma solução viscosa da equação

$$
\Delta_{\infty} u=0
$$

ou seja, uma solução do infinito-Laplaciano, dado por

$$
\Delta_{\infty}=|\nabla u|^{-2} \sum_{i, j} u_{x_{i}} u_{x_{i} x_{j}} u_{x_{j}}
$$

No que diz respeito a jogos estocásticos envolvendo $N>2$ jogadores, mencionamos a princípio o trabalho de Bensoussan e Frehse [11]. Neste texto os autores discutem uma série de resultados de regularidade envolvendo sistemas elípticos não lineares e abordam jogos estocásticos como uma aplicação desta teoria, sem, entretanto, mencionar a teoria de viscosidade.

Por fim, exemplos de jogos estocásticos envolvendo $N>2$ jogadores e a teoria das soluções viscosas podem ser encontrados no trabalho de Lasry e Lions [15], que estuda a formulação conhecida como mean field games. 


\section{Apêndice A}

\section{O Ponto Fixo de Kakutani}

Neste apêndice vamos expor uma demonstração do teorema do ponto fixo de Kakutani, como uma generalização para o contexto das correspondências - ou aplicações multivaloradas - do teorema do ponto fixo de Brouwer. Vamos iniciar nossa discussão com algumas definições e exemplos.

\section{A.1 Alguns comentários preliminares}

Definição 53 Considere um conjunto de $n+1$ pontos, $\Delta=\left\{v_{0}, v_{1}, \ldots, v_{n}\right\}$ tal que $\left\{v_{i}-v_{0} \mid i=1, \ldots, n\right\}$ seja um conjunto linearmente independente. Então, o menor conjunto convexo que contém $\Delta$ será chamado n-simplex. Cada $v_{i}$ será um vértice do simplex.

Considere a figura A.1. Nela vemos três exemplos de simplices. O primeiro deles é um 2-simplex, o segundo um 7-simplex e o último um 1-simplex.
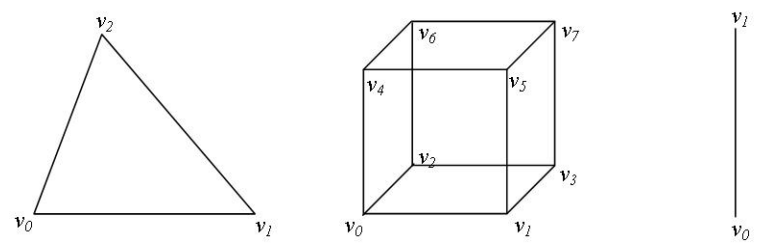

Figura A.1: Exemplos de simplex.

Por outro lado, a condição de que os vetores $v_{i}-v_{0}$ sejam linearmente independentes significa que, para que tenhamos um $n$-simplex, os vértices não podem pertencer a um hiperplano de dimensão menor do que $n$. Observe a figura A.2.

Nesta figura, não é possível encontrarmos um hiperplano $H$ que contenha os quatro vértices. Já em A.3 isto é possível. O resultado é que um dos vértices se torna em um elemento do interior do simplex, e não obtemos um objeto 3-dimensional. Vejamos a figura A.3

Considere agora os vetores da chamada base canônica do $\mathbb{R}^{n}$, ou seja, $\left\{e_{i} \mid i=1, \ldots n\right\}$ onde cada $e_{i}$ possui zeros em todas as suas entradas a menos da $i$-ésima

$$
\begin{aligned}
& e_{1}=(1,0,0, \ldots, 0) \\
& e_{2}=(0,1,0, \ldots, 0)
\end{aligned}
$$




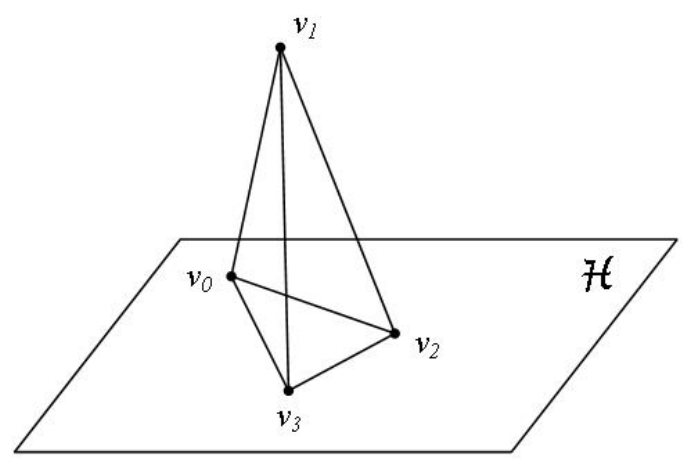

Figura A.2: Tetraedro.

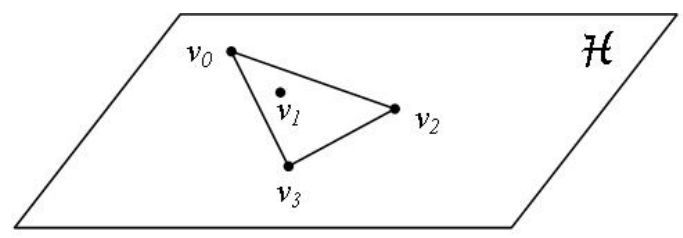

Figura A.3: Conjunto de pontos colineares.

$$
\begin{array}{r}
e_{3}=(0,0,1, \ldots, 0) \\
\vdots \\
e_{n}=(0,0,0, \ldots, 1) .
\end{array}
$$

Definição 54 (Simplex padrão) Um n-simplex cujos vértices são os $n+1$ vetores da base canônica de $\mathbb{R}^{n+1}$ é chamado de padrão ou regular.

Um exemplo de simplex padrão, o caso 2-dimensional em $\mathbb{R}^{3}$, é exibido na figura A.4.

Vamos considerar agora uma aplicação $\phi$ definida sobre os vértices de um $n$-simplex $\mathcal{S}$ padrão tal que $\phi\left(e_{i}\right)=y_{i}$ para todo $i=1, \ldots, n$. Claramente, $\phi$ pode ser estendida a

$$
\Phi: \mathcal{S} \longrightarrow \mathcal{S}
$$

de forma linear. Vejamos o procedimento.

Seja $x \in \mathcal{S}$. Então,

$$
x=\sum_{i=1}^{n} \lambda_{i} e_{i} .
$$

Ponha

$$
\Phi(x)=\sum_{i=1}^{n} \lambda_{i} \phi\left(e_{i}\right) .
$$

É claro que $\Phi$ é linear. 


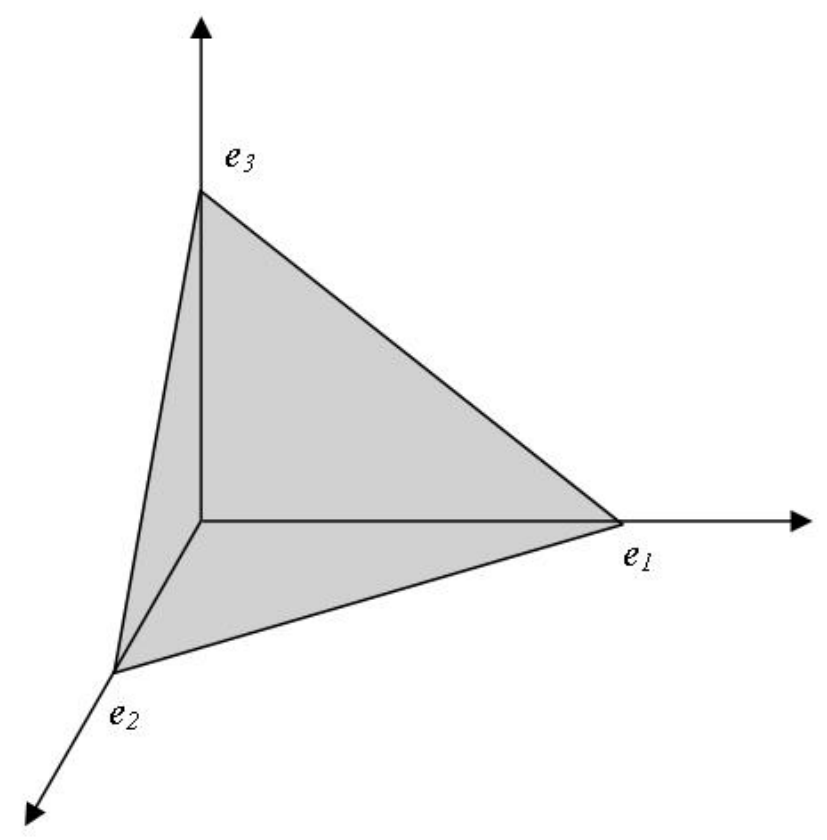

Figura A.4: Simplex padrão em dimensão 2.

Vamos discutir agora a noção de divisão baricêntrica de um simplex.

Definição 55 Seja $\mathcal{S}$ um n-simplex. Então, se adicionarmos um vértice ao centro de $\mathcal{S}$ e outro ao ponto médio de cada um de seus lados, e, em seguida, unirmos cada novo vértice àquele no centro de $\mathcal{S}$ obtemos uma divisão baricêntrica do simplex $\mathcal{S}$

Vemos na figura A.5 um exemplo de divisão baricêntrica do triângulo.

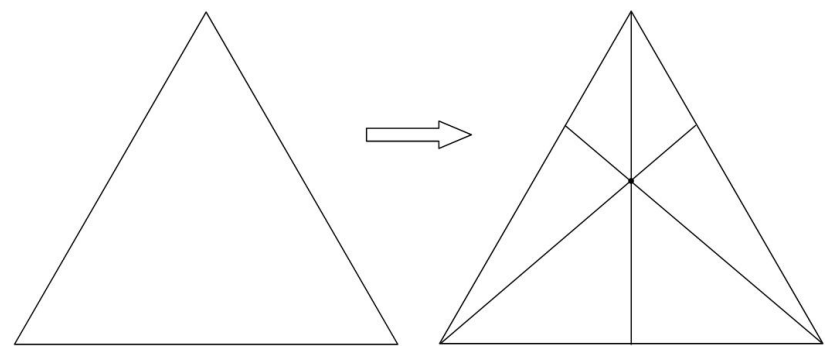

Figura A.5: Exemplo de divisão baricêntrica do triângulo.

É claro que podemos tomar a segunda divisão baricêntrica do triângulo exibido na figura A.5. Bastaria para tanto que em cada 2-sub-complex da primeira divisão, procedêssemos a uma nova divisão. Vejamos o aspecto da segunda divisão baricêntrica do triângulo na figura A.6

De maneira indutiva, podemos proceder a $n$-ésima divisão baricêntrica de um simplex para qualquer número $n \in \mathbb{N}$. É intuitivo que quando $n \longrightarrow \infty$ a área de cada sub-simplex tende a zero e, assim, deve tender a zero também o raio de uma bola aberta nele contido. Esta noção geométrica é de crucial importância no estabelecimento do teorema de Kakutani. 


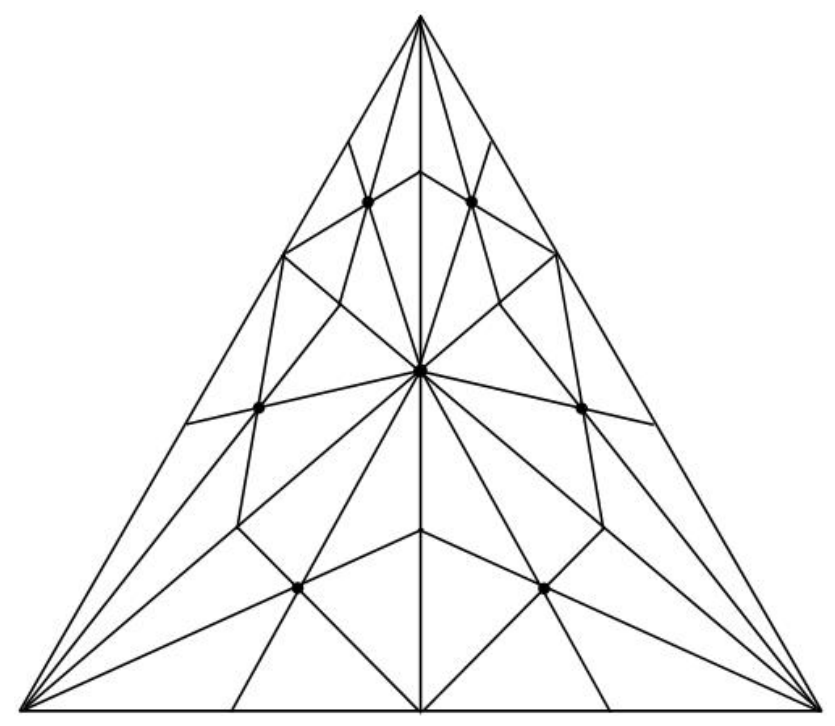

Figura A.6: Segunda divisão baricêntrica do triângulo.

O ponto fixo de Kakutani é uma generalização do teorema de Brouwer. A diferença característica entre eles é que, ao passo em que Brouwer aborda funções, Kakutani endereça correspondências, ou, aplicações multivaloradas.

Definição 56 Seja $X$ um conjunto não vazio. Uma aplicação $\Psi: X \longrightarrow 2^{X}$ é chamada uma correspondência.

Exemplo 11 1. Denote a esfera em $\mathbb{R}^{2}$ por $S^{1}$ e seja $f: S^{1} \longrightarrow 2^{S^{1}}$ a aplicação que a cada ponto $\theta$ associa o arco $0-\theta$. Então, $f(0)=0$ é um único ponto, ao passo em que $f(\pi)$ é o hemisfério norte de $S^{1}$ e $f(2 \pi)$ é o próprio $S^{1}$.

2. Seja $g: \mathbb{N} \backslash\{1\} \longrightarrow \mathbb{N}$ a aplicação que, dado um número natural, associa o conjunto de seus divisores diferentes de 1 . É claro que g é multivalorada.
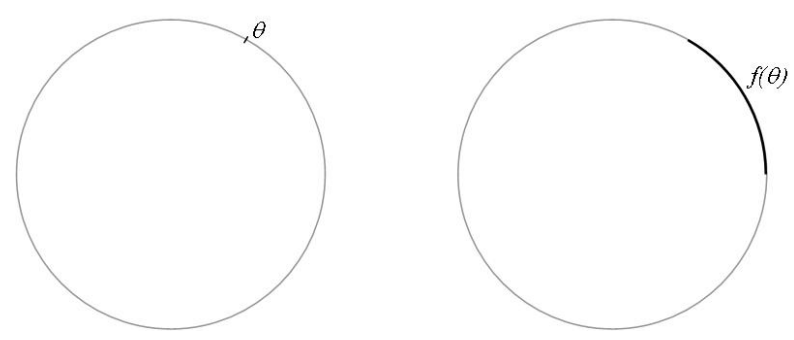

Figura A.7: $f$ associa a $\theta$ o arco $0-\theta$.

Vamos apresentar na próxima seção o teorema do ponto fixo de Brouwer e a generalização devida a Kakutani, assim como sua demonstração. 


\section{A.2 Enunciado e demonstração}

A esta altura o leitor deve entender que um ponto fixo de uma função $f$ é um elemento $\bar{x}$ do domínio de $f$ tal que $f(\bar{x})=\bar{x}$. No entanto, em se tratando de uma correspondência, esta noção pode não ser a mais clara possível. Vamos então a uma definição:

Definição 57 Seja $\Psi: X \longrightarrow 2^{X}$ uma correspondência. Então, $\bar{x} \in X$ é dito um ponto fixo para $\Psi$ se $\bar{x} \in \Psi(\bar{x})$.

Foi dito aqui que Kakutani generaliza o teorema do ponto fixo devido a Brouwer. Vejamos como este pode ser enunciado.

Teorema 17 (do ponto fixo de Brouwer) Seja $X$ um sub-conjunto compacto de $\mathbb{R}^{n}$ e $f: X \longrightarrow$ $X$ uma função contínua. Então, $f$ tem pelo menos um ponto fixo.

A demonstração deste resultado não será exposta aqui. ${ }^{1}$

Vejamos agora um primeiro enunciado do teorema do ponto fixo de Kakutani para $n$-simplices. Ao provarmos o teorema para o caso dos $n$-simplices, estaremos prontos para estender sua afirmação a qualquer variedade diferenciável convexa compacta. Vejamos:

Teorema 18 (do ponto fixo de Kakutani) Sejam $\mathcal{S}$ um $r$-simplex e $2^{\mathcal{S}}$ o conjunto de suas partes. Considere ainda uma correspondência

$$
\Psi: \mathcal{S} \longrightarrow 2^{\mathcal{S}}
$$

tal que $\Psi(x)$ é um conjunto convexo para todo $x \in \mathcal{S}$ e $\Psi$ é semi-contínua superiormente ${ }^{2}$. Então, $\Psi$ possui, ao menos, um ponto fixo.

Demonstração. Seja $\mathcal{S}^{(1)}$ a primeira divisão baricêntrica de $\mathcal{S}$. Tome os vértices de $\mathcal{S}^{(1)}$, a saber, $x_{1}^{(1)}, x_{2}^{(1)}, \ldots, x_{k}^{(1)}$, e aplique $\Psi$ a cada um deles. Tome, em cada $\Psi\left(x_{i}^{(1)}\right)$ um ponto $y_{i}^{(1)}$ e defina a aplicação

$$
\psi_{(1)}\left(x_{i}^{(1)}\right)=y_{i}^{(1)}
$$

Estendendo linearmente $\psi_{(1)}$ a $\mathcal{S}^{(1)}$ para uma função $\psi_{(1)}^{*}$, vemos que esta última é contínua do compacto $\mathcal{S}^{(1)}$ em $\mathcal{S}^{(1)}$. Logo, pelo teorema do ponto fixo de Brouwer, $\psi_{(1)}^{*}$ tem um ponto fixo que denotaremos $x_{(1)}^{*}$

Seja agora $\mathcal{S}^{(2)}$ a segunda divisão baricêntrica de $\mathcal{S}$ e definamos $\psi_{(2)}\left(x_{i}^{(2)}\right)=y_{i}^{(2)}$ como feito anteriormente. Então, sua extensão $\psi_{(2)}^{*}$ terá um ponto fixo $x_{(2)}^{*}$. Procedendo desta maneira indefinidamente, obteremos uma seqüência de pontos $\left(x_{(n)}^{*}\right)_{n \in \mathbb{N}}$, os pontos fixos das funções $\psi_{(n)}^{*}$.

\footnotetext{
${ }^{1} \mathrm{O}$ leitor pode encontrá-la em diversos contextos. Para uma discussão deste teorema no contexto introdutório da análise real, sugerimos [39]. Já para uma discussão do resultado como uma conseqüência do grupo fundamental de $S^{1}$, ver [40] e [31]. Em [31], este resultado é ainda discutido sob a luz dos grupos de homologia singular da esfera.

${ }^{2}$ Ver a definição 31
} 
Como esta seqüência está contida em $\mathcal{S}$, um compacto, ela possui uma subseqüência convergente. Vamos assumir, por apelo da notação, que esta seqüência é ela mesma convergente, i.e.,

$$
x_{(n)}^{*} \longrightarrow x^{*}, \quad n \longrightarrow \infty
$$

Afirmamos que $x^{*}$ será o ponto fixo de $\Psi$.

Para vermos isto, considere o sub-simplex $\Delta^{n}$ que contém o ponto $x_{(n)}^{*}$ e sejam $\delta_{1}^{(n)}, \delta_{2}^{(n)}, \ldots, \delta_{r}^{(n)}$ seus vértices. Então note que

$$
x_{(n)}^{*}=\sum_{i=1}^{r} \lambda_{i}^{(n)} \delta_{i}^{(n)},
$$

$\operatorname{com} \lambda_{i}^{(n)} \geq 0$ e $\sum_{i=1}^{r} \lambda_{i}^{(n)}=1 \mathrm{e}$

$$
\delta_{i}^{(n)} \longrightarrow x^{*}
$$

quando $n \longrightarrow \infty$.

A primeira observação decorre do fato de que um ponto de um simplex pode sempre ser escrito como a combinação linear ${ }^{3}$ convexa de seus vértices. Já a segunda, segue da observação anterior de que o volume de cada sub-simplex da $n$-ésima divisão baricêntrica tende a zero quando $n$ cresce.

Pelos mesmos motivos do argumento anterior, $y_{i}^{(n)}$ e $\lambda_{i}^{(n)}$ possuem sub-seqüências convergentes que denotaremos - novamente em benefício da simplicidade da notação - por $y_{i}^{(n)}$ e $\lambda_{i}^{(n)}$ tais que

$$
y_{i}^{(n)} \longrightarrow y_{i}^{*}
$$

$\mathrm{e}$

$$
\lambda_{i}^{(n)} \longrightarrow \lambda_{i}^{*}
$$

É fácil ver que

$$
y_{i}^{(n)}=\psi_{(n)}\left(x_{i}^{(n)}\right) \in \Psi\left(x_{i}^{(n)}\right) .
$$

Mais ainda,

$$
x_{(n)}^{*}=\psi\left(x_{(n)}^{*}\right)=\sum_{i=1}^{r} \lambda_{i}^{(n)} y_{i}^{(n)} .
$$

Tomando os limites, vemos que

$$
x^{*}=\sum_{i=1}^{r} \lambda_{i}^{*} y_{i}^{*} .
$$

\footnotetext{
${ }^{3}$ Combinação linear é entendida aqui como uma soma finita.
} 
Assim verifica-se que

$$
\delta_{i}^{(n)} \longrightarrow x^{*}
$$

$\mathrm{e}$

$$
y_{i}^{(n)} \in \Psi\left(\delta_{i}^{(n)}\right)
$$

Da hipótese de semi-continuidade sobre $\Psi$, vemos que

$$
y_{i}^{*} \in \Psi\left(x^{*}\right)
$$

para todo $i=1, \ldots, r$. Da equação A.1 e da convexidade de $\Psi\left(x^{*}\right)$ segue que

$$
x^{*}=\sum_{i=1}^{r} \lambda_{i}^{*} y_{i}^{*} \in \Psi\left(x^{*}\right),
$$

e, assim, segue o resultado do teorema.

A demonstração acima se encarrega do resultado para o contexto dos simplices. Observe entretanto que

Proposição 16 Seja $M$ uma variedade diferenciável compacta e convexa e considere uma correspondência $\Psi: M \longrightarrow 2^{M}$ semi-contínua superiormente cuja imagem em cada ponto de $M$ é um subconjunto convexo não vazio. Então existe $x^{*} \in M$ tal que $x^{*} \in \Psi\left(x^{*}\right)$.

Demonstração. Observe que qualquer variedade $M$ compacta pode ser circunscrita a um simplex $S$, que se torna uma triangulação de $M$. Para uma discussão deste importante resultado, remetemos o leitor a [31]. Como $M$ e qualquer uma triangulação sua são homeomorfas e, mais ainda, o ponto fixo é uma propriedade invariante topológica segue da existência do ponto fixo em $S$ o resultado desejado.

Desta maneira expusemos a demonstração do resultado chave para o estabelecimento do equilíbrio de Nash, conceito solução fundamental à teoria dos jogos.

Finalizamos esta seção exibindo alguns exemplos que evidenciam a importância das hipóteses envolvidas no teorema de Kakutani.

Exemplo 12 Sejam $I=[a, b]$ e $\psi_{1}: I \longrightarrow I$

$$
\psi_{1}(x) \doteq \begin{cases}\{b\} & x \in\left[a, \frac{a+b}{2}\right] \\ \{a\} & x \in\left(\frac{a+b}{2}, b\right]\end{cases}
$$

$\psi_{1}$ não tem pontos fixos. I é um conjunto compacto e convexo. Mais ainda, $\psi_{1}(x)$ é um conjunto convexo para todo $x \in I$. Entretanto, considere a seqüência de pontos $\left(\xi_{n}\right)_{n \in \mathbb{N}}=\left(x_{n}, \psi_{1}\left(x_{n}\right)\right)_{n \in \mathbb{N}}$ onde $x_{n} \longrightarrow \frac{a+b}{2}$ e $x_{n}>\frac{a+b}{2}$ para todo $n \in \mathbb{N}$. Então, $\xi_{n} \longrightarrow\left(\frac{a+b}{b} ; a\right)$ que não pertence ao gráfico de $\psi_{1}$. Logo, a aplicação $\psi_{1}$ não possui um gráfico fechado e, portanto, não é semi-contínua superiormente. 
Exemplo 13 Considere entretanto

$$
\psi_{2}(x) \doteq\left\{\begin{array}{cc}
\{b\} & x \in\left[a, \frac{a+b}{2}\right) \\
\{a, b\} & x=\frac{a+b}{2} \\
\{a\} & x \in\left(\frac{a+b}{2}, b\right] .
\end{array}\right.
$$

$\psi_{2}$ também não possui pontos fixos. Note que, neste caso, $\psi_{2}\left(\frac{a+b}{2}\right)$ não é convexo, o que viola outra hipótese do teorema.

Exemplo 14 Se tomarmos entretanto

$$
\psi_{3}(x) \doteq\left\{\begin{array}{cc}
\{b\} & x \in\left[a, \frac{a+b}{2}\right) \\
{[a, b]} & x=\frac{a+b}{2} \\
\{a\} & x \in\left(\frac{a+b}{2}, b\right]
\end{array}\right.
$$

vemos que $\psi_{3}\left(\frac{a+b}{2}\right)=[a, b] \ni \frac{a+b}{2}$. Ou seja, $\psi_{3}$ possui um ponto fixo. Note que do exemplo anterior para este, a única mudança foi tornar a correspondência convexa em todo ponto do domínio.

Vemos os gráficos das aplicações mencionadas nos exemplos acima na figura A.8.
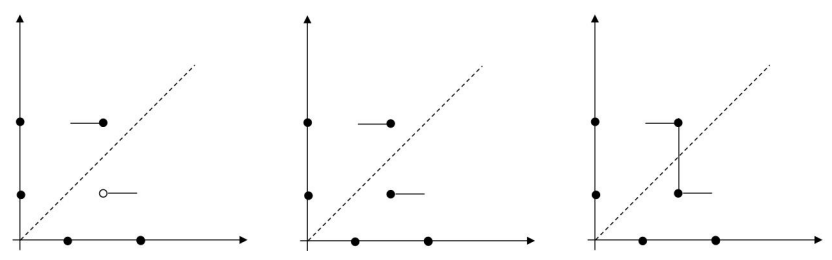

Figura A.8: Gráficos, $\psi_{1}, \psi_{2}$ e $\psi_{3}$, respectivamente.

Assim, finalizamos este apêndice dedicado ao teorema de Kakutani acerca dos pontos fixos de correspondências em dimensão finita. 


\section{Referências Bibliográficas}

[1] J. Green A. Mas-Colell, R. Whinston, Microeconomic theory, primeira ed., Oxford University Press, Nova-Iorque, 1995.

[2] M. Bardi, M.G. Crandall, L.C. Evans, H.M. Soner, and P.E. Souganidis, Viscosity solutions and applications, primeira ed., Lecture Notes in Mathematics, Vol. 1660, Springer, Heidelberg, 1997.

[3] R. Bellman, Dynamic programming, primeira ed., Princeton University Press, Princeton, 1957.

[4] D. Bertsekas, Dynamic programming and optimal control, vol. 1, terceira ed., Athena Scientific, Belmont, 2005.

[5] O.N. Bondereva, Some applications of linear programming methods to the theory of cooperative game, Problemy Kibernetiki 10 (1963), 119-139.

[6] S. Hart e A. Mas-Colell, Potential, value and consistency, Econometrica 57 (1989), 589-614.

[7] D. Fudenberg e D.K. Levine, Reputation and equilibrium selection in games with a patient player, Econometrica 57 (1989), 759-778.

[8] K.J. Arrow e G. Debreu, Existence of an equilibrium for a competitive economy, Econometrica 22 (1954), 265-290.

[9] G. Debreu e H. Scarf, A limit theorem on the core of an economy, Int. Econ. Review 4 (1963), $235-246$.

[10] M.Bardi e I. Capuzzo-Dolcetta, Optimal control and viscosity solutions of Hamilton-JacobiBellman equations, segunda ed., Birkhäuser, Boston, 2008.

[11] A. Bensoussan e J. Frehse, Regularity results for nonlinear elliptic systems and applications, primeira ed., Springer, Berlim, 2002.

[12] H. J. N. Lopes e M. C. Lopes, Uma introdução a soluções de viscosidade para equação de Hamilton-Jacobi, primeira ed., IMPA, Rio de Janeiro, 1997.

[13] M. Kaneko e M.H. Wooders, Cores of partitioning games, Math. Soc. Sci. 3 (1982), 313-327.

[14] R.J. Elliott e N. J. Kalton, The existence of value in differential games, primeira ed., Memoirs of the American Mathematical Society - 126, AMS, Providence, 1972.

[15] J-M. Lasry e P-L. Lions, Mean field games, Japan. J. Math. 2 (2007), 229-260.

[16] M.G. Crandall e P.L. Lions, Viscosity soutions of Hamilton-Jacobi equations, Trans. Amer. Math. Soc. 277 (1983), 1-42. 
[17] _ On existence and uniqueness of solutions of Hamilton-Jacobi equations, Nonlinear Analysis, Theory, Methods and Applications 10 (1986), 353-370.

[18] J.C. Harsanyi e R. Selten, A general theory of equilibrium selection in games, primeira ed., MIT Press, Cambridge, 1988.

[19] D.M. Kreps e R. Wilson, Sequential equilibria, Econometrica 50 (1982), 863-894.

[20] F.J. Anscombe e R.J. Aumann, A definition of subjective probability, Annals of Mathematical Statistics 34 (1963), 199-205.

[21] L.C. Evans, On solving certain nonlinear partial differential equations by accretive operator methods, Israel Journal of Mathematics 36 (1980), 225-247.

[22] _ Partial differential equations, primeira ed., AMS, Providence, 2002.

[23] K. Fan, Minimax theorems, Proceedings of the National Academy of Sciences 39 (1953), 42-47.

[24] A. Friedman, Differential games, primeira ed., John Wiley Sons, Nova Iorque, 1971.

[25] J.I. Glicksberg, A further generalization of Kakutani fixed point theorem, Proceedings of the American Mathematical Society 3 (1952), 170-174.

[26] D.A. Gomes, Calculus of variations and partial differential equations, Preprint, UTL-IST (2009).

[27] _ Viscosity soutions of Hamilton-Jacobi equations, CAMGSD-IST, preprint (2010).

[28] J.Y. Halpern, A nonstandard characterization of sequential equilibrium, perfect equilibrium, and proper equilibrium, International Journal of Game Theory 38 (2009), 37-49.

[29] J.C. Harsanyi, Games with incomplete information played by Bayesian players, Management Sci. 14 (1967-68), 159-182, 320-334, 486-502.

[30] P. Hartman, Ordinary differential equations, segunda ed., Classics in Applied Mathematics, 38, SIAM, Philadelphia, 2002.

[31] A. Hatcher, Algebraic topology, primeira ed., Cambridge University Press, Cambridge, 2002.

[32] R. Isaacs, Differential games: a mathematical theory with applications to warfare and pursuit, control and optimization, primeira ed., John Willey and Sons, Nova Iorque, 1965.

[33] H. Ishii, Perron's method for Hamilton-Jacobi equations, Duke Math. J. 55 (1987), 369-384.

[34] F. John, Partial differential equations, quarta ed., Springer-Verlag, Nova Iorque, 1981.

[35] J. F. Nash Jr., Equilibrium points in n-person games, Proc. Nat. Acad. Sci. 36 (1950), 48-49.

[36] S. Kakutani, A generalization of Brouwer's fixed point theorem, Duke Mathematical Journal 8 (1941), 457-458.

[37] D. Kirk, Optimal control theory - an introduction, primeira ed., Prentice Hall, Engleoowd Cliffs, 1970.

[38] S. Koike, A beginner's guide to the theory of viscosity solutions, primeira ed., Mathematical Society of Japan, Tóquio, 2004. 
[39] E. L. Lima, Análise real, volume 1, primeira ed., IMPA, Rio de Janeiro, 1996.

[40] _ Grupo fundamental e espaços de recobrimento, terceira ed., IMPA, Rio de Janeiro, 2006.

[41] W.F. Lucas, An overview of the mathematical theory of the games, Management Sci. 18 (1972), $3-19$.

[42] P.L. Lions e L.C. Evans M.G. Crandall, Some properties of viscosity soutions of HamiltonJacobi equations, Trans. Amer. Math. Soc. 282 (1984), 487-502.

[43] R. Myerson, Refinement of the Nash equilibrium concept, International Journal of Game Theory 7 (1978), 73-80.

[44] R. B. Myerson, Game theory: analysis of conflict, primeira ed., Harvard University Press, Cambridge, 1997.

[45] G. Owen, Game theory, segunda ed., Academic Press, Nova Iorque, 1982.

[46] M. Quincampoix, Differential games, In: Encyclopedia of Complexity and Systems Sciences. Eds. Marilda Sotomayor. Springer, 2009, pp. 1948-1956.

[47] P.J. Reny, On the existence of pure and mixed strategy Nash equilibria in discontinuous games, Econometrica 67 (1999), 1029-1056.

[48] L.J. Savage, The foundations of statistics, primeira ed., Wiley, Nova Iorque, 1954.

[49] L.S. Shapley, A value for n-person games, In: Contributions to the theory of games II. Eds. H.W. Kuhn e A.W. Tucker. Princeton University Press, 1953, pp. 303-317.

[50] _ On balanced sets and cores, Naval research logistics quarterly 14 (1967), 453-460.

[51] P.E. Souganidis, Viscosity soutions of Hamilton-Jacobi equations and differential games, UT Austin, preprint (2004).

[52] J. v Neumann, Zur theorie der gessellshaftspiele, Mathematische Annalen 100 (1928), 295-320.

[53] J. v Neumann e O. Morgenstern, Theory of games and economic behavior, primeira ed., Princeton University Press, Princeton, 1947.

[54] E. van Damme, Stability and perfection of Nash equilibria, segunda ed., Springer, Berlin, 1996.

[55] S. Sheffield D.B. Wilson Y. Peres, O. Schramm, Tug-of-war and the infinity laplacian, Journal of the American Mathematical Society 22 (2009), 167-210. 


\section{Índice Remissivo}

$S^{1}, 130$

Conjunto de informação, 6

Correspondências, 130

Equações diferenciais

características, 63

Euler-Lagrange, 64

Hamilton-Jacobi

Bellman, 1, 54

Isaacs, 62

ordinárias, 1

Equilíbrio

Pareto-eficiente, 21

perfeito, 32

sequencial completo, ou forte, 29

sequencial fraco, 28

de Nash, 15

sequencial, 26

Estratégia

$\delta$-inferior, 48

$\delta$-superior, 47

dominada, 16

mista, 18

não-antecipativa, 109

pura, 18

Forma Bayesiana, 11

Forma extensiva, 4

Forma multi-agente, 13

Forma normal ou estratégica, 8

Forma tipo-agente, 15

Função

côncava, 41

convexa, 41

semi-contínua, 41

uniformemente-módulo-contínua, 86

Função de pagamento, 39

Função de Valor valor $\delta$-inferior, 50

inferior, 114

segundo Friedman, 41

segundo Isaacs, 40, 46

superior, 114

valor $\delta$-superior, 50

Hamiltoniano, 65

inferior, 118

superior, 118

Jogo diferencial

$\delta$-inferior, 50

$\delta$-superior, 49

segundo Friedman, 47, 50

segundo Isaacs, 39

Jogo residual, 17

Lagrangiano, 63

Módulo de continuidade, 85

Princípio da programação dinâmica, 53, 56, 115

Princípio de comparação, 82

Princípio do máximo, 82

Propriedade da intersecção finita, 43

Retro-alimentação, 59

Simplex padrão ou regular, 128

Simplexo, 127

Sub-diferencial, 68

Sub-solução viscosa, 68

Super-diferencial, 68

Super-solução viscosa, 68

Teorema

de Taylor, 55

do ponto fixo de Brouwer, 131

do Minimax, 44

Teorema de comparação, 90 
Teorema de existência de solução viscosa, 104

Transformada de Legendre, 65

Unicidade da solução viscosa, 90 